\title{
HIDROCARBUROS CUTICULARES DE TRIATOMINOS: SU APLICACIÓN COMO MARCADORES TAXONÓMICOS
}

\author{
Gustavo Calderón Fernández \\ Facultad de Ciencias Naturales y Museo \\ Universidad Nacional de La Plata \\ 2008
}


FACULTAD DE CIENCIAS NATURALES Y MUSEO

AÑO 2008

"Hidrocarburos Cuticulares de Triatominos: Su Aplicación Como Marcadores Taxonómicos"

Tesis presentada por el Licenciado Gustavo Mario Calderón Fernández para optar al título de Doctor en Ciencias Naturales

Directora: Dra. Marta Patricia Juárez, Investigador Independiente del Consejo Nacional de Investigaciones Científicas y Tecnológicas, Instituto de Investigaciones Bioquímicas de La Plata

Co-director: Dr. Ricardo Pollero, Profesor Titular de la Facultad de Ciencias Naturales y Museo, Universidad Nacional de La Plata. 
Dedicada a mi madre, Norma. 


\section{Agradecimientos}

A mi familia, a todos mis amigos, a mi novia, que me contuvieron en los momentos difíciles y me dieron fuerza para seguir.

A mi directora, codirector y compañeros de laboratorio que me tuvieron paciencia y me ayudaron siempre en todo lo que pudieron.

Al CONICET que me otorgó la beca que me permitió realizar este docotorado.

Al Servicio Nacional de Chagas de Córdoba y especialmente al Dr. Raúl Stariolo por proveerme de muestras del subcomplejo T. sordida.

A los miembros de la red ECLAT y del CDIA por la provisión de muestras de $T$. dimidiata y del subcomplejo T. sordida. 


\section{INDICE}

Resumen

I. Introducción

I. 1. Enfermedad de Chagas: características generales, epidemiología y distribución. 012

I. 2. Triatominos: características morfológicas y biológicas, clasificación taxonómica. 013

I. 3. Características biológicas del subcomplejo Triatoma sordida. 016

I. 4. Características biológicas de Triatoma dimidiata. 018

I. 5. Hidrocarburos cuticulares en insectos 019

I. 6. Hidrocarburos cuticulares en triatominos 024

I. 7. Análisis cuantitativo y cualitativo de los hidrocarburos. Cromatografía Gaseosa

Capilar (CGC) y CGC acoplada a Espectrometría de Masa (CGC-MS). 029

I. 8. Nuevas técnicas y marcadores de aplicación en taxonomía. 035

I. 9. Aplicación de los hidrocarburos cuticulares en taxonomía de insectos y plantas. 036

I. 10. Métodos de ordenación y clasificación en el estudio de la taxonomía de insectos. 038

\section{Objetivos}

\section{Materiales y Métodos}

III. 1. Insectos.

III. 2. Obtención de los lípidos cuticulares y purificación de la fracción de hidrocarburos.

III. 3. Análisis de los hidrocarburos cuticulares mediante CGC y CGC-MS. 044

III. 4. Análisis taxonómico.

\section{Resultados}

IV. 1. Subcomplejo Triatoma sordida.

IV. 1. 1. Identificación estructural de los hidrocarburos cuticulares mediante CGC-MS 050

IV. 1. 2. Análisis de los hidrocarburos cuticulares mediante CGC, composición en función de las especies y las localidades de colecta.

IV. 1. 3. Análisis taxonómico basado en el patrón de hidrocarburos cuticulares.

IV. 2. Triatoma dimidiata.

IV. 2. 1. Análisis de los hidrocarburos cuticulares mediante CGC, composición en función de las localidades de colecta.

IV. 2. 2. Análisis taxonómico basado en el patrón de hidrocarburos cuticulares. 


\section{Discusión}

V. 1. Estructura de los hidrocarburos cuticulares en el subcomplejo T. sordida.

V. 2. Taxonomía del subcomplejo T. sordida.

V. 3. Análisis de la variabilidad intraespecífica en $T$. dimidiata mediante el patrón de hidrocarburos cuticulares.

V. 4. Conclusiones finales

VI. Referencias bibliográficas.

VII. Abreviaturas 


\section{Resumen}

La subfamilia Triatominae (Hemiptera: Reduvidae), comprende un grupo de insectos hematófagos transmisores de la Enfermedad de Chagas, endemia latinoamericana que afecta cerca de 10 millones de personas, causada por el protozoo flagelado Trypanosoma cruzi. Dadas las dificultades en el desarrollo de una vacuna contra la enfermedad, todos los esfuerzos están volcados al control de los triatominos, como insectos vectores.

Los triatominos colonizan vivienda humana (hábitat doméstico), así como los ambientes próximos a ella (hábitat peridoméstico), con mayor o menor grado de adaptación dependiendo de la especie en consideración. Las especies asociadas al hombre suelen mostrar una amplia distribución geográfica, en parte como resultado de su transporte pasivo en los enseres domésticos durante las migraciones humanas. Se ha agrupado a las más de 130 especies que integran la subfamilia en distintos complejos y subcomplejos en base a similitudes morfológicas y de distribución geográfica, entre otras.

El subcomplejo Triatoma sordida, incluído dentro del complejo T. infestans, está integrado por las especies T. sordida, T. garciabesi, T. guasayana y $T$. patagonica distribuidas diferencialmente en el cono sur de América del Sur (Argentina, Bolivia, Brasil y Paraguay). Se encuentran principalmente en los ambientes silvestres y peridomésticos y tienen una alta tasa de infección con $T$. cruzi. Suelen colonizar la vivienda humana tras la erradicación de T. infestans y T. brasiliensis, por lo que dentro de los programas de control se las considera especies secundarias y potencialmente riesgosas. La variabilidad en diversas características fenotípicas así como la posibilidad de obtener descendencia viable en los cruces experimentales ha llevado a dudar del estatus específico de dos de sus integrantes, $T$. garciabesi y $T$. patagonica. Asimismo, se ha sugerido que $T$. sordida podría estar integrada por dos especies crípticas.

Las tres principales especies vectoras de la enfermedad son $T$. dimidiata, $T$. infestans y Rhodnius prolixus. Dentro del género Triatoma, la especie con mayor capacidad vectorial en América del Norte y Central es $T$. dimidiata. Con una alta tasa de infección con $T$. cruzi, esta especie tiene una alta adaptabilidad a diversas condiciones climáticas y coloniza fácilmente los ambientes peridoméstico y doméstico a partir de sus reservorios silvestres, pudiendo encontrársela incluso en grandes ciudades. De origen centroamericano, actualmente tiene una amplia distribución desde el centro de Mexico, por todos los países de América Central hasta Venezuela, Colombia, Perú y Ecuador en América del Sur. Debido a la gran variabilidad que presenta en sus características morfológicas y etológicas a lo largo de su distribución geográfica, inicialmente fue dividida en subespecies, aunque posteriormente fue reunificada en uno de los estudios más abarcativos hechos sobre taxonomía de triatominos. Sin embargo, 
numerosos estudios posteriores, empleando diversas técnicas fenotípicas y genéticas, muestran una variabilidad difícil de atribuir a la de una única especie.

Los caracteres fenotípicos (citológicos, morfométricos, isoenzimáticos, etc.) y genéticos (obtenidos mediante los métodos de RAPD, rDNA ITS-1 y 2, 18S RNA, entre otros), analizados con diversas técnicas de análisis multivariado, se han empleado exhaustivamente en la taxonomía de varios grupos de animales, plantas, hongos y bacterias, ayudando a dilucidar y en parte a resolver relaciones que la taxonomía clásica no era capaz de definir. El patrón de hidrocarburos cuticulares de insectos se ha utilizado ampliamente como carácter taxonómico en el estudio de diversas especies; entre las de importancia sanitaria se destacan las de los géneros Glossina sp., Simulium sp. y Anopheles sp. En triatominos se conoce el patrón de hidrocarburos de varias especies y complejos, y se ha determinado su estructura en T. infestans, T. mazzottii y R. prolixus.

Dentro del subcomplejo T. sordida, en este trabajo de tesis se pudo discriminar a $T$. garciabesi como especie distinta de T. sordida, en función de las caracteristicas cuali y cuantitativas de su perfil de hidrocarburos; sin embargo T. patagonica no se diferencia como especie, agrupándose como una población de $T$. guasayana. T. sordida y $T$. garciabesi mostraron una relación estrecha entre ambas, siendo $T$. guasayana la especie más diferenciada del subcomplejo. Las poblaciones de $T$. sordida quedaron divididas a su vez en dos grupos netamente diferenciados, que podrían ser considerados como subespecies, en coincidencia con otros estudios.

El análisis de los hidrocarburos de $T$. dimidiata reveló que es necesaria una reconsideración de la taxonomía de la especie. Se obtuvieron tres grupos claramente diferenciados, que se corresponden con la clasificación en subespecies hecha originalmente: T. dimidiata maculipennis para la mayoría de los ejemplares mexicanos, $T$. d. dimidiata para los centroamericanos y $T$. $d$. capitata para los de la mayor parte de los sudamericanos. Adicionalmente, se obtuvo un grupo formado por los insectos de la región mexicana de Yucatán y norte de Guatemala que en coincidencia con otros análisis constituiría otra especie o subespecie. Los insectos colectados en los sistemas de cuevas de Lanquín (Guatemala) y en la región cercana de Augustine (Bélice) mostraron un nivel de diferenciación comparable al de otra especie.

Los resultados obtenidos en esta tesis muestran que el patrón de hidrocarburos es un marcador taxonómico de gran utilidad y sensibilidad, tanto para discernir las relaciones interespecíficas como para evaluar la variabilidad intraespecífica en triatominos, además permite inferir el movimiento de insectos tanto dentro de una región geográfica como entre ambientes. La información aportada, en conjunto con la obtenida mediante otros marcadores 
genéticos y fenéticos contribuiría en el diseño de estrategias específicas de los programas de control de la enfermedad. 


\section{Abstract}

The subfamily Triatominae (Hemiptera: Reduvidae) comprises a group of hematophagous insects, vectors of Chagas disease, an endemic Latin American illness caused by the protozoan flagellate Trypanosoma cruzi, affecting 10 million people. Due to problems in developing a vaccine against the disease, the efforts of control programmes are addressed to control the insect vector.

Triatomines colonize the human dwelling (domestic habitat) as well as the habitat next to the houses (peridomestic habitat); the degree of invasion depends on the species under consideration. Human-related species usually show a large geographic distribution mainly due to pasive transport in people's luggage during migrations. The 130 species belonging to the subfamily have been grouped into complexes and subcomplexes, based mainly on similarities in their morphology and geographic distribution.

The Triatoma sordida subcomplex belongs to the T. infestans complex; it is integrated by $T$. sordida, T. garciabesi, T. guasayana and T. patagonica, a group of species differentially distributed in Argentina, Bolivia, Brasil and Paraguay. The insects are found mainly in sylvatic and peridomestic habitats, but tend to colonize the houses after erradicating the main vectors $T$. infestans and $T$. brasiliensis. They show a high infection rate with $T$. cruzi, thus they are considered as secondary vectors and potentially risky species by the control programmes. The specific status of some members of the subcomplex has been put in doubt mainly due both to their high variability in many phenotypic features and to the possibility to obtain viable offspring after experimental crossings; this is especially true for $T$. garciabesi and T. patagonica. Furthermore, some papers report a cryptic speciation process in T. sordida.

$T$. dimidiata is one of the three major vectors of Chagas disease, together with $T$. infestans and Rhodnius prolixus, being the main vector in both North and Central America. With a high infection rate with $T$. cruzi, the species is highly adaptable to various environmental conditions and easily colonize the peridomestic and domestic habitats from its sylvatic reservoirs; in addition, insects have also been found in big cities. Probably originated in Central America, T. dimidiata actually has a wide distribution from Central Mexico throughout almost all countries of Central America, to Colombia, Venezuela, Peru and Ecuador in South America. It was initially separated in three subspecies, based mainly on its morphological and ethological variability along its geographic distribution; but afterwards reclassified as a highly variable species in one of the most comprehensive studies on triatomines taxonomy. However, a number or recent studies using genetic and phenetic characters, showed a level of variability difficult to place into a single species. 
Phenotypic (cytology, morphometry, isoenzymes, etc) and genetic (RAPD, rDNA ITS-1 and 2, 18S RNA techniques) characters, together with multivariate analysis techniques, have been widely used in taxonomic studies on several groups of animals, plants, fungi and bacteria, helping the classical taxonomy to clarify relationships between cryptic groups. The insect cuticular hydrocarbon pattern has been used as taxonomic character for several groups, many of them with medical importance, such as Glossina sp., Simulium sp. and Anopheles sp. genera. The hydrocarbon pattern of several species of triatomines is known, and its structure was also determined for T. infestans, T. mazzottii and R. prolixus.

In this study, the cuticular hydrocarbon analysis of the $T$. sordida subcomplex allowed us to differentiate T. garciabesi and T. sordida as distinct species, however, there is no distinctive hydrocarbon character discriminating $T$. patagonica, thus remaining as an Argentine population of $T$. guasayana. The latter appears as the most differentiated species of the subcomplex, with $T$. garciabesi and $T$. sordida closely related. In agreement with other taxonomic techniques, $T$. sordida splitted in two clearly differentiated groups, which could be considered as distinct subspecies.

The hydrocarbon analysis on $T$. dimidiata showed that it is necessary a major reconsideration of its taxonomical status. Most of the populations clustered in three neatly differentiated groups, which correspond to the original classification in subspecies: $T$. dimidiata maculipennis for most of the Mexican specimens, $T$. d. dimidiata for the Central American ones and T. d. capitata for most of the South American insects. Another group was obtained, comprising the specimens from the Mexican state of Yucatan and Northern Guatemala (Peten); it may constitute another species or subespecies, as also showed by morphometric and genetic analyses. Specimens collected in the cave system of Lanquin (Guatemala) and Augustine (Belice) showed a high degree of differentiation, only comparable to that obtained for other species.

Results of this thesis show that the cuticular hydrocarbon pattern is a useful and sensitive taxonomic marker both to study the interspecific relationship in triatomins and to evaluate their intraspecific variability. It allows inferring migration of insects between either geographic regions or habitats, therefore providing useful information to the control programmes in designing specific strategies. 


\section{Introducción}

\section{1. Enfermedad de Chagas: características generales, epidemiología y distribución.}

La Enfermedad de Chagas o Tripanosomiasis americana, es una infección parasitaria causada por el protozoo flagelado Trypanosoma cruzi Chagas 1909 (Protozoa: Kinetoplastida) y transmitida al hombre principalmente mediante las deyecciones de los insectos hematófagos de la subfamilia Triatominae Jeannel 1919 (Hemiptera: Reduviidae).

Se considera que era una enfermedad originalmente zoonótica, transmitida por los triatominos entre distintos animales silvestres y restringida a ese ambiente (Vinhaes y Dias 2000). La posible alteración de los ciclos de transmisión selvática debido a la ingresión del hombre en los ambientes silvestres con prácticas agrícolo-ganaderas y de recolección, junto a la introducción de los triatominos al hábitat humano (casas, corrales y gallineros) pueden haber posibilitado el inicio del ciclo de infección doméstica de la enfermedad.

El ciclo de transmisión se inicia cuando estos insectos incorporan el parásito en su tracto digestivo al tomar sangre de un organismo infectado; luego al tomar sangre de un segundo huésped, eventualmente defecan sobre la piel y/o membranas mucosas del mismo, desde donde el parásito penetra al torrente sanguíneo iniciando la infección (figura 1). Las transfusiones sanguíneas, la vía transplacentaria y la ingestión constituyen otras formas de transmisión de menor incidencia.

Figura 1. Ciclo de transmisión vectorial de la enfermedad de Chagas

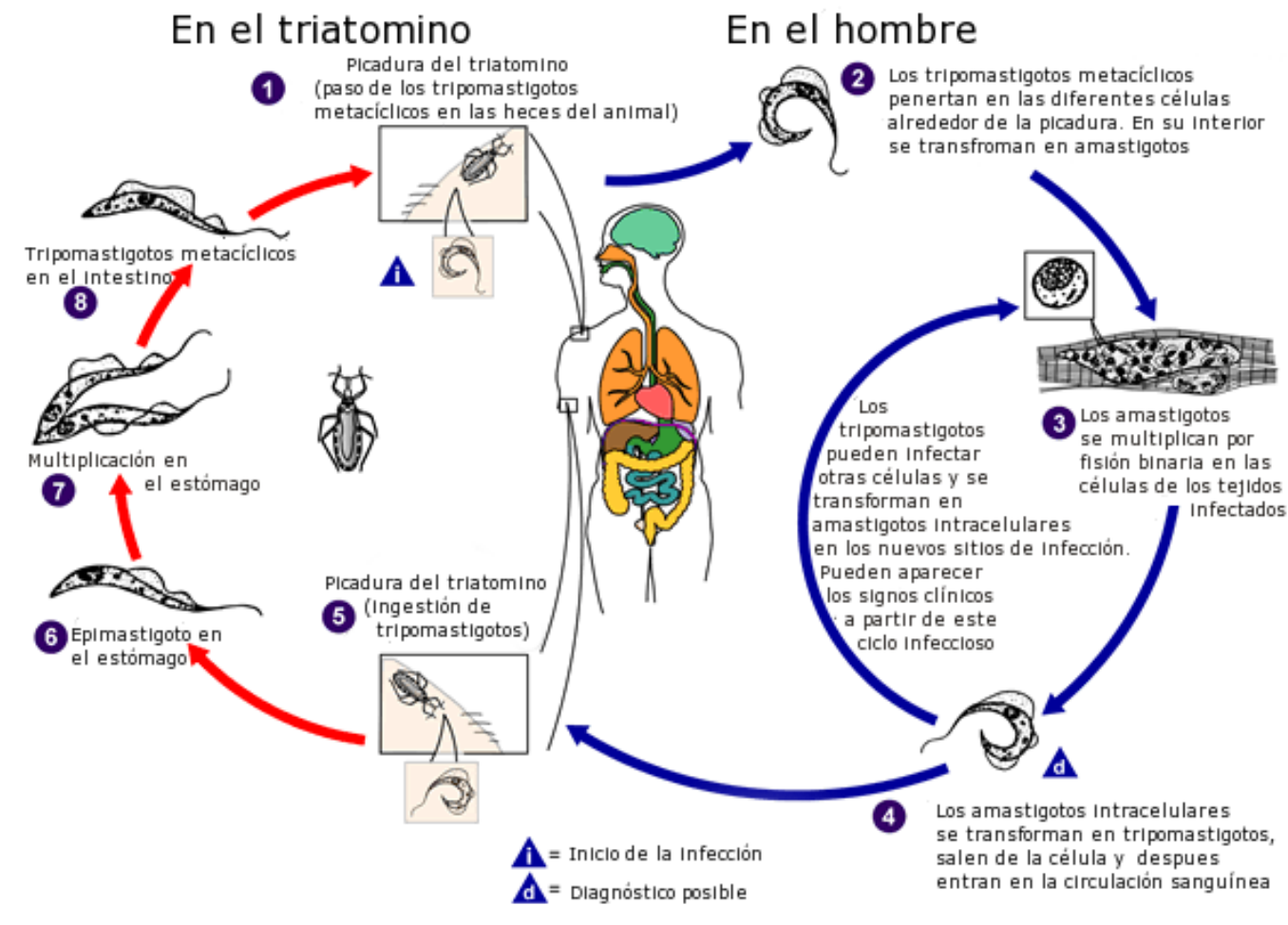


La enfermedad se encuentra principalmente localizada en América Latina, desde el centro de Mexico hasta el centro de Argentina, siendo una de las cuatro principales endemias de la región. Las estimaciones actuales indican que 13 millones de personas se encuentran infectadas, con una incidencia anual de 200.000 casos en quince países (Morel y Lazdins 2003, Schofield et al. 2006).

Dado que no se ha podido desarrollar hasta el momento una vacuna contra esta enfermedad, y las terapias de cura están acotadas a los estadios iniciales de la misma, la mayor parte de los esfuerzos de control están principalmente orientados a la comprensión de los aspectos biológicos de los triatominos y a su eliminación de los ambientes donde coexisten con el hombre (hábitats domésticos y peridomésticos).

\section{2. Triatominos: características morfológicas y biológicas, clasificación taxonómica}

Los triatominos (Hemíptera: Reduviidae: Triatominae) son insectos pertenecientes al orden Hemíptera, es decir, que tienen el primer par de alas convertido en hemiélitros mientras que el segundo es enteramente membranoso. Son hematófagos, alimentándose normalmente de la sangre de diversos mamíferos y aves, y más raramente de la sangre de otros vertebrados y de la hemolinfa de insectos. La mayor parte de las especies se distribuyen en casi todos los países de América Latina, desde el norte de Mexico hasta el norte de la patagonia Argentina, existiendo algunas localizadas en Europa (Triatoma rubrofasciata De Geer 1773) y Asia (T. cavernicola Else y Cheong 1977 y otras, género Linshcosteus sp. Distant 1904) (Gorla et al. 1997). Las hipótesis vigentes sugieren que los triatominos evolucionaron a partir de una o más formas de redúvidos predadores, pero la ausencia de registro fósil impide el reconocimiento de este/estos grupo/s (Lukashevich y Mostovski, 2003).

Sus principales detalles corporales se muestran en la figura 2.

Figura 2. Detalles corporales principales de un triatomino.

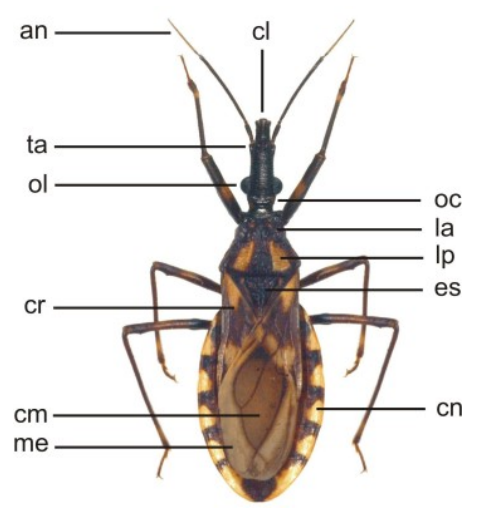

an: antena, cl: clípeo, ta: tubérculo antenal, ol: ojos, oc: ocelos, la: lóbulo anterior del pronoto, lp: lóbulo posterior del pronoto, es: escutelo, cr: corion, cn: conexivo, cm: celda de la membrana alar, me: membrana alar. 
Su cabeza consta de una proboscide que utiliza para succionar la sangre de sus presas y cuya disposición y longitud constituyen un carácter que permite diferenciar a los triatominos de otros redúvidos de alimentación fitófaga o predadora (figura 3).

Figura 3. Proboscides de redúvidos, A: fitófago, B: predador, C: hematófago
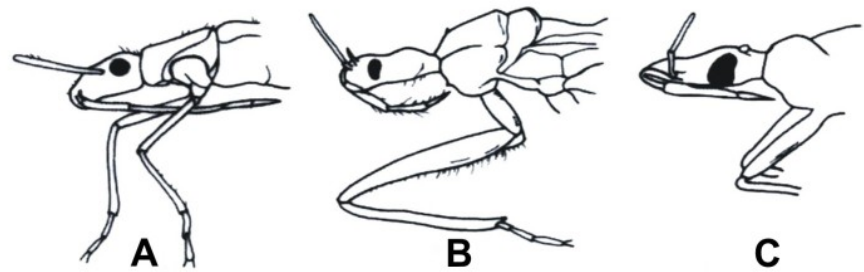

La cabeza presenta además un par de antenas de función sensorial ubicadas cada una sobre un tubérculo antenífero cuya inserción en la cabeza permite distinguir los tres principales géneros de triatominos Panstrongylus sp. Berg 1879, Rhodnius sp. Stal 1859 y Triatoma sp. Laporte 1833 (figura 4)

Figura 4. Posiciones de los tubérculos anteníferos en 3 géneros de triatominos. A: Panstrongylus sp. con cabeza corta y tubérculo cerca de los ojos, B: Rhodnius sp. con cabeza larga y estrecha y tubérculo cerca del extremo anterior, C: Triatoma sp. con el tubérculo en el medio de la región anteocular.

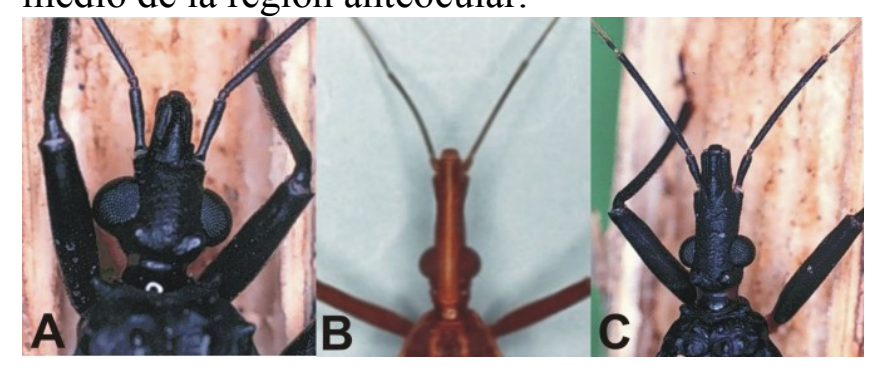

El tórax, de manera característica en los insectos, tiene tres segmentos pro, meso y metatórax donde se insertan los tres pares de patas y los dos pares de alas, las primeras como hemiélitros.

El abdómen es deprimido con una banda perimetral llamada conexivo donde se alternan patrones de coloración que en algunos casos permiten la identificación de la especie.

A partir del huevo, se suceden 5 estadíos ninfales y el estado adulto (figura 5), siendo todos hematófagos. La duración del ciclo vital es muy variable en cuanto a la especie considerada pudiendo encontrarse una o dos generaciones anuales. 
Figura 5. Ciclo vital de los triatominos.

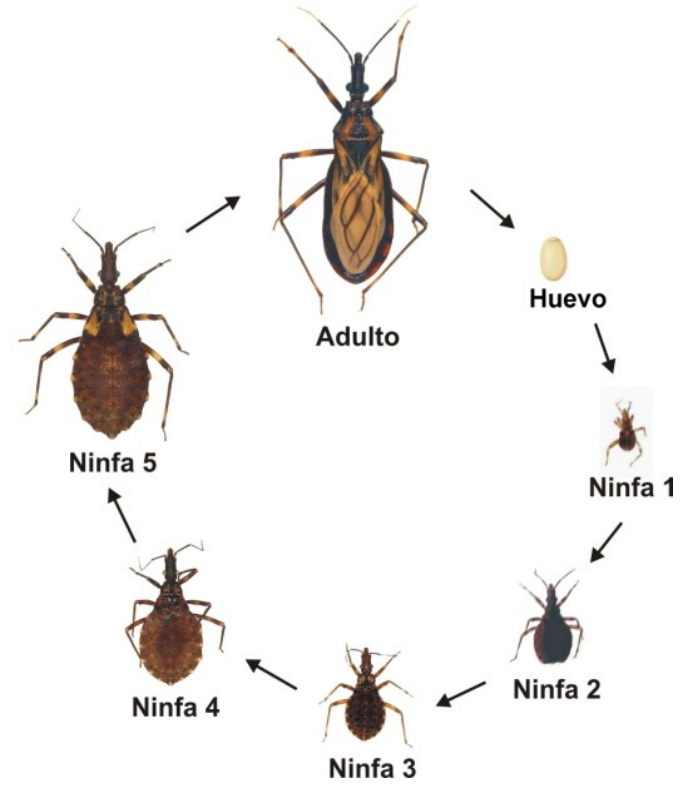

Según revisiones recientes (Galvão et al. 2003), las 137 especies que cuenta la subfamilia Triatominae se reparten en 6 tribus según la tabla 1.

Tabla 1. Tribus y géneros de la subfamilia Triatominae

\begin{tabular}{|c|c|c|}
\hline Tribus & Géneros & $\mathrm{N}^{0}$ de especies \\
\hline Alberproseniini & Alberprosenia & 2 \\
\hline \multirow[t]{4}{*}{ Bolboderini } & Belminus & 6 \\
\hline & Bolbodera & 1 \\
\hline & Microtriatoma & 2 \\
\hline & Parabelminus & 2 \\
\hline \multirow[t]{2}{*}{ Cavernicolini } & Cavernicola & 2 \\
\hline & Torrealbaia & 1 \\
\hline Linshcosteini & Linshcosteus & 6 \\
\hline \multirow[t]{2}{*}{ Rhodniini } & Psammolestes & 3 \\
\hline & Rhodnius & 16 \\
\hline \multirow[t]{9}{*}{ Triatomini } & Dipetalogaster & 1 \\
\hline & Eratyrus & 2 \\
\hline & Hermanlentia & 1 \\
\hline & Meccus & 6 \\
\hline & Mepraia & 2 \\
\hline & Nesotriatoma & 3 \\
\hline & Panstrongylus & 13 \\
\hline & Paratriatoma & 1 \\
\hline & Triatoma & 67 \\
\hline
\end{tabular}

Según Galvão et al. 2003 
En base a similitudes morfológicas, ecológicas y de distribución geográfica, las especies se han agrupado en complejos y subcomplejos. Las especies del subcomplejo $T$. sordida integran el complejo $T$. infestans, grupo ampliamente distribuído en Argentina, Bolivia, sur de Brasil, Paraguay, Perú y Uruguay y que coloniza una amplia variedad de hábitats silvestres, peridomésticos y domésticos. Triatoma dimidiata Latreille 1811 ha sido tradicionalmente incluida en el complejo phyllosoma, grupo de especies con amplia distribución en Mexico, aunque actualmente esta pertenencia esta discutida.

\section{3. Características biológicas del subcomplejo Triatoma sordida}

Según los diversos autores que lo han descripto y estudiado (Lent y Wygodzinsky 1979, Oscherov et al. 1998, Canale y Stariolo 2002) el subcomplejo T. sordida es un grupo de especies distribuidas principalmente en el centro y norte de Argentina, Bolivia, Paraguay y sur de Brasil, que presentan una alta semejanza morfológica, especialmente en los estadios ninfales. Son ejemplares de tamaño mediano a pequeño (aprox. $20 \mathrm{~mm}$ las hembras y $18 \mathrm{~mm}$ los machos), de color claro y de hábitat generalmente silvestre y peridoméstico (figura 6). Tienen un ciclo de vida de aprox. 300 días. Se las encuentra naturalmente infectadas con $T$. cruzi en alta frecuencia. Está compuesto por las siguientes especies:

-Triatoma guasayana Wygodzinsky y Abalos 1949: los adultos son de color claro, con conexivo ancho que presenta manchas transversales muy angostas, estranguladas en su centro, fémures claros con anillo apical oscuro, trocánter, coxas, lóbulo posterior del pronoto y escutelo totalmente oscuros. Las ninfas son pardo negruzcas, con el conexivo de cada urito oscuro en la base con un triángulo blanco en forma de media luna en el centro, patas con fémur anillado y coxas oscuras. Se encuentra en Bolivia, Paraguay y Argentina, en este último llega desde el noreste hasta la provincia de La Pampa. Los adultos de la región oriental de Bolivia presentan una gran diferenciación morfológica siendo aproximadamente $0.5 \mathrm{~cm}$. más anchos en la región abdominal que los otros integrantes de la especie. En hábitats silvestres se la encuentra en cactus huecos, troncos secos y caídos, entre piedras, bajo la corteza de los árboles, asociada a nidos de aves, roedores y anuros. En hábitats peridomésticos se la encuentra asociada persistentemente a corrales de cabras, vacunos y cerdos; también en gallineros, palomares y refugios de perros; corteza de árboles y material apilado. No suele colonizar la vivienda humana, sino que, debido a su facilidad para volar, ingresa a ella desde el peridomicilio, atraida por la luz y luego de alimentarse se retira. Es de comportamiento agresivo y se la encontró naturalmente infectada con $T$. cruzi en numerosas oportunidades.

-Triatoma sordida Stal 1859: los adultos son de color claro, con conexivo angosto, claro, con manchas transversales un poco mas anchas que en $T$. guasayana; pronoto con una mancha 
clara en cada ángulo posterior; fémures claros con anillo apical oscuro; proceso del escutelo y coxas claras. Las ninfas son pardo rojizas con el conexivo de cada urito de color oscuro atravesado por una banda clara; patas con fémur anillado, coxas claras. Se encuentra en Bolivia, Brasil, Paraguay, Uruguay y Argentina, en este último se distribuye en las provincias de Salta, Misiones, Corrientes, Formosa, norte de Chaco y Santa Fe. En hábitats silvestres se la encuentra asociada a refugios de marsupiales y roedores, troncos huecos, cortezas de árboles y en palmeras, bromelias y nidos de aves. En hábitats peridomésticos se la encuentra en gallineros, palomares, conejeras y en sitios frecuentados por ratas. Invade frecuentemente la vivienda humana y puede encontrarse en zonas urbanas. En Brasil ha reemplazado a $T$. infestans Klug 1834 luego de la erradicación de esta especie de los domicilios (Zeledón 1983) -Triatoma garciabesi Carcavallo, Martínez, Cichero, Prosen y Ronderos 1967: es morfológicamente muy semejante a $T$. sordida, tanto en el adulto como en los estadios ninfales; tiende a ser mas pequeña y oscura que ésta última. Está descripta solo en Argentina, en la región del noroeste, provincias de Salta, Jujuy, Tucumán, Santiago del Estero, Córdoba, Catamarca, La Rioja, San Juan, Mendoza, Santa Fe, noroeste de Formosa y noroeste y centro de Chaco. En hábitats silvestres es frecuente en nidos de aves, madrigueras de roedores, árboles secos y bajo la corteza de árboles. En hábitats peridomésticos se la encuentra persistentemente bajo la corteza de árboles donde duermen gallinas, gallineros y corrales de cabras y cerdos, material apilado. Tiene baja tendencia a invadir la vivienda humana.

-Triatoma patagonica Del Ponte 1929: son de color negro, con conexivo con manchas transversales claras; hemiélitros con mancha clara en la base, pronoto negro uniforme, patas negras con estrecho anillo apical amarillo. Las ninfas son en general negras y presentan en el conexivo una mancha clara entre dos oscuras, que contrasta con el resto del cuerpo que es totalmente negro. Está descripta solo en Argentina, su distribución se extiende desde Salta hasta el noroeste de Santa Cruz. En hábitats silvestres se la encuentra entre piedras, bajo troncos y en cuevas de roedores. En el peridomicilio suele ocupar gallineros, palomares y corrales. Es atraida facilmente por la luz, no coloniza la vivienda humana pero entra en ella para alimentarse y luego se retira. Es buena voladora y de comportamiento agresivo.

La importancia epidemiológica de este grupo de insectos está dada por su amplio reservorio silvestre, el encontrarse naturalmente infectada con $T$. cruzi, así como la posibilidad de colonizar viviendas humanas, especialmente luego de la erradicación de $T$. infestans (Lent y Wygodzinsky 1979, Zeledón 1983, Canale y Stariolo 2002). 
Figura 6. Ejemplares del subcomplejo T. sordida.

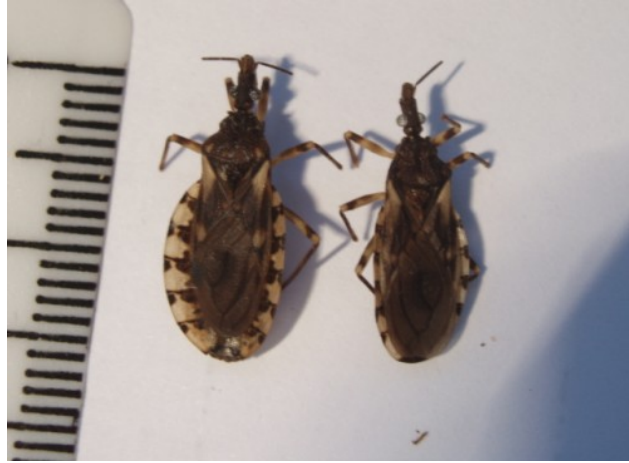

T. sordida Argentina (hembra y macho)

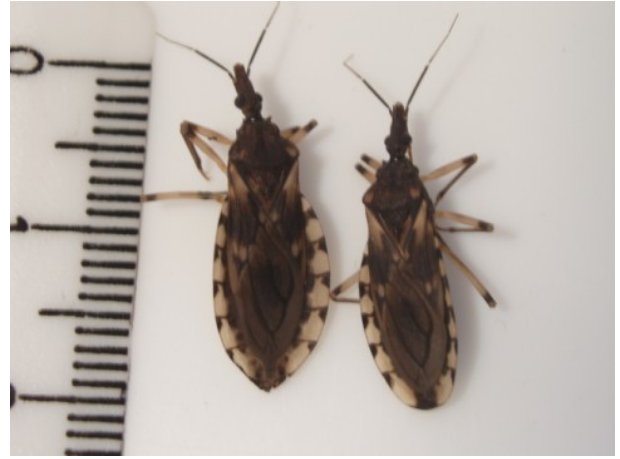

T. garciabesi Argentina (hembra y macho)

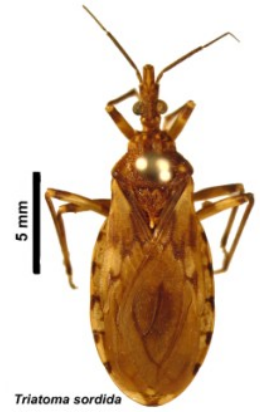

T. sordida Brasil macho

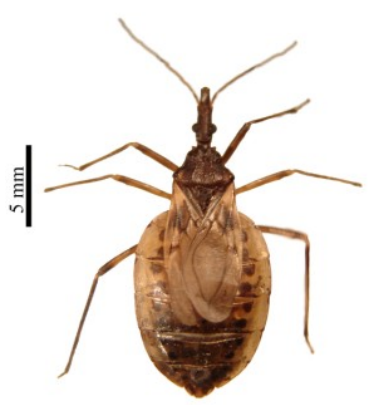

T. guasayana Bolivia hembra

\section{4. Características biológicas de Triatoma dimidiata}

Triatoma dimidiata Latreille 1811 es el tercer vector de la enfermedad de Chagas en orden de importancia, luego de Triatoma infestans y Rhodnius prolixus Stal 1859 (Dujardin et al. 2000). Es un insecto grande, los adultos llegan a medir aproximadamente $70 \mathrm{~mm}$, de abdomen ancho y de colores claros con tonos marrones y amarillos. Son de color negro, conexivo anaranjado con manchas transversales oscuras; hemiélitros con mancha clara en la base, pronoto y patas de color negro uniforme (figura 7)

Figura 7. Ejemplar de T. dimidiata

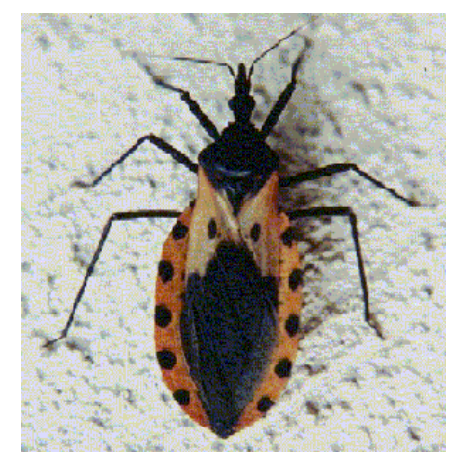


Tiene una amplia distribución geográfica, desde el norte de Mexico, a través de casi todos los países de Centroamérica, y en la región noroeste de Sur América (Venezuela, Colombia, Ecuador y Peru) (Lent y Wygodzinsky 1979). Se la encuentra en una gran variedad de ecosistemas que van desde los bosques premontanos calurosos y húmedos, como en Costa Rica, hasta regiones tropicales secas como la costa pacífica de Guatemala; con una distribución altitudinal que va desde los 0 a los $2000 \mathrm{msnm}$. En ambientes silvestres, se encuentran especímenes bajo árboles caídos y piedras, en nidos de aves, hojas de palmeras, madrigueras de diversos mamíferos como armadillos, roedores y marsupiales, cuevas de murciélagos, etc (Petana 1971, Zeledón 1981, Bustamante et al. 2004). Ha mostrado una gran facilidad para invadir el ambiente peridoméstico desde el silvestre (Monroy et al. 2003a) colonizando gallineros y corrales y asimismo el ambiente doméstico, a donde son atraídas por la luz. Sus distribución en los ambientes silvestres, peridomésticos y domésticos es variable; en Bélice y el norte de Guatemala se la encuentra principalmente ambientes silvestres, mientras que en el sur de Guatemala la especie presenta una alto grado de domesticidad. Hay numerosos reportes sobre un constante flujo de insectos entre hábitats, en distintas regiones de Costa Rica, Mexico, Guatemala y Colombia pudiendo encontrarse tanto insectos en ambientes peridomésticos alimentados con sangre humana, como insectos dentro de las viviendas humanas alimentados con sangre de animales silvestres (Zeledón et al. 1973). Dentro de las viviendas humanas, los insectos se ubican preferentemente en la parte inferior de las paredes, cercana al zócalo y en los pisos, donde pasan más desapercibidos (Monroy et al. 2003b). Estudios realizados en la península de Yucatán muestran que los adultos tienen la tendencia de volar e invadir las casas en la estación cálida y seca del año, a partir de lo cual pueden formar poblaciones domésticas estables (Dumonteil et al. 2002). Se ha observado en las ninfas encontradas en las viviendas un comportamiento de "camouflage", en el que se cubren con tierra para pasar desapercibidas. Su tendencia a colonizar los domicilios no solo se restringe a zonas rurales y semi rurales sino que han sido encontrados insectos en viviendas urbanas de las ciudades de Merida (Mexico), Caracas (Venezuela), Tegucigalpa (Honduras), San Jose y Heredia (Costa Rica) y Guayaquil (Ecuador) (Dorn et al. 2007). Debido a su alta tasa de infección natural con T. cruzi, así como su gran reservorio silvestre, esta especie presenta una gran importancia epidemiológica (Schofield 2002).

\section{5. Hidrocarburos cuticulares en insectos}

Los hidrocarburos (HC) son constituyentes característicos de los lípidos cuticulares de insectos (Blomquist et al. 1972, Soliday et al. 1974, Blomquist y Jackson 1979, Lockey 1980, 
Warthen y Uebel 1980, Howard y Blomquist 1982, Blomquist y Dillwith 1985, Lockey 1985a, Blomquist et al. 1987, Lockey 1988).

Su biosíntesis tiene como eje la síntesis de ácidos grasos y su posterior elongación. Kolattukudy et al (1976) probaron en plantas que el ácido C16:0 producido por una ácido graso sintetasa citosólica, es elongado mediante una serie de acil-CoA elongasas relacionadas a la membrana del retículo endoplásmico, hasta producir un acil-CoA de muy larga cadena, el cual el decarboxilado para obtener un hidrocarburo con un átomo de carbono menos. En insectos, la biosíntesis de los ácidos grasos de muy larga cadena (AGMLC) en el integumento, fue descripta por primera vez en $T$. infestans y ocurre también a través de la acción de una ácido graso sintetasa acoplada a acil-CoA elongasas microsomales (Juárez y Brenner 1989, Juárez 1995, Juárez et al. 1996). Estos estudios demostraron que las tres especies lipídicas principales de la cutícula de este insecto, hidrocarburos, alcoholes grasos y ácidos grasos libres o esterificados, están relacionados biosintéticamente; y que los ácidos grasos de 26, 28, 30, 32 y 34 carbonos en la cadena lineal son los sustratos para la síntesis de los principales hidrocarburos $(n \mathrm{C} 25, n \mathrm{C} 27, n \mathrm{C} 29, n \mathrm{C} 31 \mathrm{y} n \mathrm{C} 33)$. Este último proceso ocurriría mediante una vía de decarboxilación/decarbonilación reductiva (Reed et al. 1994, Schneider-Behaddad y Kolattukudy 2000, Blomquist 2003). Además de cadenas lineales, AGMLC ramificados de 16 y 18 carbonos en la cadena lineal fueron detectados en pequeñas cantidades en el integumento de Blattella germanica Linnaeus 1767 y T. infestans, los cuales son precursores de los hidrocarburos ramificados (Juárez et al. 1992, 1996).

En los insectos, los hidrocarburos cuticulares varían cualitativa y cuantitativamente entre las especies así como entre sexos y distintos estadíos de desarrollo dentro de la misma especie. Pueden encontrarse en pequeñas proporciones como en el plecóptero Pteronarcys californica Newport 1848 (Armold et al. 1969) donde constituyen el $3 \%$ de los lípidos cuticulares, hasta constituir el componente mayoritario de los mismos como en los blatodeos Periplaneta japonica Karny 1908 y Periplaneta americana Linnaeus 1758 (85 \% y 90 \% respectivamente) (Jackson 1972), los ortópteros Acheta domesticus Linnaeus 1758 (85 \%-89 \%) (Warthen y Uebel 1980) y Locusta migratoria Linnaeus 1758 (78 \%-92\%) (Genin et al. 1986) y el himenóptero Bombyx mori Linnaeus 1758 (78 \%) (Hadley et al. 1981). Usualmente se encuentran como mezclas complejas de componentes saturados de cadena lineal (nalcanos), insaturados (n-alquenos) y metil ramificados (r-alcanos).

\section{$\underline{n \text {-Alcanos }}$}

Estos hidrocarburos se encuentran en un rango muy variable de proporciones; en Drosophila pseudoobscura Frolova y Astaurov 1929 y D. virilis Sturtevant 1916 (Blomquist 
et al. 1985, Jackson y Bartelt 1986) constituyen solo el $2 \%$ y $5 \%$ respectivamente, mientras que en tenebriónidos del género Eurychora sp. Fabricius 1785 llegan al 98 \% de la fracción de hidrocarburos (Lockey 1985b).

En la mayoría de las especies estudiadas, los $n$-alcanos se encontraron como series contínuas homólogas de longitud variable, tal como la serie de $n \mathrm{C} 27$ a $n \mathrm{C} 31$ en la cucaracha Arenivaga investigata Friauf y Edney 1969 (Jackson 1983). Sin embargo, algunas especies poseen series compuestas totalmente de $n$-alcanos impares, como en el género Melanoplus sp. Stal 1873 donde la serie impar va desde $n$ C23 a $n$ C49 (Jackson 1981). Usualmente predominan las cadenas impares de $n \mathrm{C} 23$ a $n \mathrm{C} 33$.

\section{$\underline{n \text {-Alquenos }}$}

La proporción de $n$-alquenos es variable a lo largo de las especies estudiadas, pudiendo ir desde el $0.8 \%$ de los lípidos cuticulares en el odonato Sympetrum danae (Sulzer 1776) (Jacob y Hanssen 1979) hasta el 68 \% como en el himenóptero Pikonema alaskensis Ross 1937 (Bartelt et al. 1984). Los monoalquenos usualmente son más frecuentes que los alcadienos o alcatrienos.

La termitas Reticulitermes flavipes Kollar 1837 y Zootermopsis sp. Emerson 1933 tienen sólo uno y dos monoalquenos respectivamente ( $n$-pentacoseno la primera, $n$-tricoseno y $n$-hentriaconteno la segunda) (Howard et al. 1978, Haverty et al. 1988) y con proporciones variables en las distintas castas, mientras que en la termita Macrotermes subhyalinus Rambur pueden llegar a contarse cerca de 20 alquenos los que constituyen hasta el $65 \%$ de los hidrocarburos cuticulares (Kaib et al. 2004). En Apis mellifera Linnaeus 1758 estos forman una completa serie homóloga de 23 a 35 carbonos, constituyendo hasta el $47 \%$ de los lípidos cuticulares (Blomquist et al. 1980). La posición mas común de la insaturación es en el carbono 9 de la cadena, aunque se pueden encontrar complejas mezclas de isomeros, como en Tenebrio molitor Linnaeus 1758 que presenta una mezcla isomérica desde 8-enos a 16-enos (Lockey 1978). Los alcadienos son menos comunes que los monoenos. En la cucaracha $P$. americana el 6,9-heptacosadieno se encuentra como el único hidrocarburo insaturado, estando además en una alta proporción (71 \%) (Jackson 1972); pueden encontrarse además como series, como en D. pseudoobscura que presenta series de 5,9-, 7,11-, 9,18-, 9,20-y 9,11dienos que van desde 25 a 35 carbonos (Blomquist et al. 1985).

Los alcatrienos son también poco comunes. En D. pseudoobscura comprenden el 9.2 $\%$ de los lípidos cuticulares y van desde 25 a 37 carbonos, los mas abundantes son los 5,9,17y 5,9,19- trienos de C29 y C31 (Blomquist et al. 1985). En P. alaskensis comprenden el $1.1 \%$ 
y el $0.1 \%$ de los lípidos cuticulares de los machos y las hembras respectivamente, encontrándose como 3,6,9-trienos desde 25 a 29 carbonos (Bartelt et al. 1984).

\section{$\underline{\text { Alcanos ramificados }}$}

Los lípidos cuticulares de la mayoría de las especies estudiadas contienen mezclas isoméricas de metilalcanos, los cuales se encuentran como mono y dimetilalcanos y mas raramente como trimetil y tetrametilalcanos.

\section{$\underline{\text { Monometilalcanos }}$}

A los monometilalcanos usualmente se los divide en terminales, si la ramificación se encuentra entre el carbono 2 al 4 de la cadena lineal, o internos si se encuentra desde la posición 5 hacia atrás.

La distribución de los monometilalcanos terminales varia ampliamente entre las especies pertenecientes a un grupo determinado de insectos. En Ortópteros de los géneros Anabrus sp. Haldeman 1852, Acheta sp., Nemobius sp. Serville 1839 y Gryllus sp. Linnaeus 1758 (Blomquist et al. 1976, Jackson y Blomquist 1976) así también como en Melanoplus sp. (Jackson 1981, Nelson et al. 1984) se encuentran 2- y 3-metilalcanos pudiendo estar ambos presentes o faltar alguno de ellos mientras que en Schistocerca americana (Drury 1773), S. gregaria Forskal 1775 y S. vaga (= Schistocerca nitens Thunberg 1815) (Nelson y Sukkestad 1975, Lockey 1976, Jackson 1982) se encuentran ausentes. Se encuentran también extensas series homólogas de 2- y 3-metilalcanos en dípteros de los géneros Glossina sp. Wiedemann 1830 y Musca sp. Linnaeus 1758 (Nelson et al. 1981, Nelson y Carlson 1986), así como en odonatos de los géneros Aeschna sp. Fabricius 1775 y Sympetrum sp. Newman 1833 (Jacob y Hansen 1979).

La mayoría de las especies de insectos estudiadas presentan monometilalcanos terminales e internos, que casi invariablemente se encuentran como mezclas isoméricas. En los isómeros con número par de átomos de carbono las ramificaciones tienden a estar en posiciones impares de la cadena, mientras que en las cadenas impares las ramificaciones pueden estar tanto en posiciones pares como impares (Lockey 1988). Estos compuestos pueden estar limitados a unos pocos isómeros como en la termita $R$. flavipes (Howard et al. 1978), la cuál solo presenta el 5-metil-C25 o la abeja A. mellifera (Blomquist et al. 1980) que comprende los 5-, 11- y 13- metil isómeros de C27 y C29. Series extensas de monometilalcanos internos pueden encontrarse en especies de los géneros Melanoplus sp. y Locusta sp. Linnaeus 1758 (Nelson et al. 1984, Genin et al. 1986) con cadenas de C26 a C50 con ramificaciones metilo en posiciones 5- a 18- en el primero, o con cadenas de C28 a C38 
con ramificaciones en posiciones 11- a 19- en el segundo. Los monometilalcanos internos pueden representar desde el $0.3 \%$ a $0.9 \%$ como en D. pseudoobscura (Blomquist et al 1985) y M. subhyalinus (Kaib et al. 2004) hasta el $49.5 \%$ como en la cucaracha A. investigata (Jackson 1983).

\section{Dimetilalcanos}

La mayoría de las especies presentan series de cadenas pares e impares de dimetilalcanos aunque las cadenas impares suelen predominar. Del mismo modo que los monometilalcanos, éstos se presentan casi siempre como mezclas isoméricas en las cuales ambas ramificaciones pueden estar separadas por 3 a 15 grupos metileno. En las cadenas con número par de átomos de carbono predominan las ramificaciones en posiciones pares, en las de número impar los grupos metilo se insertan en posición impar. La mezcla de isómeros puede ser escasa, con pocas series homólogas o llegar hasta cerca de cuarenta dimetil isómeros como en el grillo Melanoplus packardii Scudder 1878 (Nelson et al 1984) con cadenas de 28 a 55 carbonos. En Musca domestica Linnaeus 1758 (Nelson et al. 1981) las ramificaciones van desde la posición 3- a la 17- de la cadena, por ejemplo mezclas de 3,7-, 3,9-, 3,11-, 3,13-, 9,13-, 11,15- y 13,17-dimetil C29 y 4,10-, 4,12, 4,14 y 10,14-dimetil C30. En el fásmido Diapheromera femorata Say 1824 se encontraron mezclas de 9,13-, 11,15-, 13,17-, 15,19-17, 21- y 19,23- dimetil isómeros de cadenas impares con 33 a 47 carbonos (Warthen et al. 1981). Los dimetilalcanos pueden ser un componente escaso de los hidrocarburos cuticulares o constituir una gran proporción de los mismos como en hembra de la mosca tse-tse Glossina pallidipes Austen 1903 en la cual comprenden cerca del $75 \%$ (Nelson y Carlson 1986).

\section{$\underline{\text { Trimetilalcanos }}$}

A diferencia de lo que ocurre con los dimetilalcanos, en los trimetilalcanos reportados en la mayoría de las especies analizadas las cadenas con número par de átomos de carbono suelen predominar sobre las de número impar; en algunas especies, como la langosta $S$. americana (Jackson 1982), solo se encuentran cadenas pares mientras que en otros insectos como la mosca tse-tse Glossina austeni Newstead 1912 (Nelson y Carlson 1986) se detectaron series homólogas contínuas de 34 a 41 átomos de carbono en la cadena. De manera común, las ramificaciones están separadas por tres grupos metilo (Lockey 1988) siendo los isómeros 9,13,17-, 11,15,19-, 13,17,21- y 15,19,23- los más usuales. 


\section{$\underline{\text { Tetrametilalcanos }}$}

Estos hidrocarburos ramificados son infrecuentes en los insectos, generalmente consisten en componentes minoritarios o incluso trazas (Nelson et al. 2002). Los hidrocarburos 3,7,11,15-tetrametil C30 y el 4,8,12,16-tetrametil C32 son componentes importantes de la cuticula de Glossina brevipalpis Newstead 1911 (Nelson et al. 1988), siendo minoritarios en otras moscas de este género. En el lepidóptero Lymantria dispar Linnaeus 1758 se encontró el isómero 4,8,12,16- en cadenas pares de 30 a 34 carbonos (Jurenka y Subchev 2000).

De los lípidos que componen la epicutícula de los insectos, los hidrocarburos serían los principales responsables de conferirle a ésta su característica de impermeabilidad (Hadley 1978). Diversos experimentos han demostrado que la abrasión de la cutícula con sustancias capaces de dañar la capa de lípidos o la remoción de la misma con solventes orgánicos tienen efectos drásticos en la supervivencia de los insectos debido a la pérdida de agua (Beamont 1945, Wigglesworth 1945, Locke 1965, Sponsler y Appel 1990, Juárez 1994a,b). Los lípidos cuticulares contribuirían además a modular la tasa de ingreso de insecticidas (Juárez 1994a). Con respecto al ingreso de microorganismos, se ha demostrado en $T$. infestans y otros insectos plaga de granos almacenados, que los lípidos cuticulares y en particular los hidrocarburos, pueden actuar como fuente de carbono primaria para el desarrollo de las primeras etapas de crecimiento y penetración de hóngos entomopatógenos como Beauveria bassiana (Balsamo) Vuillemin y Metarhizium anisopliae (Metchnikoff) Sorokin (Napolitano y Juárez 1997, Crespo et al. 2000, Crespo et al. 2002, Crespo 2006, Pedrini 2006).

\section{6. Hidrocarburos cuticulares en triatominos}

Junto con los alcoholes grasos, los hidrocarburos constituyen los lípidos mayoritarios de la cutícula de los triatominos. Esta composición lipídica con predominio de cadenas hidrocarbonadas largas e inertes, contribuiría a las características de impermeabilidad de la cutícula de estos insectos, los cuáles muestran una notable adaptación a ambientes secos. En Triatoma infestans (Juárez et al. 1984) los hidrocarburos comprenden aproximadamente el 21 $\%$ de los lípidos cuticulares en los adultos, llegando al $37 \%$ en las ninfas, siendo así en estas últimas el componente mayoritario; en otros triatominos esta composición resultó similar (Juárez y Brenner 1987).

En la figura 8 se muestran perfiles de hidrocarburos correspondientes a distintos géneros de triatominos (T. infestans, Panstrongylus megistus Burmeister 1835 y R. prolixus). 
Figura 8. Perfiles de hidrocarburos cuticulares de 3 especies de triatominos
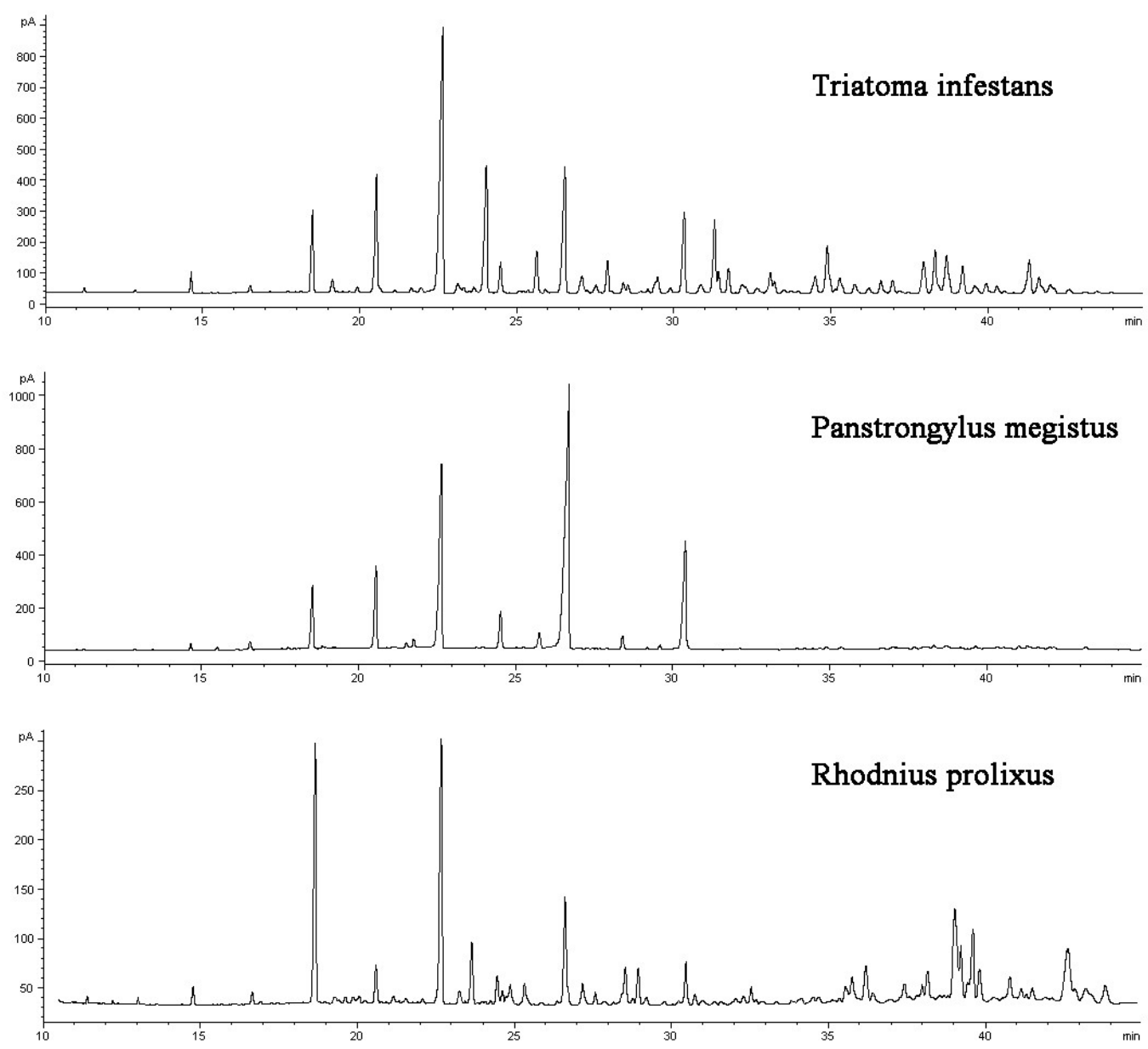

En las especies del género Triatoma sp. estudiadas hasta el momento, así como en las del género Rhodnius sp., los hidrocarburos cuticulares consisten en mezclas complejas de cadenas lineales y metil ramificadas, saturadas, de 18 a más de 40 átomos de carbono y con 1 a 3 ramificaciones metilo; además, únicamente en el género Rhodnius sp. se han encontrado hidrocarburos con 4 ramificaciones metilo sobre la cadena principal (Juárez y Brenner 1987, Juárez y Blomquist 1993, Juárez et al. 2001, Juárez y Calderón Fernández 2007). El patrón de hidrocarburos de Triatoma infestans, T. mazzottii Usinger 1941 y Rhodnius prolixus identificados mediante cromatografía gaseosa capilar acoplada a espectrometría de masas se detalla en la tabla 2.

\section{$\underline{n \text {-Alcanos }}$}

Dentro de esta fracción predominan de manera característica los componentes de cadena impar, de 25 a 33 carbonos; los hidrocarburos de cadena par de 18 a 32 carbonos, así como los de cadena impar de 21 a 23 carbonos constituyen componentes minoritarios. De manera característica el $n \mathrm{C} 33$ tiene mayor proporción que el $n \mathrm{C} 31$ durante los estadios 
ninfales mientras que el $n \mathrm{C} 29$ es mayor o igual que el $n \mathrm{C} 31$ durante el estadio de adulto, teniendo el nC33 una baja proporción (Juárez y Brenner 1985, Juárez y Blomquist 1993, Juárez et al. 2001). En T. infestans (Juárez et al. 1985) comprenden cerca del $40 \%$ de los hidrocarburos cuticulares, encontrándose una proporción algo mayor en $T$. pallidipennis Stal 1872 (aprox. 45 \%) (Juárez y Brenner 1987)

\section{$\underline{n \text {-Alquenos }}$}

La mayoría de las especies estudiadas carecen de hidrocarburos insaturados, sin embargo se han encontrado a niveles de traza en $T$. mazzottii, detectándose un hidrocarburo insaturado lineal de 33 carbonos y otro no identificado (Juárez y Blomquist 1993)

\section{$\underline{\text { Alcanos ramificados }}$}

La composición de hidrocarburos ramificados en triatominos consiste en una mezcla compleja de mono, di, tri y tetrametilalcanos, aunque estos últimos sólo se han detectado hasta el momento en el género Rhodnius sp. (Juárez y Brenner 1987, Juárez y Blomquist 1993, Juárez et al. 2001, Juárez et al. 2002).

\section{$\underline{\text { Monometilalcanos }}$}

Se encuentran cadenas con ramificaciones en posiciones pares e impares de la cadena principal, donde comunmente las cadenas pares presentan la ramificación en posición par, mientras que las impares la presentan mayoritariamente en posición impar y escasamente en par. T. infestans exhibe un patrón diverso de monometilalcanos con ramificaciones en posición terminal (3-) insertadas en cadenas de 25 a 31 carbonos y de manera característica una ramificación en posición subterminal (4-) en una serie homóloga de cadenas pares de 26 a 36 carbonos; como ramificaciones internas se destacan las insertadas en posiciones 11- y 13 de cadenas impares de 27 a 39 carbonos. En T. mazzottii predominan los monometilalcanos internos con inserciones de grupos metilo en posiciones 11- y 13- de series homólogas de cadenas de 27 hasta 41 carbonos y en posiciones impares 15- a 21- de hidrocarburos de muy larga cadena de 39 hasta 43 carbonos; esta especie presenta de manera distintiva una ramificación en posición 12- de cadenas pares de 32 a 40 carbonos. $R$. prolixus se caracteriza por carecer de ramificaciones ubicadas en posición par, así como por presentar un patrón amplio de posiciones de inserción, desde terminales (3-) hasta internas (21-), con cadenas que van desde 27 a 41 carbonos y con series homólogas de 27 a 39 carbonos que presentan ramificaciones en posiciones 11-, 13- y 15- de la cadena. 


\section{Dimetilalcanos}

El patrón de dimetilalcanos de triatominos está formado por compuestos con ramificaciones terminales e internas de la cadena principal y donde de manera predominante las mismas están separadas por 3 o 9 grupos metileno. T. infestans presenta los isómeros terminales y subterminales 3,7-, 3,9- y 4,8- en cadenas de 29 a 31 carbonos (Juárez, no publicado), mientras que los dimetilalcanos internos consisten en isómeros donde la primer ramificación está en posición 11-, 13- o 15- en cadenas de 27 a 39 carbonos y donde no se encuentran largas series homólogas (tabla 2) (figura 11B). En T. mazzottii se detectan dimetilalcanos subterminales del hidrocarburo C37 (5,13- y 5,15-), series homólogas impares de 25 a 33 átomos de carbono, de isómeros con la primer ramificación en posición 9- y 11- y series homólogas pares de 34 a 40 carbonos con ramificaciones en posiciones 12,20- y 12,22-; además de presentar alcanos de larga cadena (39 a 43 carbonos) con ramificaciones 11,21-, 13,21- y 13,23-, esta especie exhibe de forma excepcional dimetilalcanos con 1 interrupción metileno, como el isómero 11,13- en cadenas de 25, 27 y 33 carbonos (tabla 2)(figura 11A). En $R$. prolixus solo se detectaron cuatro dimetilalcanos de 35, 37 y 41 carbonos con ramificaciones en las posiciones 5,15-, 5,17- y 7,11- de la cadena lineal (figura 12a).

\section{$\underline{\text { Trimetilalcanos }}$}

El contenido de trimetilalcanos es escaso en los miembros del género Triatoma sp. con 3 y 4 de estos compuestos en $T$. infestans y T. mazzottii respectivamente, mientras que en Rhodnius sp. se pueden contar mas de 20 trimetilalcanos (Tabla 2). Asimismo las cadenas pares tienen sus ramificaciones en posiciones pares mientras que las impares las tienen en posición impar. T. infestans carece de cadenas pares y presenta cadenas de 35 a 39 carbonos con inserciones en las posiciones 13,17,21- y 13,17,23; mientras que T. mazzottii presenta una cadena par (el 12,16,22-C38) y otros tres trimetilalcanos de 33, 39 y 41 carbonos con ramificaciones en la posiciones 11,15,21- y 11,15,23 de la cadena principal (figura 11C). $R$. prolixus presenta una profusa cantidad de trimetilalcanos con cadenas de 29 a 41 carbonos e isómeros cuya primer ramificación van desde la posición 5- a la 11- en la cadena lineal (ejemplos son los isómeros 5,9,13-, 7,11,19-, 8,12,20-, 9,13,19- y 11,15,19-) (tabla 2)(figura $12 b)$.

\section{$\underline{\text { Tetrametilalcanos }}$}

Estos hidrocarburos sólo se han encontrado en el género Rhodnius sp. en cadenas impares de 29 a 41 carbonos y pares de 36 a 40 carbonos y con ramificaciones separadas por 
3 hasta 15 grupos metileno. Las primeras ramificaciones se ubican en posiciones 3-, 5-, 6-, 7y 8- de la cadena mientras que las últimas lo hacen en posciones terminales de 18- hasta 30(ejemplos 3,9,13,19-tetrame-C37, 6,10,14,30-tetrame-C40) (figura 12c).

Tabla 2. Hidrocarburos cuticulares identificados en Triatoma infestans, T. mazzottii y Rhodnius prolixus.

\begin{tabular}{|c|c|c|c|}
\hline & T. infestans & T. mazzottii & R. prolixus \\
\hline \multicolumn{4}{|l|}{ Hidrocarburos lineales } \\
\hline \multicolumn{4}{|l|}{ cadena lineal par } \\
\hline $\mathrm{C} 18$ & + & - & + \\
\hline $\mathrm{C} 20$ a C28 & + & + & + \\
\hline C30 y C32 & + & + & - \\
\hline \multicolumn{4}{|l|}{ cadena lineal impar } \\
\hline C19 & + & - & + \\
\hline $\mathrm{C} 21$ a C33 & + & + & + \\
\hline
\end{tabular}

Hidrocarburos ramificados

Monometil terminales

4-MeC26 a C36

3-MeC25 a C31

5-MeC29 y C31

Monometil internos

Cadena lineal par

11- y 13-MeC30

12- a 14-MeC32 a C40

Cadena lineal impar

7-MeC27 y C29

9-MeC29

11-MeC27 a C41

$13-\mathrm{MeC} 27$ a $\mathrm{C} 41$

15-MeC29 a C41

17- 19- y 21-MeC39 a C43

Dimetil terminales e internos

Cadena lineal par

3 interrupciones $\mathrm{CH} 2$

4,8- y 13,17-DiMeC30 a C32

7 y 9 interrupciones $\mathrm{CH} 2$

9,19- 11,21- 12,20- y 12,22-DiMeC30 a 40 
Continuación tabla 2.

Cadena lineal impar

1 interrupción $\mathrm{CH} 2$

11,13-DiMeC25 a C33

3 interrupciones $\mathrm{CH} 2$

3,7- 9,13- y 15,19-DiMeC27 a C33

11,15- y 13,17-DiMeC25 a C33

7,11-DiMeC35

$5 \mathrm{a} \geq 9$ interrupciones $\mathrm{CH} 2$

3,9-DiMeC29 y 13,19-DiMeC35

5,13- 5,15- y 5,17-DiMeC35 a C41

9,19-DiMeC29 y C31

11,21- 11,23- 13,21- 13,23-DiMeC31 a C43

13,21DiMeC33 and C37

15,25-DiMeC39

\section{Trimetil internos}

Cadena lineal par

3-5 a 3-15 y 5-3 interrupciones $\mathrm{CH} 2$

8,x,y-TriMeC36 a C40

12,x,y-TriMeC38

Cadena lineal impar

3-3 a 3-13 y 5-3 interrupciones $\mathrm{CH} 2$

5,x,y- 7,x,y- 9,x,y-TriMeC29 a C41

11-x-y-TriMeC29 a C41

13,x,y-TriMeC35 a C39

\section{Tetrametil terminales e internos}

Cadena lineal par

3-x-y y 7-x-y interrupciones $\mathrm{CH} 2$

6,x,y,z- y 8,x,y,z-TetraMeC36 a C40

Cadena lineal impar

3-x-y y 5-x-y interrupciones $\mathrm{CH} 2$

3,x,y,z- 5,x,y,z- y 7,x,y,z-TetraMeC29 a C41

$x-y-z$ : las letras hacen referencia a la cantidad de grupos metileno entre ramificaciones

$\mathrm{x}, \mathrm{y}, \mathrm{z}$ : las letras hacen referencia a la posición de las ramificaciones en la cadena lineal

datos según Juárez y Blomquist 1993 y Juárez et al. 2001

\section{7. Análisis cuantitativo y cualitativo de los hidrocarburos. Cromatografía Gaseosa} Capilar (CGC) y CGC acoplada a Espectrometría de Masa (CGC-MS).

Como se ha mencionado antes, los hidrocarburos de insectos se presentan como mezclas complejas, por lo que la identificación de los distintos compuestos se basa en la separación de los mismos mediante cromatografía gaseosa capilar (CGC) y la determinación de sus estructuras mediante espectrometría de masa (MS). 
La cuantificación de hidrocarburos o de cualquier compuesto mediante CGC se basa en que el área de los picos registrados en el cromatograma es proporcional a la masa del compuesto inyectado; de esta manera, el procedimiento general de un análisis cuantitativo por CGC involucra la obtención del cromatograma de la muestra, la medida del área de los picos de interés y el cálculo de la masa correspondiente a cada uno de los picos. Este cálculo se hace empleando una curva de calibración basada en la inyección de un estándar de concentración conocida. El cálculo posterior del área obtenida a partir del estándar permite correlacionar valores de área con valores de masa. Una forma alternativa de cuantificación es el referirse al porcentaje del área de un pico en función del área total de todos los picos contenidos en el cromatograma; la comparación de los porcentajes entre distintas muestras permite establecer diferencias entre ellas.

El análisis cualitativo hace referencia a la determinación de la identidad química de los compuestos contenidos en una muestra; el método más difundido es la CGC acoplada a MS (CGC-MS). En la MS de impacto electrónico, los hidrocarburos de una muestra son fragmentados e ionizados por una corriente de electrones generando un patrón de fragmentos que es específico de cada molécula; es característico de esa fragmentación la formación de grupos de iones cada catorce unidades de masa, que corresponden al clivaje de las uniones carbono-carbono de la cadena principal (figura 9). El ion al final de la escala de masas es el ion molecular $\left(\mathrm{M}^{+}\right)$, que corresponde al hidrocarburo sin fragmentar y mediante el cual es posible determinar su peso molecular. La presencia del $\mathrm{M}^{+}$permite una rápida identificación del hidrocarburo, sin embargo, es difícilmente detectable en los hidrocarburos de cadena muy larga y en los ramificados.

Figura 9. Espectro de iones característico de la fragmentación de un alcano.

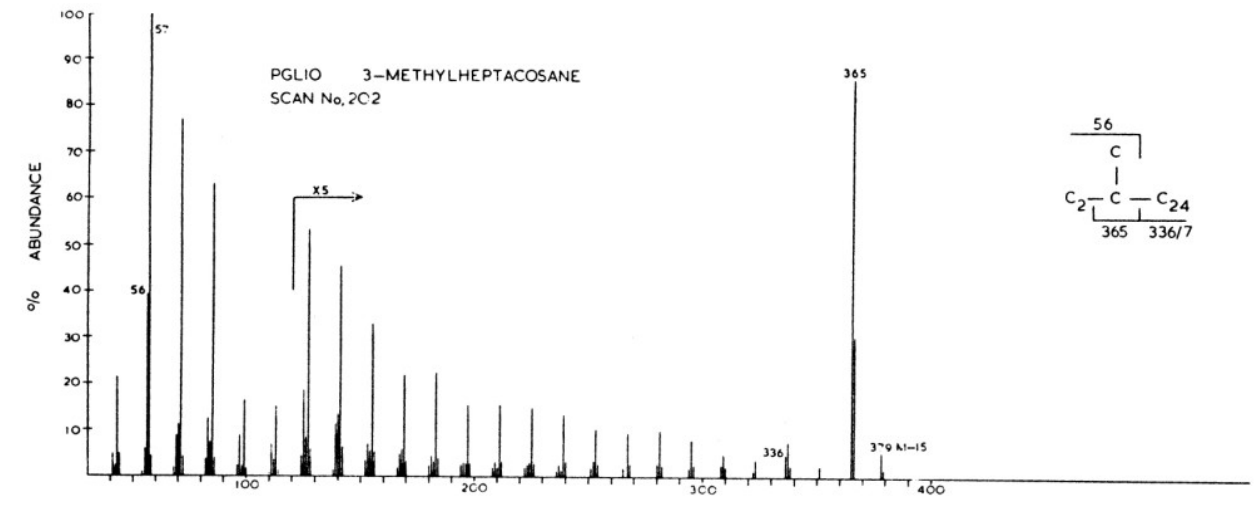

3-metilheptacosano, un monometilalcano terminal. Tomado de Lockey 1988

Los metilalcanos se fragmentan preferencialmente a ambos lados de la ramificación, y muestran un incremento en la intensidad del ion M-15 como resultado de la ruptura al nivel de 
la ramificación metilo. En los monometilalcanos terminales, la liberación de un grupo isopropilo en la fragmentación de los 2-metilalcanos y de un grupo etilo en la de los 3metilalcanos, da como resultado un incremento del ion M-43 y M-29 respectivamente, que resultan de una intensidad similar al del M-15. En los monometilalcanos internos los M-43, M-29 y M-15 forman un grupo de iones de intensidad creciente el cuál ayuda a determinar la ubicación del $\mathrm{M}^{+}$y por lo tanto contribuye a la identificación del compuesto (figura 10). Una característica de la fragmentación de los monometilalcanos internos, es la formación de un fragmento iónico de masa par debida a la pérdida de un átomo de hidrógeno tanto en el fragmento que porta la ramificación como en el fragmento lineal (McCarthy et al. 1968), de tal manera que en el espectro aparecen una serie de dobletes iónicos; por ejemplo, la ruptura en el lado interno de la ramificación del 9-metil-C29, generará un par de iones de masa 140 y 141 (donde el 140 es resultado de la pérdida de un hidrógeno del 141) junto al par 280 y 281, mientras que la ruptura en el lado externo generará los pares 308 y 309 junto al par 112 y 113 (figura 10C). La formación del ion de masa par queda suprimida cuando en la cadena se encuentra más de una ramificación (McCarthy et al. 1968, Blomquist et al. 1987).

Figura 10. Ejemplos idealizados de la fragmentación de hidrocarburos mediante espectrometría de masa de impacto electrónico.

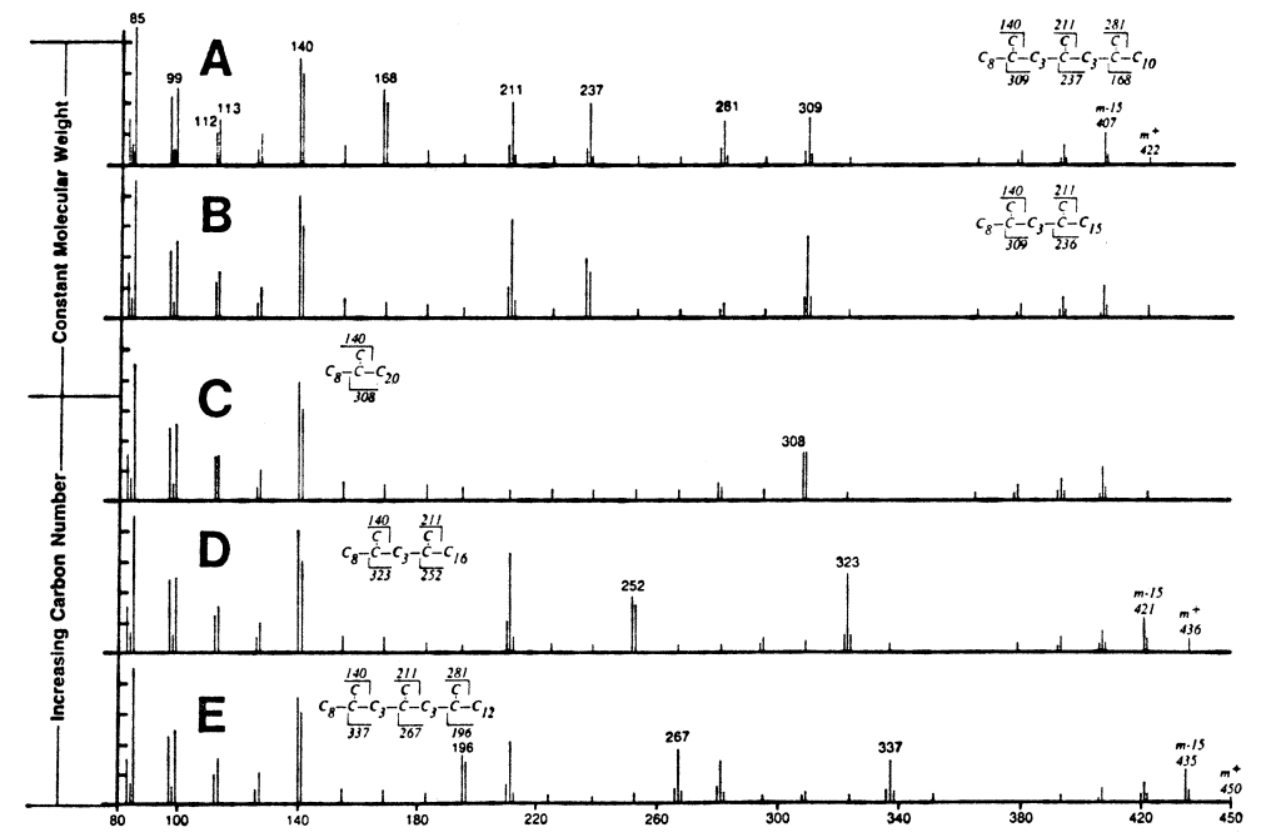

A y E: trimetilalcanos, B y D: dimetilalcanos, C: monometilalcano. Tomada de Blomquist et al. 1987

La figura 11 muestra tres espectros de masa pertenecientes a hidrocarburos epicuticulares de T. infestans y T. mazzottii (Juárez y Blomquist 1993) mientras que la figura 
12 muestra tres espectros pertenecientes a R. prolixus (Juárez et al. 2001). En el punto I. 6. se comentan los hidrocarburos encontrados en estas especies.

Figura 11. Espectros de masa de hidrocarburos cuticulares de T. infestans y T. mazzottii.
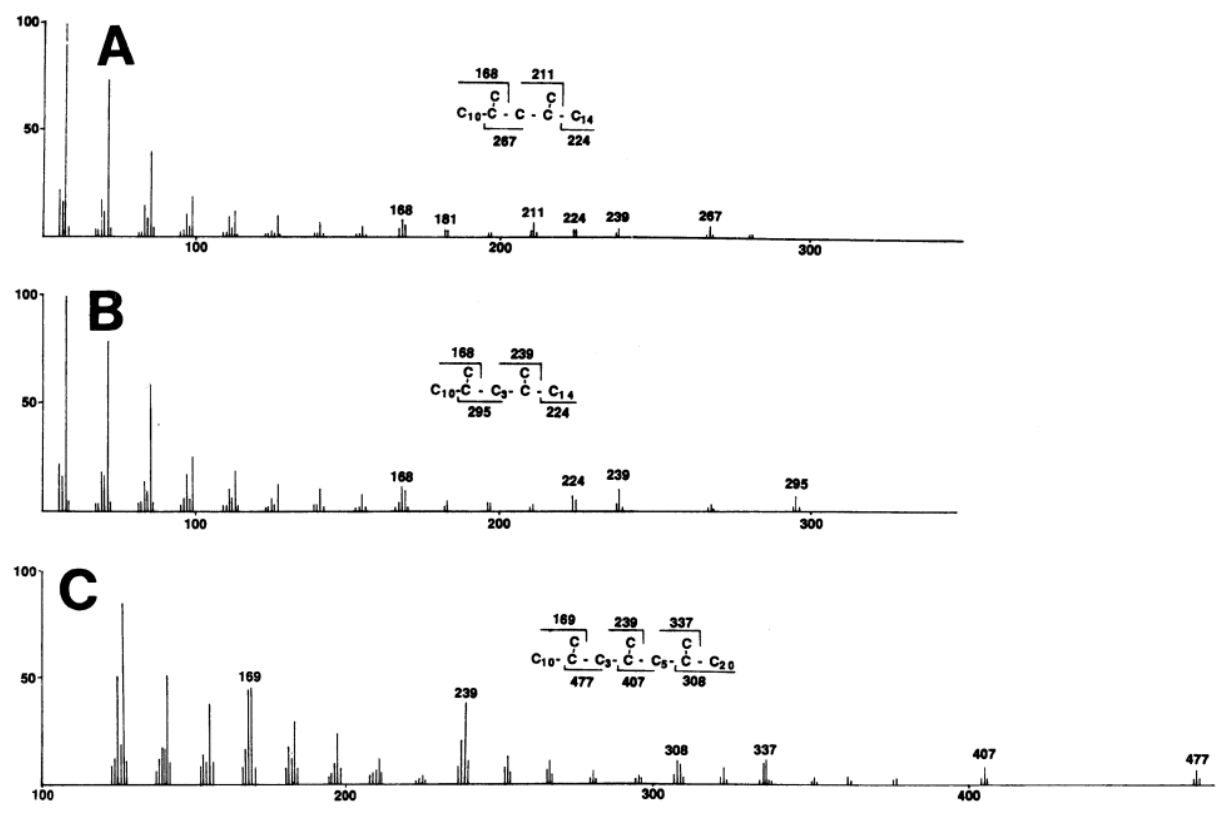

A) 11,13-dimetilheptacosano; B) 11,15-dimetilnonacosano; C) 11,15,21-trimetilhentriacontano. Tomada de Juárez y Blomquist 1993

Figura 12. Espectros de masa de hidrocarburos cuticulares de R. prolixus
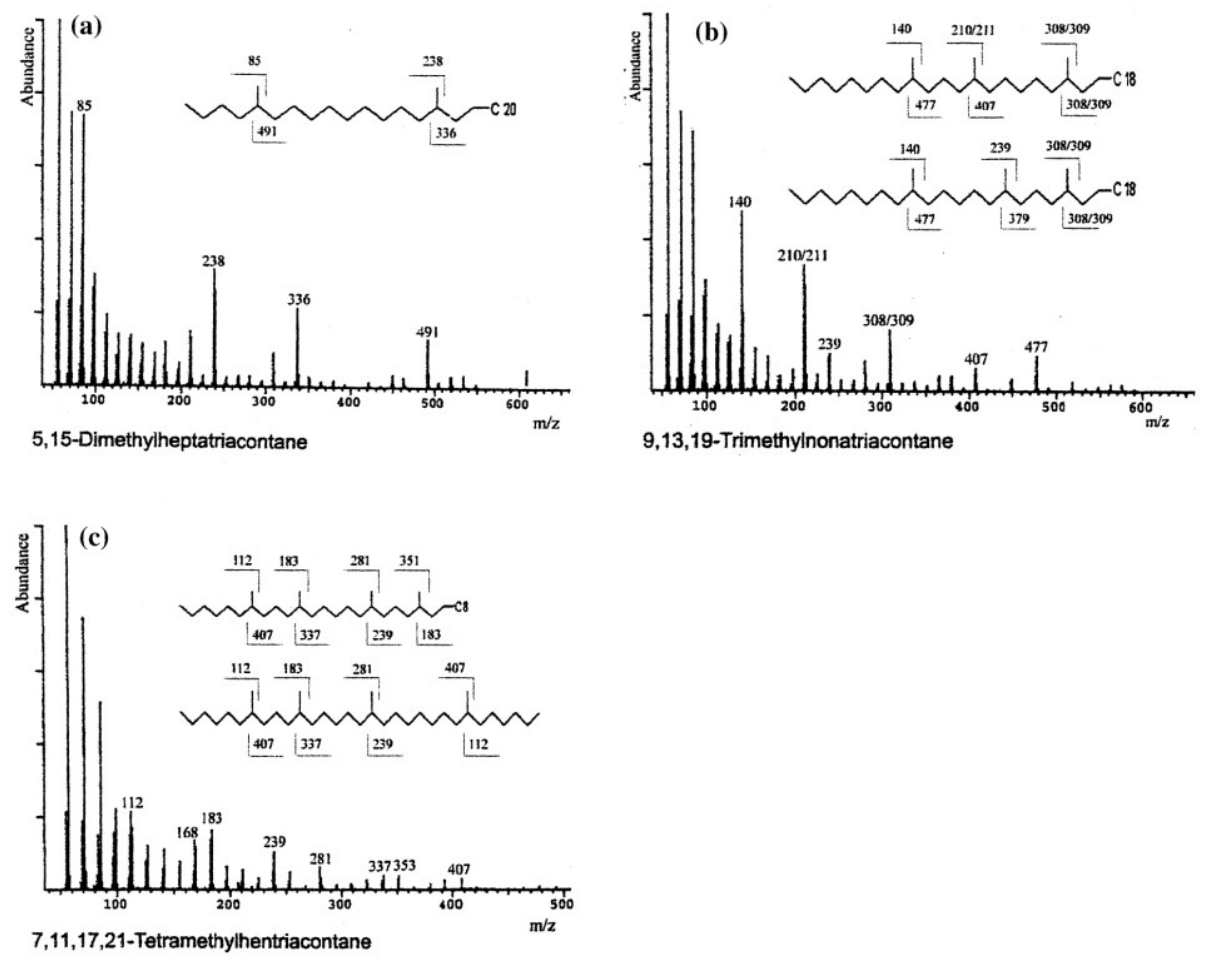

a) 5,15-dimetilheptatriacontano; b) 9,13,19-trimetilnonatriacontano; c) 7,11,17,21-tetrametilhentriacontano. Tomada de Juárez et al. 2001 
Las bases de datos disponibles para interpretar los espectros de masa obtenidos, contienen escasos hidrocarburos de la longitud de cadena y de los tipos que se encuentran en insectos (Carlson et al. 1998). No hay disponibles bibliotecas de espectros de HC ramificados de insectos, y en la mayoría de los casos no es posible visualizar el $\mathrm{M}^{+}$, por lo cual es necesario interpretar cada espectro individual. Una medida adicional y de gran utilidad, que provee una aproximación a la estructura del compuesto y que da una identificación tentativa del mismo es el Indice de Kovats (KI) o Indice de Retención (Kovats 1965), el cual da una medida de la cantidad de carbonos en la cadena y contribuye a determinar la identidad del compuesto. En el caso de los hidrocarburos lineales saturados los KIs tienen valores de centenas enteros (ej: $n \mathrm{C} 23=\mathrm{KI} 2300, n \mathrm{C} 29=\mathrm{KI} 2900, n \mathrm{C} 32=\mathrm{KI} 3200$ ). En los monometilalcanos, la presencia de una ramificación metilo en la cadena alquílica suele acortar su tiempo de retención comparado al de una cadena normal con el mismo número de carbonos (figura 13). La posición de la ramificación determina la magnitud de la reducción, de tal manera que los isómeros con la ramificación en el centro tienen los tiempos de retención más cortos (aproximadamente 70 unidades menos), mientras que a medida que la ramificación se ubica cerca del extremo de la cadena, el valor de KI se aproxima al del correspondiente $n$ alcano con el mismo número de carbonos (Blomquist et al. 1987, Lockey 1988, Carlson et al. 1998). En los dimetilalcanos, la presencia de una ramificación metilo extra, aproximadamente duplica el efecto sobre el tiempo de retención por lo que el KI es entre 90 a 160 unidades menor que el correspondiente al $n$-alcano con el mismo número de carbonos; el menor desplazamiento del KI se obtiene cuando una de las ramificaciones se encuentra en posición terminal o subterminal de la cadena (ej: 3,X-diMe-C33 KI 3403 a 3409, 5,X-diMe-C33 KI 3379 a 3382, 13,X-diMe-C33 KI 3340 a 3362) (Blomquist et al. 1987, Lockey 1988, Carlson et al. 1998). Del mismo modo, en los trimetil y tetrametilalcanos la presencia de ramificaciones adicionales, triplica y cuadruplica respectivamente el efecto sobre el tiempo de retención y el KI disminuye 160 a 220 unidades para los trimetilalcanos y 240 a 290 unidades para los tetrametilalcanos, respecto a un $n$-alcano de la misma cantidad de carbonos (figura 13) (Blomquist et al. 1987, Lockey 1988, Carlson et al. 1998). Estas desviaciones dependen a su vez de la longitud de cadena de la porción lineal. 
Figura 13. Valores de Indices de Kovats calculados para un hidrocarburo con una a cuatro ramificaciones metilo insertadas en una cadena lineal de 33 átomos de carbono.

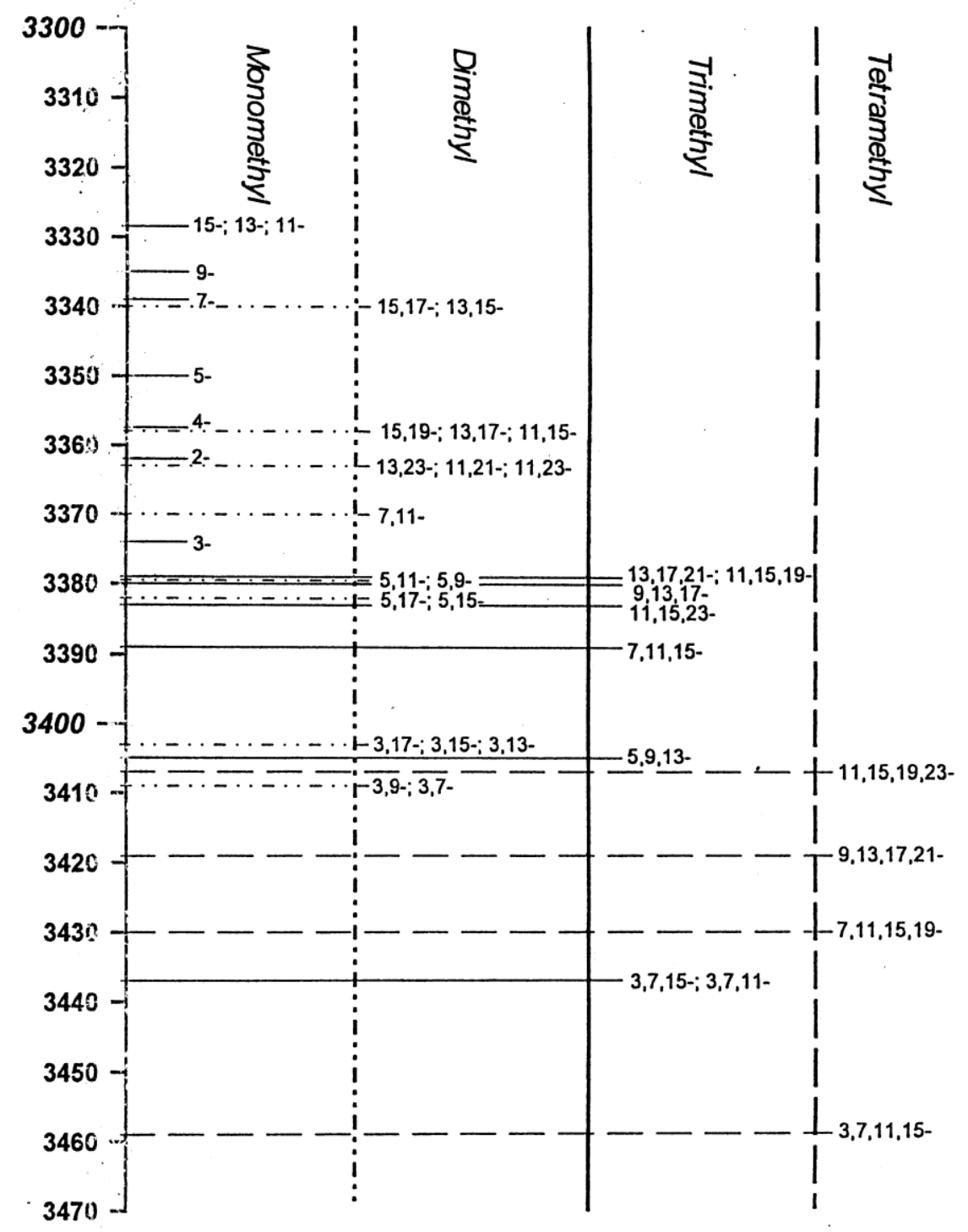

Tomada de Carlson et al. 1998

En diversas oportunidades y aún en el caso de una buena resolución cromatográfica, el valor de KI de un pico no permite asociarlo de manera unívoca a un hidrocarburo, ya que en ese pico pueden coeluir varios compuestos simultáneamente. Por ejemplo, a partir de la figura 13 se puede apreciar que los monometilalcanos 11-, 13- y 15-Me-C33 coeluyen juntos, y así para los dimetilalcanos 5,11- y 5,9-diMe-C33, y los trimetilalcanos 13,17,21- y 11,15,19triMe-C33. Más aún, en un mismo pico pueden coeluir mono y dimetilalcanos (4-Me-C33 junto a 15,19-, 13,17- y 11,15-diMe-C33) o dimetil y trimetilalcanos juntos (5,11- y 5,9diMe-C33 junto a 13,17,21-, 11,15,19- y 9,13,17-triMe-C33) (Carlson et al. 1998).

Una identificación confiable de la estructura de los hidrocarburos resulta de la interpretación de los espectros de masa junto con el análisis de los valores de índices de retención y la factibilidad biosintética de las estructuras propuestas (Blomquist et al. 1987). 


\section{8. Nuevas técnicas y marcadores de aplicación en taxonomía.}

Con el objetivo de complementar la información obtenida a partir de los análisis taxonómicos tradicionales o resolver cuestiones que éstos no pueden dilucidar, se han desarrollado en la segunda mitad del siglo pasado, un conjunto de técnicas basadas en caracteres bioquímicos, moleculares y morfométricos. Colectivamente estas técnicas y caracteres tratan de capturar la variación genética en los especímenes, independizándose de la producida por el ambiente y por fenómenos alométricos.

Dentro de los marcadores bioquímicos se puede mencionar el estudio del polimorfismo en el patrón de isoenzimas. Estas son extraídas de distintos órganos dependiendo del grupo de especímenes en estudio y su variación aporta información sobre las relaciones de similitud entre ellos. Se han empleado en el análisis taxonómico de bacterias (Jana 1994), hongos (Carder 1989, Sing et al. 1996, Ramos et al. 1999), protozoos (Goncalves de Lima et al. 1979, Tibayrenc y Miles 1983, Rioux et al. 1990), plantas (Proctor et al. 1989, Jaaska 1994, Altube et al. 2001) y animales (Petersen y Russell 1971, Ostman 1982, Verdyck 1999).

La morfometría comprende un conjunto de métodos para la descripción cuantitativa, el análisis y la interpretación biológica de la forma y sus variaciones (Rohlf 1990). Se ha empleado en el estudio de la diversidad de protistas (Bellier y De Wever 1994), plantas (Shipunov y Bateman 2005) y animales de diversos ordenes incluido el hombre (Ferrario et al. 1993, Capanna et al. 1996, Adams y Funk 1997, Drotz et al. 2001).

Los caracteres moleculares se han utilizado extensivamente para estimar la variabilidad intraespecífica en organismos, estudiar las relaciones entre taxa a varios niveles jerárquicos y estimar filogenias. Entre los más usados se pueden mencionar los análisis de secuencias de: DNA mitocondrial (Mamuris et al. 1999, Pastorini et al 2003, Zaccara et al. 2004), rDNA-ITS nuclear (espaciador interno de transcripción del DNA ribosómico) (Paul 2000, Huss et al. 2002, Jorgensen et al. 2003), LSU rDNA (subunidad grande del DNA ribosómico) (Leclerc et al. 2000, Paulin-Mahady et al. 2002, Sonnenberg et al. 2007) y RAPD (amplificación aleatoria de fragmentos polimórficos de DNA)(Pacak et al. 1998, Mamuris et al. 1999, Jorgensen et al. 2003).

En triatominos, se han empleado las secuencias de DNA mitocondrial en el estudio de las relaciones filogenéticas entre varias especies de los géneros Triatoma sp., Rhodnius sp. y Panstrongylus sp. (Lyman et al. 1999, Sainz et al. 2004) y dentro del género Triatoma sp. (Garcia y Powell 1998); en el análisis de la variabilidad intraespecífica de T. infestans (y su relación con Triatoma melanosoma Martínez et al. 1987 y Triatoma brasiliensis Neiva 1911 (Monteiro et al. 1999) y de T. brasiliensis (Monteiro et al 2004). Se han empleado análisis 
morfométricos basados en medidas de la cabeza, alas y patrones de sensilas antenales para estudiar la filogenia de la tribu Rhodniini (Dujardin et al. 1999), la variabilidad intraespecífica de T. infestans (Casini et al. 1995, Catala y Dujardin 2001) y de Pastrongylus rufotuberculatus Champion 1899 (Dujardin et al. 1998a), y el origen de los triatominos europeos y asiáticos (Patterson et al. 2001). Los análisis isoenzimáticos se utilizaron en el estudio del proceso de domesticación de T. infestans (Dujardin et al. 1987) y de su variabilidad (Dujardin et al. 1998b, Acosta el al. 2002), la variabilidad intraespecífica de $T$. brasiliensis (Costa et al. 1997) y de la filogenia de la tribu Rhodniini (Dujardin et al. 1999) y el complejo infestans (Pereira et al. 1996). Los análisis basados en caracteres moleculares se han aplicado tanto a estudios a pequeña escala (de variabilidad entre poblacionales, especies y géneros) (Marcilla et al. 2002, Breniere et al. 2003, Martínez et al. 2006, Dos Santos et al. 2007) como en la inferencia de las relaciones filogenéticas de toda la subfamilia (Bargues et al. 2000, Marcilla et al. 2001, Hypsa et al. 2002, Galvao 2003, Jurberg 2003, Tartarotti y Ceron 2005).

\section{9. Aplicación del análisis de hidrocarburos cuticulares en taxonomía de insectos y plantas}

Recién a partir de los últimos 30 años, el patrón de hidrocarburos cuticulares ha sido empleado como marcador taxonómico para la identificación de géneros y especies en diversos órdenes de insectos, especialmente en especies de importancia médica y económica. Las variaciones cualitativas y cuantitativas en las distintas clases de hidrocarburos cuticulares no sólo han permitido identificar especies crípticas, indistinguibles por métodos morfológicos tradicionales, han servido también para estudiar las diferencias entre poblaciones y establecer la variabilidad genética intraespecífica. Tal como las características morfológicas y la estructura de las enzimas metabólicas, el patrón de hidrocarburos está genéticamente fijado y representa un fenotipo especie específico (Kaib et al. 1991, Lockey 1991, Etges y Jackson 2001). La presencia, ausencia o abundancia relativa de los distintos hidrocarburos está relacionada a la ausencia, presencia o cinética de las enzimas biosintéticas (Page et al. 1997). Mediante una serie de cruzamientos experimentales, Coyne (1996) pudo determinar que la variación de los hidrocarburos cuticulares responsables del dimorfismo sexual entre especies del grupo Drosophila melanogaster (D. melanogaster Meigen 1830 y D. simulans Sturtevant 1919), está determinada por una serie de loci en el cromosoma 3. En estudios con mutantes realizados en plantas de los géneros Arabidopsis sp. Heynh y Zea sp. Linnaeus, se ha podido observar que un gran número de genes están involucrados en la síntesis de los hidrocarburos cuticulares, habiéndose aislado cuatro de estos (Lemieux 1996). 
Dentro de las especies de importancia médica, Carlson y Service (1979 y 1980) fueron los primeros en separar dos especies del complejo Anopheles gambiae Giles mientras que Milligan y col. (1986) separaron tres especies del complejo A. culicifacies Giles 1901. Veintiseis especies de la mosca tse-tse (Glossina sp.) pudieron ser diferenciadas por Carlson y col. (1993), además de seis especies de la mosca negra africana Simulium damnosum Theobald 1903 (Carlson y Walsh 1981, Phillips et al. 1985) y ocho especies del género Tabanus sp. Linnaeus 1758 (Díptera: Tabanidae) (Hoppe et al. 1990). Dentro de los insectos de importancia económica se han separado las abejas europeas de las africanizadas (Carlson y Bolten 1984) y se han estudiado especies de importancia agronómica tales como plagas agrícolas (Hooper et al. 1996) y diversas especies de coleópteros que afectan la producción forestal (Page et al. 1990a,b; 1997). Las variaciones fenotípicas de los hidrocarburos cuticulares se han analizado extensamente en diversas especies de termitas, no sólo con el objetivo de esclarecer problemas taxonómicos de los distintos grupos, sino también para estudiar el rol que estos compuestos pueden tener en la diferenciación entre castas y el reconocimiento de conespecíficos y especies territorialmente vecinas (Haverty et al. 1988, 1990a,b; Howard et al. 1978, 1982; Kaib et al. 1991, 2002, 2004). Estos componentes cuticulares han servido además en la separación de especies de cucarachas (Carlson y Brenner 1988, Brown et al. 2000) y de distintas grupos del género Drosophila sp. (Jallon y David 1987, Etges y Jackson 2001).

Más recientemente, la utilización del patrón de hidrocarburos cuticulares para estudios taxonómicos, se ha hecho extensiva también a las plantas, aplicándose este análisis, a diversos grupos de plantas vasculares (Gülz 1994, Maffei 1996, Mimura et al. 1998, Barthlott et al 2003, Medina et al. 2006).

En triatominos, Juárez y Brenner (1986) analizaron la composición de hidrocarburos de machos y hembras de siete especies, seis de América del Sur (Triatoma infestans, T. rubrovaria Blanchard 1843, T. delpontei Romaña y Abalos 1947, T. vitticeps Stal 1859, T. sordida y $T$. guasayana) y una de América del Norte (T. pallidipennis). Se encontraron diferencias cuantitativas en los perfiles de hidrocarburos que permitían distinguir claramente a estas especies y en algunos casos diferenciar los sexos ( $T$. delpontei y $T$. sordida), sin embargo las limitaciones técnicas locales no permitieron separar todos los componentes. Posteriormente se analizaron mediante GCG-MS los hidrocarburos cuticulares de T. infestans, T. mazzottii (Juárez y Blomquist 1993) y Rhodnius prolixus (Juárez et al 2001) encontrándose que las diferencias entre las especies no solo están dadas por las variaciones cuantitativas de los componentes comunes sino también por las diferencias isoméricas en las cadenas metil ramificadas. Adicionalmente en el género Rhodnius sp. se hallaron cadenas tetrametil 
ramificadas ausentes en otros géneros de la tribu Triatomini (Triatoma sp. y Panstrongylus sp.). Esta diferencia junto a otras variaciones en las proporciones de las cadenas metil ramificadas contribuyó a la discriminación de los principales géneros de la subfamilia y a una neta diferenciación de la tribu Rhodniini (Juárez et al. 2000, Juárez y Calderón Fernández 2007).

\section{10. Métodos de ordenación y clasificación en el estudio de la sistemática de insectos}

Los métodos de ordenación y clasificación son técnicas estadísticas multivariadas que representan los patrones de variación atribuibles a múltiples caracteres en un número reducido de dimensiones. Permiten asimismo determinar el aporte de cada carácter a la variación total de los individuos en estudio (Foottit y Sorensen 1992). Los métodos de ordenación se pueden agrupar de manera general en dos categorías: aquellos que analizan muestras individuales, como el Análisis de Componentes Principales (PCA) y aquellos que analizan dos o más muestras que pueden estar agrupadas, tal como el Análisis Discriminante (DA)

El PCA ha sido usado exhaustivamente como medio de exploración de los datos y de reducción de variables. Esta técnica no asume una separación a-priori de los especímenes en grupos separados, por lo que el análisis se concentra en las relaciones entre las variables y entre los especímenes en estudio. Las variables (caracteres) originales son transformadas en un nuevo set de variables, los componentes principales, que constituyen combinaciones lineales de las variables originales. El cálculo de los componentes principales implica que todas las variables contribuyen en mayor o menor medida a cada componente, y que la variación total de la muestra es acumulada en mayor proporción en el primer componente y en orden decreciente en los siguientes. De esta manera un número reducido de componentes acumulan casi toda la variabilidad de la muestra.

El DA, en contraste con el PCA, implica un agrupamiento de los especímenes previo al análisis; estos grupos previos pueden representar entidades taxonómicas definidas en base a algún criterio, o individuos que comparten alguna característica en común. Si bien el DA es una extensión de PCA tiene diferencias fundamentales con este último, de cálculo (no detalladas aquí) y en cuanto a los resultados (Pimentel 1992). El principal objetivo del DA es calcular funciones lineales basadas en las variables (caracteres) originales medidas en las muestras, en este sentido se asemeja al PCA. Sin embargo, este cálculo conlleva la partición de la variación total en la compartida entre los grupos y la de cada grupo y subsiguientemente, la maximización de la varianza entre grupos respecto a la varianza dentro de los grupos. Esto implica un análisis de la variación mas allá de la común a los grupos o poblaciones (variabilidad dentro de los grupos), mientras que el PCA analiza los patrones de variación 
total de los especímenes. De esta manera, en el DA se obtienen nuevas variables, las funciones discriminantes, que permiten obtener la máxima separación entre los grupos en estudio. Asimismo, a partir de los coeficientes estandarizados de la funciones discriminantes o de los valores de correlación entre éstas y las variables originales, se pueden deducir las variables originales que más contribuyen a la discriminación entre los grupos.

Los métodos de ordenación aplicados a diversos caracteres fenotípicos han sido profusamente usados en el estudio de especies y poblaciones así como en la inferencia de relaciones filogenéticas entre taxa. Comúnmente los cladogramas están identificados con hipótesis filogenéticas mientras que a los métodos de ordenación se los asocia a hipótesis de relaciones fenéticas entre las unidades de estudio. En este sentido se considera que los cladogramas representan relaciones temporales mientras que los dendrogramas derivados de la ordenación representan relaciones espaciales. Sin embargo, los métodos de ordenación (y especialmente el DA) podrían implicar además una aproximación filogenética a las relaciones entre los especímenes. La partición de la variación de los especímenes en múltiples dimensiones o en variación compartida versus divergente (como la obtenida a partir del DA), podrían llegar a mostrar la dirección de cambio de los caracteres derivados, más que simplemente mostrar patrones de similitud global (Foottit y Sorensen 1992).

La aplicación conjunta del DA y el PCA ha sido de utilidad en numersos trabajos destinados a esclarecer y distinguir relaciones entre taxas crípticos. Permitió la discriminación de especies de áfidos del género Cinara sp. Curtis 1835 junto con la selección de los caracteres que mejor contribuyen a esa discriminación (Foottit 1992). El análisis de diversas características morfológicas permitió reforzar la idea de que dos variantes del odonato Tramea binotata Rambur 1842 caracterizadas por la diferencia de color, consisten en realidad en dos especies distintas (Garrison 1992). Poblaciones de Drosophila lummei Hackman 1972 provenienentes de Rusia, Japón y Suecia pudieron ser claramente diferenciadas en base al análisis morfométrico de sus alas (Haas y Tolley 1998). En triatominos, el DA se ha empleado recientemente en el análisis de las relaciones filogenéticas entre las especies del género Rhodnius sp. (Dujardin et al. 1999). 


\section{Objetivos}

El objetivo de este trabajo es evaluar la utilidad del patrón de hidrocarburos cuticulares en el estudio de la taxonomía de triatominos. Como objetivos específicos se pretende:

1- Determinar la composición y estructura de los hidrocarburos cuticulares en las distintas especies que conforman el subcomplejo Triatoma sordida (T. sordida, T. guasayana, T. garciabesi y T. patagonica), analizando las relaciones inter e intraespecíficas en el mismo.

2- Estudiar la composición de los hidrocarburos cuticulares de Triatoma dimidiata, uno de los tres vectores más importantes de la Enfermedad de Chagas en Latinoamérica, analizando su variabilidad a lo largo de la mayor parte de su distribución geográfica.

En el marco de diversos proyectos internacionales como la ECLAT (Red Europeo Latinoamericana de Investigación en Triatominos), CDIA (Chagas Disease Intervention Activities), y SSA-ATU (American Trypanosomiasis Update) entre otros, se ha estudiado la genética poblacional de triatominos, así como varios aspectos biológicos, con el objetivo de contribuir a los programas de control de la enfermedad y a su mejoramiento. Dentro de este campo, la aplicación del patrón de hidrocarburos cuticulares como marcador taxonómico es novedosa y pretende complementar y/o suplementar la información obtenida a partir de otros marcadores entomológicos como son los análisis citogenéticos, isoenzimáticos, morfométricos y moleculares. 


\section{Materiales y Métodos}

\section{1. Insectos}

Se utilizaron las alas de ejemplares macho y hembra de las distintas especies que integran el subcomplejo $T$. sordida, colectados a través de la colaboración de miembros del proyecto CDIA y por miembros del Servicio Nacional de Chagas Córdoba, en distintas localidades de Argentina, Bolivia, Paraguay y Brasil, como se detalla en la tabla 3 y figura 14. Para los análisis sobre $T$. dimidiata las alas fueron provistas por miembros de la red ECLAT, quienes colectaron los especímenes en las localidades detalladas en la tabla 4 y figura 15. Las ventajas de trabajar con alas radican en su facilidad de transporte y disposición y en que contienen prácticamente solo lípidos cuticulares, por lo que se evita la contaminación con lípidos internos durante el proceso de extracción. Además el empleo de distintas partes del insecto para distintas técnicas (alas para análisis de hidrocarburos y morfométricos, gónadas para los análisis citogenéticos, antenas para el patrón de sensilas antenales, patas para los análsis moleculares, etc.), permite el análisis simultáneo de los mismos ejemplares y una comparación más precisa de las ventajas de cada técnica y la información extra que aporta respecto de las otras.

Tabla 3. Sitios de colecta de los ejemplares del subcomplejo T. sordida estudiados.

\begin{tabular}{|c|c|c|c|c|c|}
\hline Especie & Pais & Localidad & Departamento/Provincia & $\mathrm{n}$ & Año colecta \\
\hline \multirow[t]{18}{*}{ T. sordida } & Argentina & Pirané & Formosa & 13 & 2005 \\
\hline & Bolivia & Apolo & $\mathrm{La} \mathrm{Paz}$ & 7 & 2004 \\
\hline & & Izozog & Santa Cruz & $15^{\mathrm{a}}$ & \\
\hline & & Quillacollo & Cochabamba & 4 & \\
\hline & Brasil & Posse & Goias & 4 & 2004 \\
\hline & & Rio Verde & Mato Grosso & 16 & \\
\hline & & Rondonopolis & Mato Grosso & 8 & \\
\hline & & Sâo Valério do Tocantins & Tocantins & 11 & \\
\hline & Paraguay & Galilea & Boqueron & 10 & $2003 / 2004$ \\
\hline & & Itacurubi & Concepcion & 2 & \\
\hline & & Saladillo & Concepcion & 3 & \\
\hline & & Zorrilla Cue & Gaira & 3 & \\
\hline & & Pirayu & Paraguari & 1 & \\
\hline & & Sapucai & Paraguari & 4 & \\
\hline & & Bethania & Presidente Hayes & 5 & \\
\hline & & Jope & Presidente Hayes & 6 & \\
\hline & & Villa del Rosario & San Pedro & 5 & \\
\hline & & Itacurubi del Rosario & San Pedro & 5 & \\
\hline \multirow[t]{2}{*}{ T. garciabesi } & Argentina & Belgrano & La Rioja & 16 & 2004 \\
\hline & & Avellaneda & Santiago del Estero & 19 & \\
\hline
\end{tabular}




\begin{tabular}{|c|c|c|c|c|c|}
\hline Especie & Pais & Localidad & Departamento/Provincia & $\mathrm{n}$ & Año colecta \\
\hline \multirow[t]{4}{*}{ T. guasayana } & Argentina & Avellaneda & Santiago del Estero & 32 & 2004 \\
\hline & Bolivia & Mataral & Cochabamba & 12 & 2006 \\
\hline & Paraguay & Tiberia & Boqueron & 5 & $2003 / 2004$ \\
\hline & & Jope & Presidente Hayes & 6 & \\
\hline $\operatorname{Tg} x \operatorname{Ts}^{b}$ & Brasil & \multicolumn{2}{|c|}{ Insectario Fund. Instituto Oswaldo Cruz } & 6 & - \\
\hline T. patagonica & Argentina & 9 de Julio & Santa Fe & 21 & 2005 \\
\hline
\end{tabular}

${ }^{\text {a }}$. Izozog grupo 1= 4 insectos, Izozog grupo 2=11; estos insectos coexisten en simpatría en la región de Izozog, pero se separan en grupos en base a análisis isoenzimáticos (Noireau et al. 1998) y a su distinto perfil de hidrocarburos.

b. insectos cruza entre T. guasayana y T. sordida

Figura 14. Mapa detallando los lugares de colección de los ejemplares del subcomplejo $T$. sordida.

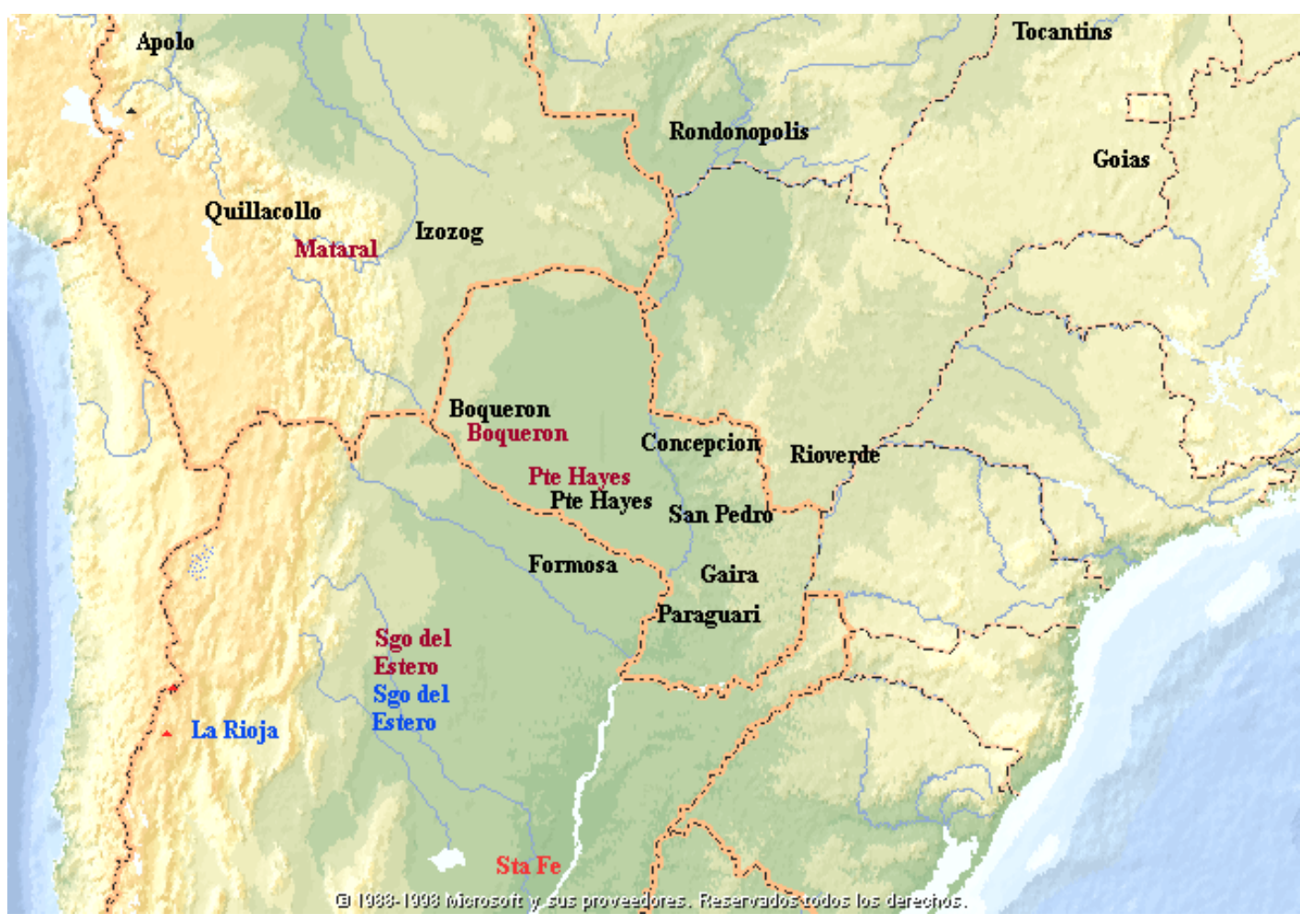

En negro de $T$. sordida, azul de $T$. garciabesi, marrón de $T$. guasayana y rojo de $T$. patagonica. 
Tabla 4. Sitios de colecta de los especímenes de $T$. dimidiata.

\begin{tabular}{|c|c|c|c|c|}
\hline Pais & Localidad & Departamento & $\mathrm{n}$ & Año colecta \\
\hline Belice & Rio Frio & Augustine & 10 & 1971 \\
\hline \multirow[t]{2}{*}{ Colombia } & Boavita & Boyaca & 10 & 2004 \\
\hline & Pore & Casanare & 10 & \\
\hline \multirow[t]{4}{*}{ Costa Rica } & Tres Ríos & Cartago & 3 & 2005 \\
\hline & La Cruz & Guanacaste & 4 & \\
\hline & San Rafael & Heredia & 3 & \\
\hline & Vuelta de Jorco & San Jose & 4 & \\
\hline Honduras & Tegucigalpa & Tegucigalpa & 8 & 2004 \\
\hline \multirow[t]{5}{*}{ Guatemala } & Lanquín & Alta Verapaz & 4 & $2001 / 2002 /$ \\
\hline & Calderas & Jutiapa & 10 & $/ 2004$ \\
\hline & Carpinteros & & & \\
\hline & La Brea & & & \\
\hline & Flores & Petén & 15 & \\
\hline Honduras & Tegucigalpa & Tegucigalpa & 8 & 2004 \\
\hline \multirow[t]{23}{*}{ Mexico } & Belisario Domínguez & Chiapas & 6 & 2004 \\
\hline & Cozumel & Quintana Roo & 6 & $2001 / 2002$ \\
\hline & Acomul & Hidalgo & 16 & $2001 / 2002$ \\
\hline & Pachiquitla & & & \\
\hline & Hueyatetl & & & \\
\hline & Canali & & & \\
\hline & Atalco & & & \\
\hline & La Victoria & Oaxaca & $9^{\mathrm{a}}$ & 2004 \\
\hline & Rió Guasamando & & & \\
\hline & San José Chinantequilla & & & \\
\hline & El Zacatal & & & \\
\hline & Cienaga Grande & & & \\
\hline & S Isidro Campechero & & & \\
\hline & Cinco Negritos & & & \\
\hline & La Luz & Veracruz & 11 & $2001 / 2002$ \\
\hline & Ursulo Galván & & & \\
\hline & Mesa de Tlanchinol & & & \\
\hline & Los Cerritos & & & \\
\hline & Tancolol & & & \\
\hline & El Cantarito & & & \\
\hline & Ekmul & Yucatán & 21 & $2001 / 2002$ \\
\hline & Rancho Loreto & & & \\
\hline & Paraiso & & & \\
\hline
\end{tabular}

${ }^{\mathrm{a}}$ : Oaxaca grupo $1=4$ insectos, Oaxaca grupo 2=5 insectos; se separaron en base a su distinto perfil de hidrocarburos 
Figura 15. Mapa detallando los lugares de colección de los ejemplares de T. dimidiata.

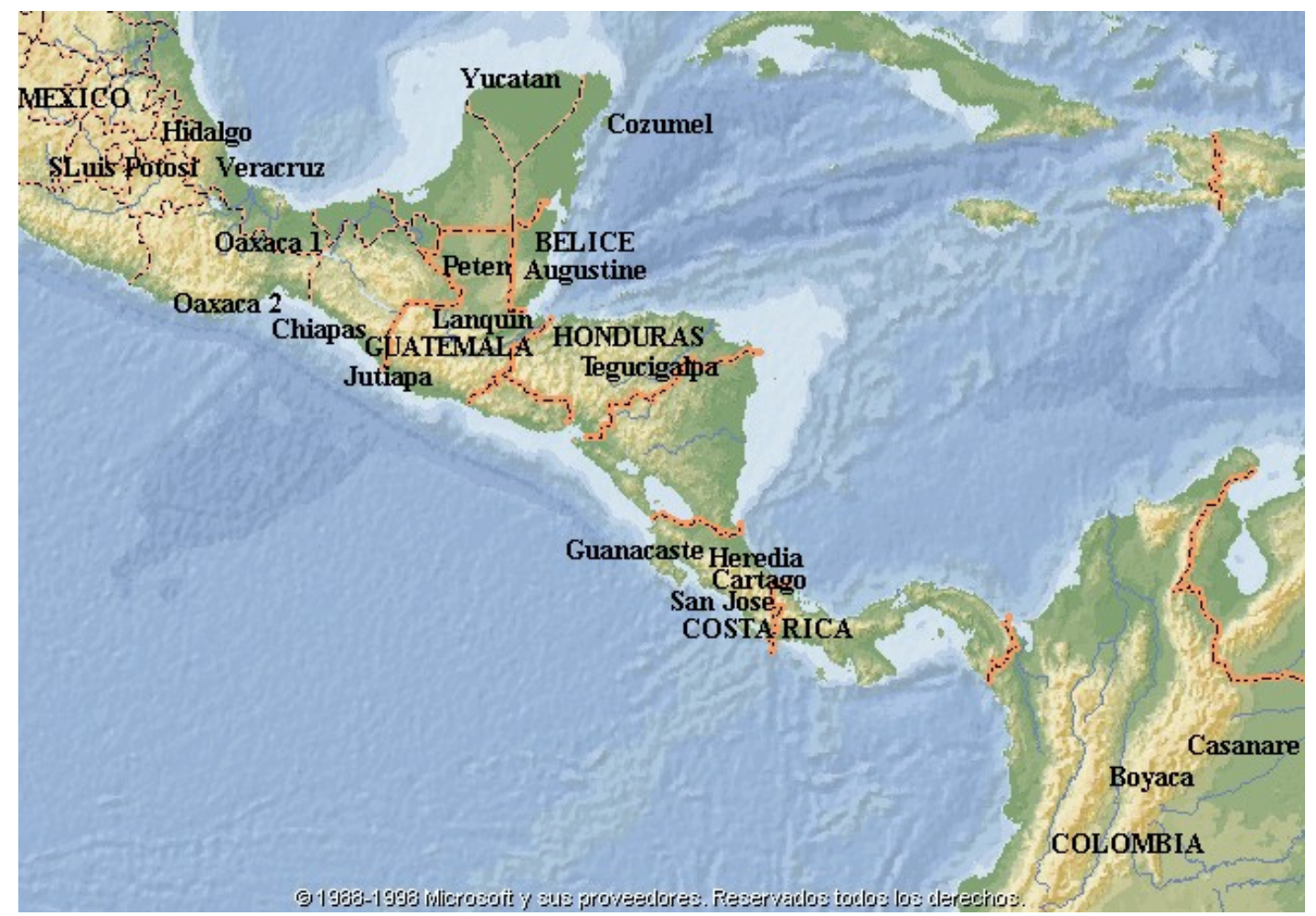

\section{2. Obtención de lípidos cuticulares y purificación de la fracción de hidrocarburos}

Las alas de los insectos fueron lavadas con agua bidestilada para la remoción de contaminantes hidrosolubles y posteriormente los lípidos cuticulares fueron extraídos de las alas mediante inmersión de las mismas en hexano destilado $(6 \mathrm{ml} / \mathrm{g})$ durante $24 \mathrm{~h}$. La fracción de hidrocarburos cuticulares fue separada del resto de los componentes lipídicos mediante cromatografía de adsorción en mini columna con ácido silícico (10 mm x 5 mm I.D.,1malla de $100-200 \mu \mathrm{m})$ previamente activado a $150^{\circ} \mathrm{C}$ durante 1 hora, eluídas con hexano destilado (4 $\mathrm{ml}$ totales).

\section{3. Análisis de los hidrocarburos cuticulares mediante CGC y CGC-MS}

La composición de hidrocarburos fue analizada mediante cromatografía gaseosa capilar (CGC). La cromatografía gaseosa es un método de separación en el cual los componentes de una mezcla se reparten entre dos fases: la fase estacionaria (líquida), que posee una gran superficie de exposición y la fase móvil, un gas inerte que circula en contacto con la fase estacionaria. La muestra se vaporiza en el sistema de inyección y es transportada por la fase móvil gaseosa (gas carrier) a través de la columna. El reparto o partición de los componentes de la mezcla entre las fases se basa en sus diferentes solubilidades en la fase estacionaria a una temperatura dada. Por lo tanto, los componentes de la mezcla (solutos o 
analitos) se separan entre sí en base a sus presiones de vapor relativas y de acuerdo a sus afinidades con la fase estacionaria. Este tipo de proceso cromatográfico se denomina partición. Las partes esenciales de un equipo de CGC se muestran en la figura 16.

Figura 16. Equipo de cromatografía gaseosa

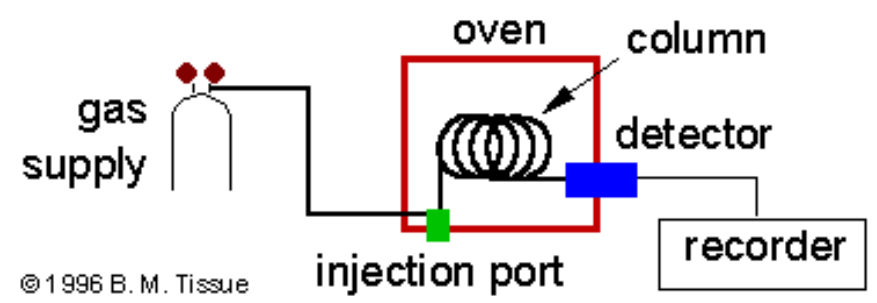

gas supply: fuente de gases, oven: horno, injection port: inyector, column: columna, detector: detector, recorder: registrador

El gas portador (carrier) actúa como fase móvil y su función principal es transportar la muestra a través de la columna. Debe ser inerte en las condiciones usadas y no debe interaccionar químicamente con la muestra, los gases utilizados son hidrógeno, helio y argón.

La muestra a ser analizada, ingresa al sistema cromatográfico a través del puerto inyector. Aquí la muestra es vaporizada mediante la aplicación de una temperatura apropiada y dosificada para el ingreso en la columna cromatográfica, la temperatura del inyector debe ser suficientemente alta como para vaporizar la muestra en forma rápida, pero lo suficientemente baja como para evitar su descomposición térmica o rearreglos químicos. El puerto inyector contiene un septum que permite la inyección con jeringa y a la vez aisla el sistema del ambiente externo, un tubo de vidrio inerte o liner donde la muestra es vaporizada, un sistema de calentamiento para lograr la temperatura de vaporización, un canal de entrada para el gas carrier y una salida hacia la columna. En el caso de grandes cantidades de muestra es común ventear parte de ella para evitar la saturación de la columna (modo split), mientras que en caso de pequeñas cantidades o análisis de trazas (como en las alas de insectos) se suele introducir el total de la muestra inyectada (modo splitless); el inyector para ello tiene una salida accesoria hacia el exterior (figura 17). 
Figura 17. Esquema de un puerto inyector splitless.

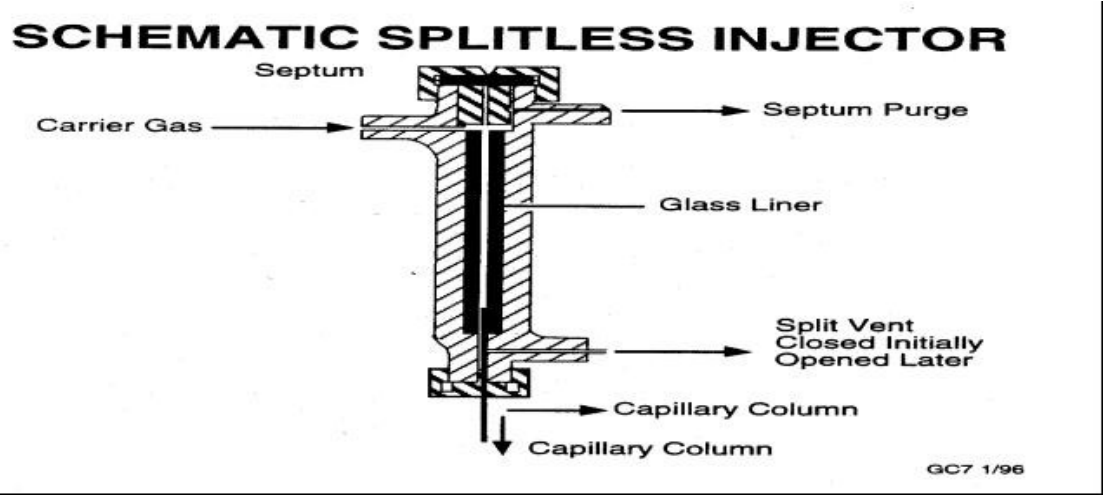

septum: septo, gas carrier: gas portador, septum purge: purga del septum, glass liner: liner de vidrio, split vent: venteo de split (cerrado al inicio, abierto después), capillary column: columna capilar.

Actualmente se utilizan columnas capilares de gran longitud donde la fase estacionaria constituye una película que recubre la superficie interna de la columna. Se tiene que tener una idea aproximada de la identidad de los compuestos a separar ya que la fase estacionaria tiene que tener una polaridad similar a la de los compuestos en estudio. Los distintos componentes de la mezcla que constituye la muestra tendrán distintos grados de interacción con la fase estacionaria, quedando retenidos diferencialmente en ella. La columna se encuentra termostatizada dentro del horno del cromatógrafo de modo que al incrementar la temperatura, en función de su afinidad con la columna, los componentes serán diferencialmente arrastrados por el gas carrier hacia el detector. El detector debe ser sensible a los efluentes de la columna y capaz de suministrar un registro de la cromatografía en la forma de un cromatograma. La señal del detector debe ser proporcional a la cantidad de cada soluto (analito), con lo cuál es posible realizar un análisis cuantitativo. Se han desarrollado una gran cantidad de detectores para monitorear los componentes que son separados en la columna. Los detectores más usados son los de ionización de llama (FID); los analitos que llegan al detector son ionizados por una llama y convertidos en una señal eléctrica que luego de ser amplificada, generará el cromatograma. La temperatura del detector debe ser suficientemente altas como para evitar la condensación de la muestra. Si la temperatura es muy baja y ocurre condensación, se producirá ensanchamiento de los picos o la ausencia total de los mismos. El FID es el detector mas utilizado, ya que cumple con todos los requerimientos de un buen detector para cromatografía gaseosa: alta sensibilidad, muy buena estabilidad, respuesta rápida (1 msec), bajo volumen muerto $(1 \mathrm{~mL})$ y amplia respuesta linear. 
Figura 18. Esquema de un detector de ionización de llama (FID)
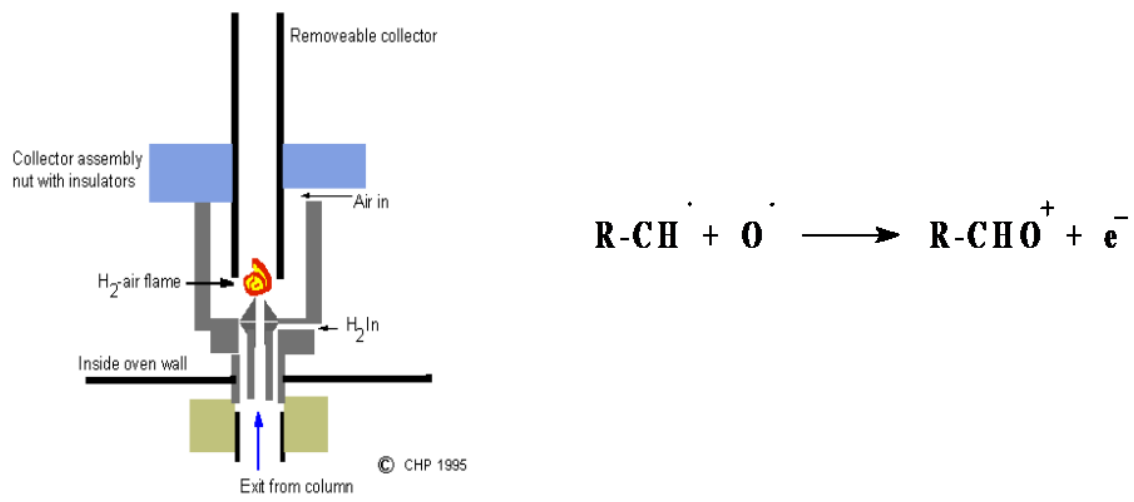

Para el presente análisis se utilizó un cromatógrafo Hewlett-Packard 6890 con FID operado a $320^{\circ} \mathrm{C}$; el inyector operado en modo splitless a $310^{\circ} \mathrm{C}$; una columna no polar $\mathrm{HP}-5$ (30 m x $0.32 \mathrm{~mm}$ I.D., Hewlett-Packard). La temperatura del horno fue programada a $50^{\circ} \mathrm{C}$ durante $2^{\prime}$, elevada hasta $180^{\circ} \mathrm{C}$ a $20^{\circ} \mathrm{C} / \mathrm{min}$., y luego hasta $310^{\circ} \mathrm{C}$ a $3^{\circ} \mathrm{C} / \mathrm{min}$. (tiempo final $\left.10^{\prime}\right)$.

Para el cálculo del KI, se inyectáron estándares de hidrocarburos normales de longitud de cadena conocida en las condiciones cromatográficas de resolución de la muestra, y luego se graficó el tiempo de elución versus la longitud de cadena. De esta manera por interpolación del tiempo de elución un hidrocarburo de número de carbonos desconocido en la ecuación obtenida a partir del gráfico, se obtuvo su valor de KI. Mediante la inyección de estándares de hidrocarburos de 22 a 44 átomos de carbono corridos en las mismas condiciones cromatográficas de las muestras, se calcularon los KI de los distintos componentes, aplicando la siguiente fórmula:

$\mathrm{KI}=100 *(\mathrm{Ca}+(\mathrm{Cb}-\mathrm{Ca}) *((\operatorname{trX}-\operatorname{tra}) /(\operatorname{trb}-\operatorname{tra})))$

donde: Ca: número de carbonos del estándar que eluye anteriormente

Cb: número de carbonos del estándar que eluye posteriormente

trX: tiempo de retención del hidrocarburo incógnita

tra: tiempo de retención del estándar que eluye anteriormente

trb: tiempo de retención del estándar que eluye posteriormente.

La abundancia relativa de cada hidrocarburo en cada espécimen se obtuvo dividiendo el área bajo cada pico en el cromatograma sobre el área total, estos datos proveen información sobre las variaciones cuantitativas entre los grupos analizados y constituyen los datos de entrada para los análisis taxonómicos posteriores. 
La determinación de la estructura de los hidrocarburos se efectuó por cromatografía gaseosa capilar acoplada a espectrometría de masas (CGC-MS) en cromatógrafo HewlettPackard 6890 acoplado a un espectrómetro de masas quadrupolo Agilent 5975C, operado a $70 \mathrm{eV}$, con la línea de transferencia a $330^{\circ} \mathrm{C}$, la fuente de iones a $230^{\circ} \mathrm{C}$ y el quadrupolo a $150^{\circ} \mathrm{C}$; inyector en modo splitless a $310^{\circ} \mathrm{C}$; columna no polar HP-5MS (30 m x $0.25 \mathrm{~mm}$ I.D., Agilent); temperatura del horno programada a $50^{\circ} \mathrm{C}$ durante $2^{\prime}$, llevada hasta $180^{\circ} \mathrm{C}$ a $20^{\circ} \mathrm{C} / \mathrm{min}$., luego hasta $310^{\circ} \mathrm{C}$ a $3^{\circ} \mathrm{C} / \mathrm{min}$. (tiempo final $10^{\prime}$ ). La asignación de las mismas se efectuó según se describe en Juárez et al. 2001.

\section{4. Análisis taxonómico}

A fin de evaluar la variabilidad entre los insectos y determinar las similitud entre individuos y entre grupos, se analizaron las muestras mediante análisis discriminante canónico lineal. Este se usa principalmente para clasificar individuos o unidades experimentales en dos o más poblaciones definidas de manera única. Se crean nuevas variables, las funciones discriminantes, que contienen toda la información útil para la discriminación, disponible en las variables originales. El análisis discriminante es similar al de regresión, excepto que la variable dependiente es categórica en lugar de continua. Asimismo es similar al análisis de la varianza de una vía, en el sentido que se determina si dos o más grupos son significativamente diferentes entre sí, en los valores de sus medias respecto a una variable particular. En este caso, la significancia de dicha variable en la discriminación entre los grupos está dada por el test F, la razón de la varianza entre grupos y la varianza total dentro de los grupos. Si la varianza entre grupos es significativamente alta, hay diferencias significativas entre sus medias y se puede establecer que la variable es útil para discriminar entre los mismos. Usualmente múltiples variables son incluídas en un estudio, a fin de evaluar cuáles contribuyen a la diferenciación entre grupos. En este caso se tendrán matrices de varianzas-covarianzas entre y dentro de los grupos las cuales se compararán mediante un test F multivariado.

Cuando varias variables son consideradas a fines de discriminación, cabe analizar si es necesario incluir todas las variables en el análisis, o si unas variables son mejores que otras para discriminar. Para el análisis de los grupos de triatominos en estudio, se probaron los métodos de eliminación hacia adelante y hacia atrás. En el método de eliminación hacia adelante, la variable que produce el valor más grande de $\mathrm{F}$ en un ANOVA de una vía es seleccionada primero, siempre y cuando su valor sea significativo en un nivel seleccionado por el investigador. La siguiente variable seleccionada es aquella que produce el valor más grande de $\mathrm{F}$ en un análisis de covarianza (ANCOVA) de una sola vía, utilizando la primer 
variable seleccionada como covariable. Las subsiguientes variables se seleccionan de la misma manera, incluyendo las previamente elegidas como covariables, hasta que ya ninguna alcance el nivel de significancia elegido por el investigador; deteniéndose ahí el método. En el método de eliminación hacia atrás, se empieza con la inclusión de todas las variables; luego se elimina la variable menos útil tras identificarla mediante un ANCOVA para cada variable, utilizando las otras como covariables. Así se van eliminando todas las variables hasta que queden solo las estadísticamente significativas al nivel de $\mathrm{F}$ elegido por el investigador.

En los análisis efectuados, las matrices de estructura son mostradas como medio de visualizar la contribución de las variables a cada función discriminante. Los coeficientes de la matriz de estructura son los valores de correlación entre las variables originales y las funciones discriminantes, por lo que a mayor valor de correlación, mayor contribución de la variable a la función discriminante.

Como medida de significancia de la diferencia entre los grupos se eligió emplear el $\lambda$ de Wilks y su valor de probabilidad (p) asociado (Wilks 1932). Este estadístico, resulta de la razón entre la suma de los cuadrados dentro de los grupos y la suma total de los cuadrados y se calcula como sigue:

Wilk's Lambda $=\operatorname{det}(\mathrm{W}) / \operatorname{det}(\mathrm{T})$

donde: det: determinante

$\mathrm{W}$ : matriz de varianza/covarianza dentro de los grupos

T: matriz de varianza/covarianza total.

Su valor oscila entre 1 (la media de todos los grupos es igual, es decir no hay discriminación) y 0 (hay discriminación perfecta entre los grupos).

Como forma de evaluar la exactitud de las funciones de clasificación, se empleó el método de validación cruzada (Lachenbruch y Mickey 1968). En este método, en sucesivos pasos se quita cada dato del análisis, se crean funciones discriminantes y de clasificación con los datos restantes, las cuáles luego se utilizan para clasificar el dato quitado. Un valor similar en los porcentajes de clasificación original y de validación cruzada, indica que las funciones discriminantes son idóneas para clasificar los datos.

Las relaciones entre los grupos y la estructura poblacional de las especies resultantes del DA, se visualizan mediante dendrogramas usando como técnica de agrupamiento, el agrupamiento de a pares no ponderado basado en la media aritmética de los coeficientes de similitud de los pares en unión (UPGMA). Como coeficientes de similitud para aplicar el método UPGMA se utilizó la matriz de distancias de Mahalanobis entre los centroides de los grupos, derivada del análisis discriminante. Esta distancia permite cuantificar la similitud 
entre elementos tanto en un espacio formado por variables independientes (ortogonales), como con variables correlacionadas. Su ecuación es la siguiente:

$\mathrm{M}^{2}=(\mathrm{n}-1)^{*}(\mathrm{Xd}-\mathrm{Xm})^{*} \mathrm{C}-1^{*}(\mathrm{Xd}-\mathrm{Xm})^{\prime}$

donde: $n$ : es el número de casos

$\mathrm{Xd}$ : es la matriz de datos originales para las variables independientes

$\mathrm{Xm}$ : es la matriz de medias para las variables independientes

$\mathrm{C}-1$ : es la inversa de la matriz de varianza/covarianza para las variables independientes

Previo al DA, se empleó el análisis de componentes principales (PCA) como forma preliminar de visualizar las relaciones entre los especímenes individuales y como medio de detectar ejemplares "outliers", que puedan afectar el DA. Estas relaciones se muestran en figuras donde los ejemplares se grafican tanto sobre las tres primeras componentes principales, como mediante dendrogramas usando como técnica de agrupamiento el UPGMA; como coeficiente de similitud para aplicar el método UPGMA se utilizó la distancia promedio entre los centroides de los grupos, según la siguiente fórmula:

$\mathrm{DP}=\sqrt{ }\left(\left(\sum \text { distancias entre todos los centroides }\right)^{2} /\right.$ cantidad de grupos $)$

Para eliminar la interdependencia de los valores de porcentaje de área de los hidrocarburos utilizados como variables, relación que afecta los análisis multivariados empleados, se aplicó sobre los mismos la transformación arco seno (Sokal y Rohlf 2001) para cada ejemplar, según la siguiente expresión:

$\%$ área transformado $=\operatorname{arcoseno}(\sqrt{ }(\%$ área $/ 100))$. 


\section{Resultados}

\section{1. Subcomplejo Triatoma sordida.}

IV. 1. 1. Identificación estructural de los hidrocarburos cuticulares mediante CGC-MS

Se analizó mediante CGC-MS el patrón de hidrocarburos cuticulares de las cuatro especies que integran el subcomplejo. En la figura 19 se muestra un cromatograma de $T$. garciabesi con la identificación de los picos cromatográficos que contienen los hidrocarburos determinados mediante CGC-MS, mientras que en la tabla 5 se detalla la lista de hidrocarburos identificados con su respectivo KI e iones diagnósticos. Se utiliza como ejemplo un cromatograma de esta especie, ya que es la que presenta la mayor cantidad de componentes; como se detalla en el punto IV.1.2, no todas las especies presentan los mismos hidrocarburos.

Figura 19. Perfil de CGC de T. garciabesi con los picos cromatográficos correspondientes a los hidrocarburos identificados por CGC-MS en la tabla 5.

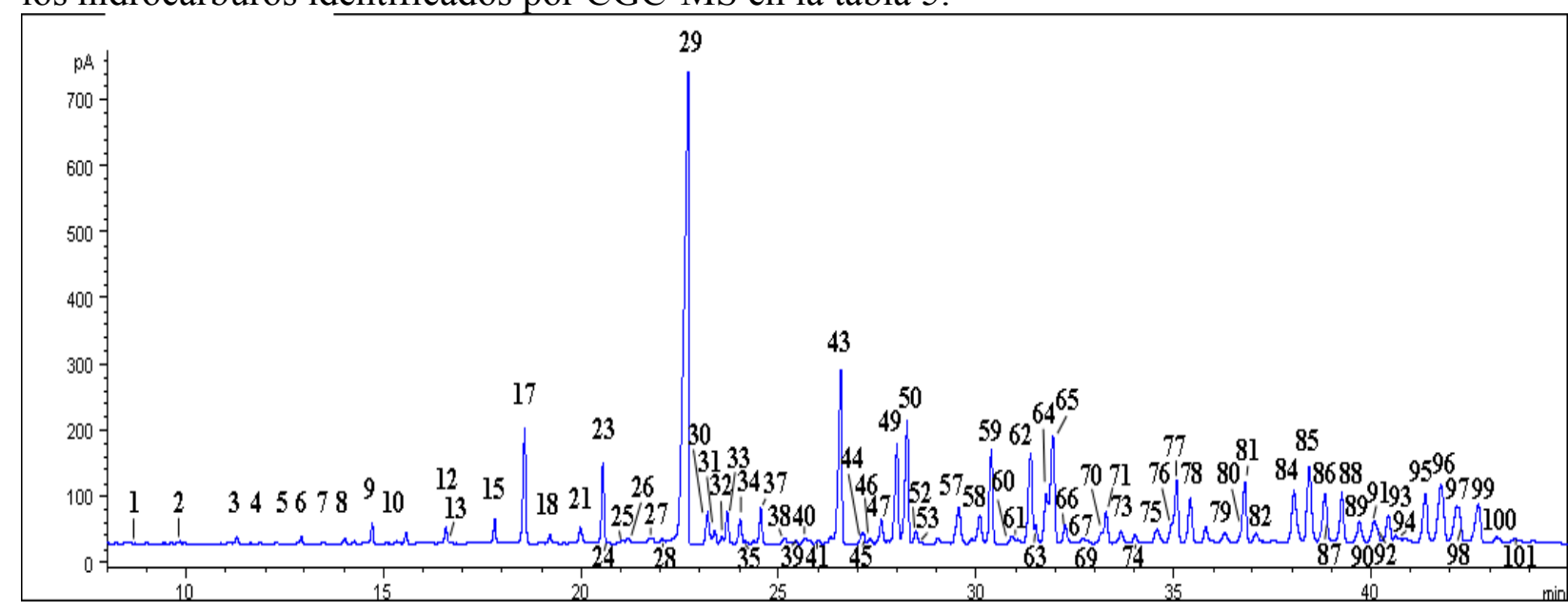

Tabla 5. Hidrocarburos cuticulares del subcomplejo T. sordida identificados mediantes CGCMS.

[ LA TABLA INICIA EN LA SIGUIENTE HOJA ]. 
$1 n-\mathrm{C} 21$

$2 n-\mathrm{C} 22$

$3 n-\mathrm{C} 23$

4 11- + 9- metil C23

5 3,9- + 3,13- dimetil C23

$6 n-\mathrm{C} 24$

7 11- + 12- metil C24

8 4- metil C24

$9 n-\mathrm{C} 25$

10 11- + 13- + 9-metil C25

11 3-metil C25

$12 n-\mathrm{C} 26$

13 3,13-dimetil C25

14 12- + 11- + 13- + 10-metil C26

15 4- metil C26

16 3-metil C26

$17 n-\mathrm{C} 27$

18 11- + 13- + 7- + 9- metil C27

19 5- metil C27

20 11,15-dimetil C27

21 3- metil C27

22 5,13- + 5,15- dimetil C27

$23 n-\mathrm{C} 28$

24 3,17- + 3,15- + 3,7- dimetil C27

25 11- + 12- + 13-+ 14- metil C28

26 6- metil C28

27 4- metil C28

28 3-metil C28

$29 n-\mathrm{C} 29$

30 13- + 11- + 9- + 15- metil C29
2100

2200

2300

$168 / 169$ 196/197140/141323338

$2392 \quad 154 / 155,224 / 225 ; 168 / 169,196 / 197,323$

$2400 \quad 338$

$2431 \quad 168 / 169210 / 211,182 / 183196 / 197,337$

$2460 \quad 71308 / 309337352$

$2500 \quad 352$

$2535 \quad 168 / 169196 / 197224 / 225140 / 141 \quad 252 / 253351366$

$2574 \quad 337365$

$2600 \quad 366$

$2605 \quad 57196 / 197209351$

$2632182 / 183224 / 225,168 / 169238 / 239,196 / 197210 / 211$, $154 / 155252 / 253380$

$2660 \quad 336 / 337380$

$2672 \quad 351380$

$2700 \quad 380$

$2732 \quad 168 / 169252 / 253,196 / 197224 / 225,140 / 141280 / 281$

$112 / 113308 / 309379394$

$2750 \quad 84 / 85336 / 337380$

$2760 \quad 168 / 169196 / 197239267393$

$2772 \quad 364 / 365394$

$2780 \quad 85224 / 225211,196 / 197239351407$

$2800 \quad 394$

$2805 \quad 168 / 169267,196 / 197239,308 / 309127,379407$

$2826 \quad 168 / 169266 / 267,182 / 183252 / 253,196 / 197238 / 239$, $210 / 211224 / 225,393$

$2850 \quad 98 / 99336 / 337379$

$2858 \quad 70 / 71364 / 365407$

$2864 \quad 57379407$

$2900 \quad 408$

$2932 \quad 196 / 197252 / 253,168 / 169280 / 281,140 / 141308 / 309$, $224 / 225,422$ 
Continuación tabla 5.

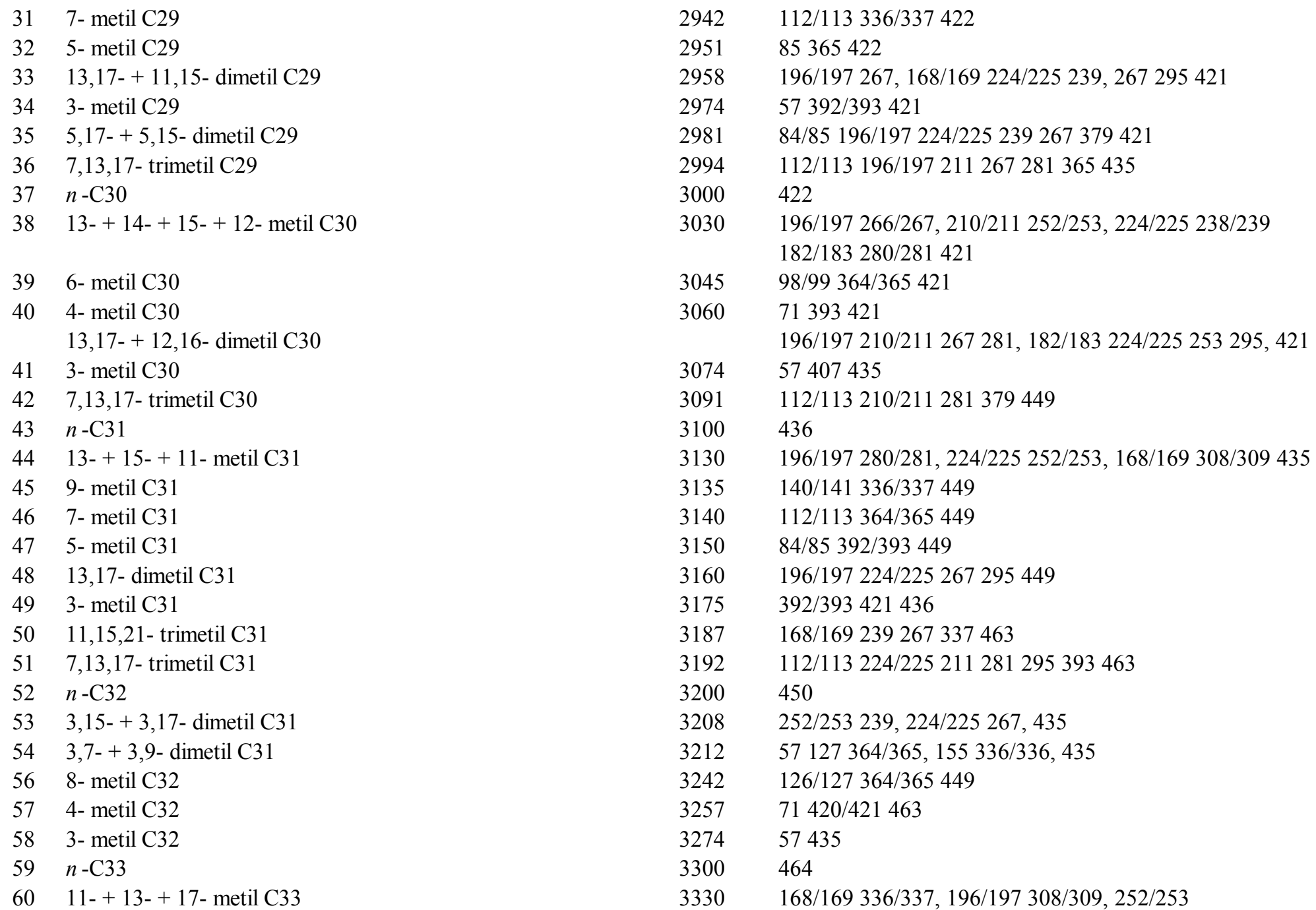


continuación tabla 5.

\begin{tabular}{|c|c|c|c|}
\hline \multirow{3}{*}{$\begin{array}{l}61 \\
62\end{array}$} & 9- metil C33 & 3341 & $140 / 141364 / 365$ \\
\hline & 5- metil C33 & 3351 & $84 / 85421$ \\
\hline & 13,17- + 15,19- dimetil C33 & & 196/197 252/253 $267323,224 / 225295,477$ \\
\hline 63 & 11,21- dimetil C33 & 3357 & 168/169 196/197323351477 \\
\hline 64 & 3- metil C33 & 3372 & $448 / 449420 / 421477$ \\
\hline \multirow{2}{*}{65} & $5,17-+5,19-$ dimetil $\mathrm{C} 33$ & 3378 & $85252 / 253267,224 / 225295,435$ \\
\hline & \multicolumn{2}{|l|}{ 11,17,21- trimetil C33 } & 168/169 196/197267337365 491 \\
\hline 66 & $n-\mathrm{C} 34$ & 3400 & 478 \\
\hline 67 & $3,17-+3,19-+3,11-$ dimetil C33 & 3404 & $252 / 253267,224 / 225295,336 / 337183,463491$ \\
\hline 68 & 3,7- + 3,9- dimetil C33 & 3408 & $127392 / 393,155364 / 365,463477$ \\
\hline 69 & 10- metil C34 & 3425 & $154 / 155364 / 365491$ \\
\hline \multirow[t]{2}{*}{70} & 4- metil C34 & 3460 & 71449491 \\
\hline & \multicolumn{2}{|l|}{ 12,22-dimetil C34 } & 182/183 196/197337351505 \\
\hline 71 & 6,10- + 6,18- dimetil C34 & 3464 & $98 / 99,364 / 365169,252 / 253281,435491$ \\
\hline 72 & $12,16,22-+12,16,20-$ trimetil C34 & 3472 & 182/183 $253295,196 / 197351,224 / 225323,365491$ \\
\hline 73 & 4,18- + 4,20- dimetil C34 & 3480 & $70 / 71,252 / 253281,224 / 225309,463491$ \\
\hline 74 & 6,10,14- trimetil C34 & 3500 & 98/99 308/309 169239379449505 \\
\hline 75 & 11- + 13- metil C35 & 3527 & $168 / 169364 / 365,196 / 197336 / 337,505$ \\
\hline 76 & 11,23-dimetil C35 & 3548 & 168/169 196/197351505 \\
\hline 77 & 7,11- dimetil C35 & 3554 & $112 / 113364 / 365183435$ \\
\hline \multirow[t]{2}{*}{78} & 13,17,23- trimetil C35 & 3575 & 196/197267295365 519 \\
\hline & \multicolumn{2}{|l|}{ 5,17- dimetil C35 } & $85280 / 281267463519$ \\
\hline 79 & $8,16-+8,12$ - dimetil C36 & 3625 & $126 / 127,308 / 309253,364 / 365197,435$ \\
\hline \multirow[t]{2}{*}{80} & $12,16-+12,24-$ dimetil C36 & 3650 & $182 / 183,308 / 309253,196 / 197365,379$ \\
\hline & \multicolumn{2}{|l|}{ 8,12,16- trimetil C36 } & 126/127 308/309 197267379449 \\
\hline 81 & 4-metil C36 & 3654 & 71477505 \\
\hline 82 & 6,10 - dimetil C36 & 3670 & 98/99 392/393169463519 \\
\hline 83 & 4,20- + 4,16- dimetil C36 & 3680 & $70 / 71,252 / 253309,308 / 309253,491$ \\
\hline 84 & $11-+13-+15-+17-$ metil C37 & 3725 & $\begin{array}{l}\text { 168/169 392/393, 196/197 364/365, 224/225 336/337, } \\
252 / 253519\end{array}$ \\
\hline \multirow[t]{2}{*}{85} & \multirow[t]{2}{*}{$13,17-+11,21-+13,23-+15,19-$ dimetil C37 } & 3750 & 196/197 308/309 267 379, 168/169 252/253 323 407, \\
\hline & & & $\begin{array}{l}196 / 197 \quad 224 / 225351379,224 / 225 \text { 280/281 } 295351 \\
112 / 113392 / 393183463\end{array}$ \\
\hline
\end{tabular}


continuación tabla 5.

$8613,17,23-+13,17,21-+15,19,23-$ trimetil C37

$87 \quad 11,21,25-$ trimetil C37

5,13- + 5,15- + 5,17- dimetil C37

88 3,15- + 3,17- dimetil C37

89 12- + 14- + 16- + 18- + 19- metil C38

$903,7,11-+3,7,15-$ trimetil C37

91 14,18- + 16,20- dimetil C38

92 12,22- dimetil C38

$936,10-+6,16-+6,18-$ dimetil C38

$14,18,24-+12,16,22-+14,18,22-$ trimetil C38

94 4,16- + 4,14- + 4,18- dimetil C38

95 13- + 11- + 15- + 17- + 19- metil C39

$9615,19-+13,23-+13,19-+15,21-+15,23-+$ $11,21-+13,25-$ dimetil C39

97 7,11- dimetil C39

$13,19,25-+15,19,23-+13,17,23-$ trimetil C39

98 5,17- + 5,15- + 5,19- + 5,13- dimetil C39

99 3,17- + 3,15- + 3,19- dimetil C39

100 14,18,24- trimetil C40

101 13- + 11- + 15- + 17- + 19- + 21- metil C41
196/197, 224/225 365, 252/253 337, 267323393 , 224/225 295365,547

$168 / 169196 / 197267323393421$

85 364/365 211, 85 336/337 239, 85 308/309 267, 491

$57,336 / 337239,308 / 309267,519$

182/183 392/393, 210/211 364/365, 238/239 336/337,

266/267 308/309, 280/281, 533

57 127, 392/393 197, 336/337 253, 463533

210/211 308/309281379, 238/239 280/281309351 547

$182 / 183252 / 253337407547$

98/99, 420/421 169, 336/337 253, 308/309 281, 491

210/211 224/225 $281323379393,182 / 183$ 252/253

$253351421,210 / 211252 / 253281323351393547$

70/71, 336/337 253, 364/365 225, 308/309 281, 519547

196/197 392/393, 168/169 420/421, 224/225 364/365,

$252 / 253336 / 337,280 / 281308 / 309547$

224/225 308/309 295 379, 196/197 252/253 351407 ,

196/197 308/309 295 407, 224/225 280/281323 379, 224/225 252/253 351 379, 168/169 280/281323 435,

$196 / 197224 / 225379407561$

$112 / 113420 / 421183491$

196/197 224/225 295323393 421, 224/225 252/253 295

323365 393, 196/197 252/253 267351365 421, 561

$84 / 85,336 / 337267,364 / 365239,392 / 393211,308 / 309295,519$

$57,336 / 337267,364 / 365239,308 / 309295,547575$

$210 / 211252 / 253281351379421$

196/197 420/421, 168/169 448/449, 224/225 392/393, $252 / 253364 / 365,280 / 281336 / 337,308 / 309,575$

Picos 36, 42 y 51 presentes solamente en T. garciabesi; pico 98 e isomeros 5,x-dimetil del pico 87 presentes en gran abundancia en $T$. guasayana y T. patagonica y a nivel de trazas en $T$. sordida y $T$. garciabesi. 
La mezcla de hidrocarburos identificada, comprende un centenar de cadenas lineales y metil ramificadas (con una a tres sustituciones). Los $n$-alcanos consistieron en una serie contínua de cadenas de 21 a 34 carbonos, caracterizados por un predominio de las cadenas impares, sobre todo de 25 a 33 carbonos.

La fracción de monometilalcanos, consiste en cadenas principales pares e impares donde las ramificaciones de las cadenas impares se ubican en posición impar, mientras que en las pares se ubica preferencialmente en posición par aunque también impar (ej. 11- + 12metil C24, 12- + 11- + 13- + 10- metil C26, entre otros). Las ramificaciones en posiciones terminales y cercanas a éstas (posiciones 5 a 9) muestran una abundancia similar a los monometilalcanos internos en cadenas principales de 23 a 34 carbonos, sin embargo en cadenas más largas los monometilalcanos terminales presentan una franca disminución (quizas con excepción del 4-metil C36), estándo prácticamente ausentes en los componentes de 38 a 42 carbonos, formados por monometilalcanos internos. En las cadenas pares se destacan de manera característica los isómeros 4-metil en cadenas lineales de 24 a 36 carbonos y los 3-metil en cadenas lineales de 26 a 32 carbonos, con los isomeros 12-metil como los metilalcanos internos más comunes (en cadenas de 24 a 38 carbonos). En las cadenas impares predominan las ramificaciones terminales 3-metil en cadenas de 25 a 33 carbonos, 5-metil con la misma longitud e internas 11-y 13-metil en cadenas lineales desde 23 a 41 carbonos.

En los dimetilalcanos las ramificaciones se ubican comúnmente en posiciones pares o impares dependiendo si la cadena lineal tiene número par o impar de carbonos respectivamente. En cadenas impares son comunes los isómeros 3,X-dimetil en cadenas de 23 a 39 carbonos, y 5,X-dimetil en cadenas de 27 a 39 carbonos, con la segunda ramificación en posición interna (3,15-, 3,17-, 3,7-, 3,9-, 5,17-, 5,19-); los dimetilalcanos impares internos consisten generalmente en cadenas con ramificaciones 13,X-, 11,X- y 15,X-. En cadenas pares son comunes las ramificaciones $12, \mathrm{X}-\mathrm{,}, 6, \mathrm{X}-\mathrm{y} 4, \mathrm{X}$ en cadenas de 30 y de 34 a 38 carbonos.

Los trimetilalcanos muestran una abundancia de estructuras correlacionada con el incremento en la longitud de la cadena lineal encontrándose prácticamente ausentes en cadenas lineales menores a 31 átomos de carbono. Las cadenas impares cuentan de manera común con ramificaciones en posiciones 11,X,Y-, 13, X,Y- y 15,X,Y- en cadenas de 31 a 39 carbonos, mientras que las cadenas pares en posiciones $12, \mathrm{X}, \mathrm{Y}-, 14, \mathrm{X}, \mathrm{Y}-$ en cadenas de $34 \mathrm{a}$ 40 carbonos. De manera particular, en $T$. garciabesi se encuentran el isómero 7,13,17- en alcanos C29, C30 y C31. 
IV. 1. 2. Análisis de los hidrocarburos cuticulares mediante CGC, composición en función de las especies y las localidades de colecta.

En las figuras 20,21, 22, 23 y 24 se muestran los perfiles cromatográficos representativos de las distintas especies analizadas, así como de los representantes de una misma especie colectados en distintas localidades; mientras que en la tabla 6 se computan las cantidades relativas de los hidrocarburos identificados.

Tabla 6a. Composición de los HC cuticulares de T. sordida.

\begin{tabular}{|c|c|c|c|c|c|c|c|c|c|c|}
\hline \multirow[b]{2}{*}{ KI } & \multicolumn{2}{|c|}{ Formosa } & \multicolumn{2}{|c|}{ Apolo } & \multicolumn{2}{|c|}{ Izozog G1 } & \multicolumn{2}{|c|}{ Izozog G2 } & \multicolumn{2}{|c|}{ Quillacollo } \\
\hline & media & es & media & es & media & es & media & es & media & es \\
\hline 2100 & 0.0 & 0.0 & 0.0 & 0.0 & 0.0 & 0.0 & 0.0 & 0.0 & 0.0 & 0.0 \\
\hline 2200 & 0.2 & 0.0 & 0.1 & 0.1 & 0.0 & 0.0 & 0.0 & 0.0 & 0.0 & 0.0 \\
\hline 2300 & 0.2 & 0.0 & 0.2 & 0.1 & 0.0 & 0.0 & 0.1 & 0.0 & 0.0 & 0.0 \\
\hline 2330 & 0.0 & 0.0 & 0.0 & 0.0 & 0.0 & 0.0 & 0.0 & 0.0 & 0.0 & 0.0 \\
\hline 2392 & 0.0 & 0.0 & 0.0 & 0.0 & 0.0 & 0.0 & 0.0 & 0.0 & 0.0 & 0.0 \\
\hline 2400 & 0.2 & 0.0 & 0.3 & 0.1 & 0.1 & 0.0 & 0.9 & 0.8 & 0.1 & 0.0 \\
\hline 2435 & 0.1 & 0.0 & 0.1 & 0.1 & 0.1 & 0.1 & 0.1 & 0.0 & 0.0 & 0.0 \\
\hline 2460 & 0.0 & 0.0 & 0.0 & 0.0 & 0.0 & 0.0 & 0.0 & 0.0 & 0.0 & 0.0 \\
\hline 2500 & 0.7 & 0.1 & 0.6 & 0.2 & 0.2 & 0.0 & 0.6 & 0.1 & 1.5 & 0.4 \\
\hline 2532 & 0.4 & 0.1 & 0.1 & 0.1 & 0.1 & 0.1 & 0.0 & 0.0 & 0.1 & 0.0 \\
\hline 2570 & 0.0 & 0.0 & 0.0 & 0.0 & 0.0 & 0.0 & 0.0 & 0.0 & 0.0 & 0.0 \\
\hline 2600 & 0.8 & 0.1 & 0.4 & 0.1 & 0.2 & 0.0 & 0.6 & 0.1 & 0.5 & 0.1 \\
\hline 2605 & 0.0 & 0.0 & 0.0 & 0.0 & 0.0 & 0.0 & 0.0 & 0.0 & 0.0 & 0.0 \\
\hline 2632 & 0.0 & 0.0 & 0.0 & 0.0 & 0.0 & 0.0 & 0.0 & 0.0 & 0.0 & 0.0 \\
\hline 2662 & 0.7 & 0.1 & 0.0 & 0.0 & 0.0 & 0.0 & 0.4 & 0.1 & 0.0 & 0.0 \\
\hline 2672 & 0.0 & 0.0 & 0.0 & 0.0 & 0.0 & 0.0 & 0.0 & 0.0 & 0.0 & 0.0 \\
\hline 2700 & 9.1 & 0.8 & 5.3 & 0.7 & 7.3 & 1.2 & 6.0 & 0.7 & 4.8 & 0.4 \\
\hline 2732 & 0.1 & 0.0 & 0.1 & 0.1 & 0.5 & 0.1 & 0.0 & 0.0 & 0.0 & 0.0 \\
\hline 2750 & 0.0 & 0.0 & 0.0 & 0.0 & 0.0 & 0.0 & 0.0 & 0.0 & 0.0 & 0.0 \\
\hline 2760 & 0.0 & 0.0 & 0.0 & 0.0 & 0.0 & 0.0 & 0.0 & 0.0 & 0.0 & 0.0 \\
\hline 2772 & 1.2 & 0.2 & 0.2 & 0.1 & 0.4 & 0.1 & 0.3 & 0.1 & 1.5 & 0.2 \\
\hline 2780 & 0.1 & 0.0 & 0.0 & 0.0 & 0.0 & 0.0 & 0.0 & 0.0 & 0.0 & 0.0 \\
\hline 2800 & 3.5 & 0.2 & 3.8 & 0.2 & 5.6 & 0.3 & 5.9 & 1.5 & 2.4 & 0.4 \\
\hline 2805 & 0.0 & 0.0 & 0.0 & 0.0 & 0.0 & 0.0 & 0.0 & 0.0 & 0.0 & 0.0 \\
\hline 2831 & 0.0 & 0.0 & 0.1 & 0.1 & 0.0 & 0.0 & 0.0 & 0.0 & 0.1 & 0.1 \\
\hline 2844 & 0.0 & 0.0 & 0.0 & 0.0 & 0.0 & 0.0 & 0.0 & 0.0 & 0.0 & 0.0 \\
\hline 2857 & 0.4 & 0.0 & 0.4 & 0.1 & 2.4 & 0.3 & 0.1 & 0.0 & 1.1 & 0.2 \\
\hline 2861 & 0.1 & 0.0 & 0.0 & 0.0 & 0.1 & 0.1 & 0.1 & 0.0 & 0.0 & 0.0 \\
\hline
\end{tabular}




\begin{tabular}{|c|c|c|c|c|c|c|c|c|c|c|}
\hline \multirow[b]{2}{*}{$\mathrm{KI}$} & \multicolumn{2}{|c|}{ Formosa } & \multicolumn{2}{|c|}{ Apolo } & \multicolumn{2}{|c|}{ Izozog G1 } & \multicolumn{2}{|c|}{ Izozog G2 } & \multicolumn{2}{|c|}{ Quillacollo } \\
\hline & media & es & media & es & media & es & media & es & media & es \\
\hline 2905 & 25.6 & 1.9 & 30.4 & 1.5 & 37.3 & 3.3 & 27.4 & 1.5 & 16.8 & 1.8 \\
\hline 2932 & 0.2 & 0.0 & 0.4 & 0.1 & 0.3 & 0.1 & 0.0 & 0.0 & 0.1 & 0.0 \\
\hline 2941 & 0.0 & 0.0 & 0.0 & 0.0 & 0.0 & 0.0 & 0.0 & 0.0 & 0.1 & 0.0 \\
\hline 2951 & 0.1 & 0.0 & 0.3 & 0.0 & 0.3 & 0.0 & 0.0 & 0.0 & 0.4 & 0.0 \\
\hline 2958 & 0.5 & 0.1 & 0.1 & 0.1 & 0.7 & 0.1 & 0.0 & 0.0 & 0.0 & 0.0 \\
\hline 2974 & 5.0 & 0.6 & 1.2 & 0.2 & 0.8 & 0.1 & 1.3 & 0.1 & 2.8 & 0.3 \\
\hline 2982 & 0.0 & 0.0 & 0.0 & 0.0 & 0.0 & 0.0 & 0.0 & 0.0 & 0.0 & 0.0 \\
\hline 2995 & 0.0 & 0.0 & 0.0 & 0.0 & 0.0 & 0.0 & 0.0 & 0.0 & 0.0 & 0.0 \\
\hline 3000 & 0.6 & 0.0 & 1.1 & 0.1 & 1.9 & 0.1 & 0.9 & 0.0 & 0.6 & 0.1 \\
\hline 3030 & 0.3 & 0.1 & 0.1 & 0.1 & 0.1 & 0.0 & 0.1 & 0.1 & 0.0 & 0.0 \\
\hline 3046 & 0.0 & 0.0 & 0.0 & 0.0 & 0.2 & 0.0 & 0.0 & 0.0 & 0.1 & 0.1 \\
\hline 3060 & 0.4 & 0.0 & 0.5 & 0.2 & 0.8 & 0.1 & 0.0 & 0.0 & 0.4 & 0.0 \\
\hline 3074 & 0.2 & 0.0 & 0.0 & 0.0 & 0.0 & 0.0 & 0.3 & 0.0 & 0.6 & 0.0 \\
\hline 3093 & 0.3 & 0.0 & 0.0 & 0.0 & 0.0 & 0.0 & 0.1 & 0.0 & 0.0 & 0.0 \\
\hline 3100 & 5.1 & 0.3 & 7.6 & 0.6 & 7.0 & 0.6 & 6.8 & 0.4 & 3.6 & 0.1 \\
\hline 3130 & 0.0 & 0.0 & 0.0 & 0.0 & 0.1 & 0.1 & 0.2 & 0.0 & 0.1 & 0.1 \\
\hline 3135 & 0.0 & 0.0 & 0.0 & 0.0 & 0.0 & 0.0 & 0.0 & 0.0 & 0.0 & 0.0 \\
\hline 3140 & 0.1 & 0.0 & 0.1 & 0.1 & 0.1 & 0.1 & 0.1 & 0.0 & 0.1 & 0.1 \\
\hline 3150 & 0.1 & 0.0 & 0.2 & 0.1 & 0.2 & 0.1 & 0.0 & 0.0 & 0.1 & 0.0 \\
\hline 3160 & 0.0 & 0.0 & 0.0 & 0.0 & 0.0 & 0.0 & 0.0 & 0.0 & 0.0 & 0.0 \\
\hline 3175 & 4.7 & 0.5 & 0.9 & 0.2 & 0.9 & 0.1 & 11.4 & 1.2 & 13.7 & 0.6 \\
\hline 3187 & 3.1 & 0.4 & 0.1 & 0.1 & 0.0 & 0.0 & 1.4 & 0.2 & 0.0 & 0.0 \\
\hline 3192 & 0.0 & 0.0 & 0.0 & 0.0 & 0.0 & 0.0 & 0.0 & 0.0 & 0.0 & 0.0 \\
\hline 3200 & 0.4 & 0.0 & 0.7 & 0.1 & 0.7 & 0.1 & 0.4 & 0.0 & 0.2 & 0.0 \\
\hline 3206 & 0.1 & 0.0 & 0.0 & 0.0 & 0.0 & 0.0 & 0.2 & 0.0 & 0.5 & 0.1 \\
\hline 3212 & 0.0 & 0.0 & 0.0 & 0.0 & 0.0 & 0.0 & 0.0 & 0.0 & 0.0 & 0.0 \\
\hline 3242 & 0.7 & 0.3 & 0.6 & 0.2 & 0.8 & 0.2 & 0.3 & 0.2 & 0.3 & 0.1 \\
\hline 3257 & 0.8 & 0.1 & 0.4 & 0.1 & 1.8 & 0.3 & 1.4 & 0.2 & 1.0 & 0.1 \\
\hline 3274 & 0.2 & 0.0 & 0.2 & 0.1 & 0.1 & 0.1 & 0.4 & 0.0 & 0.3 & 0.0 \\
\hline 3300 & 4.3 & 0.3 & 5.0 & 0.7 & 4.4 & 0.3 & 3.9 & 0.3 & 2.0 & 0.2 \\
\hline 3330 & 0.1 & 0.0 & 0.0 & 0.0 & 0.0 & 0.0 & 0.1 & 0.0 & 0.1 & 0.0 \\
\hline 3341 & 0.0 & 0.0 & 0.0 & 0.0 & 0.0 & 0.0 & 0.0 & 0.0 & 0.0 & 0.0 \\
\hline 3351 & 0.2 & 0.0 & 0.1 & 0.1 & 0.2 & 0.1 & 0.1 & 0.1 & 0.1 & 0.1 \\
\hline 3357 & 0.0 & 0.0 & 0.2 & 0.1 & 0.2 & 0.1 & 0.0 & 0.0 & 0.1 & 0.0 \\
\hline 3372 & 2.0 & 0.2 & 3.3 & 0.5 & 0.7 & 0.1 & 2.8 & 0.2 & 1.6 & 0.2 \\
\hline 3378 & 2.3 & 0.2 & 0.4 & 0.1 & 0.0 & 0.0 & 1.1 & 0.2 & 0.3 & 0.0 \\
\hline 3400 & 0.3 & 0.0 & 0.6 & 0.1 & 0.4 & 0.1 & 0.4 & 0.0 & 0.2 & 0.0 \\
\hline 3404 & 0.2 & 0.0 & 0.3 & 0.1 & 0.1 & 0.1 & 0.1 & 0.0 & 0.4 & 0.1 \\
\hline 3408 & 0.0 & 0.0 & 0.0 & 0.0 & 0.0 & 0.0 & 0.0 & 0.0 & 0.0 & 0.0 \\
\hline 3425 & 0.0 & 0.0 & 0.1 & 0.1 & 0.0 & 0.0 & 0.0 & 0.0 & 0.4 & 0.1 \\
\hline 3445 & 1.0 & 0.7 & 0.6 & 0.2 & 0.8 & 0.3 & 0.3 & 0.2 & 0.3 & 0.1 \\
\hline 3460 & 0.3 & 0.0 & 1.8 & 0.4 & 0.7 & 0.1 & 0.4 & 0.0 & 0.2 & 0.0 \\
\hline 3472 & 0.1 & 0.0 & 0.1 & 0.1 & 0.0 & 0.0 & 0.0 & 0.0 & 0.3 & 0.0 \\
\hline
\end{tabular}




\begin{tabular}{|c|c|c|c|c|c|c|c|c|c|c|}
\hline \multirow[b]{2}{*}{ KI } & \multicolumn{2}{|c|}{ Formosa } & \multicolumn{2}{|c|}{ Apolo } & \multicolumn{2}{|c|}{ Izozog G1 } & \multicolumn{2}{|c|}{ Izozog G2 } & \multicolumn{2}{|c|}{ Quillacollo } \\
\hline & media & $\overline{\mathrm{es}}$ & media & $\overline{\mathrm{es}}$ & media & $\overline{\mathrm{es}}$ & media & $\overline{\mathrm{es}}$ & media & $\overline{\mathrm{es}}$ \\
\hline 3480 & 0.2 & 0.0 & 0.1 & 0.0 & 0.0 & 0.0 & 0.1 & 0.0 & 0.3 & 0.0 \\
\hline 3500 & 0.1 & 0.0 & 0.4 & 0.2 & 0.2 & 0.1 & 0.1 & 0.1 & 0.0 & 0.0 \\
\hline 3527 & 0.7 & 0.0 & 0.9 & 0.1 & 0.6 & 0.1 & 0.5 & 0.1 & 1.2 & 0.1 \\
\hline 3548 & 0.0 & 0.0 & 0.6 & 0.1 & 0.0 & 0.0 & 0.0 & 0.0 & 0.0 & 0.0 \\
\hline 3554 & 0.1 & 0.0 & 0.5 & 0.1 & 0.3 & 0.0 & 0.1 & 0.1 & 0.2 & 0.1 \\
\hline 3575 & 0.7 & 0.1 & 2.9 & 0.3 & 0.6 & 0.1 & 0.7 & 0.1 & 2.4 & 0.2 \\
\hline 3625 & 0.3 & 0.0 & 0.6 & 0.1 & 0.1 & 0.1 & 0.1 & 0.0 & 0.9 & 0.2 \\
\hline 3650 & 0.5 & 0.4 & 0.5 & 0.1 & 0.2 & 0.2 & 0.1 & 0.1 & 0.1 & 0.1 \\
\hline 3654 & 1.7 & 0.2 & 0.3 & 0.1 & 0.6 & 0.1 & 2.1 & 0.3 & 0.2 & 0.1 \\
\hline 3670 & 0.9 & 0.1 & 1.6 & 0.2 & 0.8 & 0.2 & 0.7 & 0.1 & 2.4 & 0.5 \\
\hline 3680 & 0.0 & 0.0 & 0.0 & 0.0 & 0.0 & 0.0 & 0.0 & 0.0 & 0.0 & 0.0 \\
\hline 3725 & 3.7 & 0.3 & 3.5 & 0.2 & 3.7 & 0.5 & 3.4 & 0.2 & 5.5 & 0.3 \\
\hline 3750 & 0.7 & 0.1 & 2.6 & 0.6 & 1.1 & 0.2 & 1.1 & 0.3 & 1.3 & 0.1 \\
\hline 3766 & 0.1 & 0.0 & 0.2 & 0.1 & 0.3 & 0.1 & 0.1 & 0.0 & 0.9 & 0.1 \\
\hline 3773 & 3.7 & 0.3 & 6.3 & 0.5 & 3.7 & 0.4 & 3.8 & 0.2 & 8.0 & 0.9 \\
\hline 3800 & 1.9 & 0.2 & 2.2 & 0.2 & 0.9 & 0.1 & 1.9 & 0.2 & 2.5 & 0.3 \\
\hline 3823 & 0.3 & 0.0 & 0.8 & 0.1 & 1.2 & 0.1 & 0.5 & 0.1 & 2.1 & 0.2 \\
\hline 3830 & 0.3 & 0.0 & 0.1 & 0.0 & 0.0 & 0.0 & 0.2 & 0.0 & 0.5 & 0.1 \\
\hline 3844 & 0.2 & 0.1 & 0.4 & 0.0 & 0.1 & 0.1 & 0.2 & 0.1 & 0.4 & 0.0 \\
\hline 3857 & 0.0 & 0.0 & 0.0 & 0.0 & 0.0 & 0.0 & 0.0 & 0.0 & 0.0 & 0.0 \\
\hline 3866 & 1.3 & 0.2 & 1.7 & 0.2 & 1.4 & 0.2 & 1.5 & 0.1 & 3.3 & 0.4 \\
\hline 3877 & 0.4 & 0.1 & 0.2 & 0.1 & 0.3 & 0.0 & 0.4 & 0.1 & 0.3 & 0.0 \\
\hline 3920 & 1.0 & 0.1 & 1.0 & 0.1 & 1.7 & 0.2 & 1.0 & 0.2 & 1.4 & 0.0 \\
\hline 3941 & 0.3 & 0.0 & 0.6 & 0.1 & 0.8 & 0.1 & 0.6 & 0.1 & 0.7 & 0.0 \\
\hline 3965 & 1.7 & 0.2 & 1.8 & 0.2 & 2.9 & 0.2 & 1.9 & 0.1 & 4.0 & 0.3 \\
\hline 3971 & 0.0 & 0.0 & 0.0 & 0.0 & 0.0 & 0.0 & 0.0 & 0.0 & 0.0 & 0.0 \\
\hline 4003 & 0.7 & 0.1 & 0.5 & 0.1 & 0.4 & 0.0 & 0.9 & 0.1 & 1.1 & 0.2 \\
\hline 4046 & 0.0 & 0.0 & 0.0 & 0.0 & 0.0 & 0.0 & 0.0 & 0.0 & 0.1 & 0.0 \\
\hline 4125 & 0.0 & 0.0 & 0.0 & 0.0 & 0.0 & 0.0 & 0.0 & 0.0 & 0.0 & 0.0 \\
\hline
\end{tabular}

\begin{tabular}{|c|c|c|c|c|c|c|c|c|c|c|}
\hline \multirow[b]{2}{*}{ KI } & \multicolumn{2}{|c|}{ Goias } & \multicolumn{2}{|c|}{ Rioverde } & \multicolumn{2}{|c|}{ Rondonopolis } & \multicolumn{2}{|c|}{ Tocantins } & \multicolumn{2}{|c|}{ Boqueron } \\
\hline & media & es & media & es & media & $\overline{\text { es }}$ & media & es & media & es \\
\hline 2100 & 0.0 & 0.0 & 0.2 & 0.1 & 0.1 & 0.0 & 0.0 & 0.0 & 0.0 & 0.0 \\
\hline 2200 & 0.0 & 0.0 & 0.4 & 0.1 & 0.2 & 0.1 & 0.2 & 0.1 & 0.1 & 0.0 \\
\hline 2300 & 0.0 & 0.0 & 0.2 & 0.0 & 0.1 & 0.0 & 0.1 & 0.0 & 0.2 & 0.1 \\
\hline 2330 & 0.0 & 0.0 & 0.0 & 0.0 & 0.0 & 0.0 & 0.0 & 0.0 & 0.0 & 0.0 \\
\hline 2392 & 0.0 & 0.0 & 0.0 & 0.0 & 0.0 & 0.0 & 0.0 & 0.0 & 0.0 & 0.0 \\
\hline 2400 & 0.0 & 0.0 & 0.3 & 0.0 & 0.3 & 0.0 & 0.2 & 0.0 & 0.2 & 0.1 \\
\hline 2435 & 0.0 & 0.0 & 0.0 & 0.0 & 0.0 & 0.0 & 0.0 & 0.0 & 0.0 & 0.0 \\
\hline 2460 & 0.0 & 0.0 & 0.0 & 0.0 & 0.0 & 0.0 & 0.0 & 0.0 & 0.1 & 0.0 \\
\hline 2500 & 0.6 & 0.2 & 1.2 & 0.2 & 2.2 & 0.5 & 1.8 & 0.5 & 1.3 & 0.2 \\
\hline 2532 & 0.2 & 0.0 & 0.4 & 0.1 & 0.1 & 0.0 & 0.1 & 0.0 & 0.2 & 0.1 \\
\hline 2570 & 0.0 & 0.0 & 0.0 & 0.0 & 0.0 & 0.0 & 0.0 & 0.0 & 0.0 & 0.0 \\
\hline 2600 & 0.2 & 0.1 & 0.7 & 0.1 & 0.5 & 0.1 & 0.6 & 0.1 & 0.6 & 0.1 \\
\hline 2605 & 0.0 & 0.0 & 0.0 & 0.0 & 0.0 & 0.0 & 0.0 & 0.0 & 0.0 & 0.0 \\
\hline 2632 & 0.0 & 0.0 & 0.0 & 0.0 & 0.1 & 0.1 & 0.0 & 0.0 & 0.0 & 0.0 \\
\hline
\end{tabular}




\begin{tabular}{|c|c|c|c|c|c|c|c|c|c|c|}
\hline \multirow[b]{2}{*}{$\mathrm{KI}$} & \multicolumn{2}{|c|}{ Goias } & \multicolumn{2}{|c|}{ Rioverde } & \multicolumn{2}{|c|}{ Rondonopolis } & \multicolumn{2}{|c|}{ Tocantins } & \multicolumn{2}{|c|}{ Boqueron } \\
\hline & media & es & media & es & media & es & media & es & media & es \\
\hline 2662 & 0.1 & 0.1 & 0.0 & 0.0 & 0.1 & 0.0 & 0.2 & 0.1 & 0.8 & 0.1 \\
\hline 2672 & 0.0 & 0.0 & 0.0 & 0.0 & 0.0 & 0.0 & 0.0 & 0.0 & 0.0 & 0.0 \\
\hline 2700 & 4.6 & 0.6 & 8.0 & 0.8 & 8.0 & 0.9 & 9.7 & 1.0 & 6.1 & 0.7 \\
\hline 2732 & 0.2 & 0.1 & 0.1 & 0.0 & 0.4 & 0.1 & 0.2 & 0.0 & 0.1 & 0.0 \\
\hline 2750 & 0.0 & 0.0 & 0.0 & 0.0 & 0.0 & 0.0 & 0.0 & 0.0 & 0.0 & 0.0 \\
\hline 2760 & 0.0 & 0.0 & 0.0 & 0.0 & 0.0 & 0.0 & 0.0 & 0.0 & 0.0 & 0.0 \\
\hline 2772 & 0.1 & 0.0 & 0.2 & 0.0 & 0.2 & 0.1 & 0.2 & 0.0 & 0.6 & 0.2 \\
\hline 2780 & 0.0 & 0.0 & 0.0 & 0.0 & 0.0 & 0.0 & 0.0 & 0.0 & 0.0 & 0.0 \\
\hline 2800 & 5.3 & 0.8 & 2.6 & 0.1 & 2.6 & 0.2 & 2.0 & 0.2 & 2.5 & 0.1 \\
\hline 2805 & 0.0 & 0.0 & 0.0 & 0.0 & 0.0 & 0.0 & 0.0 & 0.0 & 0.0 & 0.0 \\
\hline 2831 & 0.0 & 0.0 & 0.1 & 0.0 & 0.1 & 0.0 & 0.1 & 0.0 & 0.4 & 0.2 \\
\hline 2844 & 0.0 & 0.0 & 0.0 & 0.0 & 0.0 & 0.0 & 0.0 & 0.0 & 0.0 & 0.0 \\
\hline 2857 & 0.2 & 0.0 & 0.2 & 0.1 & 0.8 & 0.2 & 0.1 & 0.0 & 0.1 & 0.0 \\
\hline 2861 & 0.0 & 0.0 & 0.0 & 0.0 & 0.0 & 0.0 & 0.0 & 0.0 & 0.2 & 0.0 \\
\hline 2905 & 22.2 & 2.8 & 18.3 & 1.2 & 20.8 & 1.9 & 16.7 & 1.4 & 25.0 & 1.4 \\
\hline 2932 & 0.2 & 0.0 & 0.4 & 0.1 & 0.6 & 0.2 & 0.7 & 0.1 & 0.3 & 0.1 \\
\hline 2941 & 0.0 & 0.0 & 0.0 & 0.0 & 0.1 & 0.0 & 0.1 & 0.0 & 0.1 & 0.0 \\
\hline 2951 & 0.3 & 0.1 & 0.1 & 0.0 & 0.3 & 0.0 & 0.5 & 0.0 & 0.1 & 0.0 \\
\hline 2958 & 0.0 & 0.0 & 0.2 & 0.1 & 0.2 & 0.0 & 0.2 & 0.0 & 0.1 & 0.0 \\
\hline 2974 & 0.9 & 0.3 & 0.7 & 0.1 & 1.2 & 0.2 & 0.4 & 0.1 & 1.7 & 0.4 \\
\hline 2982 & 0.0 & 0.0 & 0.0 & 0.0 & 0.1 & 0.0 & 0.1 & 0.0 & 0.0 & 0.0 \\
\hline 2995 & 0.0 & 0.0 & 0.0 & 0.0 & 0.1 & 0.0 & 0.0 & 0.0 & 0.0 & 0.0 \\
\hline 3000 & 1.0 & 0.0 & 1.1 & 0.1 & 0.8 & 0.1 & 0.8 & 0.0 & 1.4 & 0.1 \\
\hline 3030 & 0.3 & 0.1 & 0.0 & 0.0 & 0.1 & 0.1 & 0.0 & 0.0 & 0.0 & 0.0 \\
\hline 3046 & 0.0 & 0.0 & 0.0 & 0.0 & 0.1 & 0.1 & 0.0 & 0.0 & 0.1 & 0.0 \\
\hline 3060 & 0.6 & 0.1 & 0.2 & 0.1 & 1.2 & 0.2 & 0.2 & 0.1 & 0.2 & 0.1 \\
\hline 3074 & 0.0 & 0.0 & 0.0 & 0.0 & 0.0 & 0.0 & 0.0 & 0.0 & 0.2 & 0.0 \\
\hline 3093 & 0.0 & 0.0 & 0.2 & 0.1 & 0.1 & 0.1 & 0.2 & 0.1 & 0.1 & 0.0 \\
\hline 3100 & 8.5 & 0.5 & 8.6 & 1.1 & 7.9 & 0.6 & 8.0 & 0.6 & 11.0 & 1.0 \\
\hline 3130 & 0.1 & 0.0 & 0.1 & 0.0 & 0.2 & 0.0 & 0.3 & 0.1 & 0.3 & 0.0 \\
\hline 3135 & 0.0 & 0.0 & 0.0 & 0.0 & 0.0 & 0.0 & 0.0 & 0.0 & 0.0 & 0.0 \\
\hline 3140 & 0.1 & 0.0 & 0.0 & 0.0 & 0.1 & 0.0 & 0.1 & 0.0 & 0.2 & 0.0 \\
\hline 3150 & 0.0 & 0.0 & 0.1 & 0.1 & 0.2 & 0.1 & 0.5 & 0.1 & 0.2 & 0.0 \\
\hline 3160 & 0.0 & 0.0 & 0.0 & 0.0 & 0.0 & 0.0 & 0.0 & 0.0 & 0.0 & 0.0 \\
\hline 3175 & 1.2 & 0.2 & 0.5 & 0.1 & 0.5 & 0.1 & 0.8 & 0.1 & 5.5 & 0.8 \\
\hline 3187 & 0.0 & 0.0 & 0.0 & 0.0 & 0.0 & 0.0 & 0.0 & 0.0 & 1.1 & 0.3 \\
\hline 3192 & 0.0 & 0.0 & 0.0 & 0.0 & 0.0 & 0.0 & 0.0 & 0.0 & 0.0 & 0.0 \\
\hline 3200 & 0.6 & 0.1 & 1.0 & 0.0 & 1.2 & 0.2 & 1.4 & 0.1 & 0.6 & 0.0 \\
\hline 3206 & 0.0 & 0.0 & 0.0 & 0.0 & 0.0 & 0.0 & 0.0 & 0.0 & 0.1 & 0.0 \\
\hline 3212 & 0.0 & 0.0 & 0.0 & 0.0 & 0.0 & 0.0 & 0.0 & 0.0 & 0.0 & 0.0 \\
\hline 3242 & 0.9 & 0.4 & 0.3 & 0.1 & 1.6 & 0.7 & 0.3 & 0.1 & 0.1 & 0.0 \\
\hline 3257 & 0.7 & 0.1 & 0.2 & 0.0 & 0.6 & 0.1 & 0.2 & 0.0 & 1.6 & 0.2 \\
\hline 3274 & 0.3 & 0.0 & 0.0 & 0.0 & 0.1 & 0.0 & 0.1 & 0.0 & 0.2 & 0.0 \\
\hline
\end{tabular}




\begin{tabular}{|c|c|c|c|c|c|c|c|c|c|c|}
\hline \multirow[b]{2}{*}{$\mathrm{KI}$} & \multicolumn{2}{|c|}{ Goias } & \multicolumn{2}{|c|}{ Rioverde } & \multicolumn{2}{|c|}{ Rondonopolis } & \multicolumn{2}{|c|}{ Tocantins } & \multicolumn{2}{|c|}{ Boqueron } \\
\hline & media & $\overline{\mathrm{es}}$ & media & $\overline{\mathrm{es}}$ & media & $\overline{\mathrm{es}}$ & media & $\overline{\text { es }}$ & media & es \\
\hline 3300 & 5.2 & 0.4 & 6.5 & 0.7 & 4.0 & 0.3 & 5.7 & 0.5 & 6.4 & 0.7 \\
\hline 3330 & 0.5 & 0.1 & 0.0 & 0.0 & 0.4 & 0.1 & 0.3 & 0.1 & 0.3 & 0.0 \\
\hline 3341 & 0.0 & 0.0 & 0.0 & 0.0 & 0.0 & 0.0 & 0.0 & 0.0 & 0.0 & 0.0 \\
\hline 3351 & 0.1 & 0.0 & 0.1 & 0.0 & 0.3 & 0.1 & 0.7 & 0.1 & 0.9 & 0.1 \\
\hline 3357 & 0.5 & 0.0 & 0.0 & 0.0 & 0.5 & 0.1 & 0.2 & 0.0 & 0.2 & 0.0 \\
\hline 3372 & 3.9 & 0.2 & 1.1 & 0.2 & 1.1 & 0.2 & 1.1 & 0.1 & 2.5 & 0.4 \\
\hline 3378 & 0.1 & 0.1 & 0.4 & 0.1 & 0.9 & 0.1 & 0.3 & 0.1 & 4.0 & 0.3 \\
\hline 3400 & 0.9 & 0.3 & 0.7 & 0.1 & 1.1 & 0.2 & 1.0 & 0.1 & 0.4 & 0.0 \\
\hline 3404 & 0.3 & 0.0 & 0.0 & 0.0 & 0.1 & 0.0 & 0.0 & 0.0 & 0.0 & 0.0 \\
\hline 3408 & 0.0 & 0.0 & 0.0 & 0.0 & 0.0 & 0.0 & 0.0 & 0.0 & 0.0 & 0.0 \\
\hline 3425 & 0.3 & 0.1 & 1.9 & 0.6 & 1.3 & 0.9 & 1.9 & 0.4 & 0.1 & 0.0 \\
\hline 3445 & 1.0 & 0.5 & 0.4 & 0.1 & 1.9 & 0.8 & 0.4 & 0.1 & 0.2 & 0.1 \\
\hline 3460 & 3.7 & 0.8 & 0.7 & 0.1 & 1.4 & 0.1 & 0.7 & 0.1 & 0.5 & 0.1 \\
\hline 3472 & 0.3 & 0.1 & 0.1 & 0.0 & 0.1 & 0.0 & 0.4 & 0.1 & 0.0 & 0.0 \\
\hline 3480 & 0.0 & 0.0 & 0.2 & 0.1 & 0.5 & 0.2 & 0.0 & 0.0 & 0.5 & 0.1 \\
\hline 3500 & 0.7 & 0.1 & 0.8 & 0.2 & 0.2 & 0.1 & 0.7 & 0.2 & 0.1 & 0.0 \\
\hline 3527 & 1.1 & 0.2 & 0.7 & 0.0 & 1.1 & 0.1 & 1.0 & 0.1 & 0.2 & 0.0 \\
\hline 3548 & 1.0 & 0.2 & 0.4 & 0.1 & 0.6 & 0.2 & 0.9 & 0.1 & 0.1 & 0.0 \\
\hline 3554 & 0.7 & 0.1 & 0.4 & 0.0 & 0.8 & 0.2 & 0.7 & 0.1 & 0.6 & 0.1 \\
\hline 3575 & 2.8 & 0.2 & 2.7 & 0.4 & 3.0 & 0.3 & 3.1 & 0.2 & 0.8 & 0.1 \\
\hline 3625 & 0.5 & 0.0 & 0.1 & 0.0 & 0.3 & 0.1 & 0.4 & 0.1 & 0.4 & 0.0 \\
\hline 3650 & 0.5 & 0.2 & 0.3 & 0.1 & 0.7 & 0.2 & 0.2 & 0.1 & 0.1 & 0.1 \\
\hline 3654 & 2.2 & 0.7 & 0.9 & 0.2 & 0.7 & 0.2 & 0.7 & 0.1 & 1.5 & 0.1 \\
\hline 3670 & 1.2 & 0.2 & 1.7 & 0.2 & 1.5 & 0.1 & 1.9 & 0.2 & 0.4 & 0.1 \\
\hline 3680 & 0.0 & 0.0 & 0.0 & 0.0 & 0.0 & 0.0 & 0.0 & 0.0 & 0.0 & 0.0 \\
\hline 3725 & 4.5 & 0.5 & 4.6 & 0.3 & 3.8 & 0.4 & 4.3 & 0.2 & 1.5 & 0.1 \\
\hline 3750 & 1.7 & 0.2 & 3.1 & 0.4 & 2.3 & 0.4 & 3.4 & 0.4 & 1.1 & 0.1 \\
\hline 3766 & 0.0 & 0.0 & 0.3 & 0.1 & 0.1 & 0.1 & 0.2 & 0.1 & 0.0 & 0.0 \\
\hline 3773 & 6.0 & 0.6 & 8.3 & 1.0 & 6.9 & 0.4 & 6.0 & 0.5 & 3.2 & 0.6 \\
\hline 3800 & 2.9 & 0.1 & 2.7 & 0.3 & 2.1 & 0.1 & 2.1 & 0.1 & 1.7 & 0.2 \\
\hline 3823 & 0.9 & 0.1 & 0.7 & 0.1 & 0.7 & 0.1 & 0.8 & 0.1 & 0.8 & 0.1 \\
\hline 3830 & 0.1 & 0.1 & 0.3 & 0.1 & 0.0 & 0.0 & 0.3 & 0.1 & 0.0 & 0.0 \\
\hline 3844 & 0.3 & 0.0 & 0.6 & 0.1 & 0.6 & 0.0 & 0.8 & 0.1 & 0.4 & 0.0 \\
\hline 3857 & 0.0 & 0.0 & 0.0 & 0.0 & 0.0 & 0.0 & 0.0 & 0.0 & 0.0 & 0.0 \\
\hline 3866 & 1.4 & 0.1 & 2.7 & 0.4 & 1.9 & 0.1 & 2.5 & 0.3 & 1.4 & 0.2 \\
\hline 3877 & 0.5 & 0.1 & 0.2 & 0.0 & 0.2 & 0.1 & 0.2 & 0.1 & 0.2 & 0.1 \\
\hline 3920 & 1.2 & 0.1 & 1.9 & 0.2 & 1.0 & 0.2 & 2.3 & 0.4 & 1.4 & 0.1 \\
\hline 3941 & 0.5 & 0.1 & 1.3 & 0.2 & 0.9 & 0.1 & 2.1 & 0.3 & 1.2 & 0.1 \\
\hline 3965 & 2.0 & 0.2 & 3.3 & 0.4 & 2.2 & 0.4 & 3.1 & 0.4 & 2.3 & 0.4 \\
\hline 3971 & 0.0 & 0.0 & 0.0 & 0.0 & 0.0 & 0.0 & 0.0 & 0.0 & 0.0 & 0.0 \\
\hline 4003 & 0.6 & 0.1 & 1.0 & 0.1 & 0.5 & 0.1 & 0.9 & 0.1 & 1.0 & 0.1 \\
\hline 4046 & 0.0 & 0.0 & 0.0 & 0.0 & 0.0 & 0.0 & 0.0 & 0.0 & 0.1 & 0.0 \\
\hline 4125 & 0.0 & 0.0 & 0.0 & 0.0 & 0.0 & 0.0 & 0.0 & 0.0 & 0.0 & 0.0 \\
\hline
\end{tabular}




\begin{tabular}{|c|c|c|c|c|c|c|c|c|c|c|}
\hline \multirow[b]{2}{*}{$\mathrm{KI}$} & \multicolumn{2}{|c|}{ Concepcion } & \multicolumn{2}{|c|}{ Gaira } & \multicolumn{2}{|c|}{ Paraguari } & \multicolumn{2}{|c|}{ PteHayes } & \multicolumn{2}{|c|}{ SanPedro } \\
\hline & media & es & media & $\overline{\mathrm{es}}$ & media & $\overline{\mathrm{es}}$ & media & $\overline{\mathrm{es}}$ & media & $\overline{\mathrm{es}}$ \\
\hline 2100 & 0.0 & 0.0 & 0.0 & 0.0 & 0.1 & 0.0 & 0.1 & 0.0 & 0.0 & 0.0 \\
\hline 2200 & 0.1 & 0.0 & 0.0 & 0.0 & 0.2 & 0.1 & 0.3 & 0.2 & 0.1 & 0.0 \\
\hline 2300 & 0.3 & 0.1 & 0.2 & 0.0 & 0.2 & 0.1 & 0.3 & 0.1 & 0.2 & 0.1 \\
\hline 2330 & 0.0 & 0.0 & 0.0 & 0.0 & 0.0 & 0.0 & 0.0 & 0.0 & 0.0 & 0.0 \\
\hline 2392 & 0.0 & 0.0 & 0.0 & 0.0 & 0.0 & 0.0 & 0.0 & 0.0 & 0.0 & 0.0 \\
\hline 2400 & 0.2 & 0.1 & 0.1 & 0.0 & 0.2 & 0.1 & 0.3 & 0.1 & 0.2 & 0.0 \\
\hline 2435 & 0.0 & 0.0 & 0.1 & 0.0 & 0.1 & 0.1 & 0.1 & 0.0 & 0.0 & 0.0 \\
\hline 2460 & 0.0 & 0.0 & 0.0 & 0.0 & 0.0 & 0.0 & 0.1 & 0.0 & 0.0 & 0.0 \\
\hline 2500 & 3.4 & 0.6 & 3.0 & 1.0 & 1.4 & 0.7 & 1.4 & 0.3 & 1.8 & 0.5 \\
\hline 2532 & 0.1 & 0.0 & 0.1 & 0.0 & 0.2 & 0.1 & 0.3 & 0.1 & 0.1 & 0.0 \\
\hline 2570 & 0.1 & 0.0 & 0.0 & 0.0 & 0.0 & 0.0 & 0.0 & 0.0 & 0.0 & 0.0 \\
\hline 2600 & 0.5 & 0.1 & 1.1 & 0.2 & 0.4 & 0.2 & 0.5 & 0.1 & 0.5 & 0.1 \\
\hline 2605 & 0.0 & 0.0 & 0.0 & 0.0 & 0.0 & 0.0 & 0.0 & 0.0 & 0.0 & 0.0 \\
\hline 2632 & 0.0 & 0.0 & 0.0 & 0.0 & 0.0 & 0.0 & 0.0 & 0.0 & 0.0 & 0.0 \\
\hline 2662 & 0.1 & 0.0 & 0.0 & 0.0 & 0.1 & 0.1 & 0.6 & 0.1 & 0.1 & 0.0 \\
\hline 2672 & 0.0 & 0.0 & 0.0 & 0.0 & 0.0 & 0.0 & 0.0 & 0.0 & 0.0 & 0.0 \\
\hline 2700 & 12.2 & 1.7 & 22.7 & 3.4 & 9.7 & 2.8 & 6.1 & 0.6 & 13.1 & 1.2 \\
\hline 2732 & 0.6 & 0.2 & 0.3 & 0.2 & 0.4 & 0.2 & 0.1 & 0.1 & 0.2 & 0.1 \\
\hline 2750 & 0.0 & 0.0 & 0.0 & 0.0 & 0.0 & 0.0 & 0.0 & 0.0 & 0.0 & 0.0 \\
\hline 2760 & 0.0 & 0.0 & 0.0 & 0.0 & 0.0 & 0.0 & 0.0 & 0.0 & 0.0 & 0.0 \\
\hline 2772 & 1.7 & 0.4 & 2.3 & 0.1 & 1.5 & 0.5 & 0.8 & 0.2 & 1.2 & 0.3 \\
\hline 2780 & 0.0 & 0.0 & 0.0 & 0.0 & 0.0 & 0.0 & 0.0 & 0.0 & 0.0 & 0.0 \\
\hline 2800 & 2.1 & 0.2 & 2.8 & 0.7 & 2.8 & 0.3 & 2.7 & 0.2 & 3.4 & 0.3 \\
\hline 2805 & 0.0 & 0.0 & 0.0 & 0.0 & 0.0 & 0.0 & 0.0 & 0.0 & 0.0 & 0.0 \\
\hline 2831 & 0.1 & 0.1 & 0.0 & 0.0 & 0.4 & 0.3 & 0.7 & 0.4 & 0.5 & 0.3 \\
\hline 2844 & 0.0 & 0.0 & 0.0 & 0.0 & 0.1 & 0.1 & 0.1 & 0.1 & 0.0 & 0.0 \\
\hline 2857 & 0.3 & 0.1 & 0.5 & 0.1 & 0.1 & 0.1 & 0.0 & 0.0 & 0.3 & 0.0 \\
\hline 2861 & 0.0 & 0.0 & 0.0 & 0.0 & 0.0 & 0.0 & 0.4 & 0.2 & 0.0 & 0.0 \\
\hline 2905 & 17.2 & 1.8 & 15.6 & 1.2 & 27.5 & 2.1 & 25.6 & 1.5 & 29.6 & 2.6 \\
\hline 2932 & 1.0 & 0.3 & 0.4 & 0.2 & 0.4 & 0.3 & 0.4 & 0.1 & 0.5 & 0.2 \\
\hline 2941 & 0.3 & 0.1 & 0.1 & 0.1 & 0.1 & 0.1 & 0.1 & 0.0 & 0.2 & 0.1 \\
\hline 2951 & 0.6 & 0.1 & 0.6 & 0.1 & 0.2 & 0.1 & 0.1 & 0.0 & 0.4 & 0.1 \\
\hline 2958 & 0.8 & 0.4 & 0.2 & 0.1 & 0.2 & 0.2 & 0.4 & 0.2 & 0.2 & 0.1 \\
\hline 2974 & 4.3 & 1.6 & 3.4 & 0.2 & 3.9 & 0.4 & 1.3 & 0.3 & 4.6 & 0.5 \\
\hline 2982 & 0.1 & 0.0 & 0.0 & 0.0 & 0.0 & 0.0 & 0.0 & 0.0 & 0.0 & 0.0 \\
\hline 2995 & 0.0 & 0.0 & 0.0 & 0.0 & 0.0 & 0.0 & 0.0 & 0.0 & 0.0 & 0.0 \\
\hline 3000 & 0.7 & 0.1 & 0.5 & 0.0 & 0.9 & 0.1 & 1.3 & 0.1 & 0.9 & 0.1 \\
\hline 3030 & 0.1 & 0.0 & 0.0 & 0.0 & 0.1 & 0.1 & 0.0 & 0.0 & 0.0 & 0.0 \\
\hline 3046 & 0.0 & 0.0 & 0.0 & 0.0 & 0.0 & 0.0 & 0.0 & 0.0 & 0.0 & 0.0 \\
\hline 3060 & 0.3 & 0.1 & 0.3 & 0.0 & 0.3 & 0.0 & 0.2 & 0.1 & 0.3 & 0.0 \\
\hline 3074 & 0.1 & 0.0 & 0.1 & 0.0 & 0.0 & 0.0 & 0.1 & 0.0 & 0.1 & 0.0 \\
\hline 3093 & 0.0 & 0.0 & 0.0 & 0.0 & 0.0 & 0.0 & 0.1 & 0.1 & 0.1 & 0.0 \\
\hline 3100 & 8.1 & 0.7 & 6.0 & 1.8 & 9.9 & 1.8 & 9.0 & 0.9 & 8.4 & 0.9 \\
\hline
\end{tabular}




\begin{tabular}{|c|c|c|c|c|c|c|c|c|c|c|}
\hline \multirow[b]{2}{*}{$\mathrm{KI}$} & \multicolumn{2}{|c|}{ Concepcion } & \multicolumn{2}{|c|}{ Gaira } & \multicolumn{2}{|c|}{ Paraguari } & \multicolumn{2}{|c|}{ PteHayes } & \multicolumn{2}{|c|}{ SanPedro } \\
\hline & media & es & media & es & media & es & media & es & media & es \\
\hline 3130 & 0.7 & 0.2 & 0.1 & 0.1 & 0.2 & 0.2 & 0.3 & 0.0 & 0.3 & 0.1 \\
\hline 3135 & 0.0 & 0.0 & 0.0 & 0.0 & 0.0 & 0.0 & 0.0 & 0.0 & 0.0 & 0.0 \\
\hline 3140 & 0.1 & 0.0 & 0.1 & 0.0 & 0.2 & 0.1 & 0.3 & 0.1 & 0.1 & 0.0 \\
\hline 3150 & 0.6 & 0.3 & 0.1 & 0.1 & 0.3 & 0.2 & 0.4 & 0.1 & 0.4 & 0.1 \\
\hline 3160 & 0.0 & 0.0 & 0.0 & 0.0 & 0.0 & 0.0 & 0.0 & 0.0 & 0.0 & 0.0 \\
\hline 3175 & 1.2 & 0.2 & 1.5 & 0.4 & 2.1 & 0.3 & 2.9 & 0.3 & 1.8 & 0.3 \\
\hline 3187 & 0.2 & 0.1 & 0.0 & 0.0 & 0.0 & 0.0 & 1.0 & 0.4 & 0.2 & 0.2 \\
\hline 3192 & 0.0 & 0.0 & 0.0 & 0.0 & 0.0 & 0.0 & 0.0 & 0.0 & 0.0 & 0.0 \\
\hline 3200 & 1.0 & 0.2 & 0.4 & 0.1 & 0.6 & 0.1 & 0.6 & 0.0 & 0.6 & 0.1 \\
\hline 3206 & 0.1 & 0.1 & 0.3 & 0.1 & 0.1 & 0.1 & 0.0 & 0.0 & 0.0 & 0.0 \\
\hline 3212 & 0.0 & 0.0 & 0.0 & 0.0 & 0.0 & 0.0 & 0.0 & 0.0 & 0.0 & 0.0 \\
\hline 3242 & 0.1 & 0.1 & 0.1 & 0.1 & 0.3 & 0.1 & 0.1 & 0.0 & 0.1 & 0.0 \\
\hline 3257 & 0.1 & 0.0 & 0.2 & 0.0 & 0.2 & 0.1 & 1.0 & 0.1 & 0.1 & 0.1 \\
\hline 3274 & 0.1 & 0.0 & 0.2 & 0.1 & 0.1 & 0.1 & 0.2 & 0.1 & 0.1 & 0.0 \\
\hline 3300 & 3.8 & 0.4 & 3.3 & 0.5 & 3.9 & 0.8 & 6.1 & 0.5 & 3.7 & 0.4 \\
\hline 3330 & 0.2 & 0.1 & 0.0 & 0.0 & 0.1 & 0.1 & 0.1 & 0.1 & 0.1 & 0.0 \\
\hline 3341 & 0.0 & 0.0 & 0.0 & 0.0 & 0.0 & 0.0 & 0.0 & 0.0 & 0.0 & 0.0 \\
\hline 3351 & 0.1 & 0.1 & 0.0 & 0.0 & 0.1 & 0.1 & 1.3 & 0.4 & 0.1 & 0.1 \\
\hline 3357 & 0.4 & 0.1 & 0.3 & 0.1 & 0.1 & 0.0 & 0.1 & 0.1 & 0.2 & 0.1 \\
\hline 3372 & 0.8 & 0.1 & 1.2 & 0.3 & 1.4 & 0.3 & 1.6 & 0.2 & 1.1 & 0.1 \\
\hline 3378 & 0.2 & 0.2 & 0.2 & 0.1 & 0.2 & 0.1 & 3.8 & 0.6 & 0.3 & 0.3 \\
\hline 3400 & 0.5 & 0.1 & 0.3 & 0.0 & 0.2 & 0.1 & 0.5 & 0.0 & 0.3 & 0.0 \\
\hline 3404 & 0.1 & 0.1 & 0.3 & 0.1 & 0.1 & 0.1 & 0.2 & 0.1 & 0.1 & 0.1 \\
\hline 3408 & 0.0 & 0.0 & 0.0 & 0.0 & 0.0 & 0.0 & 0.0 & 0.0 & 0.0 & 0.0 \\
\hline 3425 & 0.0 & 0.0 & 0.0 & 0.0 & 0.0 & 0.0 & 0.1 & 0.0 & 0.0 & 0.0 \\
\hline 3445 & 0.1 & 0.1 & 0.0 & 0.0 & 0.3 & 0.1 & 0.2 & 0.1 & 0.1 & 0.0 \\
\hline 3460 & 0.4 & 0.1 & 0.3 & 0.1 & 0.2 & 0.1 & 0.4 & 0.1 & 0.3 & 0.1 \\
\hline 3472 & 0.1 & 0.1 & 0.1 & 0.0 & 0.0 & 0.0 & 0.0 & 0.0 & 0.0 & 0.0 \\
\hline 3480 & 0.1 & 0.1 & 0.1 & 0.0 & 0.0 & 0.0 & 0.5 & 0.1 & 0.0 & 0.0 \\
\hline 3500 & 0.2 & 0.1 & 0.1 & 0.0 & 0.5 & 0.4 & 0.3 & 0.1 & 0.0 & 0.0 \\
\hline 3527 & 0.6 & 0.1 & 0.8 & 0.1 & 0.1 & 0.1 & 0.4 & 0.0 & 0.4 & 0.0 \\
\hline 3548 & 0.1 & 0.1 & 0.0 & 0.0 & 0.1 & 0.1 & 0.1 & 0.1 & 0.0 & 0.0 \\
\hline 3554 & 1.2 & 0.3 & 0.6 & 0.2 & 0.2 & 0.1 & 0.7 & 0.2 & 0.8 & 0.2 \\
\hline 3575 & 1.7 & 0.2 & 1.3 & 0.0 & 1.6 & 0.2 & 1.2 & 0.1 & 1.1 & 0.1 \\
\hline 3625 & 0.3 & 0.0 & 0.4 & 0.1 & 0.1 & 0.1 & 0.4 & 0.0 & 0.3 & 0.1 \\
\hline 3650 & 0.3 & 0.2 & 0.0 & 0.0 & 0.2 & 0.2 & 0.1 & 0.0 & 0.0 & 0.0 \\
\hline 3654 & 0.4 & 0.2 & 0.3 & 0.1 & 0.1 & 0.1 & 1.2 & 0.2 & 0.6 & 0.3 \\
\hline 3670 & 1.4 & 0.2 & 1.1 & 0.1 & 1.2 & 0.1 & 0.7 & 0.1 & 0.9 & 0.1 \\
\hline 3680 & 0.0 & 0.0 & 0.0 & 0.0 & 0.0 & 0.0 & 0.0 & 0.0 & 0.0 & 0.0 \\
\hline 3725 & 2.7 & 0.4 & 4.5 & 1.3 & 2.6 & 0.4 & 1.9 & 0.2 & 2.4 & 0.2 \\
\hline 3750 & 4.1 & 0.7 & 1.6 & 0.6 & 1.9 & 0.9 & 1.5 & 0.2 & 2.5 & 0.5 \\
\hline 3766 & 0.1 & 0.1 & 0.1 & 0.1 & 0.0 & 0.0 & 0.0 & 0.0 & 0.0 & 0.0 \\
\hline 3773 & 7.1 & 1.1 & 5.4 & 0.4 & 5.8 & 1.2 & 3.7 & 0.7 & 4.2 & 0.4 \\
\hline
\end{tabular}




\begin{tabular}{|c|c|c|c|c|c|c|c|c|c|c|}
\hline \multirow[b]{2}{*}{ KI } & \multicolumn{2}{|c|}{ Concepcion } & \multicolumn{2}{|c|}{ Gaira } & \multicolumn{2}{|c|}{ Paraguari } & \multicolumn{2}{|c|}{ PteHayes } & \multicolumn{2}{|c|}{ SanPedro } \\
\hline & media & $\overline{\text { es }}$ & media & $\overline{\text { es }}$ & media & $\overline{\text { es }}$ & media & $\overline{\text { es }}$ & media & es \\
\hline 3800 & 1.3 & 0.2 & 1.9 & 0.4 & 1.7 & 0.4 & 1.7 & 0.2 & 1.2 & 0.2 \\
\hline 3823 & 0.9 & 0.1 & 1.0 & 0.2 & 0.7 & 0.1 & 0.9 & 0.1 & 0.7 & 0.1 \\
\hline 3830 & 0.1 & 0.1 & 0.1 & 0.1 & 0.1 & 0.1 & 0.1 & 0.0 & 0.0 & 0.0 \\
\hline 3844 & 0.7 & 0.1 & 0.5 & 0.1 & 0.5 & 0.1 & 0.4 & 0.1 & 0.4 & 0.1 \\
\hline 3857 & 0.0 & 0.0 & 0.0 & 0.0 & 0.0 & 0.0 & 0.0 & 0.0 & 0.0 & 0.0 \\
\hline 3866 & 2.1 & 0.3 & 1.5 & 0.2 & 2.3 & 0.4 & 1.6 & 0.2 & 1.4 & 0.1 \\
\hline 3877 & 0.1 & 0.1 & 0.2 & 0.1 & 0.0 & 0.0 & 0.3 & 0.1 & 0.0 & 0.0 \\
\hline 3920 & 1.9 & 0.3 & 2.3 & 0.4 & 1.8 & 0.2 & 1.7 & 0.1 & 1.6 & 0.2 \\
\hline 3941 & 2.1 & 0.3 & 1.1 & 0.5 & 1.1 & 0.2 & 1.6 & 0.3 & 1.3 & 0.2 \\
\hline 3965 & 3.5 & 0.7 & 3.6 & 0.6 & 4.5 & 0.7 & 2.6 & 0.4 & 2.2 & 0.2 \\
\hline 3971 & 0.0 & 0.0 & 0.0 & 0.0 & 0.0 & 0.0 & 0.0 & 0.0 & 0.0 & 0.0 \\
\hline 4003 & 0.4 & 0.2 & 1.3 & 0.2 & 0.9 & 0.1 & 1.0 & 0.1 & 0.6 & 0.1 \\
\hline 4046 & 0.1 & 0.1 & 0.1 & 0.1 & 0.1 & 0.1 & 0.1 & 0.0 & 0.0 & 0.0 \\
\hline 4125 & 0.0 & 0.0 & 0.0 & 0.0 & 0.0 & 0.0 & 0.0 & 0.0 & 0.0 & 0.0 \\
\hline
\end{tabular}

es: error estándar o típico de la media

Tabla 6b. Composición de los HC cuticulares de T.guasayana.

\begin{tabular}{|c|c|c|c|c|c|c|c|c|}
\hline \multirow[b]{2}{*}{$\mathrm{KI}$} & \multicolumn{2}{|c|}{ SgoEstero } & \multicolumn{2}{|c|}{ Mataral } & \multicolumn{2}{|c|}{ Boqueron } & \multicolumn{2}{|c|}{ PteHayes } \\
\hline & media & es & media & es & media & es & media & es \\
\hline 2100 & 0.0 & 0.0 & 0.1 & 0.0 & 0.1 & 0.0 & 0.0 & 0.0 \\
\hline 2200 & 0.1 & 0.0 & 0.2 & 0.1 & 0.1 & 0.1 & 0.0 & 0.0 \\
\hline 2300 & 0.1 & 0.0 & 0.1 & 0.0 & 0.2 & 0.1 & 0.0 & 0.0 \\
\hline 2330 & 0.0 & 0.0 & 0.0 & 0.0 & 0.0 & 0.0 & 0.0 & 0.0 \\
\hline 2392 & 0.0 & 0.0 & 0.0 & 0.0 & 0.0 & 0.0 & 0.0 & 0.0 \\
\hline 2400 & 0.1 & 0.0 & 0.2 & 0.0 & 0.2 & 0.1 & 0.1 & 0.0 \\
\hline 2431 & 0.0 & 0.0 & 0.0 & 0.0 & 0.0 & 0.0 & 0.0 & 0.0 \\
\hline 2460 & 0.0 & 0.0 & 0.0 & 0.0 & 0.0 & 0.0 & 0.0 & 0.0 \\
\hline 2500 & 0.5 & 0.1 & 1.1 & 0.1 & 0.6 & 0.0 & 0.5 & 0.1 \\
\hline 2535 & 0.0 & 0.0 & 0.2 & 0.0 & 0.2 & 0.1 & 0.1 & 0.0 \\
\hline 2574 & 0.0 & 0.0 & 0.0 & 0.0 & 0.0 & 0.0 & 0.0 & 0.0 \\
\hline 2600 & 0.2 & 0.0 & 0.4 & 0.0 & 0.4 & 0.0 & 0.3 & 0.0 \\
\hline 2605 & 0.0 & 0.0 & 0.0 & 0.0 & 0.0 & 0.0 & 0.0 & 0.0 \\
\hline 2632 & 0.0 & 0.0 & 0.0 & 0.0 & 0.0 & 0.0 & 0.0 & 0.0 \\
\hline 2660 & 0.1 & 0.0 & 0.0 & 0.0 & 0.2 & 0.1 & 0.1 & 0.1 \\
\hline 2672 & 0.0 & 0.0 & 0.0 & 0.0 & 0.0 & 0.0 & 0.0 & 0.0 \\
\hline 2700 & 4.2 & 0.3 & 5.5 & 0.4 & 4.5 & 0.3 & 5.9 & 1.0 \\
\hline 2732 & 0.3 & 0.0 & 0.0 & 0.0 & 0.1 & 0.1 & 0.2 & 0.1 \\
\hline 2750 & 0.0 & 0.0 & 0.0 & 0.0 & 0.0 & 0.0 & 0.0 & 0.0 \\
\hline 2760 & 0.0 & 0.0 & 0.0 & 0.0 & 0.0 & 0.0 & 0.0 & 0.0 \\
\hline 2772 & 0.6 & 0.0 & 0.2 & 0.0 & 1.5 & 0.7 & 0.6 & 0.2 \\
\hline
\end{tabular}




\begin{tabular}{|c|c|c|c|c|c|c|c|c|}
\hline \multirow[b]{2}{*}{$\mathrm{KI}$} & \multicolumn{2}{|c|}{ SgoEstero } & \multicolumn{2}{|c|}{ Mataral } & \multicolumn{2}{|c|}{ Boqueron } & \multicolumn{2}{|c|}{ PteHayes } \\
\hline & media & $\overline{\text { es }}$ & media & es & media & $\overline{\mathrm{es}}$ & media & $\overline{\text { es }}$ \\
\hline 2780 & 0.0 & 0.0 & 0.0 & 0.0 & 0.1 & 0.1 & 0.0 & 0.0 \\
\hline 2800 & 2.4 & 0.2 & 1.7 & 0.1 & 2.9 & 0.2 & 1.9 & 0.1 \\
\hline 2805 & 0.0 & 0.0 & 0.0 & 0.0 & 0.0 & 0.0 & 0.0 & 0.0 \\
\hline 2826 & 0.0 & 0.0 & 0.0 & 0.0 & 0.2 & 0.1 & 0.0 & 0.0 \\
\hline 2850 & 0.1 & 0.0 & 0.0 & 0.0 & 0.0 & 0.0 & 0.0 & 0.0 \\
\hline 2858 & 0.2 & 0.0 & 0.0 & 0.0 & 0.1 & 0.1 & 0.1 & 0.0 \\
\hline 2864 & 0.0 & 0.0 & 0.0 & 0.0 & 0.0 & 0.0 & 0.0 & 0.0 \\
\hline 2900 & 36.1 & 1.2 & 29.5 & 2.2 & 30.6 & 1.4 & 32.3 & 2.2 \\
\hline 2932 & 0.2 & 0.0 & 0.2 & 0.1 & 0.2 & 0.1 & 0.4 & 0.1 \\
\hline 2942 & 0.0 & 0.0 & 0.0 & 0.0 & 0.0 & 0.0 & 0.0 & 0.0 \\
\hline 2951 & 0.1 & 0.0 & 0.3 & 0.1 & 0.0 & 0.0 & 0.2 & 0.1 \\
\hline 2958 & 0.1 & 0.0 & 0.0 & 0.0 & 0.0 & 0.0 & 0.1 & 0.0 \\
\hline 2974 & 1.0 & 0.1 & 0.2 & 0.0 & 0.1 & 0.1 & 0.3 & 0.1 \\
\hline 2981 & 0.0 & 0.0 & 0.0 & 0.0 & 0.0 & 0.0 & 0.0 & 0.0 \\
\hline 2994 & 0.0 & 0.0 & 0.0 & 0.0 & 0.0 & 0.0 & 0.0 & 0.0 \\
\hline 3000 & 1.0 & 0.1 & 1.0 & 0.1 & 1.8 & 0.1 & 1.3 & 0.1 \\
\hline 3030 & 0.0 & 0.0 & 0.0 & 0.0 & 0.1 & 0.1 & 0.1 & 0.0 \\
\hline 3045 & 0.0 & 0.0 & 0.0 & 0.0 & 0.0 & 0.0 & 0.0 & 0.0 \\
\hline 3060 & 0.1 & 0.0 & 0.0 & 0.0 & 0.0 & 0.0 & 0.2 & 0.1 \\
\hline 3074 & 0.2 & 0.0 & 0.0 & 0.0 & 0.0 & 0.0 & 0.1 & 0.0 \\
\hline 3091 & 0.0 & 0.0 & 0.0 & 0.0 & 0.2 & 0.1 & 0.0 & 0.0 \\
\hline 3100 & 11.1 & 0.6 & 9.4 & 0.5 & 10.6 & 1.2 & 13.8 & 1.0 \\
\hline 3130 & 0.1 & 0.0 & 0.1 & 0.0 & 0.1 & 0.0 & 0.4 & 0.1 \\
\hline 3135 & 0.0 & 0.0 & 0.0 & 0.0 & 0.0 & 0.0 & 0.0 & 0.0 \\
\hline 3140 & 0.1 & 0.0 & 0.1 & 0.0 & 0.1 & 0.1 & 0.1 & 0.0 \\
\hline 3150 & 0.1 & 0.0 & 1.0 & 0.2 & 0.1 & 0.0 & 0.4 & 0.1 \\
\hline 3160 & 0.0 & 0.0 & 0.0 & 0.0 & 0.0 & 0.0 & 0.0 & 0.0 \\
\hline 3175 & 4.7 & 0.2 & 1.7 & 0.4 & 0.9 & 0.3 & 2.1 & 0.8 \\
\hline 3187 & 0.0 & 0.0 & 0.0 & 0.0 & 0.0 & 0.0 & 0.1 & 0.0 \\
\hline 3192 & 0.0 & 0.0 & 0.0 & 0.0 & 0.0 & 0.0 & 0.0 & 0.0 \\
\hline 3200 & 0.4 & 0.0 & 0.5 & 0.0 & 0.9 & 0.1 & 0.6 & 0.1 \\
\hline 3208 & 0.0 & 0.0 & 0.0 & 0.0 & 0.0 & 0.0 & 0.0 & 0.0 \\
\hline 3212 & 0.0 & 0.0 & 0.0 & 0.0 & 0.0 & 0.0 & 0.0 & 0.0 \\
\hline 3242 & 0.7 & 0.2 & 0.4 & 0.1 & 2.1 & 1.7 & 0.2 & 0.1 \\
\hline 3257 & 0.3 & 0.0 & 0.4 & 0.1 & 0.1 & 0.0 & 0.2 & 0.0 \\
\hline 3274 & 0.4 & 0.0 & 0.2 & 0.0 & 0.3 & 0.0 & 0.3 & 0.1 \\
\hline 3300 & 4.5 & 0.2 & 3.2 & 0.3 & 5.2 & 0.4 & 4.7 & 0.7 \\
\hline 3330 & 0.0 & 0.0 & 0.4 & 0.1 & 0.0 & 0.0 & 0.1 & 0.0 \\
\hline 3341 & 0.0 & 0.0 & 0.0 & 0.0 & 0.1 & 0.1 & 0.0 & 0.0 \\
\hline 3351 & 0.1 & 0.0 & 1.6 & 0.2 & 0.1 & 0.1 & 0.2 & 0.0 \\
\hline 3357 & 0.0 & 0.0 & 0.8 & 0.1 & 0.0 & 0.0 & 0.0 & 0.0 \\
\hline
\end{tabular}




\begin{tabular}{|c|c|c|c|c|c|c|c|c|}
\hline \multirow[b]{2}{*}{$\mathrm{KI}$} & \multicolumn{2}{|c|}{ SgoEstero } & \multicolumn{2}{|c|}{ Mataral } & \multicolumn{2}{|c|}{ Boqueron } & \multicolumn{2}{|c|}{ PteHayes } \\
\hline & media & $\overline{\text { es }}$ & media & $\overline{\text { es }}$ & media & $\overline{\text { es }}$ & media & es \\
\hline 3372 & 5.9 & 0.3 & 5.1 & 0.5 & 5.0 & 0.3 & 4.0 & 0.4 \\
\hline 3378 & 0.0 & 0.0 & 0.1 & 0.0 & 0.1 & 0.0 & 0.1 & 0.0 \\
\hline 3400 & 0.1 & 0.0 & 0.4 & 0.1 & 0.3 & 0.0 & 0.2 & 0.0 \\
\hline 3404 & 0.1 & 0.0 & 0.1 & 0.0 & 0.1 & 0.1 & 0.1 & 0.0 \\
\hline 3408 & 0.0 & 0.0 & 0.0 & 0.0 & 0.0 & 0.0 & 0.0 & 0.0 \\
\hline 3425 & 0.0 & 0.0 & 0.4 & 0.3 & 0.0 & 0.0 & 0.0 & 0.0 \\
\hline 3445 & 0.4 & 0.1 & 0.1 & 0.0 & 1.1 & 0.7 & 0.2 & 0.1 \\
\hline 3460 & 0.1 & 0.0 & 0.2 & 0.1 & 0.4 & 0.0 & 0.4 & 0.1 \\
\hline 3472 & 0.2 & 0.0 & 0.1 & 0.1 & 0.1 & 0.0 & 0.1 & 0.0 \\
\hline 3480 & 0.0 & 0.0 & 0.1 & 0.0 & 0.0 & 0.0 & 0.1 & 0.1 \\
\hline 3500 & 0.1 & 0.0 & 0.2 & 0.1 & 0.7 & 0.4 & 0.3 & 0.1 \\
\hline 3527 & 0.2 & 0.0 & 0.4 & 0.0 & 0.1 & 0.0 & 0.1 & 0.1 \\
\hline 3548 & 0.0 & 0.0 & 0.5 & 0.1 & 0.1 & 0.1 & 0.2 & 0.1 \\
\hline 3554 & 0.0 & 0.0 & 0.0 & 0.0 & 0.0 & 0.0 & 0.0 & 0.0 \\
\hline 3575 & 1.4 & 0.1 & 1.3 & 0.1 & 1.0 & 0.2 & 0.9 & 0.2 \\
\hline 3625 & 0.0 & 0.0 & 0.1 & 0.0 & 0.0 & 0.0 & 0.0 & 0.0 \\
\hline 3650 & 0.0 & 0.0 & 0.0 & 0.0 & 0.1 & 0.1 & 0.0 & 0.0 \\
\hline 3654 & 0.2 & 0.0 & 0.0 & 0.0 & 0.3 & 0.1 & 0.2 & 0.1 \\
\hline 3670 & 1.2 & 0.1 & 0.4 & 0.1 & 0.7 & 0.0 & 0.6 & 0.1 \\
\hline 3680 & 0.0 & 0.0 & 0.0 & 0.0 & 0.0 & 0.0 & 0.0 & 0.0 \\
\hline 3725 & 0.6 & 0.0 & 2.3 & 0.1 & 0.5 & 0.0 & 0.5 & 0.1 \\
\hline 3750 & 0.2 & 0.0 & 0.3 & 0.1 & 0.4 & 0.1 & 0.5 & 0.2 \\
\hline 3766 & 3.4 & 0.2 & 1.3 & 0.2 & 2.1 & 0.2 & 1.8 & 0.3 \\
\hline 3773 & 1.1 & 0.1 & 1.2 & 0.3 & 1.3 & 0.2 & 1.5 & 0.3 \\
\hline 3800 & 0.9 & 0.0 & 0.9 & 0.1 & 1.9 & 0.1 & 1.7 & 0.2 \\
\hline 3823 & 0.2 & 0.0 & 0.8 & 0.1 & 0.7 & 0.0 & 0.6 & 0.1 \\
\hline 3830 & 0.0 & 0.0 & 0.0 & 0.0 & 0.0 & 0.0 & 0.0 & 0.0 \\
\hline 3844 & 0.2 & 0.0 & 0.3 & 0.1 & 0.3 & 0.0 & 0.3 & 0.1 \\
\hline 3857 & 0.0 & 0.0 & 0.0 & 0.0 & 0.0 & 0.0 & 0.0 & 0.0 \\
\hline 3866 & 3.0 & 0.1 & 2.3 & 0.3 & 4.7 & 0.6 & 3.3 & 0.5 \\
\hline 3877 & 0.0 & 0.0 & 0.1 & 0.0 & 0.3 & 0.1 & 0.3 & 0.1 \\
\hline 3920 & 1.2 & 0.1 & 3.2 & 0.3 & 1.2 & 0.2 & 1.1 & 0.2 \\
\hline 3941 & 1.0 & 0.1 & 3.0 & 0.3 & 1.3 & 0.1 & 1.8 & 0.3 \\
\hline 3965 & 4.7 & 0.3 & 8.9 & 1.1 & 6.0 & 0.7 & 6.3 & 1.1 \\
\hline 3971 & 0.8 & 0.1 & 1.4 & 0.2 & 1.3 & 0.2 & 1.4 & 0.2 \\
\hline 4003 & 1.1 & 0.1 & 1.7 & 0.2 & 1.5 & 0.1 & 1.8 & 0.3 \\
\hline 4046 & 0.4 & 0.1 & 0.7 & 0.1 & 0.5 & 0.1 & 0.5 & 0.1 \\
\hline 4125 & 0.3 & 0.0 & 0.5 & 0.1 & 0.3 & 0.1 & 0.4 & 0.1 \\
\hline
\end{tabular}


Tabla 6c. Composición de los HC cuticulares de T. garciabesi y T. patagonica.

\begin{tabular}{|c|c|c|c|c|c|c|}
\hline \multirow[b]{3}{*}{ KI } & \multicolumn{4}{|c|}{ T. garciabesi } & \multirow{2}{*}{\multicolumn{2}{|c|}{$\frac{\text { T. patagonica }}{\text { SantaFe }}$}} \\
\hline & \multicolumn{2}{|c|}{ LaRioja } & \multicolumn{2}{|c|}{ SgoEstero } & & \\
\hline & media & $\overline{\mathrm{es}}$ & media & $\overline{\text { es }}$ & media & es \\
\hline 2100 & 0.0 & 0.0 & 0.0 & 0.0 & 0.2 & 0.0 \\
\hline 2200 & 0.1 & 0.0 & 0.1 & 0.0 & 0.4 & 0.0 \\
\hline 2300 & 0.4 & 0.1 & 0.2 & 0.0 & 0.2 & 0.0 \\
\hline 2330 & 0.0 & 0.0 & 0.0 & 0.0 & 0.0 & 0.0 \\
\hline 2392 & 0.0 & 0.0 & 0.0 & 0.0 & 0.0 & 0.0 \\
\hline 2400 & 0.4 & 0.0 & 0.2 & 0.0 & 1.1 & 0.1 \\
\hline 2431 & 0.0 & 0.0 & 0.0 & 0.0 & 0.0 & 0.0 \\
\hline 2460 & 0.0 & 0.0 & 0.1 & 0.0 & 0.0 & 0.0 \\
\hline 2500 & 2.4 & 0.2 & 2.6 & 0.2 & 0.3 & 0.0 \\
\hline 2535 & 0.0 & 0.0 & 0.1 & 0.0 & 0.6 & 0.1 \\
\hline 2574 & 0.0 & 0.0 & 0.0 & 0.0 & 0.0 & 0.0 \\
\hline 2600 & 1.9 & 0.1 & 1.5 & 0.2 & 0.2 & 0.0 \\
\hline 2605 & 0.0 & 0.0 & 0.0 & 0.0 & 0.0 & 0.0 \\
\hline 2632 & 0.1 & 0.0 & 0.1 & 0.0 & 0.0 & 0.0 \\
\hline 2660 & 0.6 & 0.1 & 0.8 & 0.1 & 0.1 & 0.0 \\
\hline 2672 & 0.0 & 0.0 & 0.0 & 0.0 & 0.0 & 0.0 \\
\hline 2700 & 16.4 & 1.3 & 14.5 & 1.2 & 3.4 & 0.2 \\
\hline 2732 & 0.4 & 0.0 & 0.6 & 0.0 & 0.1 & 0.0 \\
\hline 2750 & 0.0 & 0.0 & 0.0 & 0.0 & 0.0 & 0.0 \\
\hline 2760 & 0.0 & 0.0 & 0.0 & 0.0 & 0.0 & 0.0 \\
\hline 2772 & 4.4 & 0.5 & 4.2 & 0.3 & 0.4 & 0.0 \\
\hline 2780 & 0.1 & 0.0 & 0.1 & 0.0 & 0.0 & 0.0 \\
\hline 2800 & 4.6 & 0.3 & 3.9 & 0.3 & 2.1 & 0.1 \\
\hline 2805 & 0.0 & 0.0 & 0.0 & 0.0 & 0.0 & 0.0 \\
\hline 2826 & 0.0 & 0.0 & 0.0 & 0.0 & 0.8 & 0.2 \\
\hline 2850 & 0.3 & 0.1 & 0.0 & 0.0 & 0.0 & 0.0 \\
\hline 2858 & 1.0 & 0.1 & 0.6 & 0.0 & 0.1 & 0.0 \\
\hline 2864 & 0.0 & 0.0 & 0.0 & 0.0 & 0.0 & 0.0 \\
\hline 2900 & 21.1 & 1.1 & 21.9 & 0.9 & 43.1 & 1.6 \\
\hline 2932 & 0.1 & 0.0 & 0.3 & 0.0 & 0.3 & 0.0 \\
\hline 2942 & 0.0 & 0.0 & 0.1 & 0.0 & 0.0 & 0.0 \\
\hline 2951 & 0.5 & 0.0 & 0.6 & 0.0 & 0.0 & 0.0 \\
\hline 2958 & 0.1 & 0.0 & 0.3 & 0.0 & 0.0 & 0.0 \\
\hline 2974 & 2.1 & 0.1 & 1.2 & 0.1 & 0.3 & 0.0 \\
\hline 2981 & 0.1 & 0.0 & 0.2 & 0.0 & 0.0 & 0.0 \\
\hline 2994 & 0.9 & 0.1 & 0.7 & 0.1 & 0.0 & 0.0 \\
\hline 3000 & 0.5 & 0.0 & 0.7 & 0.0 & 0.8 & 0.0 \\
\hline 3030 & 0.8 & 0.2 & 0.4 & 0.2 & 0.9 & 0.2 \\
\hline
\end{tabular}




\begin{tabular}{|c|c|c|c|c|c|c|}
\hline \multirow[b]{3}{*}{$\mathrm{KI}$} & \multicolumn{4}{|c|}{ T. garciabesi } & \multirow{2}{*}{\multicolumn{2}{|c|}{$\frac{\text { T. patagonica }}{\text { SantaFe }}$}} \\
\hline & \multicolumn{2}{|c|}{ LaRioja } & \multicolumn{2}{|c|}{ SgoEstero } & & \\
\hline & media & es & media & es & media & es \\
\hline 3045 & 0.0 & 0.0 & 0.0 & 0.0 & 0.0 & 0.0 \\
\hline 3060 & 0.2 & 0.0 & 0.1 & 0.0 & 0.0 & 0.0 \\
\hline 3074 & 0.3 & 0.0 & 0.2 & 0.0 & 0.0 & 0.0 \\
\hline 3091 & 0.4 & 0.1 & 0.2 & 0.0 & 0.0 & 0.0 \\
\hline 3100 & 3.3 & 0.4 & 4.9 & 0.4 & 6.6 & 0.2 \\
\hline 3130 & 0.0 & 0.0 & 0.1 & 0.0 & 0.2 & 0.1 \\
\hline 3135 & 0.0 & 0.0 & 0.0 & 0.0 & 0.0 & 0.0 \\
\hline 3140 & 0.0 & 0.0 & 0.1 & 0.0 & 0.1 & 0.0 \\
\hline 3150 & 0.0 & 0.0 & 0.2 & 0.0 & 0.0 & 0.0 \\
\hline 3160 & 0.0 & 0.0 & 0.0 & 0.0 & 0.0 & 0.0 \\
\hline 3175 & 5.7 & 0.6 & 3.7 & 0.4 & 3.0 & 0.2 \\
\hline 3187 & 0.2 & 0.0 & 0.4 & 0.0 & 0.0 & 0.0 \\
\hline 3192 & 0.0 & 0.0 & 0.0 & 0.0 & 0.0 & 0.0 \\
\hline 3200 & 0.1 & 0.0 & 0.2 & 0.0 & 0.3 & 0.0 \\
\hline 3208 & 0.3 & 0.0 & 0.1 & 0.0 & 0.0 & 0.0 \\
\hline 3212 & 0.0 & 0.0 & 0.0 & 0.0 & 0.0 & 0.0 \\
\hline 3242 & 0.8 & 0.1 & 0.5 & 0.1 & 4.5 & 1.2 \\
\hline 3257 & 0.4 & 0.0 & 0.2 & 0.0 & 0.2 & 0.0 \\
\hline 3274 & 0.1 & 0.0 & 0.1 & 0.0 & 0.4 & 0.0 \\
\hline 3300 & 1.7 & 0.2 & 2.9 & 0.2 & 3.0 & 0.2 \\
\hline 3330 & 0.0 & 0.0 & 0.0 & 0.0 & 0.0 & 0.0 \\
\hline 3341 & 0.0 & 0.0 & 0.0 & 0.0 & 0.0 & 0.0 \\
\hline 3351 & 0.0 & 0.0 & 0.1 & 0.0 & 0.0 & 0.0 \\
\hline 3357 & 0.0 & 0.0 & 0.2 & 0.0 & 0.0 & 0.0 \\
\hline 3372 & 1.3 & 0.1 & 0.9 & 0.0 & 7.3 & 0.4 \\
\hline 3378 & 0.1 & 0.0 & 0.0 & 0.0 & 0.0 & 0.0 \\
\hline 3400 & 0.3 & 0.0 & 0.1 & 0.0 & 0.0 & 0.0 \\
\hline 3404 & 0.2 & 0.0 & 0.1 & 0.0 & 0.0 & 0.0 \\
\hline 3408 & 0.0 & 0.0 & 0.0 & 0.0 & 0.0 & 0.0 \\
\hline 3425 & 0.0 & 0.0 & 0.0 & 0.0 & 0.0 & 0.0 \\
\hline 3445 & 1.4 & 0.2 & 0.8 & 0.3 & 0.8 & 0.2 \\
\hline 3460 & 0.1 & 0.0 & 0.2 & 0.0 & 0.1 & 0.0 \\
\hline 3472 & 0.2 & 0.0 & 0.1 & 0.0 & 0.0 & 0.0 \\
\hline 3480 & 0.0 & 0.0 & 0.0 & 0.0 & 0.0 & 0.0 \\
\hline 3500 & 0.2 & 0.1 & 0.2 & 0.0 & 0.0 & 0.0 \\
\hline 3527 & 0.4 & 0.0 & 0.2 & 0.0 & 0.0 & 0.0 \\
\hline 3548 & 0.0 & 0.0 & 0.1 & 0.0 & 0.0 & 0.0 \\
\hline 3554 & 0.1 & 0.0 & 0.4 & 0.0 & 0.0 & 0.0 \\
\hline 3575 & 1.1 & 0.1 & 1.0 & 0.1 & 0.9 & 0.1 \\
\hline 3625 & 0.2 & 0.0 & 0.1 & 0.0 & 0.0 & 0.0 \\
\hline
\end{tabular}




\begin{tabular}{|c|c|c|c|c|c|c|}
\hline \multirow[b]{3}{*}{ KI } & \multicolumn{4}{|c|}{ T. garciabesi } & \multirow{2}{*}{\multicolumn{2}{|c|}{$\begin{array}{c}\text { T. patagonica } \\
\text { SantaFe }\end{array}$}} \\
\hline & \multicolumn{2}{|c|}{ LaRioja } & \multicolumn{2}{|c|}{ SgoEstero } & & \\
\hline & media & es & media & es & media & es \\
\hline 3650 & 0.6 & 0.1 & 0.4 & 0.2 & 0.0 & 0.0 \\
\hline 3654 & 0.1 & 0.0 & 0.1 & 0.1 & 0.0 & 0.0 \\
\hline 3670 & 1.1 & 0.1 & 1.2 & 0.1 & 0.7 & 0.0 \\
\hline 3680 & 0.0 & 0.0 & 0.0 & 0.0 & 0.0 & 0.0 \\
\hline 3725 & 1.5 & 0.1 & 1.4 & 0.2 & 0.6 & 0.0 \\
\hline 3750 & 0.7 & 0.1 & 1.2 & 0.1 & 0.0 & 0.0 \\
\hline 3766 & 0.1 & 0.0 & 0.7 & 0.1 & 1.3 & 0.1 \\
\hline 3773 & 4.6 & 0.4 & 4.9 & 0.2 & 0.9 & 0.1 \\
\hline 3800 & 1.7 & 0.1 & 1.7 & 0.1 & 1.5 & 0.1 \\
\hline 3823 & 0.5 & 0.0 & 0.4 & 0.0 & 0.3 & 0.0 \\
\hline 3830 & 0.5 & 0.1 & 0.3 & 0.1 & 0.0 & 0.0 \\
\hline 3844 & 0.2 & 0.0 & 0.4 & 0.0 & 0.2 & 0.0 \\
\hline 3857 & 0.0 & 0.0 & 0.0 & 0.0 & 0.0 & 0.0 \\
\hline 3866 & 2.8 & 0.2 & 2.7 & 0.2 & 2.6 & 0.2 \\
\hline 3877 & 0.4 & 0.0 & 0.4 & 0.0 & 0.1 & 0.0 \\
\hline 3920 & 1.2 & 0.1 & 1.2 & 0.1 & 1.2 & 0.1 \\
\hline 3941 & 0.6 & 0.1 & 1.9 & 0.2 & 0.5 & 0.1 \\
\hline 3965 & 4.1 & 0.3 & 5.3 & 0.4 & 4.0 & 0.4 \\
\hline 3971 & 0.0 & 0.0 & 0.0 & 0.0 & 0.4 & 0.0 \\
\hline 4003 & 1.2 & 0.1 & 1.3 & 0.1 & 1.1 & 0.1 \\
\hline 4046 & 0.1 & 0.0 & 0.3 & 0.0 & 0.7 & 0.1 \\
\hline 4125 & 0.0 & 0.0 & 0.2 & 0.0 & 0.0 & 0.0 \\
\hline
\end{tabular}

Figura 20. Perfiles de diferentes poblaciones de T. sordida analizados por CGC. Tiempo de elución en la abscisa, intensidad de la señal en la ordenada.

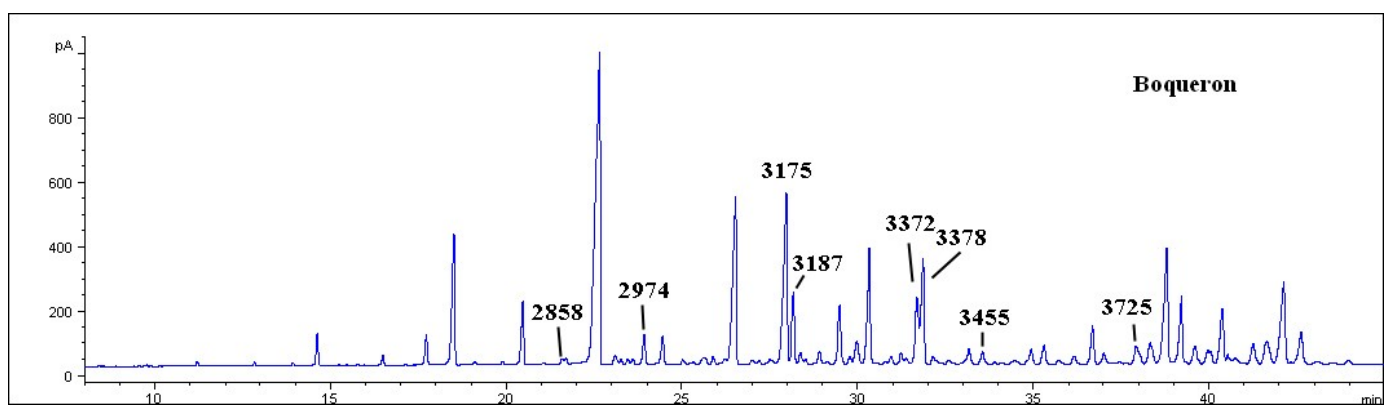

Los números corresponden al KI de los hidrocarburos empleados en el DA

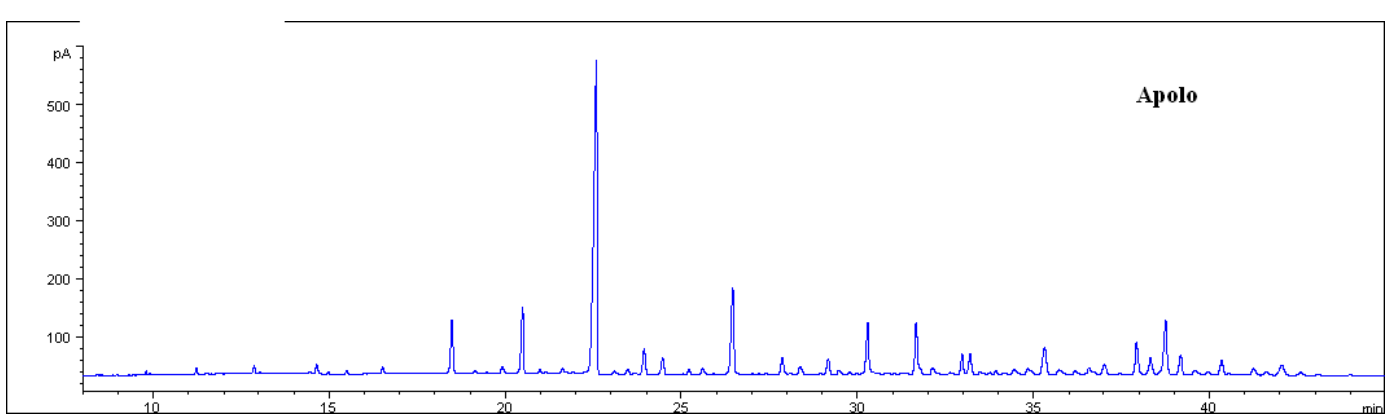



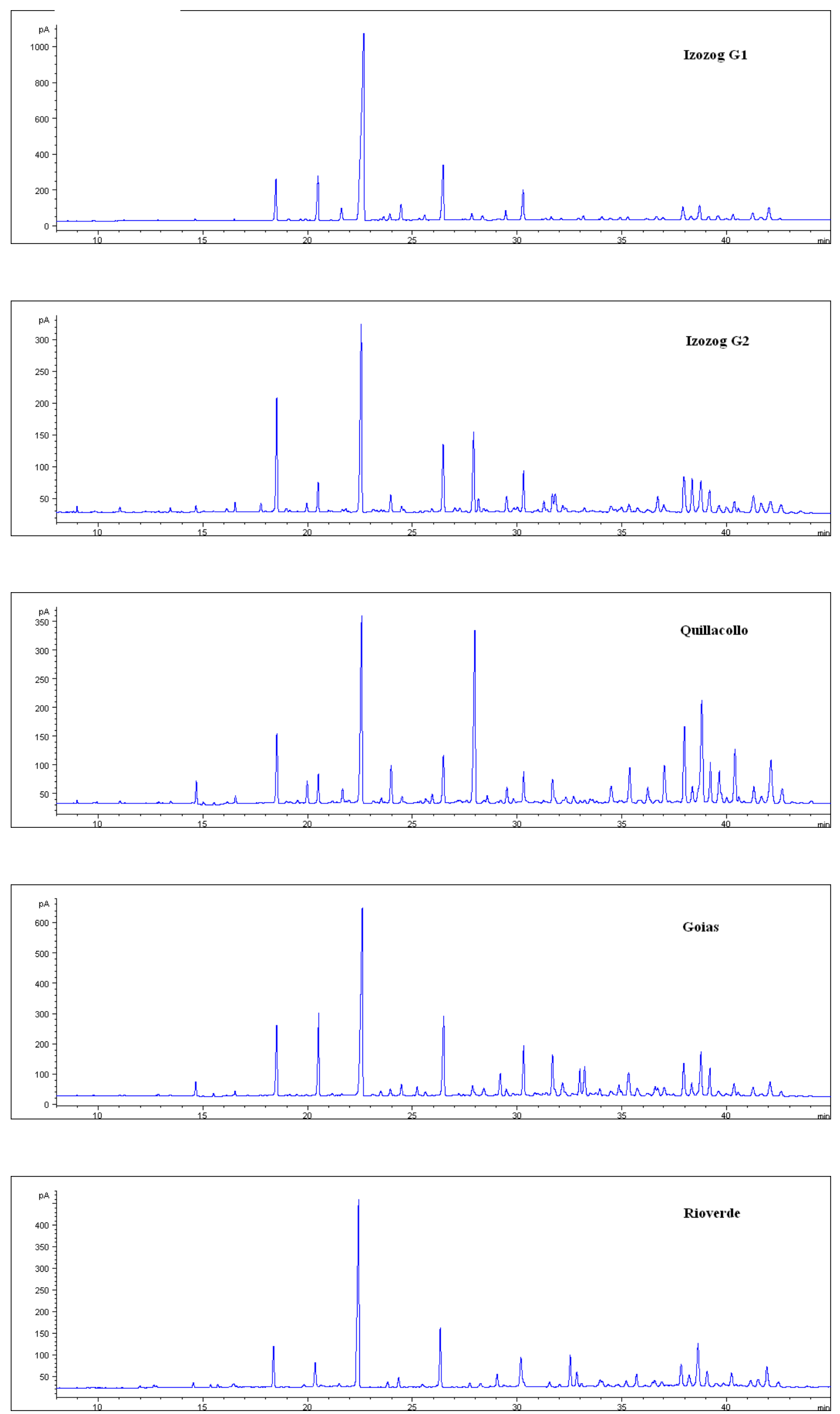

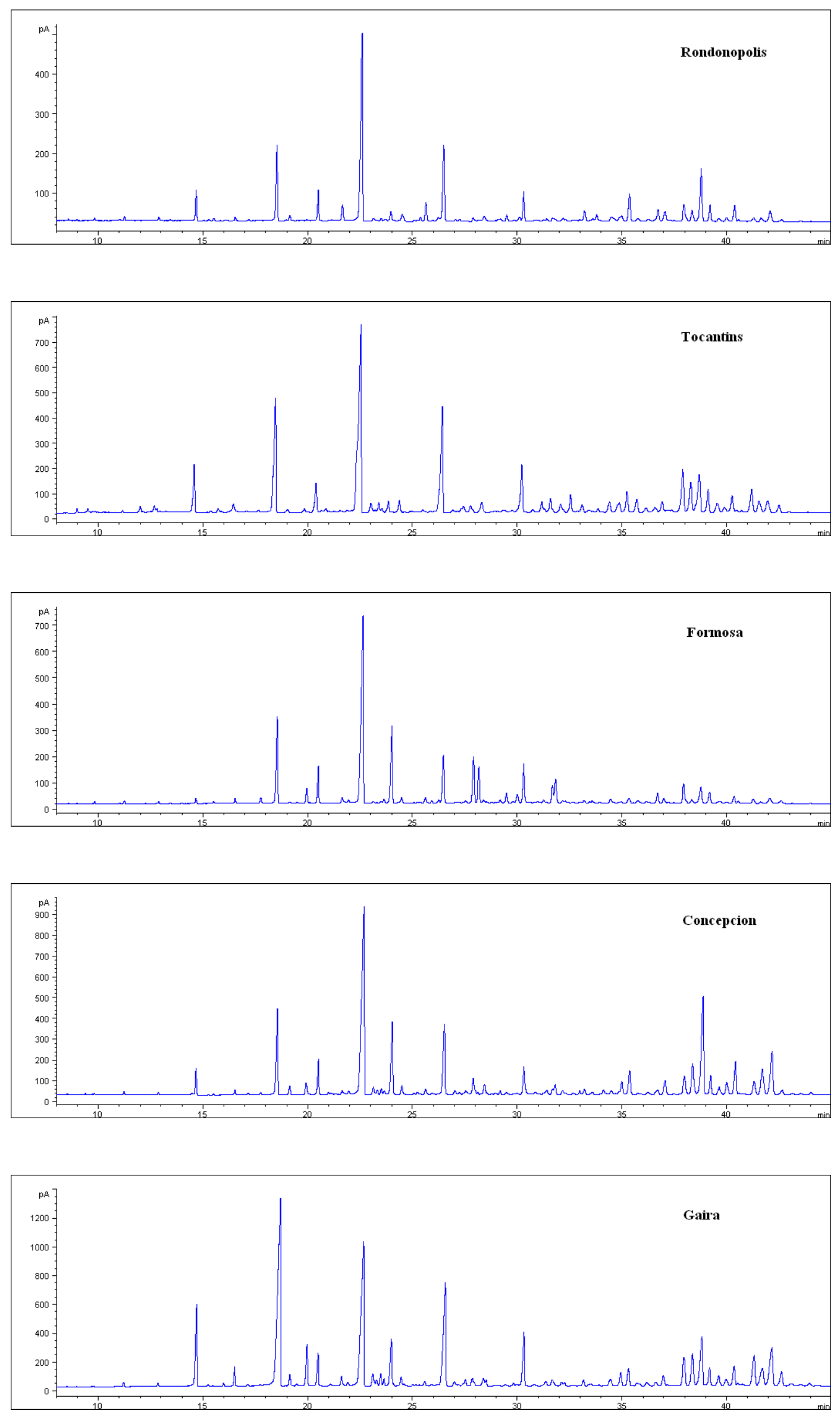

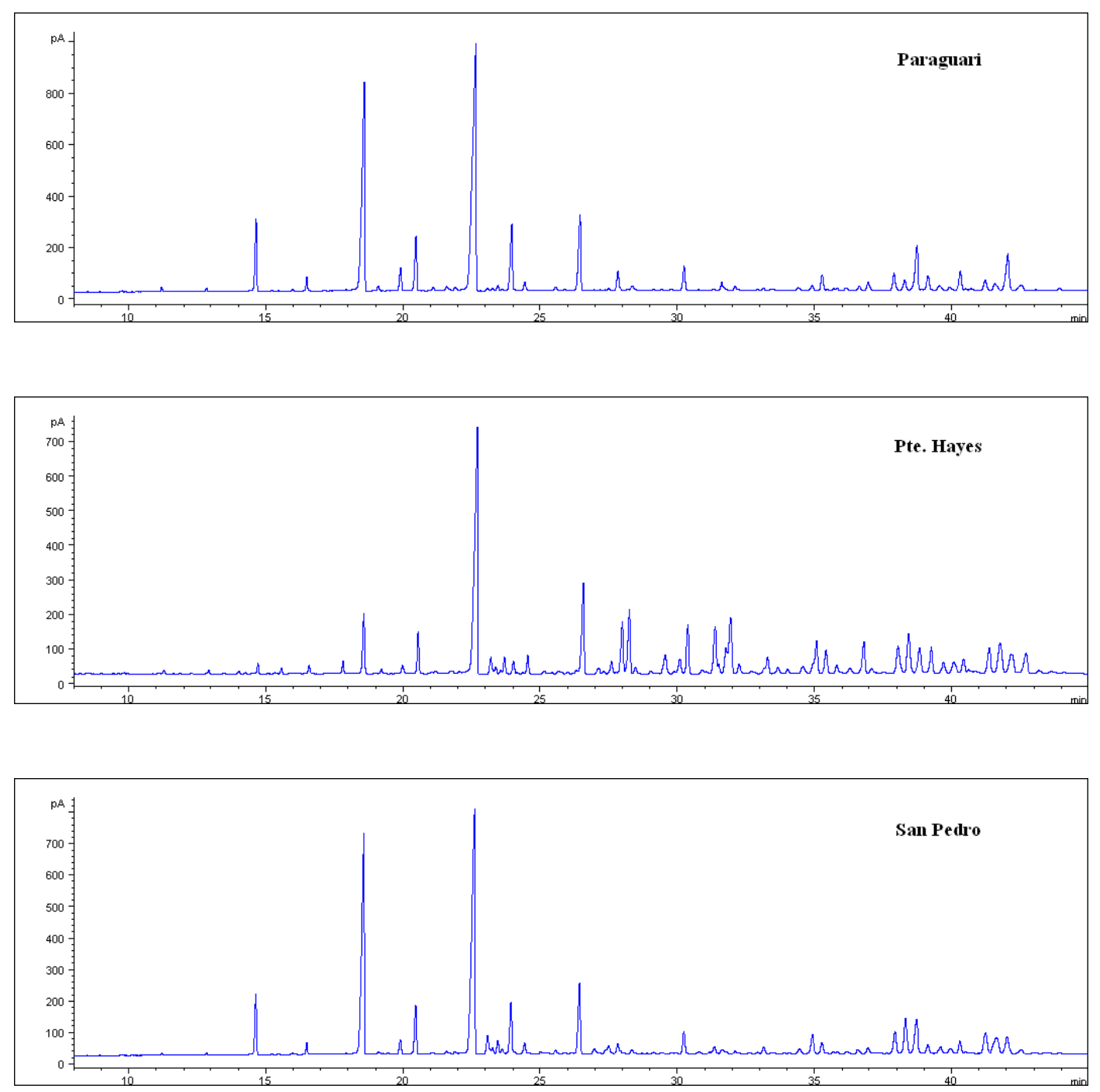

Figura 21. Perfiles de diferentes poblaciones de T. garciabesi analizados por CGC.
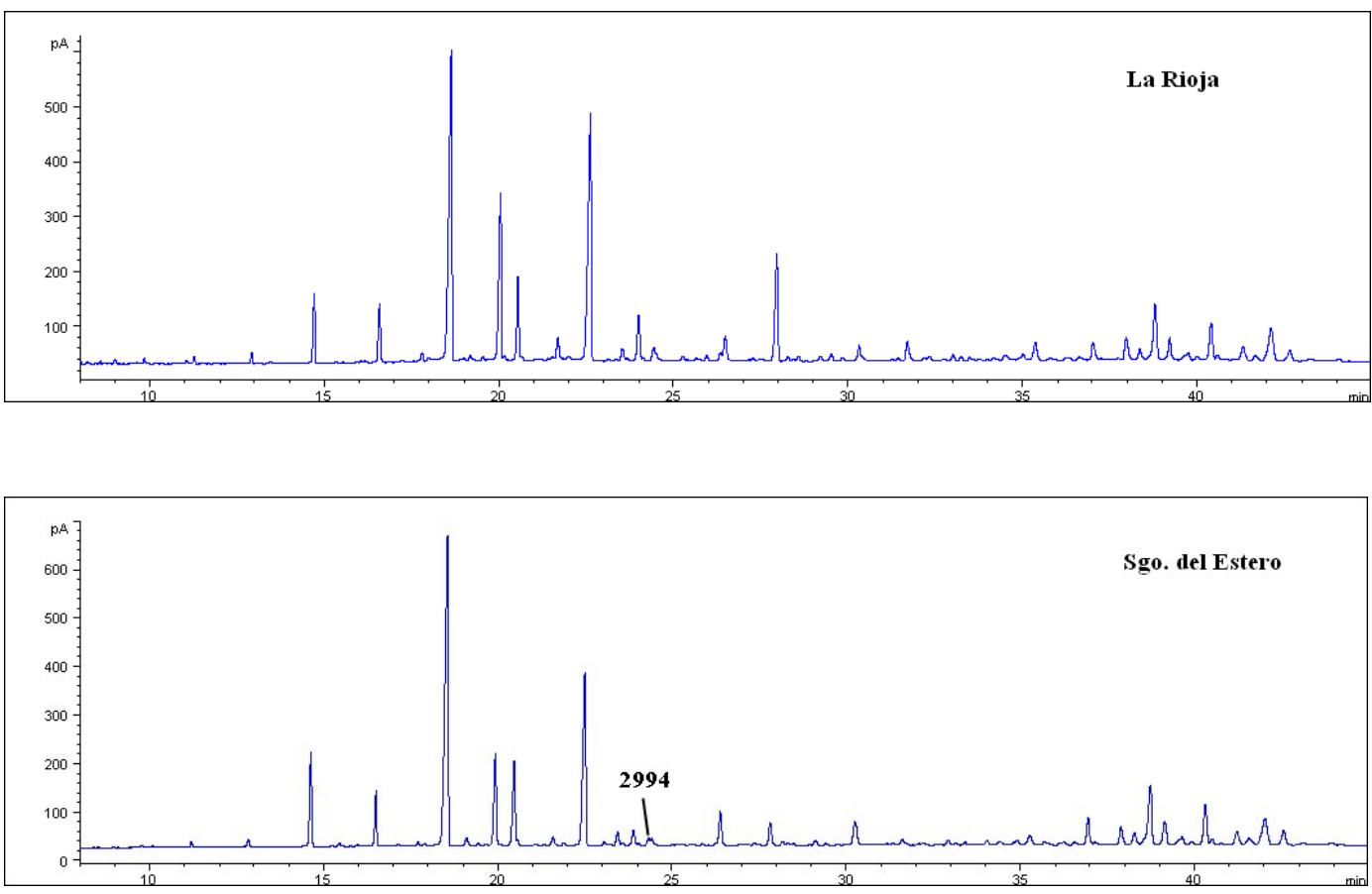
Figura 22. Perfiles de diferentes poblaciones de $T$. guasayana analizados por CGC.
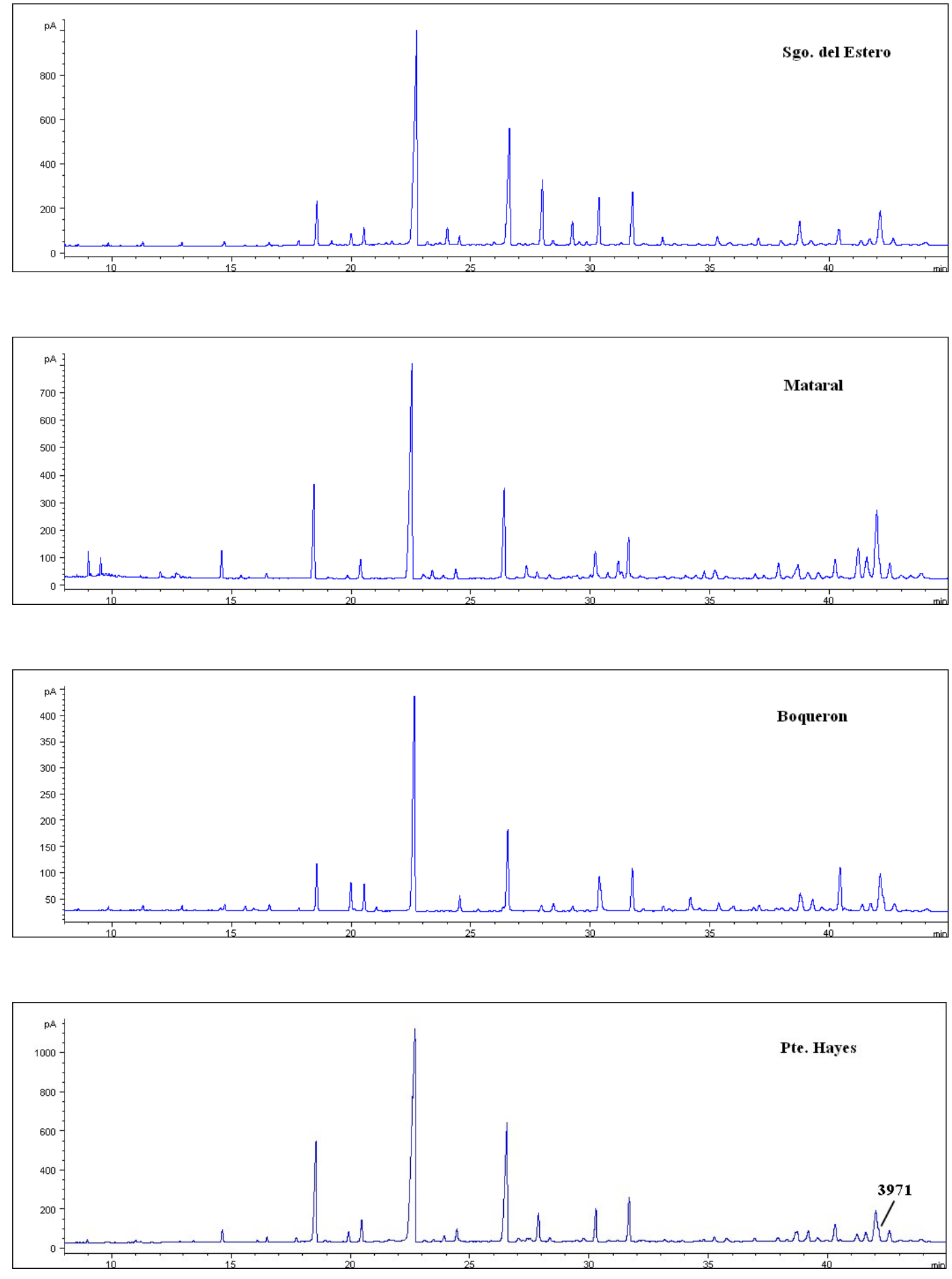

Figura 23. Perfil de CGC de los ejemplares cruza entre T. guasayana y T. sordida (Tg x Ts).

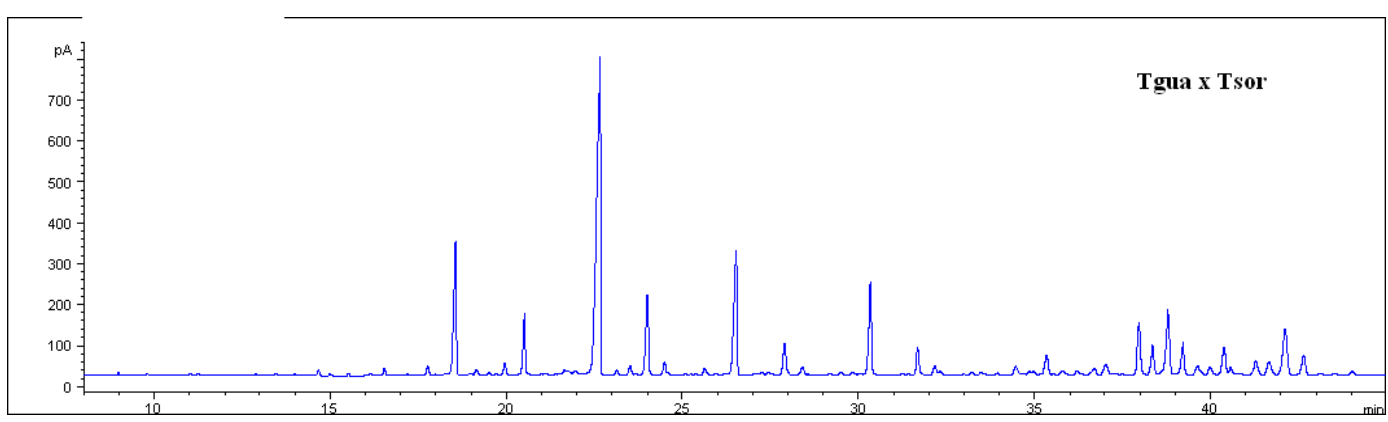


Figura 24. Perfil de CGC de T. patagonica.

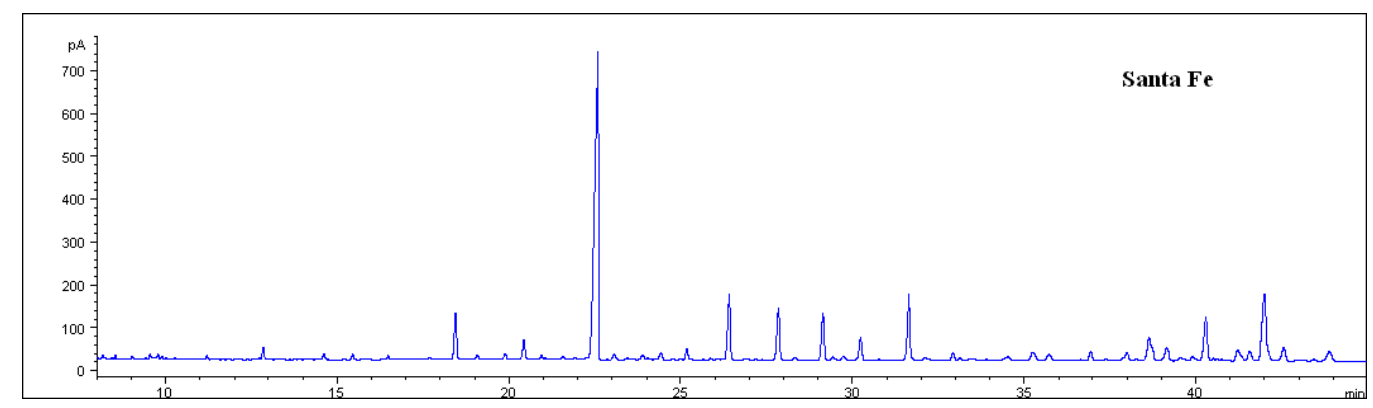

Los números corresponden al KI de los hidrocarburos empleados en el DA

Tras la observación de los distintos perfiles de $\mathrm{HC}$ se puede generalizar que en todas las especies predomina el $n$-C29 sobre los otros picos. Los patrones más complejos son exhibidos por T. garciabesi y $T$. sordida, en esta última especialmente por las poblaciones de Quillacollo e Izozog G2 (Bolivia) y la región occidental de Paraguay.

T. garciabesi muestra de manera característica una alta proporción del 3-metil C27 y 3-metil C31 (KIs 2772 y 3175 respectivamente), la presencia de los HCs 7,13,17-trimetil C29 a C31 (KIs 2994, 3091 y 3192) no detectados en las otras especies y una proporción variable del n-C27 que depende del sexo del ejemplar (mayor proporción en las hembras que en los machos). Los ejemplares de T. sordida de Quillacollo presentan una alta proporción del 3metil C31 (KI 3175) así como también de hidrocarburos ramificados de cadena larga. Las poblaciones de Izozog G2 y la región occidental de Paraguay se caracterizan por la abundancia de los HC 3-metil C31, 11,15,21-trimetil C31, 3-metil C33 y 5,17- y 5,19-dimetil C33 junto al 11,17,21-trimetil C33 (KIs 3175, 3187, 3372 y 3378).

En cambio las distintas poblaciones analizadas de T. guasayana y la de T. patagonica muestran un perfil simple, caracterizado por la ausencia de los numerosos $\mathrm{HC}$ ramificados encontrados en las otras especies, y por la mayor abundancia relativa de trimetil isómeros 13,x,X- del C37 con KI 3766 y dimetil isómeros 5,x- del C39 con KI 3971.

Los ejemplares designados como "Tg x Ts", cruzamientos entre T. guasayana y $T$. sordida, presentan un perfil mixto entre estas especies, por un lado con escasos picos, pero con una gran abundancia del 3-metil C29 (KI 2974) y la ausencia de los HC característicos de T. guasayana (KIs 3766 y 3971).

\section{1. 3. Análisis taxonómico basado en el patrón de hidrocarburos cuticulares}

Aquí se presentan los resultados obtenidos tanto en los análisis preliminares mediante PCA, como en los análisis mediante DA. 
$\underline{\text { PCA de las distintas especies del subcomplejo T. sordida }}$

El PCA realizado en las distintas especies que integran el subcomplejo muestra que presentan una visible heterogeneidad, con excepción de $T$. garciabesi y $T$. patagonica. En el caso de $T$. patagonica no se hizo este análisis para la especie en particular ya que sólo se cuenta con muestras de una localidad (tabla 3 ).

Los ejemplares de T.sordida de Brasil resultaron homogéneos en relación a su distribución geográfica (figura 25), en cambio los de Bolivia y Paraguay se segregaron en dos grupos principales que comprenden las distintas localidades estudiadas (figuras 26 y 27).

Figura 25. Gráfico de puntajes canónicos de los ejemplares de T. sordida de Brasil

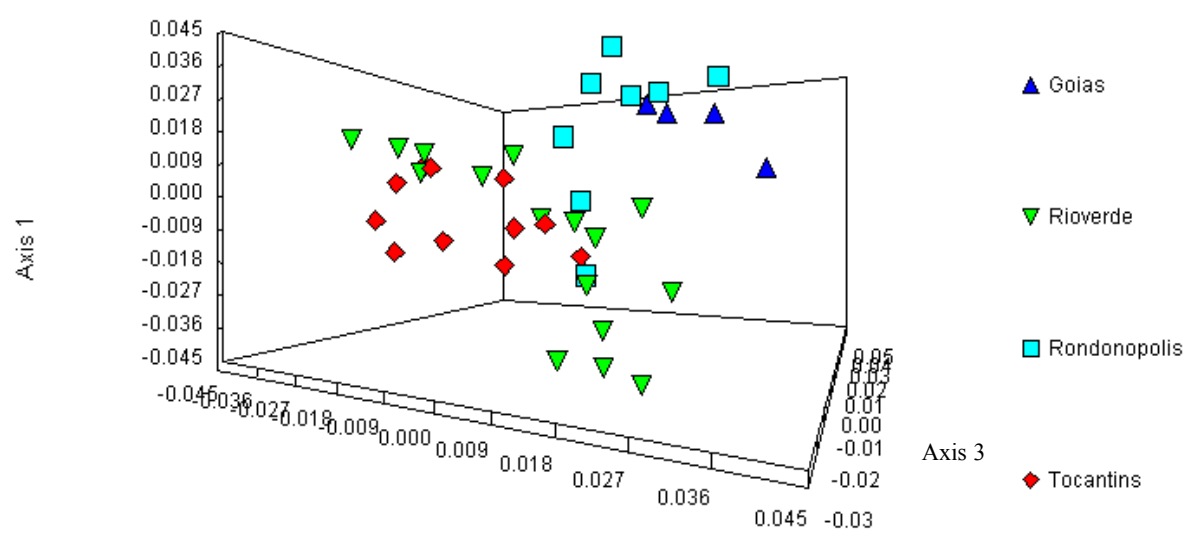

Axis 2

HC con mayor contribución relativa (KIs): 2500, 2700, 2900, 2932, 3242, 3300, 3378, 3425, 3445, 3508, 3600, $3607,3654,3725$

Figura 26. Gráfico de puntajes canónicos de los ejemplares de T. sordida de Bolivia.

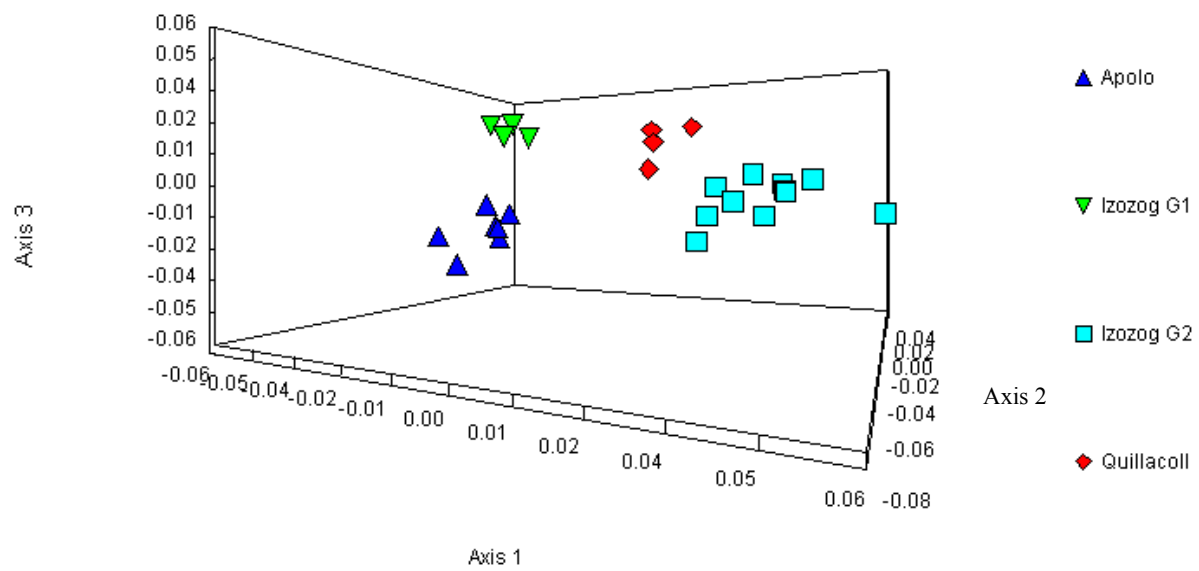

HC con mayor contribución relativa (KIs): 2400, 2500, 2700, 2800, 2858, 2900, 3058, 3175, 3257, 3274, 3300, $3372,3378,3400,3445,3455,3654$ 
Figura 27. Gráfico de puntajes canónicos de los ejemplares de T. sordida de Paraguay

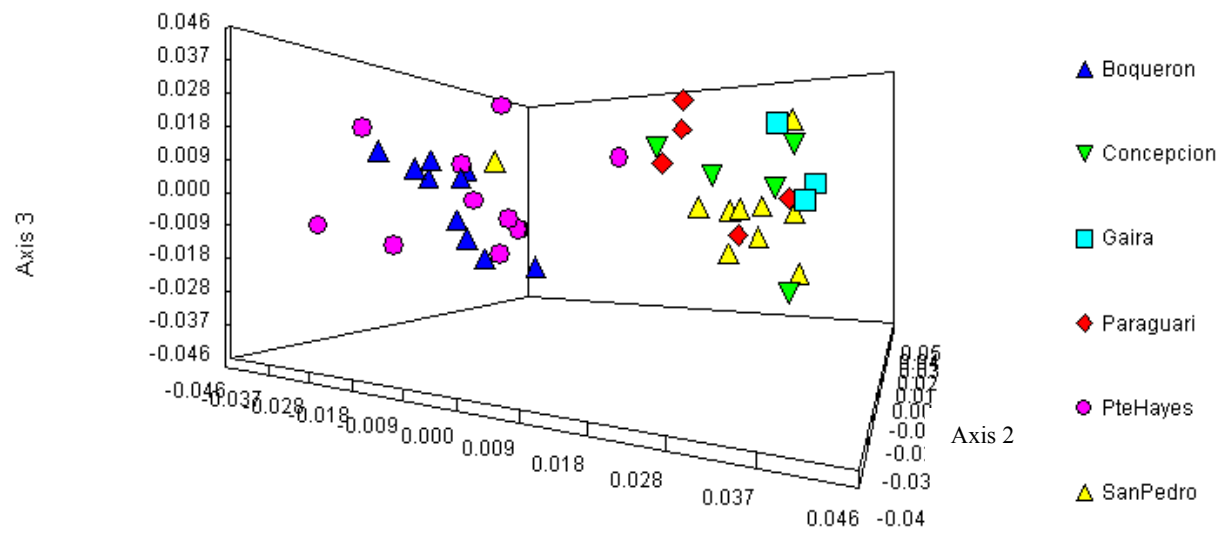

Axis 1

HC con mayor contribución relativa (KIs): 2500, 2700, 2732, 2772, 2826, 2900, 2974, 3100, 3175, 3187, 3300, $3378,3445,3654,3773,3965$

No se hizo este análisis para el caso de Argentina en particular dado que las muestras provenían de una sola región (tabla 3), sin embargo estos ejemplares se ubicaron en uno de los grandes grupos al analizarlos junto con los de otro país (figura 28).

Figura 28. Gráfico de puntajes canónicos de los ejemplares de $T$. sordida de Argentina, Bolivia y Paraguay.

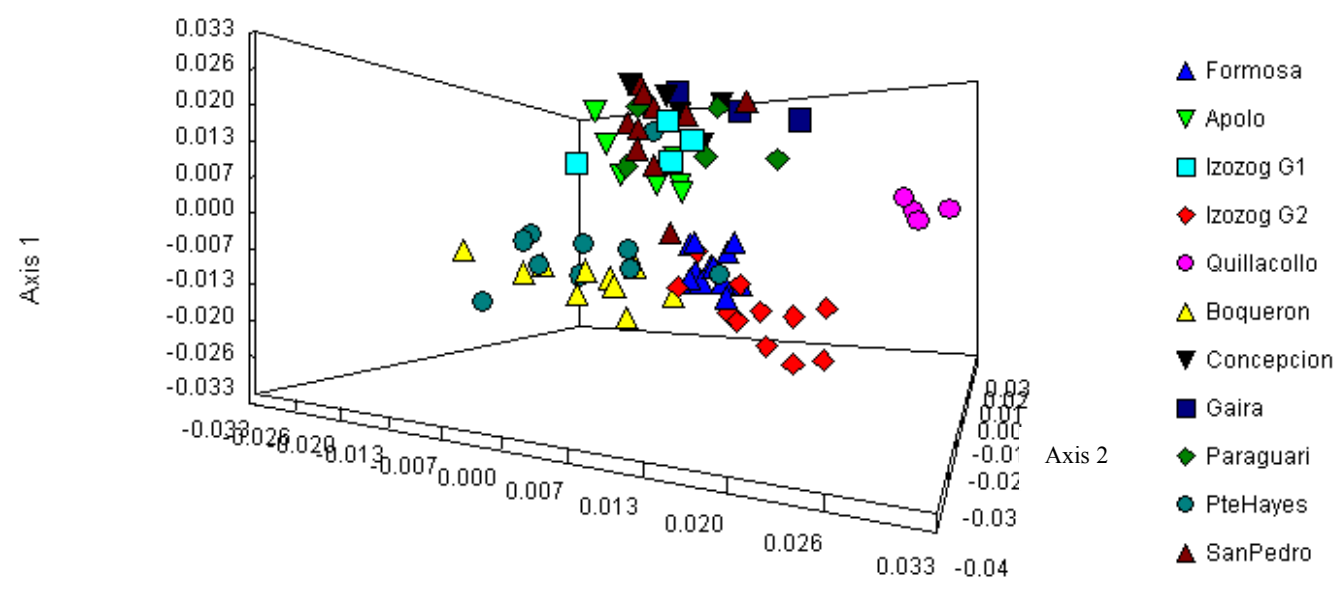

Axis 3

HC con mayor contribución relativa (KIs): 2400, 2500, 2700, 2772, 2800, 2858, 2900, 2974, 3100, 3175, 3187, $3242,3300,3351,3372,3378,3607,3654,3748$

Finalmente, al incluir todas las localidades en el análisis, nuevamente se observa esa segregación de los especímenes en dos grupos principales (figura 29). 
Figura 29. Gráfico de puntajes canónicos de los ejemplares de T. sordida de Argentina, Bolivia, Brasil y Paraguay.

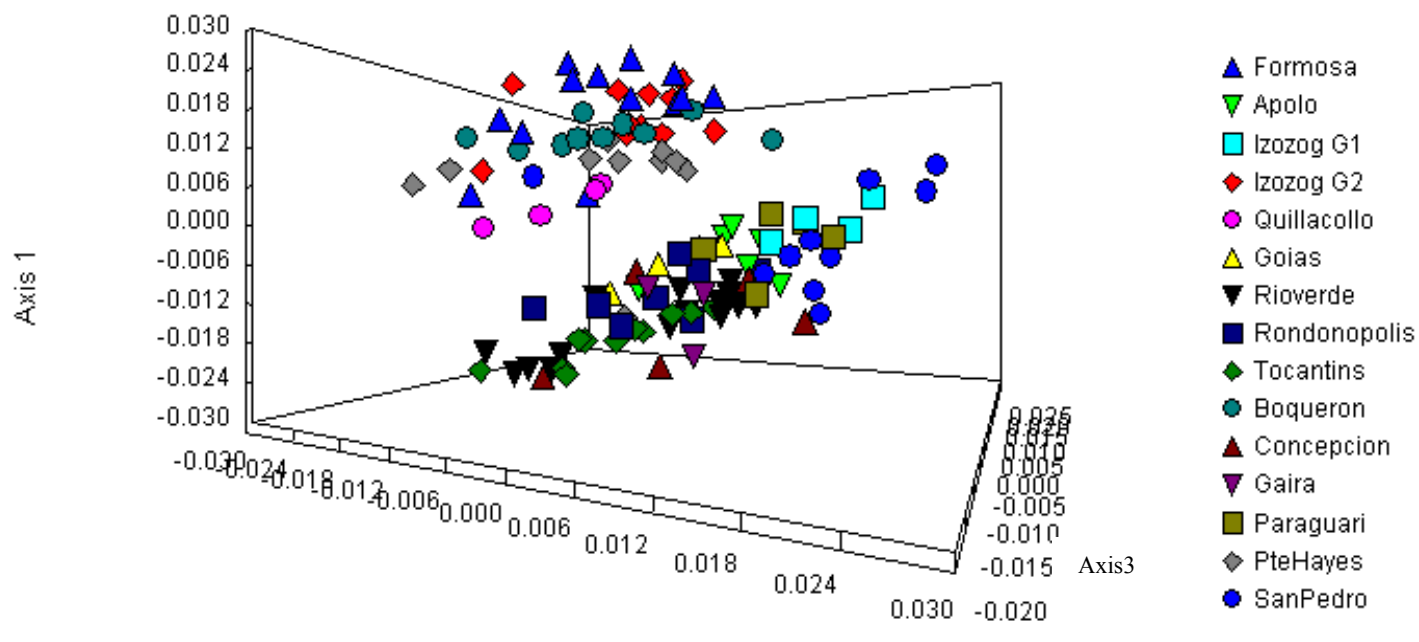

Axis 2

HC con mayor contribución relativa (KIs): 2400, 2500, 2700, 2772, 2900, 2974, 3100, 3175, 3187, 3242, 3300, $3351,3378,3607,3654$

Estos dos grupos principales, llamados de ahora en adelante "grupo 1" y "grupo 2" se componen de las siguientes poblaciones:

- el grupo 1 comprende a los insectos de Brasil, región oriental de Paraguay (Concepción, Gaira, Paraguari y San Pedro) y Bolivia (Apolo e Izozog G1)

- el grupo 2 comprende a los insectos de Argentina (Formosa), región occidental de Paraguay (Boquerón y Presidente Hayes) y Bolivia (Izozog G2).

Figura 30. Dendrograma de las poblaciones de $T$. sordida derivado a partir de los puntajes canónicos del PCA

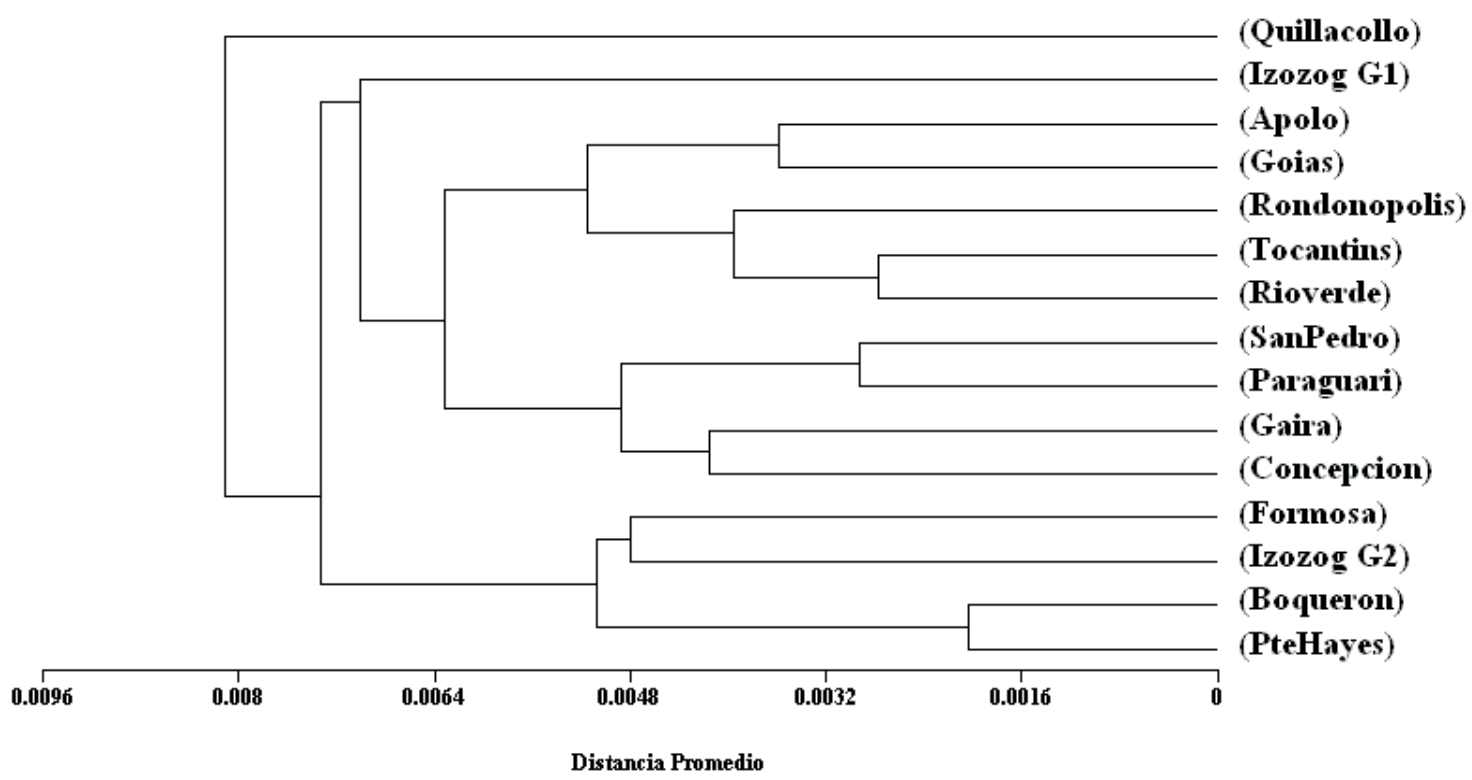


Los ejemplares de Quillacollo parecen presentar la mayor disimilitud respecto a sus conespecíficos. El resto de los insectos dentro de cada grupo, se distribuyen aproximadamente de acuerdo a su proximidad geográfica, excepto los ejemplares de Izozog que quedan divididos entre los dos grupos principales y los ejemplares de la región andina de Bolivia (Apolo) que muestran una gran similitud con los del este de Brasil (Goias) agrupándose junto a éstos (figura 30).

Como ya se mencionó, los especimenes de $T$. garciabesi no parecen presentar una alta diferenciación en relación a la distribución geográfica, mostrado solo una ligera disimilitud (figura 31).

Figura 31. Gráfico de puntajes canónicos de los ejemplares de T. garciabesi.

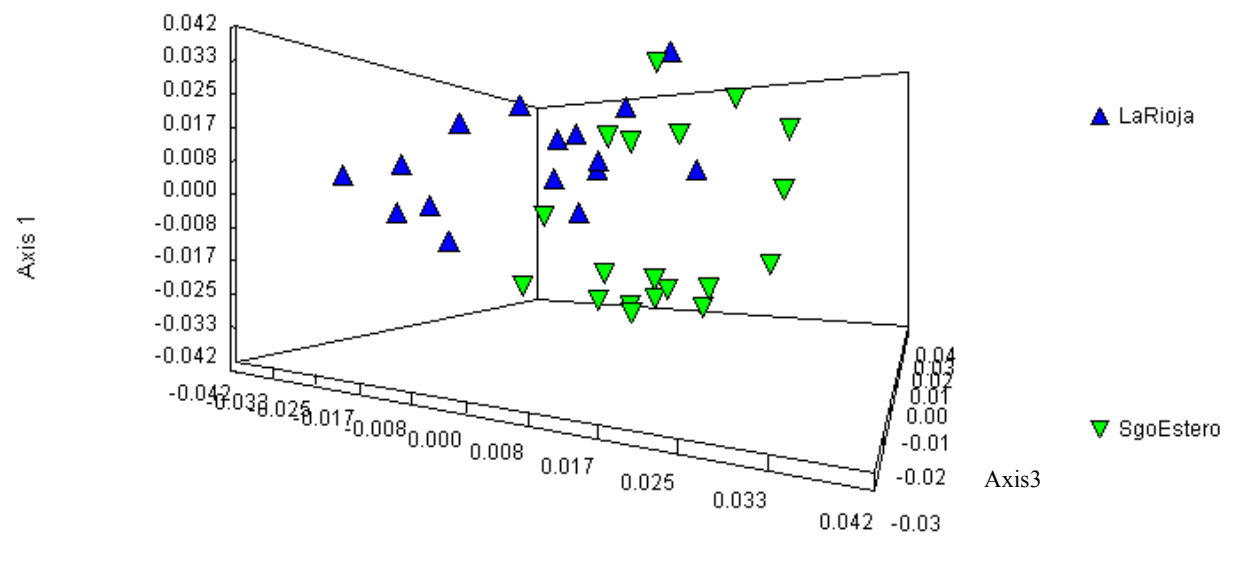

Axis 2

HC con mayor contribución relativa (KIs): 2294, 2700, 2772, 2800, 2875, 2900, 2958, 2974, 2994, 3039, 3091, $3100,3175,3200,3242,3300,3397,3404,3445,3527,3649,3766,3773,3941,4046$

En cuanto a los ejemplares de T. guasayana, se puede observar que sólo los especímenes de Mataral (Bolivia) muestran una ligera diferenciación respecto a los de Argentina y Paraguay que se reúnen en un mismo grupo (figura 32). Los denominados "Tg x Ts" son los que presentan la mayor disimilitud. 
Figura 32. Gráfico de puntajes canónicos de los ejemplares de T. guasayana de Argentina, Bolivia y Paraguay. Se incluyen los ejemplares cruza con $T$. sordida (TgxTs).

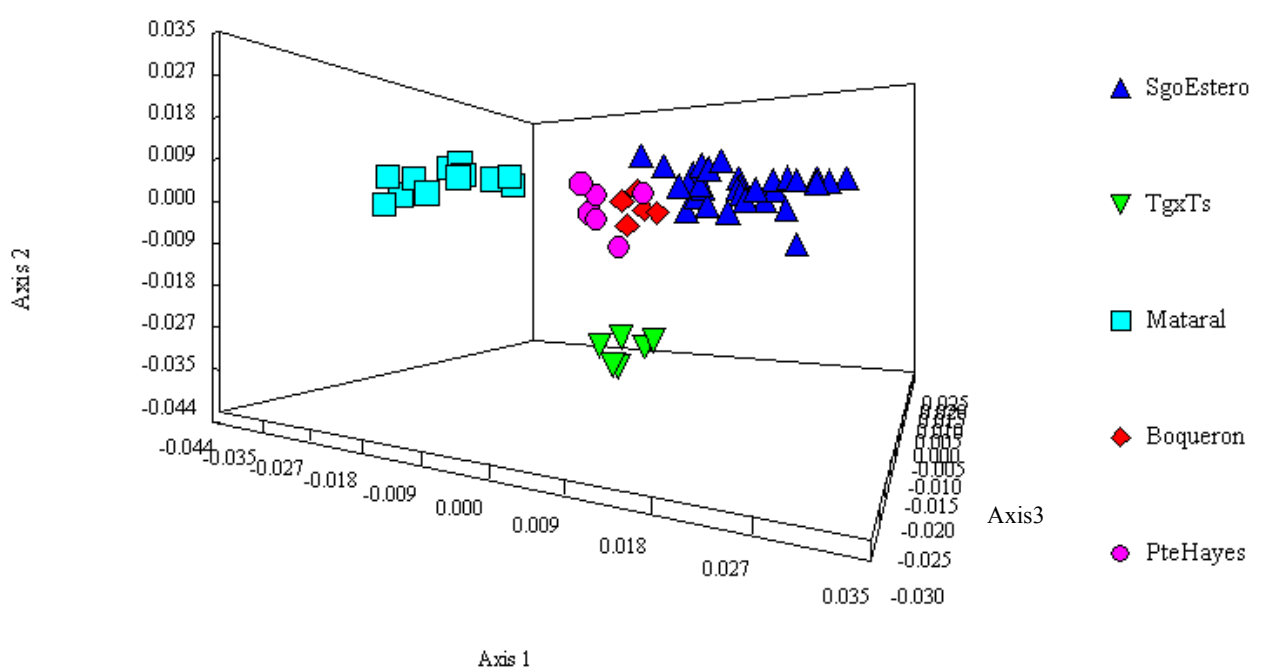

HC con mayor contribución relativa (KIs): 2500, 2700, 2772, 2800, 2900, 2974, 3100, 3156, 3175, 3242, 3372, $3445,3600,3654,3766,3919,3960,3965,4046,4125$

Por último, el PCA de todo el subcomplejo, muestra una clara estructuración en tres grupos principales (figura 33a): uno formado por $T$. guasayana y $T$. patagonica, el más diferenciado, un segundo grupo formado por $T$. garciabesi, estrechamente asociado a $T$. sordida y el tercer grupo formado por $T$. sordida, el cuál a su vez también presenta una separación en dos grupos menores (figura 33b).

Figura 33a. Gráfico de puntajes canónicos de los ejemplares del subcomplejo $T$. sordida de Argentina, Bolivia y Paraguay. Eje 1 en la abcisa y eje 2 en la ordenada

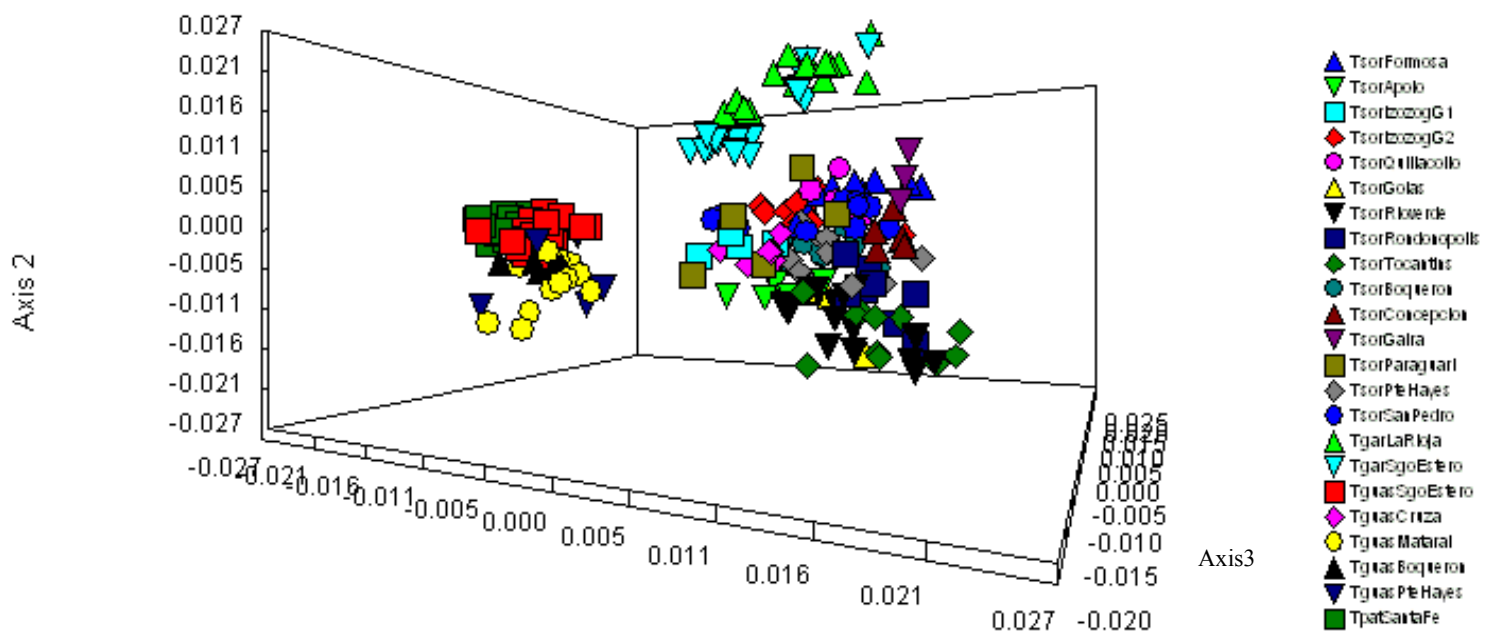


Figura 33b. Gráfico de puntajes canónicos de los ejemplares del subcomplejo T. sordida de Argentina, Bolivia y Paraguay. Eje 1 en la abcisa y eje 3 en la ordenada.

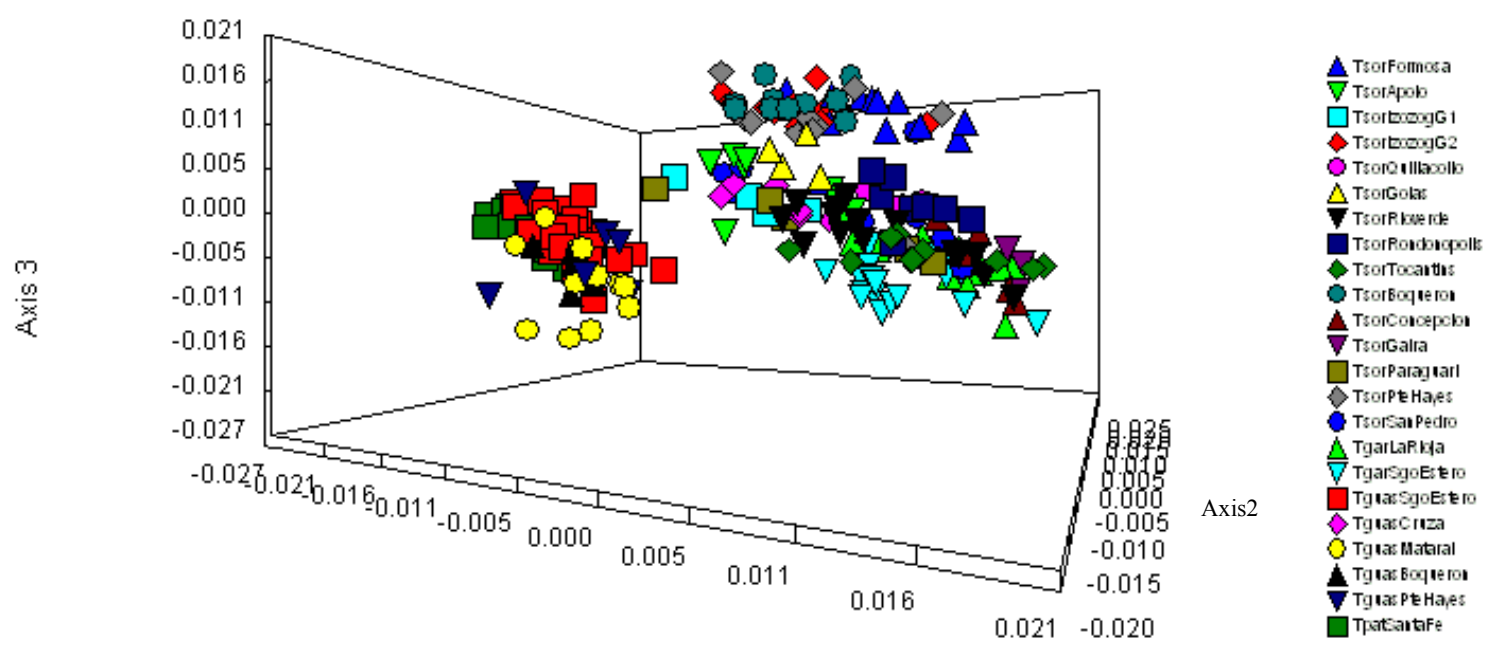

Axis 1

HC con mayor contribución relativa (KIs): 2700, 2772, 2800, 2900, 2974, 3039, 3100, 3175, 3242, 3372, 3378, $3425,3607,3766,3773$

\section{DA sobre T. sordida.}

Debido a que algunas localidades cuentan con un número escaso de insectos colectados (tabla 3), a efectos del DA se agruparon los especímenes de esas localidades tomando en cuenta las relaciones obtenidas en el PCA, de la siguiente manera:

- se eliminaron del análisis los insectos de Quillacollo e Izozog G1 que no mostraron afinidad con ninguna otra población.

- se combinaron las poblaciones de Goias (Brasil) y Apolo (Bolivia), independientemente de su localización geográfica.

- se combinaron las poblaciones del este de Paraguay (San Pedro, Paraguari, Gaira, Concepción)

Se empleó el método de eliminación hacia atrás como mejor criterio de selección de variables, obteniéndose $8 \mathrm{HC}$ como variables que mejor discriminan entre los grupos (KIs 2858, 3175, 3300, 3372, 3378, 3575, 3798, 3823); el $\lambda$ de Wilks resultó altamente significativo $(\mathrm{p} \leq 0.001)$. En la tabla 7 se computan los autovalores, varianzas parcial y acumulada y correlaciones canónicas de las funciones discriminantes (FD) obtenidas. 
Tabla 7. Características de las funciones discriminantes obtenidas en el análisis de $T$. sordida (poblaciones agrupadas).

\begin{tabular}{|l|r|r|r|r|}
\hline Autovalores \\
\hline Función & Autov alor & $\%$ de varianza & $\%$ acumulado & $\begin{array}{c}\text { Correlación } \\
\text { canónica }\end{array}$ \\
\hline 1 & $10.813^{\mathrm{a}}$ & 60.5 & 60.5 & .957 \\
2 & $2.725^{\mathrm{a}}$ & 15.2 & 75.7 & .855 \\
3 & $2.067^{\mathrm{a}}$ & 11.6 & 87.3 & .821 \\
4 & $.988^{\mathrm{a}}$ & 5.5 & 92.8 & .705 \\
5 & $.938^{\mathrm{a}}$ & 5.2 & 98.0 & .696 \\
6 & $.288^{\mathrm{a}}$ & 1.6 & 99.6 & .473 \\
7 & $.055^{\mathrm{a}}$ & .3 & 99.9 & .228 \\
8 & $.010^{\mathrm{a}}$ & .1 & 100.0 & .099 \\
\hline
\end{tabular}

En las tablas 8 y 9 se muestran la matriz de estructura y el valor de las funciones discriminantes (FD) en los centroides de los grupos, mientras que en la figura 34 se grafican los especimenes sobre las tres primeras FD, que acumulan un $87 \%$ de la varianza total.

Tabla 8. Matriz de estructura de las funciones discriminantes obtenidas en el análisis de $T$. sordida (poblaciones agrupadas).

Matriz de estructura

\begin{tabular}{|c|c|c|c|c|c|c|c|c|}
\hline & \multicolumn{8}{|c|}{ Función } \\
\hline & 1 & 2 & 3 & 4 & 5 & 6 & 7 & 8 \\
\hline $\mathrm{KI} 3175$ & $-.645^{\star}$ & .067 & -.366 & .486 & .196 & .291 & .019 & .292 \\
\hline KI3372 & -.107 & .347 & -.218 & $.801^{*}$ & .290 & .036 & -.144 & .268 \\
\hline KI3575 & .438 & -.117 & .165 & .023 & .383 & $.646^{*}$ & -.220 & -.393 \\
\hline KI3378 & -.324 & .513 & .467 & -.116 & .032 & $.550^{*}$ & -.169 & -.259 \\
\hline KI3823 & .111 & -.099 & .374 & .284 & -.153 & -.119 & $.679^{*}$ & -.508 \\
\hline KI2858 & .132 & .162 & -.389 & -.308 & -.303 & .463 & $.635^{\star}$ & -.006 \\
\hline KI3798 & .098 & .097 & -.034 & -.038 & .541 & .144 & .245 & $-.778^{*}$ \\
\hline KI3300 & .031 & .098 & .384 & -.016 & .437 & -.216 & .127 & $.767^{*}$ \\
\hline
\end{tabular}

Correlaciones intra-grupo combinadas entre las variables discriminantes y las funciones discriminantes canónicas tipificadas

Variables ordenadas por el tamaño de la correlación con la función.

*. May or correlación absoluta entre cada variable y cualquier función discriminante.

Tabla 9. Valores de las funciones discriminantes en los centroides de los grupos de T. sordida (poblaciones agrupadas).

Funciones en los centroides de los grupos

\begin{tabular}{|l|r|r|r|r|r|r|r|r|}
\hline & \multicolumn{10}{|c|}{ Función } \\
\cline { 2 - 8 } GRUPO & \multicolumn{1}{|c|}{1} & \multicolumn{1}{c|}{2} & \multicolumn{1}{c|}{3} & \multicolumn{1}{c|}{4} & \multicolumn{1}{c|}{5} & \multicolumn{1}{c|}{6} & \multicolumn{1}{c|}{7} & 8 \\
\hline Formosa & -2.491 & 2.484 & -1.552 & -1.249 & -.081 & -.139 & -.280 & .027 \\
Apolo+Goias & 4.679 & 2.050 & -.893 & 2.088 & .254 & -.126 & -.057 & -.027 \\
Izozog G2 & -5.720 & -1.673 & -1.816 & .867 & 1.082 & .330 & .111 & -.060 \\
Riov erde & 2.703 & -.233 & .279 & -1.010 & 1.324 & -.505 & .248 & -.008 \\
Rondonopolis & 3.247 & .619 & -.298 & -.897 & -.733 & 1.546 & .255 & -.043 \\
Tocantins & 2.218 & -2.197 & 1.039 & -.020 & .739 & .404 & -.464 & .089 \\
Boqueron & -3.667 & 1.350 & 2.017 & .663 & -.146 & .089 & .231 & .204 \\
Con+Gai+Par+SPe & .527 & -1.466 & -.570 & -.001 & -1.402 & -.389 & .046 & .012 \\
PteHayes & -2.292 & .612 & 2.674 & .063 & -.309 & -.102 & -.110 & -.203 \\
\hline
\end{tabular}

Funciones discriminantes canónicas no tipificadas ev aluadas en las medias de los grupos 
Figura 34. Gráfico de puntajes canónicos de los ejemplares de $T$. sordida de Argentina, Bolivia, Brasil y Paraguay (poblaciones agrupadas).
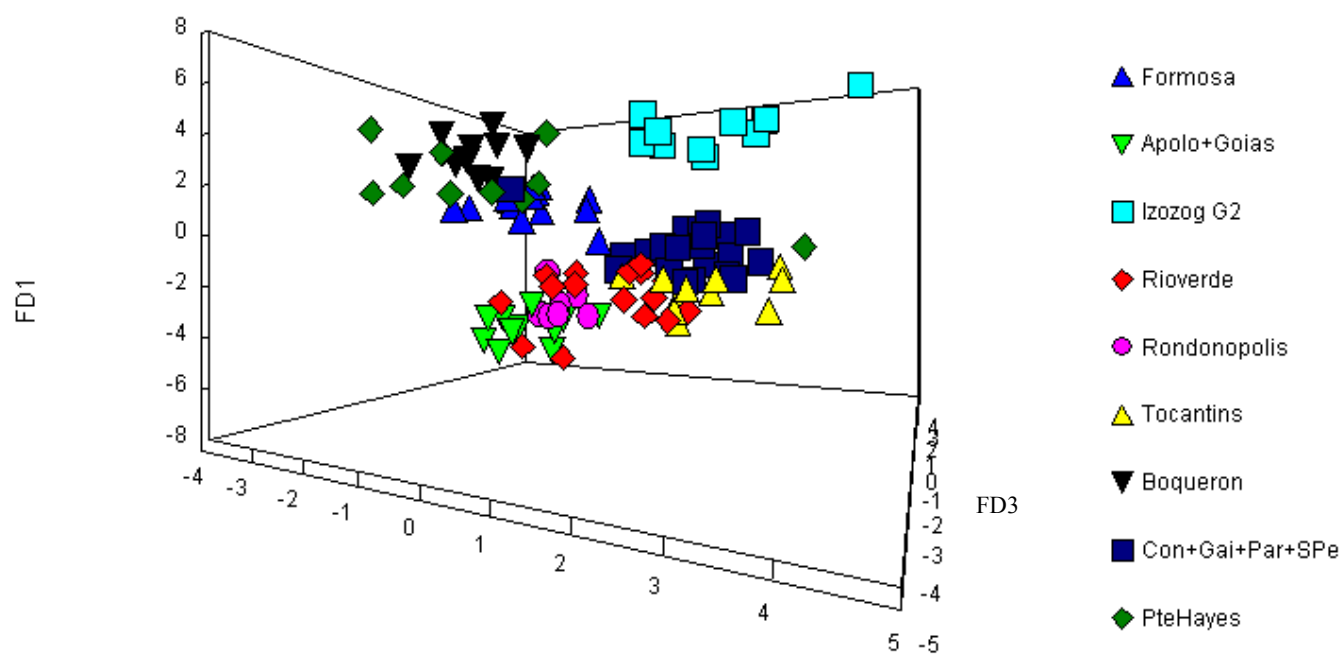

FD2

La FD 1, influenciada principalmente por el HC KI 3175, contribuye a la separación de los dos grupos principales en los que esta especie se divide (grupo 1 y grupo 2). La FD 2, influenciada mayormente por los HC KIs 3372 y 3378, contribuye a la separación de los insectos de Izozog G2 dentro del grupo 2 y de los insectos de Apolo y Goias dentro del grupo 1. La FD 3, influenciada por los HC KIs 2858, 3300 y 3378, contribuye a la separación de los insectos de Formosa (Argentina) dentro del grupo 2 y a los de Tocantins (Brasil) dentro del 1.

Figura 35. Dendrograma basado en la distancia de Mahalanobis entre las poblaciones de $T$. sordida derivado a partir del DA (poblaciones agrupadas)

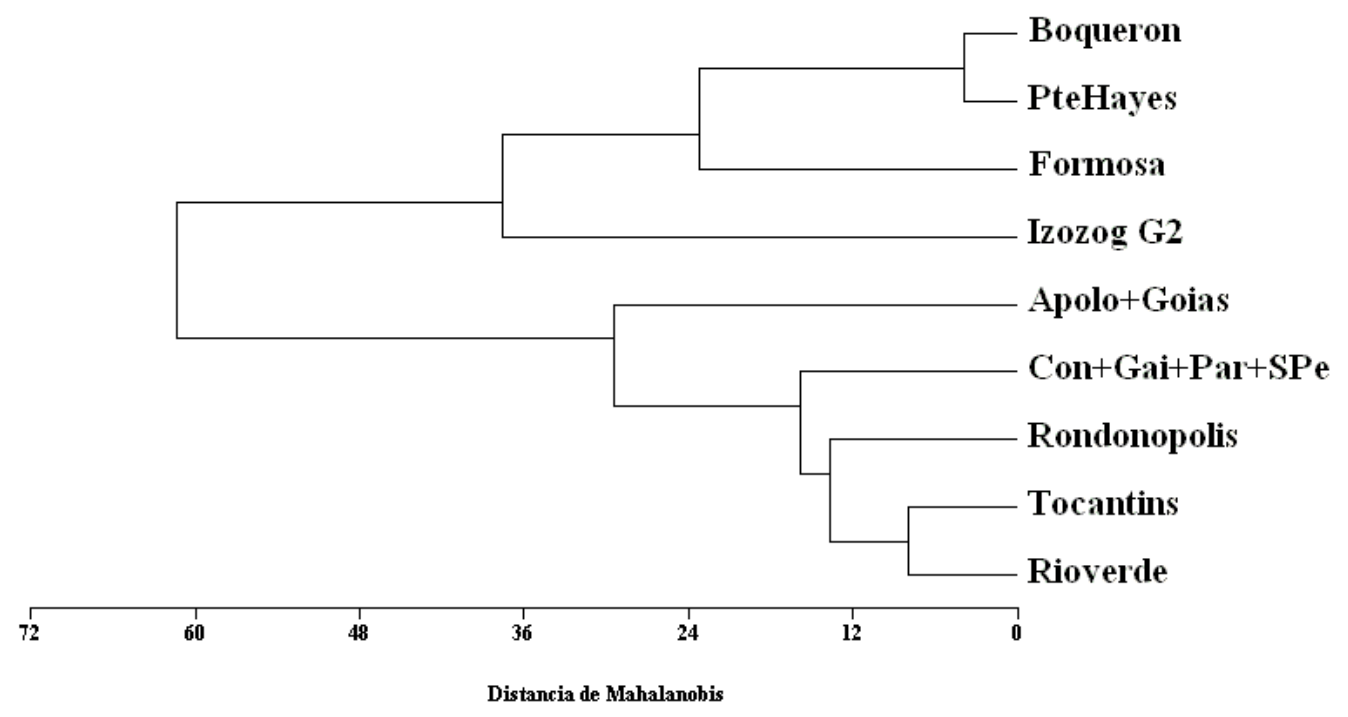


El dendrograma basado en las distancias de Mahalanobis entre los centroides de las poblaciones (figura 35), permite observar con mayor claridad la segregación de éstas en los dos grupos, 1 y 2 . Dentro de cada grupo la poblaciones se reúnen mayormente en base a su proximidad geográfica.

Los resultados de clasificación original y mediante validación cruzada (tabla 10), muestran que las funciones discriminantes obtenidas son consistentes para establecer la estructura poblacional de la especie. Las clasificaciones parciales de las poblaciones muestran errores de clasificación que concuerdan con lo observado en los gráficos sobre las segregación de los ejemplares analizados en dos grupos, es decir hay mayores errores de clasificación entre las poblaciones de un mismo grupo que entre esos grupos obtenidos.

Tabla 10. Resultados de clasificación de los grupos de T. sordida (poblaciones agrupadas) (\%)

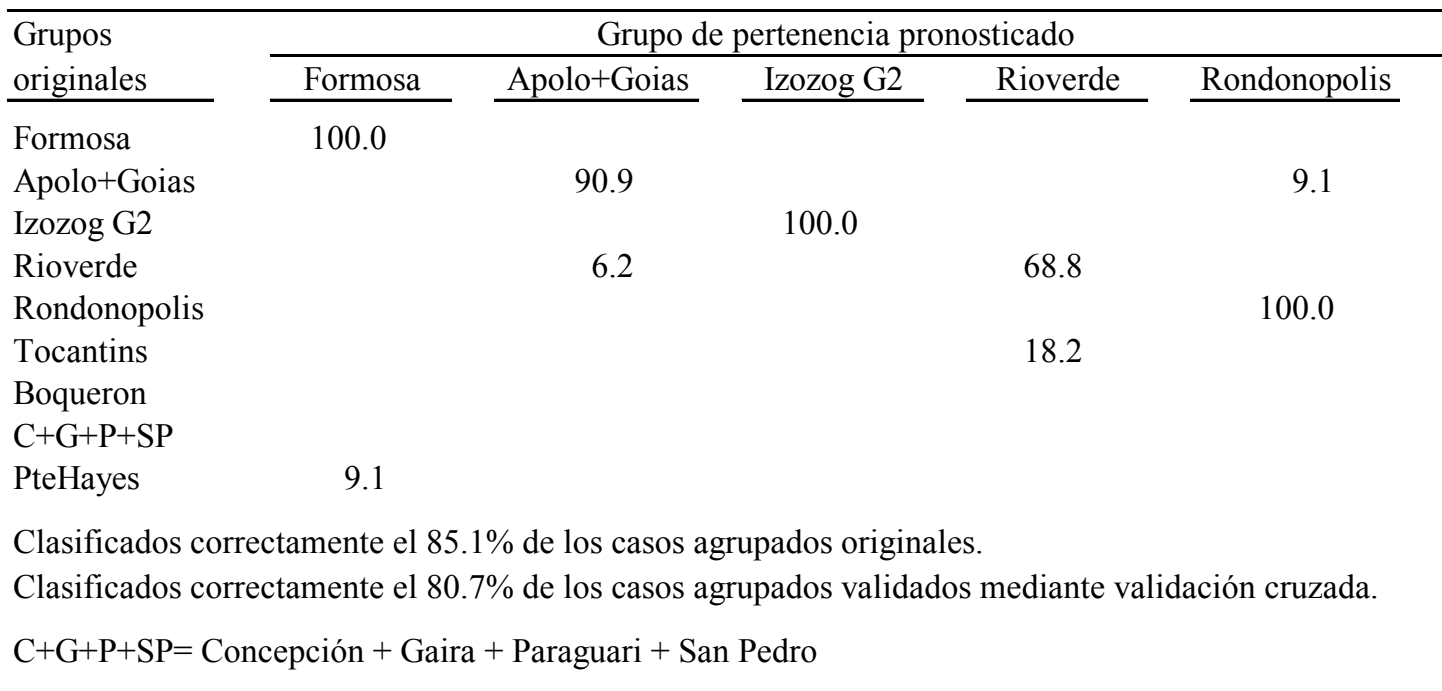

\begin{tabular}{|c|c|c|c|c|}
\hline \multirow{2}{*}{$\begin{array}{l}\text { Grupos } \\
\text { originales }\end{array}$} & \multicolumn{4}{|c|}{ Grupo de pertenencia pronosticado } \\
\hline & Tocantins & Boqueron & $\mathrm{C}+\mathrm{G}+\mathrm{P}+\mathrm{SP}$ & PteHaye \\
\hline & 7.7 \\
\hline \multicolumn{5}{|l|}{ Apolo+Goias } \\
\hline \multicolumn{5}{|l|}{ Izozog G2 } \\
\hline Rioverde & 12.5 & & 12.5 & \\
\hline \multicolumn{5}{|l|}{ Rondonopolis } \\
\hline Tocantins & 63.6 & & 18.2 & \\
\hline Boqueron & & 70.0 & & 30.0 \\
\hline $\mathrm{C}+\mathrm{G}+\mathrm{P}+\mathrm{SP}$ & 8.7 & & 87.0 & 4.3 \\
\hline PteHayes & 9.1 & 36.4 & & 45.5 \\
\hline \multicolumn{5}{|c|}{$\begin{array}{l}\text { Clasificados correctamente el } 85.1 \% \text { de los casos agrupados originales. } \\
\text { Clasificados correctamente el } 80.7 \% \text { de los casos agrupados validados mediante } \\
\text { validación cruzada. }\end{array}$} \\
\hline \multicolumn{5}{|c|}{$\mathrm{C}+\mathrm{G}+\mathrm{P}+\mathrm{SP}=$ Concepción + Gaira + Paraguari + San Pedro } \\
\hline
\end{tabular}


A continuación, se realizó el análisis de todas las localidades por separado, obteniéndose $9 \mathrm{HC}$ como variables que mejor discriminan entre las poblaciones (KIs 2858, $3175,3187,3300,3372,3378,3575,3800,3823)$, con un $\lambda$ de Wilks altamente significativo ( $\mathrm{p} \leq 0.001)$. En la tabla 11 se computan los autovalores, varianzas y correlaciones canónicas de las funciones discriminantes.

Tabla 11. Características de las funciones discriminantes obtenidas en el análisis de $T$. sordida.

\begin{tabular}{|l|r|r|r|r|}
\hline Autoval ores \\
\hline Función & Autov alor & $\%$ de varianza & $\%$ acumulado & $\begin{array}{c}\text { Correlación } \\
\text { canónica }\end{array}$ \\
\hline 1 & $13,666^{\mathrm{a}}$ & 47,6 & 47,6 &, 965 \\
2 & $7,534^{\mathrm{a}}$ & 26,2 & 73,8 &, 940 \\
3 & $2,605^{\mathrm{a}}$ & 9,1 & 82,9 &, 850 \\
4 & $2,012^{\mathrm{a}}$ & 7,0 & 89,9 &, 817 \\
5 & $1,337^{\mathrm{a}}$ & 4,7 & 94,5 &, 756 \\
6 &, $783^{\mathrm{a}}$ & 2,7 & 97,3 &, 663 \\
7 &, $574^{\mathrm{a}}$ & 2,0 & 99,3 &, 604 \\
8 &, $160^{\mathrm{a}}$ &, 6 & 99,8 &, 371 \\
9 &, $054^{\mathrm{a}}$ &, 2 & 100,0 &, 227 \\
\hline
\end{tabular}

En las tablas 12 y 13 se muestran la matriz de estructura y el valor de las funciones discriminantes en los centroides de los grupos, mientras que en las figuras 36 a y b se grafican los especimenes sobre las tres primeras funciones discriminantes, que acumulan un $83 \%$ de la varianza total.

Tabla 12. Matriz de estructura de las funciones discriminantes obtenidas en el análisis de $T$. sordida.

Matriz de estructura

\begin{tabular}{|c|c|c|c|c|c|c|c|c|c|}
\hline & \multicolumn{9}{|c|}{ Función } \\
\hline & 1 & 2 & 3 & 4 & 5 & 6 & 7 & 8 & 9 \\
\hline $\mathrm{KI} 3175$ & ,656* & , 117 &,- 276 &,- 284 & ,473 &,- 034 &,- 029 &,- 067 & ,411 \\
\hline KI2858 &,- 125 & , 190 &,$- 775^{\star}$ & 149 & ,013 & ,476 & ,291 & ,019 &,- 116 \\
\hline $\mathrm{KI} 3372$ & 108 &,- 138 &,- 047 &,- 328 &, $780^{*}$ &,- 293 &,- 154 & ,049 &, 373 \\
\hline KI3575 &,- 327 & ,222 &, 447 &,- 161 & ,310 & ,274 &, $578^{*}$ &,- 290 &,- 171 \\
\hline KI3187 & ,343 &,- 398 &,- 217 &,- 125 & ,066 & ,163 & ,460 &, $553^{*}$ &,- 337 \\
\hline KI3378 & ,273 &,- 351 & ,221 & ,418 & ,349 & ,334 & ,296 &,$- 504^{*}$ &,- 061 \\
\hline KI3798 &,- 052 & ,029 & ,208 &,- 296 & ,310 & ,475 & ,055 &,- 152 &,$- 720^{*}$ \\
\hline KI3823 &,- 047 & ,321 & ,023 & ,417 & ,327 & 018 &,- 244 & ,234 &,$- 706^{\star}$ \\
\hline KI3300 &,- 060 &,- 151 & ,321 & ,135 &, 076 & ,287 &,- 368 & ,469 & ,639* \\
\hline
\end{tabular}

Correlaciones intra-grupo combinadas entre las variables discriminantes y las funciones discriminantes canónicas tipif icadas

Variables ordenadas por el tamaño de la correlación con la función.

*. May or correlación absoluta entre cada variable y cualquier función discriminante. 
Tabla 13. Valores de las funciones discriminantes en los centroides de los grupos de $T$. sordida.

Funciones en los centroides de los grupos

\begin{tabular}{|c|c|c|c|c|c|c|c|c|c|}
\hline \multirow[b]{2}{*}{ GRUPO } & \multicolumn{9}{|c|}{ Función } \\
\hline & 1 & 2 & 3 & 4 & 5 & 6 & 7 & 8 & 9 \\
\hline Formosa & 2,499 & $-3,926$ & $-1,596$ &,- 771 &,- 302 & ,575 & 850 & ,236 &,- 179 \\
\hline Apolo & $-4,978$ & $-1,280$ &,- 541 &,- 483 & 2,422 & $-1,095$ &, 512 & ,132 & ,124 \\
\hline Izozog G1 & $-3,555$ & 1,737 & $-5,109$ & 2,548 &,- 635 & ,818 & $-1,194$ &, 577 & ,377 \\
\hline Izozog G2 & 6,162 & ,401 &,- 302 & $-2,248$ &,- 057 &,- 110 &,- 394 &,- 067 & ,238 \\
\hline Quillacollo & 5,883 & 10,623 &,- 888 & ,493 & 1,892 & ,845 & 695 & ,028 &,- 274 \\
\hline Goias & $-4,969$ &,- 443 &,- 357 & $-2,141$ & 2,773 &,- 601 & $-1,064$ & ,208 &,- 258 \\
\hline Riov erde & $-3,073$ & ,128 & 1,177 &,- 738 &,- 559 & 1,237 &,- 711 &, 036 &,- 072 \\
\hline Rondonopolis & $-3,662$ & ,182 &,- 686 &, 571 & ,052 & 813 & 1,259 &,- 914 & , 145 \\
\hline Tocantins & $-1,973$ & 1,649 & 2,265 &,- 263 &,- 581 &,- 077 & 678 & ,404 & ,237 \\
\hline Boqueron & 3,573 & $-1,997$ & ,786 & 1,856 & 1,058 & ,015 &,- 586 &,- 144 & ,238 \\
\hline Concepcion &,- 946 & 1,820 &,- 167 & ,745 & $-1,245$ & $-1,111$ & ,758 & ,727 &,- 217 \\
\hline Gaira & $-1,592$ & 1,175 & $-1,452$ & ,054 &,- 541 &,- 460 &,- 987 &,- 602 &,- 645 \\
\hline Paraguari &,- 243 & ,927 &, 572 &,- 803 & $-1,013$ & $-1,250$ &,- 330 &,- 602 &,- 052 \\
\hline PteHayes & 2,304 & $-1,610$ & 1,657 & 2,174 & ,253 &,- 124 &,- 107 & ,037 &,- 235 \\
\hline SanPedro &,- 505 & ,668 &,- 874 & ,234 & $-1,405$ & $-1,390$ &,- 236 &,- 200 & 033 \\
\hline
\end{tabular}

Funciones discriminantes canónicas no tipificadas ev aluadas en las medias de los grupos

Figura 36a. Gráfico de puntajes canónicos de los ejemplares de T. sordida de Argentina, Bolivia, Brasil y Paraguay. Abscisa: FD 1, ordenada: FD 2.
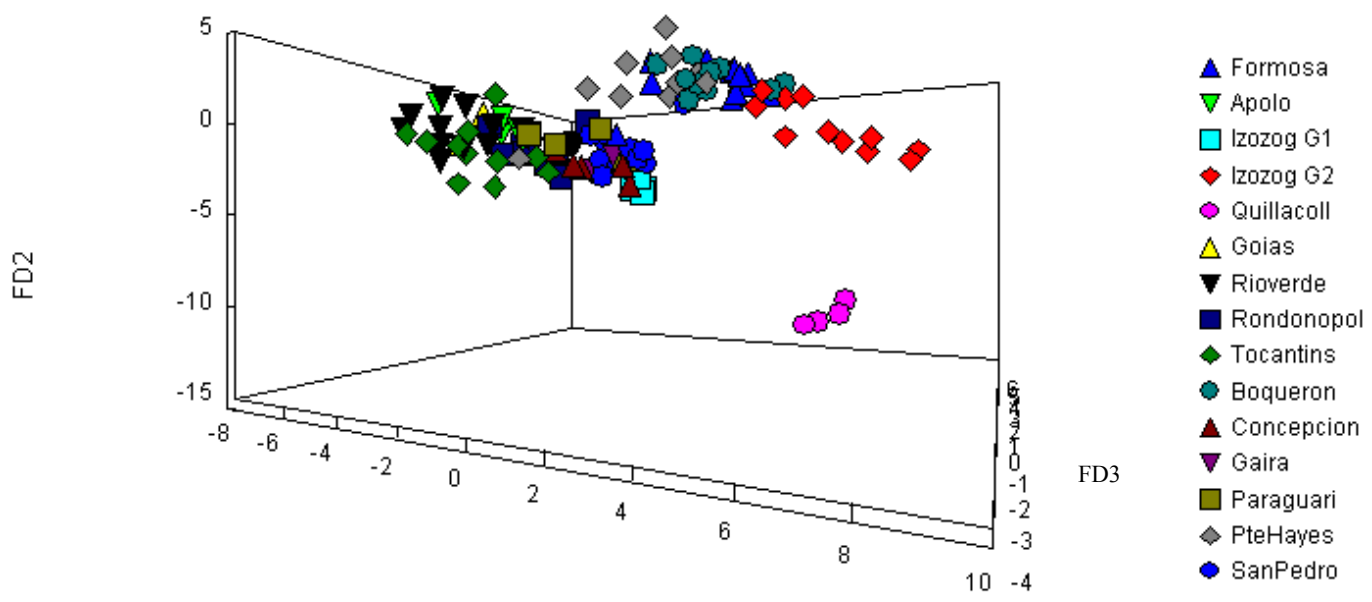

FD1 
Figura 36b. Gráfico de puntajes canónicos de los ejemplares de T. sordida de Argentina, Bolivia, Brasil y Paraguay. Abscisa: FD 1, ordenada: FD 3.

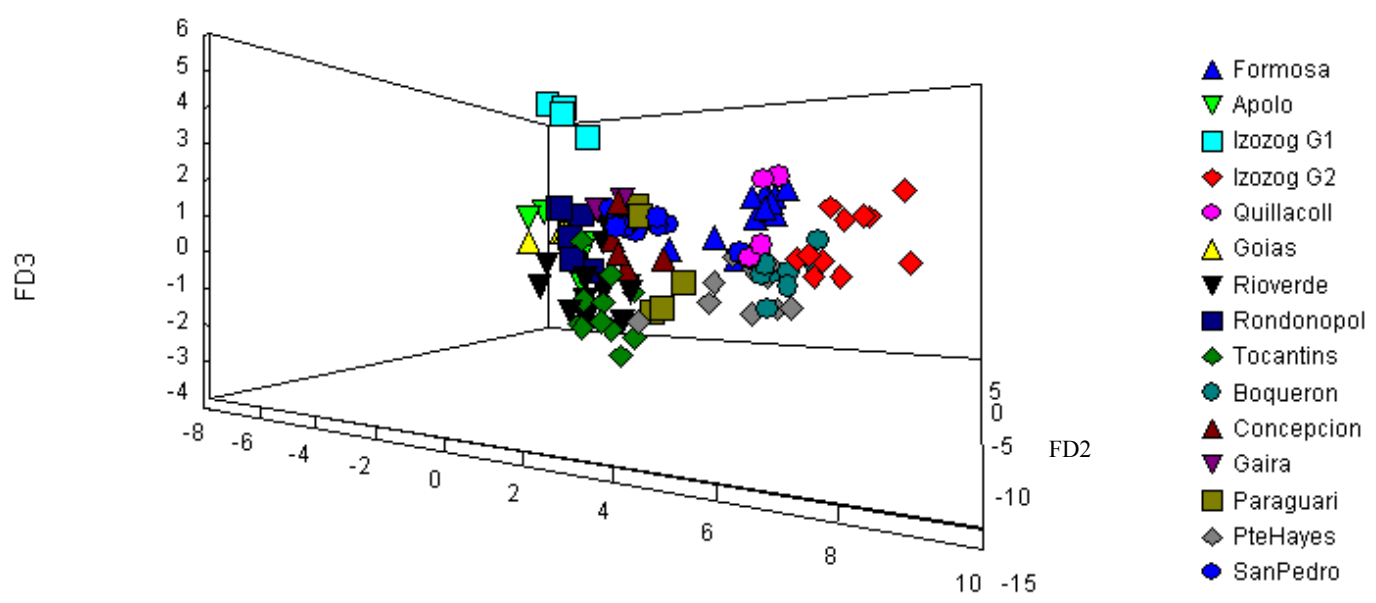

FD1

La FD1, influenciada principalmente por el HC KI 3175 y en menor medida por los HC KIs 3187, 3378 y 3575, contribuye a la separación de los grupos 1 y 2, adoptando el grupo 2 valores de los centroides positivos mientras que el 1 adopta valores negativos. La FD 2 influenciada mayormente por los HC KIs 3187, 3378 y 3823, contribuye a la separación de los insectos de Quillacollo y también de los grupos 1 y 2. La FD 3, influenciada principalmente por los HC KIs 2858, 3300 y 3175 separa a los insectos de Izozog G1.

El dendrograma basado en la distancia de Mahalanobis entre los centroides de las poblaciones (figura 37) muestra que los insectos mas diferenciados son los de la región de Quillacollo, el resto se segrega en dos grupos principales, también claramente diferenciados: el grupo 1 que comprende a todas las poblaciones de Brasil, las de la región oriental de Paraguay, la población andina de Apolo y los especimenes de Izozog (Bolivia) designados como G1; y el grupo 2 formado por los especimenes de Argentina (Formosa), los de la región occidental de Paraguay y los de Izozog (Bolivia) designados como G2 . Dentro de los grupos, los insectos muestran un grado de similitud que coincide en términos generales con su proximidad geográfica, sin embargo los insectos de la región boliviana andina de Apolo muestran una gran similitud con los de el este de Brasil (Goias). 
Figura 37. Dendrograma basado en la distancia de Mahalanobis entre las poblaciones de $T$. sordida derivado a partir del DA

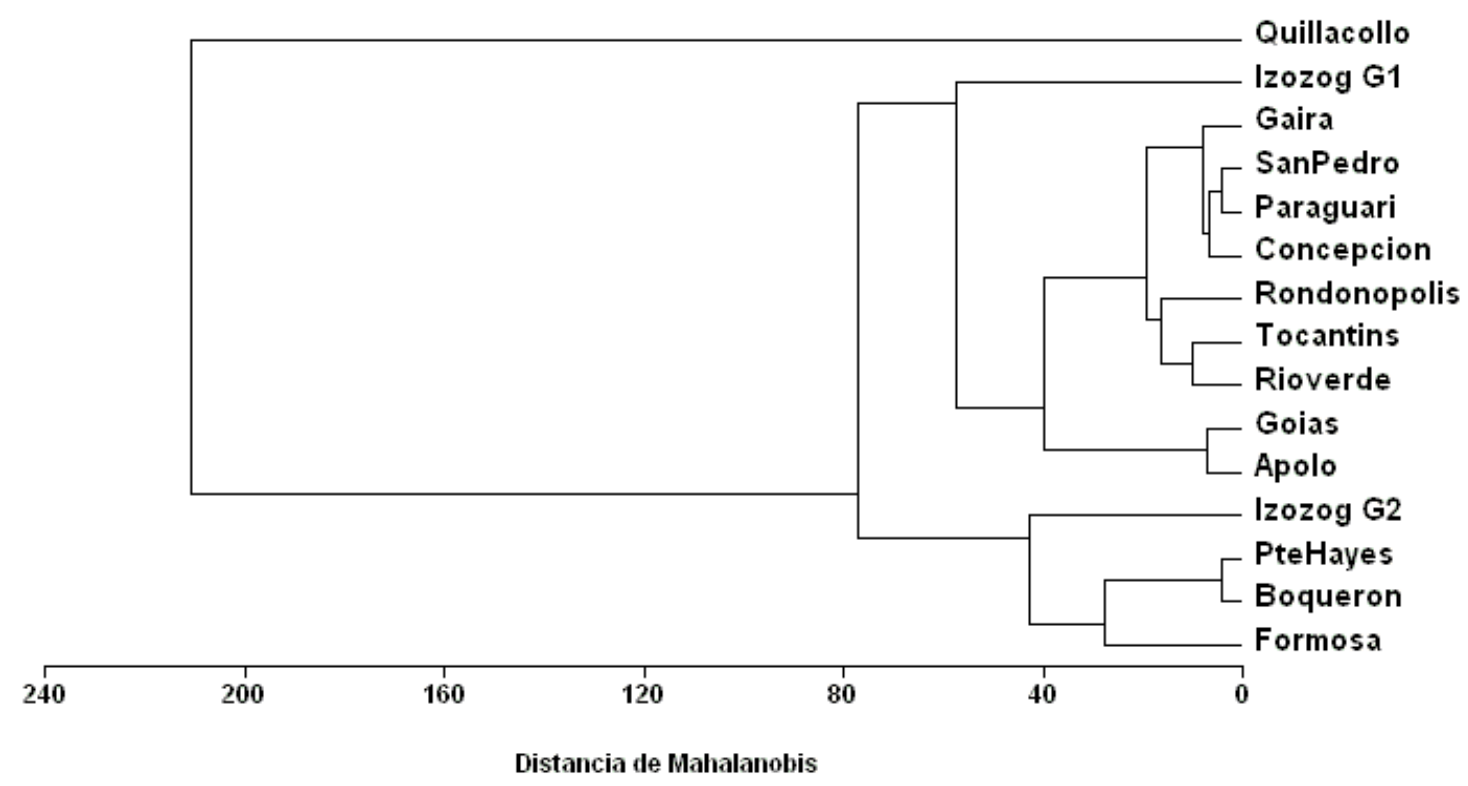

Tabla 14. Resultados de clasificación de los grupos de T. sordida (\%).

\begin{tabular}{|c|c|c|c|c|c|}
\hline & & Grup & ertenencia $\mathrm{p}$ & sticado & \\
\hline & Formosa & Apolo & Izozog G1 & Izozog G2 & Quillacollo \\
\hline Formosa & 92.3 & & & & \\
\hline Apolo & & 71.4 & & & \\
\hline Izozog G1 & & & 100.0 & & \\
\hline Izozog G2 & & & & 100.0 & \\
\hline Quillacollo & & & & & 100.0 \\
\hline Goias & & 25.0 & & & \\
\hline Rioverde & & 6.3 & & & \\
\hline Rondonopolis & & & & & \\
\hline Tocantins & & & & & \\
\hline Boqueron & & & & & \\
\hline Concepcion & & & & & \\
\hline Gaira & & & & & \\
\hline Paraguari & & & & & \\
\hline PteHayes & 9.1 & & & & \\
\hline SanPedro & & & & & \\
\hline
\end{tabular}




\begin{tabular}{|c|c|c|c|c|c|}
\hline \multirow{2}{*}{ 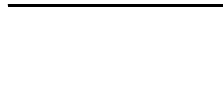 } & \multicolumn{5}{|c|}{ Grupo de pertenencia pronosticado } \\
\hline & Goias & Rioverde & Rondonopolis & Tocantins & Boqueron \\
\hline \multicolumn{6}{|l|}{ Formosa } \\
\hline Apolo & & 14.3 & 14.3 & & \\
\hline \multicolumn{6}{|l|}{ Izozog G1 } \\
\hline \multicolumn{6}{|l|}{ Izozog G2 } \\
\hline \multicolumn{6}{|l|}{ Quillacollo } \\
\hline Goias & 75.0 & & & & \\
\hline Rioverde & & 68.8 & & 12.5 & \\
\hline Rondonopolis & & & 100.0 & & \\
\hline Tocantins & & 18.2 & & 63.6 & \\
\hline Boqueron & & & & & 60.0 \\
\hline \multicolumn{6}{|l|}{ Concepcion } \\
\hline \multicolumn{6}{|l|}{ Gaira } \\
\hline Paraguari & & & & 40.0 & \\
\hline PteHayes & & & & 9.1 & 45.5 \\
\hline \multicolumn{6}{|l|}{ SanPedro } \\
\hline Clasificados ce & amente & $\%$ de los ca & agrupados origir & & \\
\hline Clasificados ce & amente & $\%$ de los ca & agrupados valid & mediante & ción cruz \\
\hline
\end{tabular}

\begin{tabular}{|c|c|c|c|c|c|}
\hline \multirow{2}{*}{ 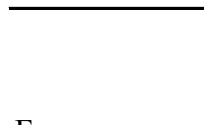 } & \multicolumn{5}{|c|}{ Grupo de pertenencia pronosticado } \\
\hline & Concepcion & Gaira & Paraguari & PteHayes & SanPedro \\
\hline Formosa & & & & 7.7 & \\
\hline Apolo & & & & & \\
\hline Izozog G1 & & & & & \\
\hline Izozog G2 & & & & & \\
\hline Quillacollo & & & & & \\
\hline Goias & & & & & \\
\hline Rioverde & & & 12.5 & & \\
\hline Rondonopolis & & & & & \\
\hline Tocantins & 9.1 & 9.1 & & & \\
\hline Boqueron & & & & 40.0 & \\
\hline Concepcion & 60.0 & & & & 40.0 \\
\hline Gaira & & 33.3 & & & 66.7 \\
\hline Paraguari & & & 20.0 & & 40.0 \\
\hline PteHayes & & & & 36.4 & \\
\hline SanPedro & & & 10.0 & 10.0 & 80.0 \\
\hline
\end{tabular}

Los resultados de clasificación mediante validación cruzada (tabla 14) muestran que un $72 \%$ de los especímenes resultaron correctamente clasificados, lo que por comparación con el resultado de clasificación original ( $82 \%$ ), sugiere que las funciones discriminantes son robustas para clasificar las poblaciones de las especie. La clasificación de los grupos estudiados coincide con lo mostrado en los gráficos; los errores de clasificación parciales 
muestran un grado variable de superposición en las poblaciones dentro de cada grupo 1 o 2 , pero muy poca entre los grupos.

\section{DA sobre T. garciabesi.}

Para la clasificación de esta especie se computaron con el método de eliminación hacia atrás $10 \mathrm{HC}$ (KIs 2700, 2858, 2900, 2951, 2958, 3242, 3357, 3372, 3575, 3866), a partir de los cuales se obtuvo una función discriminante con un $\lambda$ de Wilks significativo $(\mathrm{p} \leq 0.001)$. Debido a que se obtuvo una sola función discriminante, se omite la tabla con autovalores y varianza acumulada para esta especie. Las tablas 15 y 16 muestran la matriz de estructura y el valor de la función discriminante en los centroides de los dos grupos. Los HC con KI 2858 y 3357 son los que mas contribuyen a la discriminación entre las dos poblaciones analizadas.

Tabla 15. Matriz de estructura de la función discriminante obtenida en el análisis de $T$. garciabesi.

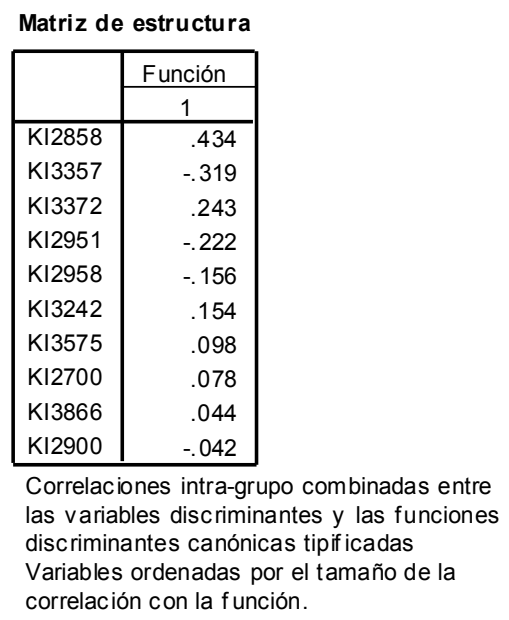

Tabla 16. Valores de las función discriminante en los centroides de los grupos de $T$. garciabesi.

unciones en los centroides de los grupo:

\begin{tabular}{|l|r|}
\hline \multirow{2}{*}{ GRUPO } & Función \\
\cline { 2 - 2 } & 1 \\
\hline La Rioja & 2.653 \\
Sgo Estero & -2.234 \\
\hline
\end{tabular}

Funciones discriminantes canónicas no tipificadas ev aluadas en las medias de los grupos

La clasificación de los especimenes mediante validación cruzada (tabla 17) muestra que existe un ligero error en la clasificación, resultado de la similitud de las poblaciones. 
Tabla 17. Resultados de clasificación de los grupos de T. garciabesi (\%).

\begin{tabular}{|c|c|c|}
\hline \multirow{2}{*}{$\begin{array}{l}\text { Grupos } \\
\text { originales }\end{array}$} & \multicolumn{2}{|c|}{ Grupo de pertenencia pronosticado } \\
\hline & La Rioja & Sgo Estero \\
\hline La Rioja & 87.5 & 12.5 \\
\hline Sgo Estero & 5.3 & 94.7 \\
\hline \multicolumn{3}{|c|}{$\begin{array}{l}\text { Clasificados correctamente el } 100.0 \% \text { de los casos agrupados originale } \\
\text { Clasificados correctamente el } 91.4 \% \text { de los casos agrupados validados } \\
\text { mediante validación cruzada. }\end{array}$} \\
\hline
\end{tabular}

DA sobre T. guasayana.

Cinco HC fueron computados tras la aplicación del método de eliminación hacia atrás (KIs 2974, 3351, 3725, 3766, 3798), obteniéndose cuatro funciones discriminantes con un $\lambda$ de Wilks altamente significativo $(\mathrm{p} \leq 0.001)$; las características de esas funciones se muestran en la tabla 18.

Tabla 18. Características de las funciones discriminantes del análisis de T. guasayana.

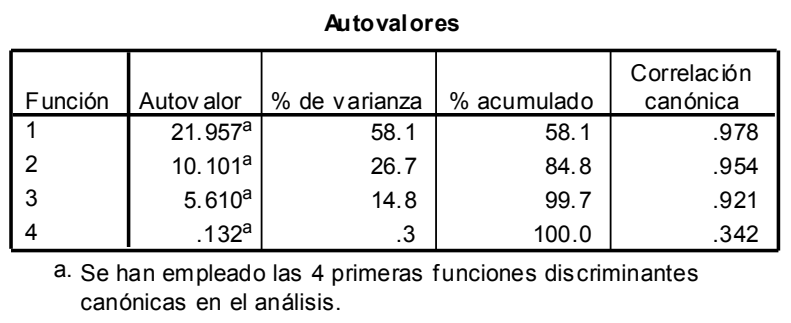

En las tablas 19 y 20 se muestran la matriz de estructura y los valores de las funciones discriminantes en los centroides de los grupos.

Tabla 19. Matriz de estructura de las funciones discriminantes obtenidas en el análisis de $T$. guasayana.

Matriz de estructura

\begin{tabular}{|l|c|c|c|c|}
\hline & \multicolumn{4}{|c|}{ Función } \\
\cline { 2 - 5 } & 1 & 2 & 3 & 4 \\
\hline KI3725 & $.707^{*}$ & .237 & .208 & -.496 \\
KI3766 & $-.276^{\star}$ & -.152 & .204 & -.196 \\
KI3798 & .062 & .267 & $-.428^{\star}$ & -.318 \\
KI3351 & .305 & -.439 & -.101 & $.707^{*}$ \\
KI2974 & .052 & .585 & .484 & $.634^{*}$ \\
\hline
\end{tabular}

Correlaciones intra-grupo combinadas entre las variables discriminantes y las funciones discriminantes canónicas tipif icadas

Variables ordenadas por el tamaño de la correlación con la función.

*. May or correlación absoluta entre cada variable y cualquier función discriminante. 
Tabla 20. Valores de las funciones discriminantes en los centroides de los grupos de $T$. guasayana.

Funciones en los centroides de los grupos

\begin{tabular}{|l|r|r|r|r|}
\hline & \multicolumn{4}{|c|}{ Función } \\
\cline { 2 - 5 } GRUPOS & \multicolumn{1}{|c|}{1} & \multicolumn{1}{|c|}{2} & \multicolumn{1}{c|}{3} \\
\hline SgoEstero & -3.067 & .111 & 1.502 & .001 \\
TgxTs & 7.743 & 7.564 & .302 & -.024 \\
Mataral & 6.488 & -4.295 & .133 & -.016 \\
Boqueron & -2.769 & .218 & -4.847 & -.871 \\
PteHayes & -2.055 & .253 & -4.541 & .774 \\
\hline
\end{tabular}

Funciones discriminantes canónic as no tipificadas ev aluadas en las medias de los grupos

En las figuras 38 a y b se grafican las poblaciones sobre las tres primeras FD que acumulan casi el $100 \%$ de la variación total.

Figura 38a. Gráfico de puntajes canónicos de los ejemplares de T. guasayana, incluidos los insectos Tg x Ts. Abscisa: FD 1, ordenada: FD 2.

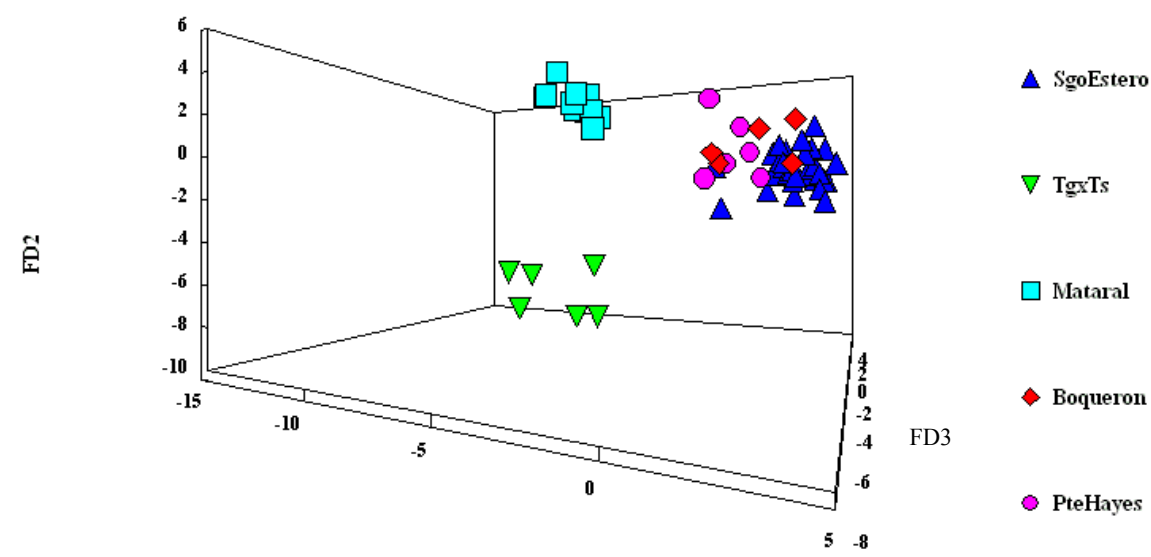

FD1

Figura 38b. Gráfico de puntajes canónicos de los ejemplares de T. guasayana, incluidos los insectos Tg x Ts. Abscisa: FD 1, ordenada: FD 3.

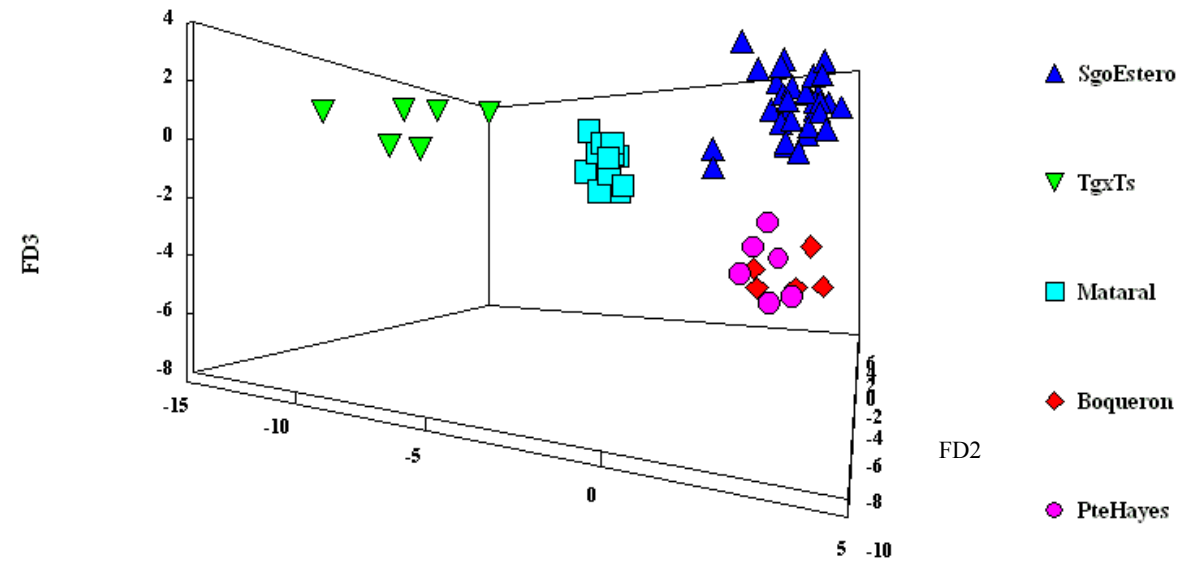

FDl 
La FD 1, influenciada principalmente por el HC KI 3725 y en menor medida también por los HC KIs 3351 y 3766, contribuye a la separación de los insectos de Mataral y los TgxTs (valores positivos de la FD) respecto a los de Paraguay y Argentina (valores negativos). La FD 2, influenciada principalmente por los HC KIs 2974 y 3351, contribuye a la separación de los insectos de Mataral respecto a los TgxTs en mayor medida y en menor medida al resto. La FD 3, influenciada mayormente por los HC KIs 3798 y 2974, diferencia principalmente a los insectos de Argentina respecto a los de Paraguay. Por último la FD 4, influenciada en mayor medida por los HC KIs 3351 y 2974, contribuye a la diferenciación entre los especimenes de Boquerón y los de Presidente Hayes.

Al analizar los resultados de clasificación (tabla 21), se ve que si bien el porcentaje de individuos correctamente clasificados tras la validación cruzada de los casos es alto, existe una mala separación de los especimenes paraguayos (alto error de clasificación), producto de su alta similitud.

Tabla 21. Resultados de clasificación de los grupos de $T$. guasayana, incluidos los especímenes Tg x Ts. (\%)

\begin{tabular}{|c|c|c|c|c|c|}
\hline \multirow{2}{*}{$\begin{array}{l}\text { Grupo } \\
\text { original }\end{array}$} & \multicolumn{5}{|c|}{ Grupo de pertenencia pronosticado } \\
\hline & Sgo Estero & TgxTs & Mataral & Boqueron & Pte Hayes \\
\hline Sgo Estero & 96.9 & & & & 3.1 \\
\hline TgxTs & & 100.0 & & & \\
\hline Mataral & & & 100.0 & & \\
\hline Boqueron & & & & 60.0 & 40.0 \\
\hline PteHayes & & & & 16.7 & 83.3 \\
\hline
\end{tabular}

DA sobre el subcomplejo T. sordida.

En base a los resultados previsualizados en los PCA, y los resultados de los DA para cada especie, el análisis conjunto de todos las especies del subcomplejo, se llevo a cabo en primer término uniendo ciertas poblaciones con bajo número de insectos colectados a fin de incrementar el $\mathrm{n}$ de esas muestras, y eliminando aquellas poblaciones que no podían ser reunidas con ninguna otra. De esta manera:

- se eliminaron los ejemplares de T. sordida de Quillacollo e Izozog G1 y los ejemplares cruza entre T. sordida y T. guasayana (TgxTs) 
- se unificaron las poblaciones de T. guasayana de Boquerón y Presidente Hayes, las de $T$. sordida de Apolo con las de Goias y las de la región oriental de Paraguay (Concepción, San Pedro, Paraguari y Gaira).

Como resultado del DA, siete $\mathrm{HC}$ fueron seleccionados como variables que mejor discriminan entre los grupos analizados (KIs 2974, 2994, 3175, 3285, 3372, 3725, 3971), con un $\lambda$ de Wilks altamente significativo $(\mathrm{p} \leq 0.001)$. Las características de las funciones discriminantes obtenidas se muestran en la tabla 22 .

Tabla 22. Características de las funciones discriminantes obtenidas en el análisis del subcomplejo T. sordida (poblaciones agrupadas).

\begin{tabular}{|l|r|r|r|r|}
\hline \multicolumn{5}{|c|}{ Autoval ores } \\
\hline Función & Autov alor & $\%$ de varianza & $\%$ acumulado & $\begin{array}{c}\text { Correlación } \\
\text { canónica }\end{array}$ \\
\hline 1 & $26.833^{\mathrm{a}}$ & 52.1 & 52.1 & .982 \\
2 & $14.353^{\mathrm{a}}$ & 27.9 & 79.9 & .967 \\
3 & $4.823^{\mathrm{a}}$ & 9.4 & 89.3 & .910 \\
4 & $2.084^{\mathrm{a}}$ & 4.0 & 93.3 & .822 \\
5 & $1.840^{\mathrm{a}}$ & 3.6 & 96.9 & .805 \\
6 & $.940^{\mathrm{a}}$ & 1.8 & 98.7 & .696 \\
7 & $.658^{\mathrm{a}}$ & 1.3 & 100.0 & .630 \\
\hline
\end{tabular}

La matriz de estructura y los valores de las funciones discriminantes en los centroides de los grupos se muestran en las tablas 23 y 24 .

Tabla 23. Matriz de estructura de las funciones discriminantes obtenidas en el análisis del subcomplejo T. sordida (poblaciones agrupadas).

Matriz de estructura

\begin{tabular}{|c|c|c|c|c|c|c|c|}
\hline & \multicolumn{7}{|c|}{ Función } \\
\hline & 1 & 2 & 3 & 4 & 5 & 6 & 7 \\
\hline KI2994 & .344 & $.785^{\star}$ & .021 & .092 & .099 & .435 & .241 \\
\hline KI3175 & .010 & .136 & $.710^{*}$ & .125 & .025 & -.585 & .344 \\
\hline KI2974 & .204 & -.102 & .193 & $.911^{*}$ & -.276 & -.059 & .030 \\
\hline KI3971 & -.608 & .150 & -.025 & .286 & $.680^{*}$ & .119 & -.222 \\
\hline KI3725 & .324 & -.362 & -.375 & -.057 & $.646^{*}$ & -.064 & .445 \\
\hline KI3285 & .099 & -.260 & .609 & -.035 & .247 & $.697^{*}$ & -.054 \\
\hline KI3372 & -.412 & .023 & .210 & -.077 & -.153 & -.165 & $.854^{*}$ \\
\hline
\end{tabular}

Correlaciones intra-grupo combinadas entre las variables discriminantes y las funciones discriminantes canónicas tipificadas

Variables ordenadas por el tamaño de la correlación con la función.

*. May or correlación absoluta entre cada v ariable y cualquier función discriminante. 
Tabla 24. Valores de las funciones discriminantes en los centroides de los grupos del subcomplejo T. sordida (poblaciones agrupadas).

Funciones en los centroides de los grupos

\begin{tabular}{|l|r|r|r|r|r|r|r|}
\hline & \multicolumn{7}{|c|}{ Función } \\
\cline { 2 - 8 } GRUPOS & \multicolumn{1}{|c|}{1} & \multicolumn{1}{|c|}{2} & \multicolumn{1}{c|}{3} & \multicolumn{1}{c|}{4} & \multicolumn{1}{c|}{5} & \multicolumn{1}{c|}{6} & \multicolumn{1}{c|}{7} \\
\hline Tsor Formosa & 3.772 & -4.740 & 2.886 & 2.330 & .309 & 1.452 & .866 \\
Tsor Apolo+Goias & 1.045 & -3.010 & -2.699 & -.691 & -.862 & .335 & 2.065 \\
Tsor Izozog G2 & 3.467 & -2.691 & 4.888 & -1.591 & 1.782 & -2.045 & .458 \\
Tsor Rioverde & 3.182 & -2.993 & -3.093 & -1.307 & .675 & -.338 & -.181 \\
Tsor Rondonopolis & 3.753 & -1.585 & -2.028 & -.491 & .354 & 1.326 & .042 \\
Tsor Tocantins & 3.107 & -2.394 & -2.838 & -1.884 & .759 & -1.096 & -.491 \\
Tsor Boqueron & 1.533 & -2.630 & 3.794 & -1.103 & -1.049 & .611 & -.698 \\
Tsor Con+SPe+Par+Gai & 2.931 & -2.780 & -1.541 & 2.453 & -1.234 & -.670 & -.707 \\
Tsor PteHay es & 2.141 & -2.666 & 2.273 & -1.486 & -.646 & 1.188 & -1.172 \\
Tgar LaRioja & 5.937 & 7.796 & .292 & .786 & .218 & -.027 & .642 \\
Tgar SgoEstero & 5.356 & 6.969 & -.049 & -.477 & -.009 & .345 & -.410 \\
Tgua SgoEstero & -6.384 & 1.191 & .587 & 1.036 & .192 & -1.013 & .024 \\
Tgua Mataral & -6.594 & -.334 & -1.052 & .315 & 3.424 & 1.140 & .498 \\
Tgua Boq+PHay & -8.262 & 1.381 & -.655 & .028 & .948 & 1.073 & -1.494 \\
Tpat SantaFe & -6.787 & 1.160 & .244 & -1.401 & -2.434 & .183 & .587 \\
\hline
\end{tabular}

Funciones discriminantes canónicas no tipificadas evaluadas en las medias de los grupos

Las primeras 3 FD acumulan casi el $90 \%$ de la variabilidad total. La FD 1, influenciada principalmente por los HC con KI 3971 y 3372, contribuye a la separación de las especies $T$. guasayana y $T$. patagonica (valores negativos de la FD). La FD 2, influida principalmente por el HC KI 2994, contribuye a la separación entre T. garciabesi (valores positivos de la FD) y $T$. sordida (valores negativos). La FD 3, influenciada mayormente por el HC KI 3175 contribuye a la separación entre los grupos 1 y 2 de T. sordida (valores negativos para el grupo 1 respecto a valores positivos de la FD para el grupo 2).

En la tabla 25 se computan los resultados de clasificación de los grupos analizados. La diferencia entre los porcentajes de clasificación original y de validación cruzada indica que las funciones discriminantes obtenidas son consistentes para clasificar los especímenes. Los resultados de clasificación parciales muestran que hay tres especies claramente diferenciadas, T. sordida, T. garciabesi y T. guasayana, entre las cuáles no hay errores de clasificación. A partir de la observación de los errores en las clasificaciones parciales, se puede ver que $T$. patagonica muestra un alto grado de superposición respecto a $T$. guasayana. Dentro de $T$. sordida se pueden observar grados de superposición variable entre los especímenes que se corresponden con las subdivisión de la especie en dos grupos (grupo 1 y grupo 2). $T$. garciabesi exhibe una alta superposición entre las dos poblaciones analizadas, producto de su homogeneidad en el perfil de hidrocarburos. 
Tabla 25. Resultados de clasificación de los grupos del subcomplejo T. sordida (poblaciones agrupadas) $(\%)$

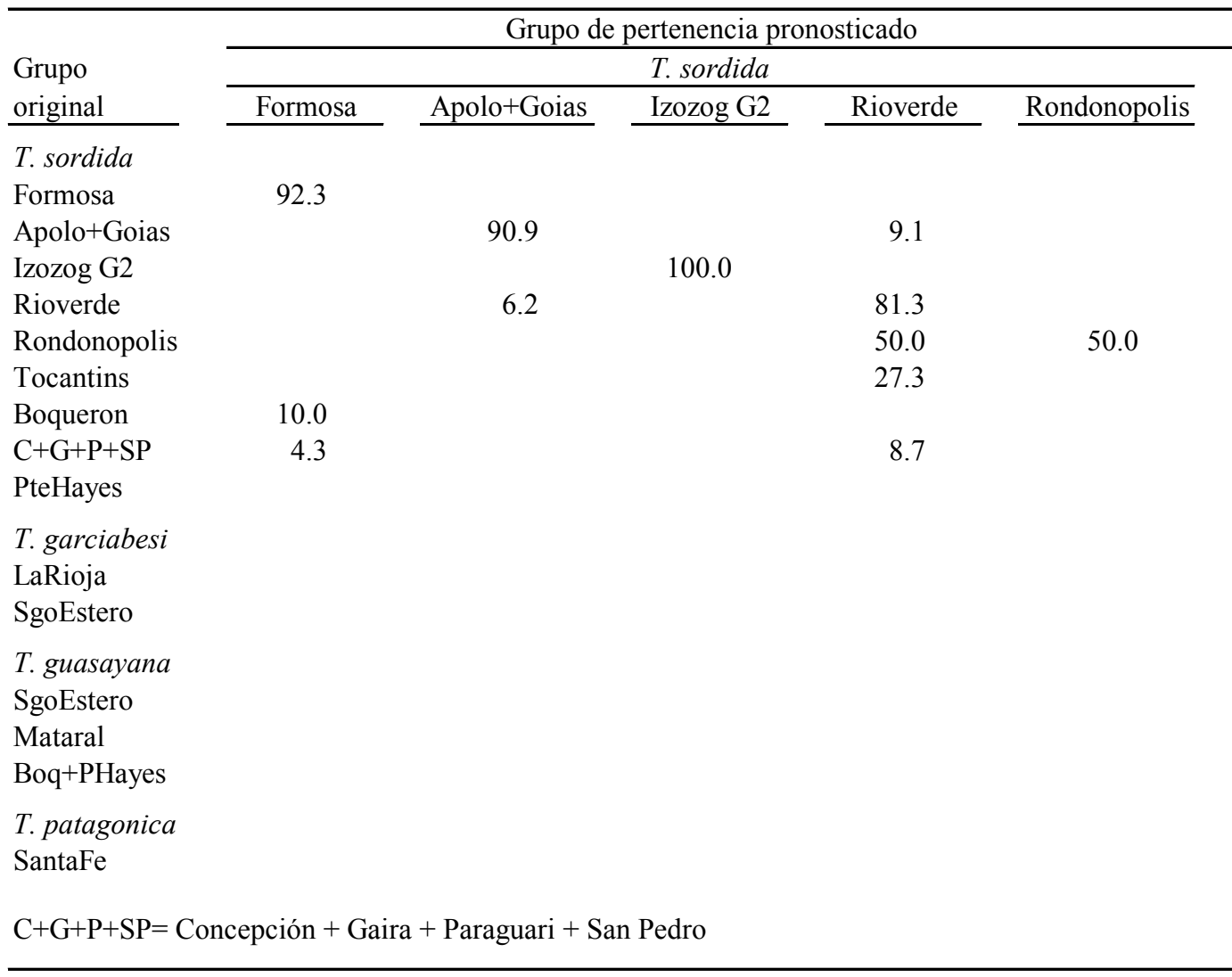

\begin{tabular}{|c|c|c|c|c|}
\hline \multirow{3}{*}{$\begin{array}{l}\text { Grupo } \\
\text { original }\end{array}$} & \multicolumn{4}{|c|}{ Grupo de pertenencia pronosticado } \\
\hline & \multicolumn{4}{|c|}{ T. sordida } \\
\hline & Tocantins & Boqueron & $\mathrm{C}+\mathrm{G}+\mathrm{P}+\mathrm{SP}$ & PteHayes \\
\hline \multicolumn{5}{|l|}{ T. sordida } \\
\hline Formosa & & & & 7.7 \\
\hline \multicolumn{5}{|l|}{ Apolo+Goias } \\
\hline \multicolumn{5}{|l|}{ Izozog G2 } \\
\hline Rioverde & 12.5 & & & \\
\hline \multicolumn{5}{|l|}{ Rondonopolis } \\
\hline Tocantins & 72.7 & & & \\
\hline Boqueron & & 70.0 & & 20.0 \\
\hline $\mathrm{C}+\mathrm{G}+\mathrm{P}+\mathrm{SP}$ & & & 82.7 & 4.3 \\
\hline PteHayes & 9.1 & 27.3 & & 63.6 \\
\hline \multicolumn{5}{|l|}{ T. garciabesi } \\
\hline \multicolumn{5}{|l|}{ LaRioja } \\
\hline \multicolumn{5}{|l|}{ SgoEstero } \\
\hline \multicolumn{5}{|l|}{ T. guasayana } \\
\hline \multicolumn{5}{|l|}{ SgoEstero } \\
\hline \multicolumn{5}{|l|}{ Mataral } \\
\hline \multicolumn{5}{|l|}{ Boq+PHayes } \\
\hline \multicolumn{5}{|l|}{ T. patagonica } \\
\hline \multicolumn{5}{|l|}{ SantaFe } \\
\hline $\mathrm{C}+\mathrm{G}+\mathrm{P}+\mathrm{SP}=$ & epción + Ga & Paraguari & Pedro & \\
\hline
\end{tabular}




\begin{tabular}{|c|c|c|c|c|c|c|}
\hline \multirow{3}{*}{$\begin{array}{l}\text { Grupo } \\
\text { original }\end{array}$} & \multicolumn{6}{|c|}{ Grupo de pertenencia pronosticado } \\
\hline & \multicolumn{2}{|c|}{ T. garciabersi } & \multicolumn{3}{|c|}{ T. guasayana } & \multirow{2}{*}{$\frac{\text { T. patagonica }}{\text { SantaFe }}$} \\
\hline & LaRioja & SgoEstero & SgoEstero & Mataral & Boq+PHayes & \\
\hline \multicolumn{7}{|l|}{ T. sordida } \\
\hline \multicolumn{7}{|l|}{ Formosa } \\
\hline \multicolumn{7}{|l|}{ Apolo+Goias } \\
\hline \multicolumn{7}{|l|}{ Izozog G2 } \\
\hline \multicolumn{7}{|l|}{ Rioverde } \\
\hline \multicolumn{7}{|l|}{ Rondonopolis } \\
\hline \multicolumn{7}{|l|}{ Tocantins } \\
\hline \multicolumn{7}{|l|}{ Boqueron } \\
\hline \multicolumn{7}{|l|}{$\mathrm{C}+\mathrm{G}+\mathrm{P}+\mathrm{SP}$} \\
\hline \multicolumn{7}{|l|}{ PteHayes } \\
\hline \multicolumn{7}{|l|}{ T. garciabesi } \\
\hline LaRioja & 75.0 & 25.0 & & & & \\
\hline SgoEstero & 31.6 & 68.4 & & & & \\
\hline \multicolumn{7}{|l|}{ T. guasayana } \\
\hline SgoEstero & & & 93.8 & & 3.1 & 3.1 \\
\hline Mataral & & & 16.7 & 83.3 & & \\
\hline Boq+PHayes & & & 18.2 & 9.1 & 54.5 & 18.2 \\
\hline \multicolumn{7}{|l|}{ T. patagonica } \\
\hline SantaFe & & & 4.8 & & & 95.2 \\
\hline \multicolumn{7}{|c|}{ Clasificados correctamente el $85.3 \%$ de los casos agrupados originales. } \\
\hline \multicolumn{7}{|c|}{ Clasificados correctamente el $80.9 \%$ de los casos agrupados validados mediante validación cruzada. } \\
\hline $\mathrm{C}+\mathrm{G}+\mathrm{P}+\mathrm{SP}=\mathrm{C}$ & pción $+C$ & + Paraguari & in Pedro & & & \\
\hline
\end{tabular}

Las relaciones entre las cuatro especies del subcomplejo se grafican en un dendrograma basado en la distancia de Mahalanobis entre especies y poblaciones (figura 39). Se pueden reconocer las siguientes características:

- el subcomplejo se estructura en tres agrupaciones principales, una formada por las distintas poblaciones de $T$. sordida, otra por las dos poblaciones analizadas de $T$. garciabesi y la tercera integrada por los ejemplares de $T$. guasayana y $T$. patagonica.

- T. patagonica no se diferencia como especie dentro del subcomplejo quedando incluida dentro de la agrupación formada por las poblaciones de $T$. guasayana.

- T. garciabesi se diferencia como especie, mostrando una relación más estrecha con $T$. sordida.

- T. sordida presenta sus poblaciones separadas en dos grupos claramente diferenciados que se corresponden con los grupos 1 y 2 previamente definidos (pag 76). 
Figura 39. Dendrograma basado en la distancia de Mahalanobis entre las poblaciones agrupadas y especies del subcomplejo T. sordida derivado a partir del DA.

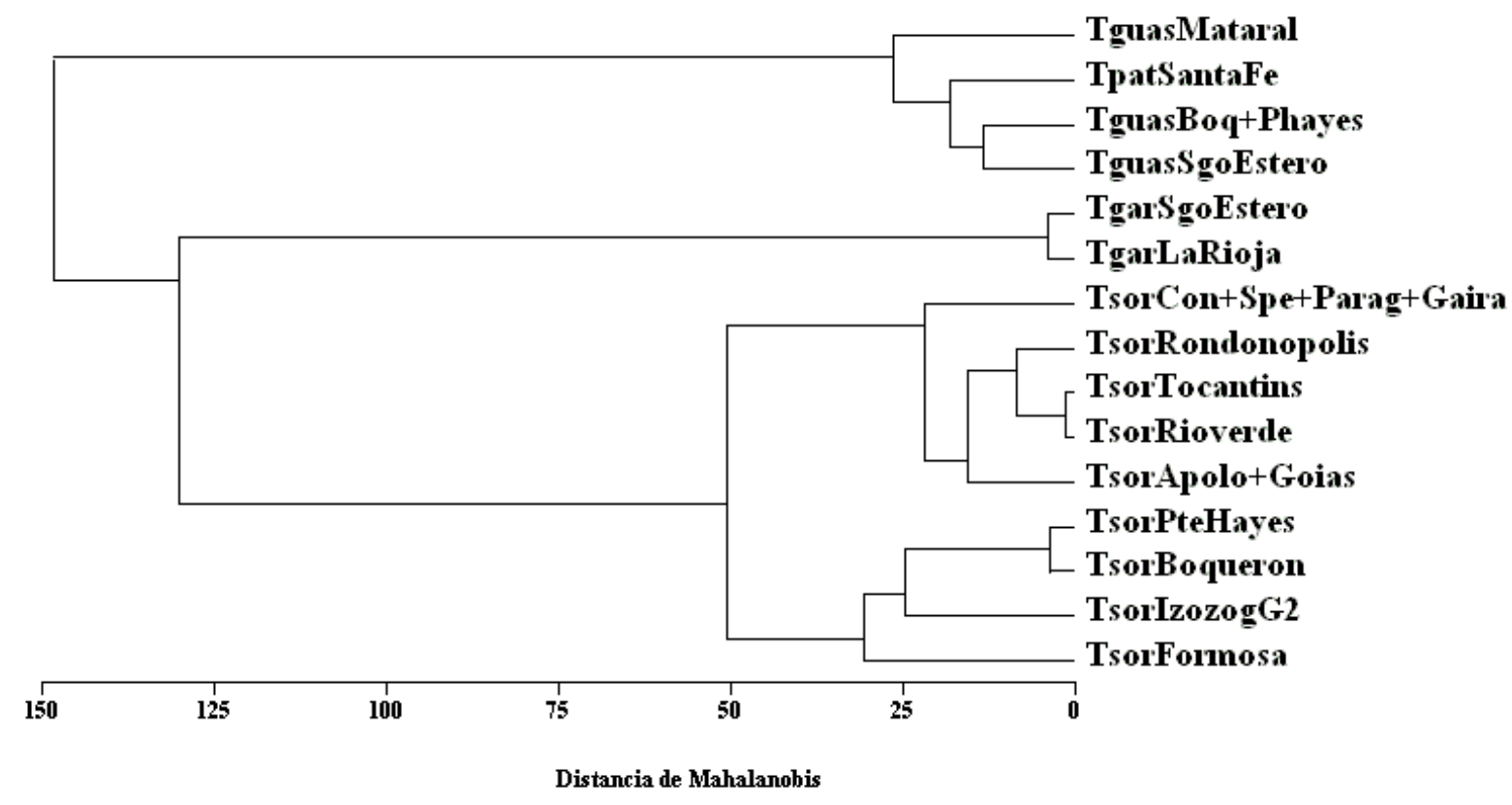

Tras el análisis previo, se analizaron todas las poblaciones de todas las especies del subcomplejo por separado. Para el DA se aplicó el método de eliminación hacia atrás resultando diez HC como variables mejor discriminadoras de todos los grupos estudiados. Los HC seleccionados fueron los de KIs 2858, 2974, 2994, 3175, 3187, 3372, 3378, 3455, 3725 y 3971, con un $\lambda$ de Wilks altamente significativo ( $\mathrm{p} \leq 0.001$ ). Los autovalores, varianzas y correlaciones canónicas de las FD obtenidas se computan en la tabla 26.

Tabla 26. Características de las funciones discriminantes obtenidas en el análisis del subcomplejo T. sordida.

\begin{tabular}{|l|r|r|r|r|}
\hline Autovalores \\
\hline Función & Autov alor & $\%$ de varianza & $\%$ acumulado & $\begin{array}{c}\text { Correlación } \\
\text { canónica }\end{array}$ \\
\hline 1 & $28.546^{\mathrm{a}}$ & 44.4 & 44.4 & .983 \\
2 & $17.056^{\mathrm{a}}$ & 26.5 & 70.9 & .972 \\
3 & $7.542^{\mathrm{a}}$ & 11.7 & 82.6 & .940 \\
4 & $3.418^{\mathrm{a}}$ & 5.3 & 87.9 & .880 \\
5 & $2.571^{\mathrm{a}}$ & 4.0 & 91.9 & .849 \\
6 & $1.889^{\mathrm{a}}$ & 2.9 & 94.8 & .809 \\
7 & $1.108^{\mathrm{a}}$ & 1.7 & 96.6 & .725 \\
8 & $.901^{\mathrm{a}}$ & 1.4 & 98.0 & .689 \\
9 & $.761^{\mathrm{a}}$ & 1.2 & 99.1 & .657 \\
10 & $.549^{\mathrm{a}}$ & .9 & 100.0 & .595 \\
\hline
\end{tabular}


La matriz de estructura y los valores de las FD en los centroides de los grupos se computan en las tablas 27 y 28 respectivamente, mientras que en las figuras 40 a y b se grafican los distintos grupos sobre las tres primeras FD que acumulan un $83 \%$ de la variabilidad total.

Tabla 27. Matriz de estructura de las funciones discriminantes obtenidas en el análisis del subcomplejo T. sordida.

Matriz de estructura

\begin{tabular}{|c|c|c|c|c|c|c|c|c|c|c|}
\hline & \multicolumn{10}{|c|}{ Función } \\
\hline & 1 & 2 & 3 & 4 & 5 & 6 & 7 & 8 & 9 & 10 \\
\hline KI3971 & $-.591^{*}$ & .093 & -.025 & .238 & .235 & .557 & .325 & -.246 & .195 & -.123 \\
\hline $\mathrm{KI} 3725$ & $.370^{*}$ & -.300 & -.368 & .316 & .334 & .331 & .281 & .102 & -.311 & .363 \\
\hline KI2994 & .281 & $.736^{*}$ & .200 & -.203 & -.165 & .398 & .175 & .256 & -.058 & .113 \\
\hline KI3175 & .010 & .095 & $.563^{*}$ & .346 & .388 & -.387 & -.051 & .265 & .324 & .274 \\
\hline KI3187 & .156 & -.098 & $.550^{*}$ & -.108 & -.069 & .149 & .495 & .395 & -.250 & -.401 \\
\hline KI2974 & .201 & -.088 & .205 & .496 & $-.607^{*}$ & -.068 & .108 & .151 & .507 & -.003 \\
\hline $\mathrm{K} 12858$ & .159 & .208 & -.096 & .244 & -.095 & -.433 & $.788^{*}$ & -.111 & .121 & -.137 \\
\hline $\mathrm{KI} 3372$ & -.410 & -.012 & .112 & .018 & .134 & -.285 & .060 & $.604^{*}$ & -.033 & .591 \\
\hline $\mathrm{KI} 3378$ & .161 & -.307 & .403 & -.407 & .006 & .060 & .310 & $-.478^{*}$ & .083 & .465 \\
\hline KI3455 & .146 & -.207 & -.312 & -.424 & .197 & .016 & .404 & .424 & $.525^{*}$ & .033 \\
\hline
\end{tabular}

Correlaciones intra-grupo combinadas entre las variables discriminantes y las funciones discriminantes canónicas tipificadas Variables ordenadas por el tamaño de la correlación con la función.

*. May or correlación absoluta entre cada variable y cualquier función discriminante.

Tabla 28. Valores de las funciones discriminantes en los centroides de los grupos del subcomplejo T. sordida.

Funciones en los centroides de los grupos

\begin{tabular}{|c|c|c|c|c|c|c|c|c|c|c|}
\hline \multirow[b]{2}{*}{ GRUPOS } & \multicolumn{10}{|c|}{ Función } \\
\hline & 1 & 2 & 3 & 4 & 5 & 6 & 7 & 8 & 9 & 10 \\
\hline Tsor Formosa & 3.359 & -5.418 & 4.470 & 1.356 & -2.042 & .646 & 2.121 & .883 & -1.069 & -.276 \\
\hline Tsor Apolo & 1.417 & -2.900 & -3.515 & -2.100 & -.541 & -.689 & 1.142 & 1.679 & .352 & .815 \\
\hline Tsor Izozog G1 & 3.534 & .868 & -5.095 & 1.120 & 1.099 & -4.019 & 3.051 & -1.837 & -1.015 & -2.000 \\
\hline Tsor Izozog G2 & 3. 361 & -3.311 & 4.439 & .824 & 3.868 & -.561 & -.715 & 1.272 & -.125 & -.637 \\
\hline Tsor Quillacollo & 6.206 & -.287 & .362 & 5.649 & 4.715 & -2.384 & .153 & -1.196 & 1.371 & 1. 142 \\
\hline Tsor Goias & 2.246 & -3.000 & -5.570 & -2.515 & 1.559 & -.215 & .740 & 3.786 & 2.458 & .619 \\
\hline Tsor Rioverde & 3.432 & -2.345 & -3.567 & -.421 & .643 & .339 & -.343 & -.553 & -1.052 & .047 \\
\hline Tsor Rondonopolis & 4.162 & -.404 & -3.452 & -1.900 & -.223 & -.153 & 1.883 & -.887 & .766 & .661 \\
\hline Tsor Tocantins & 3.414 & -2.326 & -2.950 & -.736 & 1.481 & .599 & -1.251 & -.424 & -.874 & -183 \\
\hline Tsor Boqueron & 1.569 & -3.977 & 4.420 & -2.926 & .042 & -.517 & -.231 & -.969 & .996 & .619 \\
\hline Tsor Concepcion & 3.047 & -2.396 & -1.534 & 1.408 & -2.275 & -.072 & -.654 & -.128 & .697 & -1.356 \\
\hline Tsor Gaira & 3.671 & -2.050 & -2.650 & 2.750 & -1.527 & -.249 & -.033 & -.540 & -.162 & .466 \\
\hline Tsor Paraguari & 2.375 & -2.465 & -.568 & 1.700 & -2.428 & .393 & -2.137 & -.220 & .662 & .216 \\
\hline Tsor PteHay es & 2.127 & -3.693 & 3.075 & -2.934 & -.214 & .293 & -.543 & -1.508 & .050 & .341 \\
\hline Tsor SanPedro & 2.630 & -2.334 & -.997 & 1.700 & -2.818 & -.168 & -1.086 & -.121 & 1.194 & -.690 \\
\hline Tgar LaRioja & 5.122 & 8.944 & 1.442 & .356 & -.652 & .303 & .387 & -.145 & -.051 & .982 \\
\hline Tgar SgoEstero & 4.762 & 7.625 & .736 & -.995 & .155 & .657 & -.349 & .527 & -.062 & -.917 \\
\hline Tgua SgoEstero & -6.914 & 1.227 & .563 & 1.431 & .106 & -.559 & .252 & -.250 & .503 & .131 \\
\hline TgxTs & 2.296 & -2.248 & -2.036 & 3.105 & -1.578 & .633 & -1.565 & .885 & -.284 & .645 \\
\hline Tgua Mataral & -6.955 & -.513 & -1.163 & 1.215 & 1.294 & 3.439 & .438 & -.108 & -.739 & .651 \\
\hline Tgua Boqueron & -8.909 & .271 & -.878 & -1.848 & .276 & 1.911 & .572 & -.228 & .560 & -.981 \\
\hline Tgua PteHayes & -7.954 & .322 & -.163 & -1.122 & .680 & 1.889 & .523 & -.568 & 1.473 & -1.675 \\
\hline Tpat SantaFe & -7.477 & 1.262 & .138 & -1.336 & -.749 & -2.112 & -.743 & .574 & -1.049 & .185 \\
\hline
\end{tabular}

Funciones discriminantes canónicas no tipificadas ev aluadas en las medias de los grupos 
Figura 40a. Gráfico de puntajes canónicos de las poblaciones y especies del subcomplejo $T$. sordida. Abcisa: FD 2, ordenada: FD 1

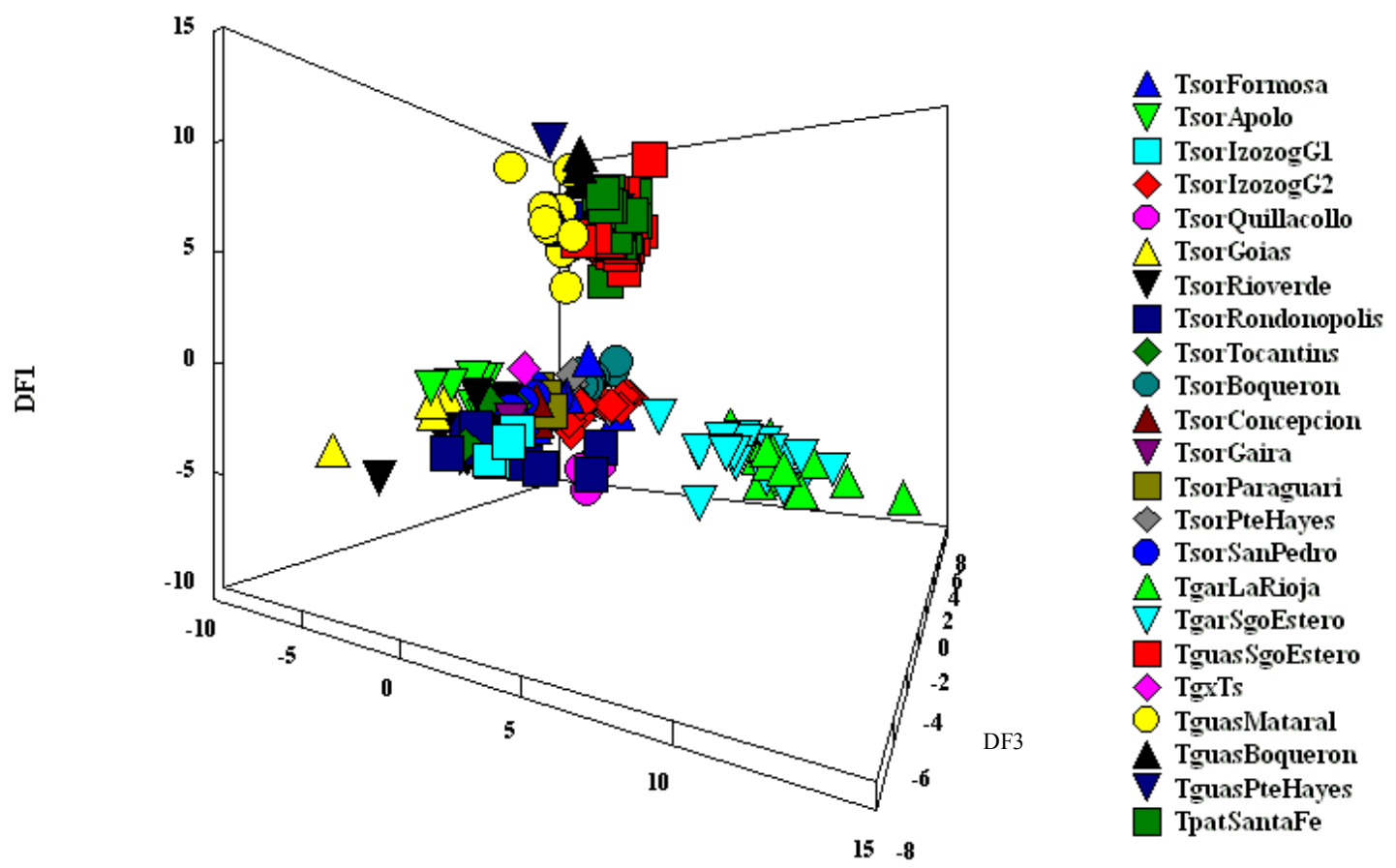

DF2

Figura 40b. Gráfico de puntajes canónicos de las poblaciones y especies del subcomplejo $T$. sordida. Abcisa: FD 1, ordenada: FD 3
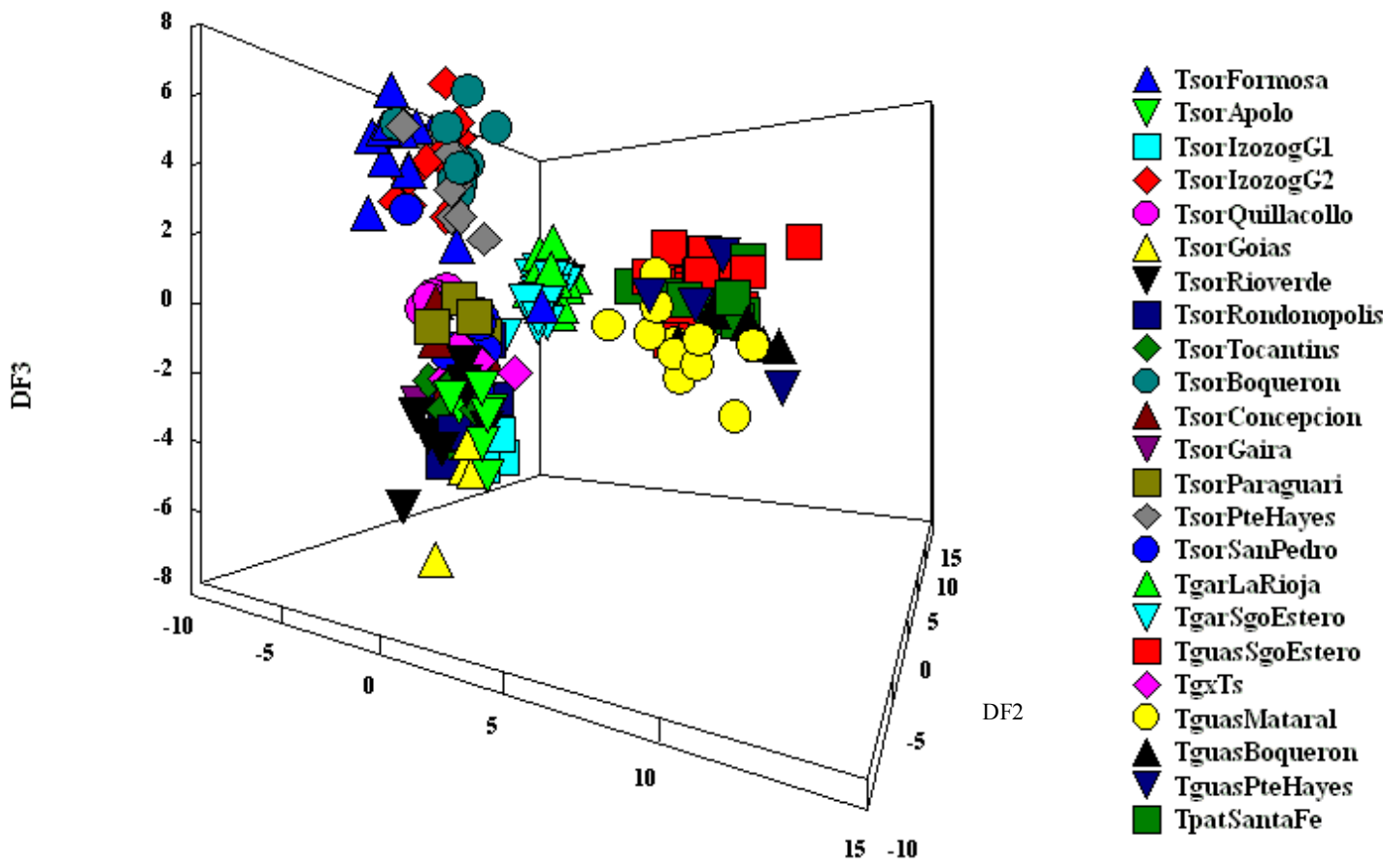
La FD 1, influenciada mayormente por los HC con KI 3971 y 3725 contribuye a la separación de T. guasayana y T. patagonica del resto de los insectos analizados. La FD 2, influenciada principalmente por el HC KI 2994 contribuye a la diferenciación de las dos poblaciones de $T$. garciabesi. La FD 3, influenciada mayormente por los HC con KI 3175 y 3187, contribuye a la diferenciación de los grupos 1 y 2 de la especie $T$. sordida.

El dendrograma basado en las distancias de Mahalanobis entre los centroides de los grupos (figura 41), muestra una serie de características en relación a las cuatro especies estudiadas:

- T. guasayana y T. patagonica son las especies más diferenciadas del complejo, sin embargo, T. patagonica muestra una estrecha relación con los ejemplares de T. guasayana de Santiago del Estero (Argentina), no diferenciándose como especie

- T. garciabesi se diferencia como especie, mostrando una relación más estrecha con $T$. sordida.

- T. sordida muestra una estructuración poblacional muy similar a la obtenida en el análisis de la especie en particular (pag. 77).

- Los insectos designados como TgxTs, quedan agrupados dentro del grupo 1 de T. sordida.

Figura 41. Dendrograma basado en la distancia de Mahalanobis entre las poblaciones agrupadas y especies del subcomplejo T. sordida derivado a partir del DA

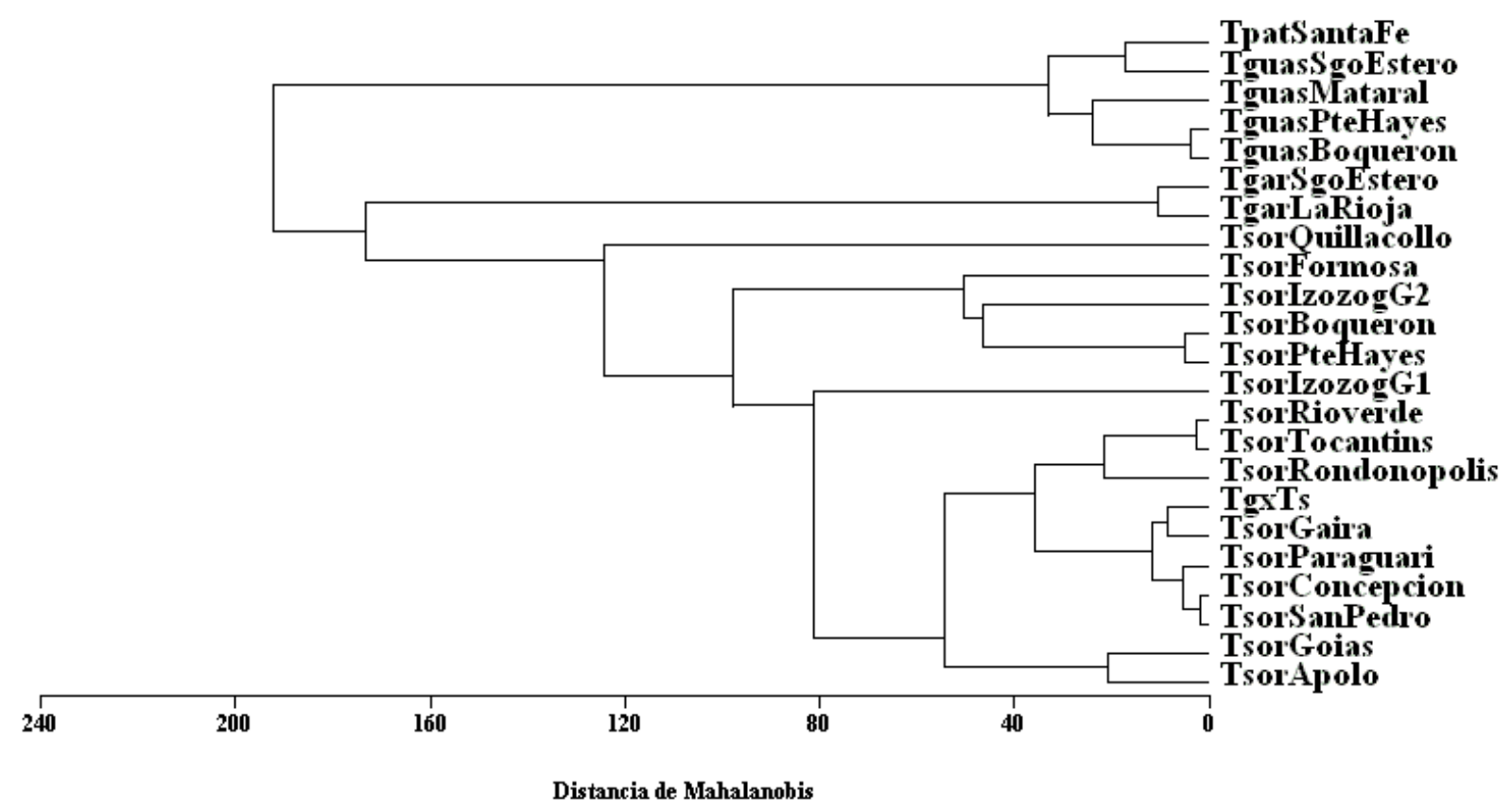


La tabla 29 muestra los resultados de clasificación, donde un $86 \%$ de los especímenes resultaron correctamente clasificados originalmente y un $76 \%$ mediante validación cruzada. Las clasificaciones parciales de los grupos muestran patrones de superposición entre ellos que coinciden con lo mostrado en los gráficos en cuanto al grado de diferenciación de cada especie estudiada.

Tabla 29. Resultados de clasificación de las poblaciones y especies del subcomplejo $T$. sordida $(\%)$.

\begin{tabular}{|c|c|c|c|c|c|}
\hline \multirow{3}{*}{$\begin{array}{l}\text { Grupo } \\
\text { original } \\
\end{array}$} & \multicolumn{5}{|c|}{ Grupo de pertenencia pronosticado } \\
\hline & \multicolumn{5}{|c|}{ T. sordida } \\
\hline & Formosa & Apolo & Izozog G1 & Izozog G2 & Quillacollo \\
\hline \multicolumn{6}{|l|}{ T. sordida } \\
\hline Formosa & 92.3 & & & & \\
\hline Apolo & & 42.9 & & & \\
\hline Izozog G1 & & & 100.0 & & \\
\hline Izozog G2 & & & & 100.0 & \\
\hline Quillacollo & & & & & 100.0 \\
\hline Goias & & 25.0 & & & \\
\hline Rioverde & & 6.2 & & & \\
\hline \multicolumn{6}{|l|}{ Rondonopolis } \\
\hline \multicolumn{6}{|l|}{ Tocantins } \\
\hline Boqueron & 10.0 & & & & \\
\hline \multicolumn{6}{|l|}{ Concepcion } \\
\hline \multicolumn{6}{|l|}{ Gaira } \\
\hline \multicolumn{6}{|l|}{ Paraguari } \\
\hline \multicolumn{6}{|l|}{ PteHayes } \\
\hline SanPedro & 10.0 & & & & \\
\hline \multicolumn{6}{|l|}{ T. garciabesi } \\
\hline \multicolumn{6}{|l|}{ LaRioja } \\
\hline \multicolumn{6}{|l|}{ SgoEstero } \\
\hline \multicolumn{6}{|l|}{ T. guasayana } \\
\hline \multicolumn{6}{|l|}{ SgoEstero } \\
\hline \multicolumn{6}{|l|}{ TgxTs } \\
\hline \multicolumn{6}{|l|}{ Mataral } \\
\hline \multicolumn{6}{|l|}{ Boqueron } \\
\hline \multicolumn{6}{|l|}{ PteHayes } \\
\hline \multicolumn{6}{|l|}{ T. patagonica } \\
\hline SantaFe & & & & & \\
\hline
\end{tabular}

[ LA TABLA CONTINUA EN LA SIGUIENTE HOJA ] 


\begin{tabular}{|c|c|c|c|c|c|}
\hline \multirow{3}{*}{$\begin{array}{l}\text { Grupo } \\
\text { original }\end{array}$} & \multicolumn{5}{|c|}{ Grupo de pertenencia pronosticado } \\
\hline & \multicolumn{5}{|c|}{ T. sordida } \\
\hline & Goias & Rioverde & Rondonopolis & Tocantins & Boqueron \\
\hline \multicolumn{6}{|l|}{ T. sordida } \\
\hline \multicolumn{6}{|l|}{ Formosa } \\
\hline Apolo & 28.6 & 28.5 & & & \\
\hline \multicolumn{6}{|l|}{ Izozog G1 } \\
\hline \multicolumn{6}{|l|}{ Izozog G2 } \\
\hline \multicolumn{6}{|l|}{ Quillacollo } \\
\hline Goias & 75.0 & & & & \\
\hline Rioverde & & 68.8 & & 18.8 & \\
\hline Rondonopolis & & 25.0 & 75.0 & & \\
\hline Tocantins & & 54.5 & & 45.5 & \\
\hline Boqueron & & & & & 50.0 \\
\hline Concepcion & & 40.0 & & & \\
\hline \multicolumn{6}{|l|}{ Gaira } \\
\hline \multicolumn{6}{|l|}{ Paraguari } \\
\hline PteHayes & & & & 9.1 & 36.4 \\
\hline \multicolumn{6}{|l|}{ SanPedro } \\
\hline \multicolumn{6}{|l|}{ T. garciabesi } \\
\hline \multicolumn{6}{|l|}{ LaRioja } \\
\hline \multicolumn{6}{|l|}{ SgoEstero } \\
\hline \multicolumn{6}{|l|}{ T. guasayana } \\
\hline \multicolumn{6}{|l|}{ SgoEstero } \\
\hline \multicolumn{6}{|l|}{ TgxTs } \\
\hline \multicolumn{6}{|l|}{ Mataral } \\
\hline \multicolumn{6}{|l|}{ Boqueron } \\
\hline \multicolumn{6}{|l|}{ PteHayes } \\
\hline \multicolumn{6}{|l|}{ T. patagonica } \\
\hline SantaFe & & & & & \\
\hline
\end{tabular}

\begin{tabular}{|c|c|c|c|c|c|}
\hline \multirow{3}{*}{$\begin{array}{l}\text { Grupo } \\
\text { original }\end{array}$} & \multicolumn{5}{|c|}{ Grupo de pertenencia pronosticado } \\
\hline & \multicolumn{5}{|c|}{ T. sordida } \\
\hline & Concepcion & Gaira & Paraguari & PteHayes & SanPedro \\
\hline \multicolumn{6}{|l|}{ T. sordida } \\
\hline Formosa & & & & 7.7 & \\
\hline \multicolumn{6}{|l|}{ Apolo } \\
\hline \multicolumn{6}{|l|}{ Izozog G1 } \\
\hline \multicolumn{6}{|l|}{ Izozog G2 } \\
\hline \multicolumn{6}{|l|}{ Quillacollo } \\
\hline \multicolumn{6}{|l|}{ Goias } \\
\hline \multicolumn{6}{|l|}{ Rioverde } \\
\hline \multicolumn{6}{|l|}{ Rondonopolis } \\
\hline \multicolumn{6}{|l|}{ Tocantins } \\
\hline Boqueron & & & & 40.0 & \\
\hline Concepcion & & & & & 60.0 \\
\hline Gaira & 33.3 & 33.4 & & & \\
\hline Paraguari & & 20.0 & 40.0 & & 20.0 \\
\hline PteHayes & & & & 54.5 & \\
\hline SanPedro & 20.0 & & & & 70.0 \\
\hline \multicolumn{6}{|l|}{ T. garciabesi } \\
\hline \multicolumn{6}{|l|}{ LaRioja } \\
\hline \multicolumn{6}{|l|}{ SgoEstero } \\
\hline \multicolumn{6}{|l|}{ T. guasayana } \\
\hline \multicolumn{6}{|l|}{ SgoEstero } \\
\hline \multicolumn{6}{|l|}{ TgxTs } \\
\hline \multicolumn{6}{|l|}{ Mataral } \\
\hline \multicolumn{6}{|l|}{ Boqueron } \\
\hline \multicolumn{6}{|l|}{ PteHayes } \\
\hline \multicolumn{6}{|l|}{ T. patagonica } \\
\hline SantaFe & & & & & \\
\hline
\end{tabular}




\begin{tabular}{|c|c|c|c|c|c|}
\hline \multirow{3}{*}{$\begin{array}{l}\text { Grupo } \\
\text { original }\end{array}$} & \multicolumn{5}{|c|}{ Grupo de pertenencia pronosticado } \\
\hline & \multicolumn{2}{|c|}{ T. garciabersi } & \multicolumn{3}{|c|}{ T. guasayana } \\
\hline & LaRioja & SgoEstero & SgoEstero & TgxTs & Mataral \\
\hline \multicolumn{6}{|l|}{ T. sordida } \\
\hline \multicolumn{6}{|l|}{ Formosa } \\
\hline \multicolumn{6}{|l|}{ Apolo } \\
\hline \multicolumn{6}{|l|}{ Izozog G1 } \\
\hline \multicolumn{6}{|l|}{ Izozog G2 } \\
\hline \multicolumn{6}{|l|}{ Quillacollo } \\
\hline \multicolumn{6}{|l|}{ Goias } \\
\hline Rioverde & & & & 6.2 & \\
\hline \multicolumn{6}{|l|}{ Rondonopolis } \\
\hline \multicolumn{6}{|l|}{ Tocantins } \\
\hline \multicolumn{6}{|l|}{ Boqueron } \\
\hline \multicolumn{6}{|l|}{ Concepcion } \\
\hline Gaira & & & & 33.3 & \\
\hline Paraguari & & & & 20.0 & \\
\hline \multicolumn{6}{|l|}{ PteHayes } \\
\hline \multicolumn{6}{|l|}{ SanPedro } \\
\hline \multicolumn{6}{|l|}{ T. garciabesi } \\
\hline LaRioja & 87.5 & 12.5 & & & \\
\hline SgoEstero & 15.8 & 84.2 & & & \\
\hline \multicolumn{6}{|l|}{ T. guasayana } \\
\hline SgoEstero & & & 93.8 & & \\
\hline TgxTs & & & & 100.0 & \\
\hline Mataral & & & & & 91.7 \\
\hline \multicolumn{6}{|l|}{ Boqueron } \\
\hline PteHayes & & & 33.3 & & \\
\hline \multicolumn{6}{|l|}{ T. patagonica } \\
\hline SantaFe & & & 4.8 & & \\
\hline
\end{tabular}

\begin{tabular}{|c|c|c|c|}
\hline \multirow{3}{*}{$\begin{array}{l}\text { Grupo } \\
\text { original }\end{array}$} & \multicolumn{3}{|c|}{ Grupo de pertenencia pronosticado } \\
\hline & \multicolumn{2}{|c|}{ T. guasayana } & \multirow{2}{*}{$\frac{\text { T. patagonica }}{\text { SantaFe }}$} \\
\hline & Boqueron & PteHayes & \\
\hline \\
\hline \multicolumn{4}{|l|}{ Formosa } \\
\hline \multicolumn{4}{|l|}{ Apolo } \\
\hline \multicolumn{4}{|l|}{ Izozog G1 } \\
\hline \multicolumn{4}{|l|}{ Izozog G2 } \\
\hline \multicolumn{4}{|l|}{ Quillacollo } \\
\hline \multicolumn{4}{|l|}{ Goias } \\
\hline \multicolumn{4}{|l|}{ Rioverde } \\
\hline \multicolumn{4}{|l|}{ Rondonopolis } \\
\hline \multicolumn{4}{|l|}{ Tocantins } \\
\hline \multicolumn{4}{|l|}{ Boqueron } \\
\hline \multicolumn{4}{|l|}{ Concepcion } \\
\hline \multicolumn{4}{|l|}{ Gaira } \\
\hline \multicolumn{4}{|l|}{ Paraguari } \\
\hline \multicolumn{4}{|l|}{ PteHayes } \\
\hline \multicolumn{4}{|l|}{ SanPedro } \\
\hline \multicolumn{4}{|l|}{ T. garciabesi } \\
\hline \multicolumn{4}{|l|}{ LaRioja } \\
\hline \multicolumn{4}{|l|}{ SgoEstero } \\
\hline \multicolumn{4}{|l|}{ T. guasayana } \\
\hline SgoEstero & & 3.1 & 3.1 \\
\hline \multicolumn{4}{|l|}{ TgxTs } \\
\hline Mataral & 8.3 & & \\
\hline Boqueron & 40.0 & 60.0 & \\
\hline PteHayes & 33.3 & 33.4 & 18.2 \\
\hline T. patagonica & & & \\
\hline SantaFe & & & 95.2 \\
\hline
\end{tabular}

Clasificados correctamente el $86.2 \%$ de los casos agrupados originales. Clasificados correctamente el $75.7 \%$ de los casos agrupados validados mediante validación cruzada. 


\section{2. Triatoma dimidiata}

Se estudió la composición de los hidrocarburos cuticulares de poblaciones de $T$. dimidiata colectadas en los sitios detallados en el punto III.1, y en base a esta composición, se realizaron los análisis taxonómicos conducentes a establecer las relaciones entre estas poblaciones.

IV. 2. 1. Análisis de los hidrocarburos cuticulares mediante CGC, composición en función de las localidades de colecta.

En las figuras 42, 43, 44, 45, 46 y 47 se muestran los perfiles cromatográficos representativos de las distintas poblaciones analizadas, mientras que en la tabla 30 se computan las cantidades relativas de los hidrocarburos identificados.

Figura 42. Perfiles cromatográficos de las poblaciones de $T$. dimidiata colectadas en Mexico. Tiempo de elución en la abscisa, intensidad de la señal en la ordenada
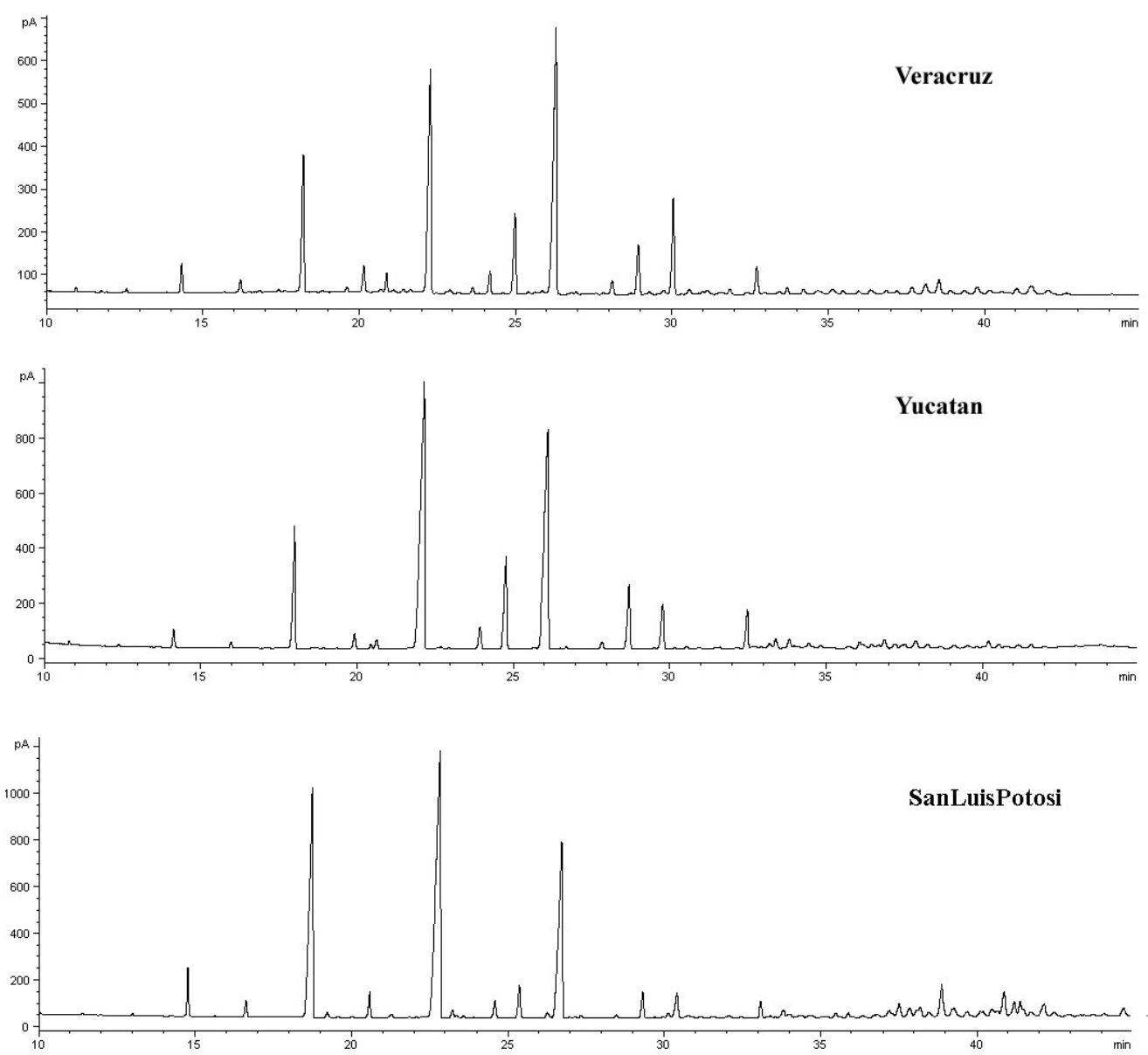

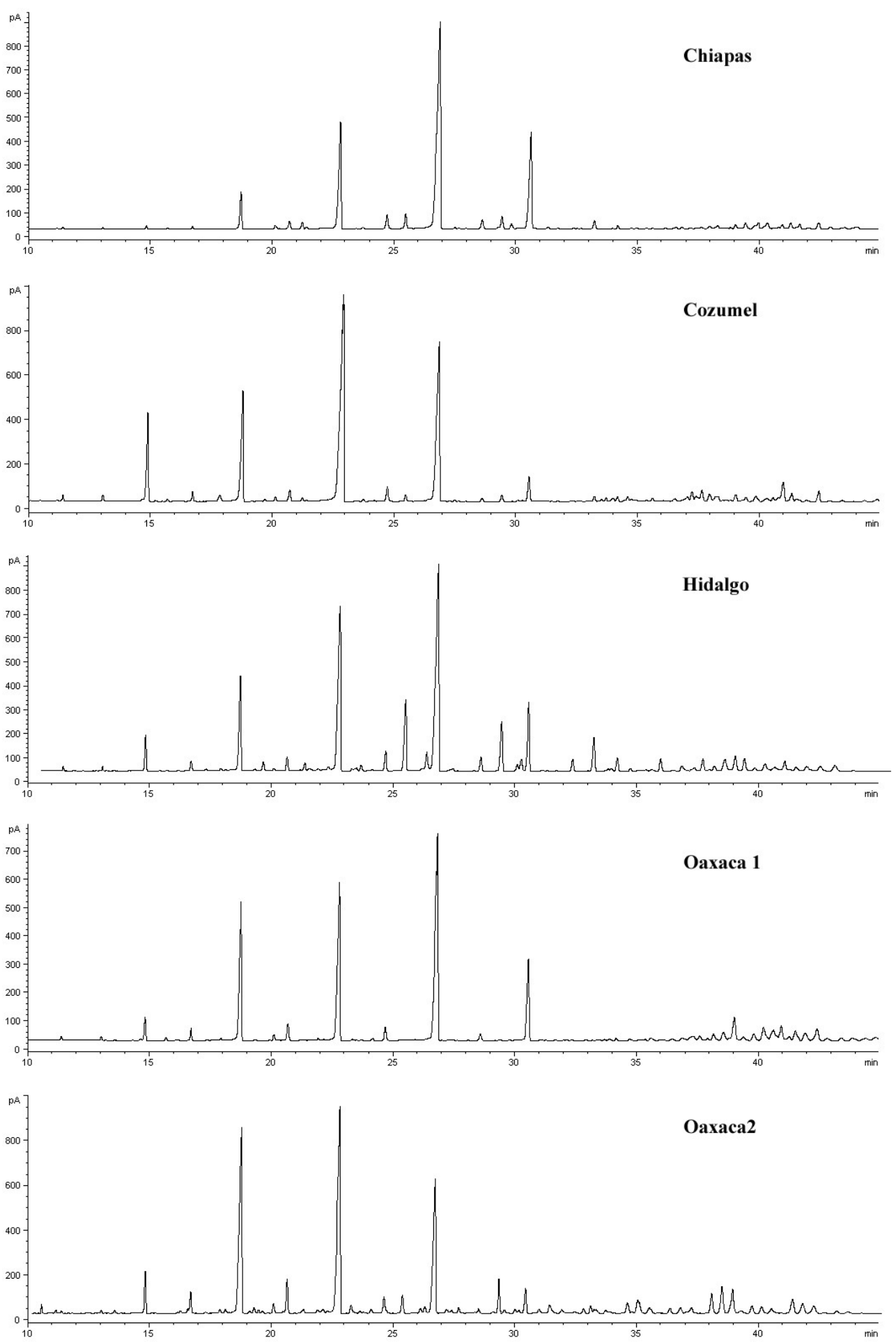

Figura 43. Perfil cromatográfico de la población de $T$. dimidiata colectada en Honduras.

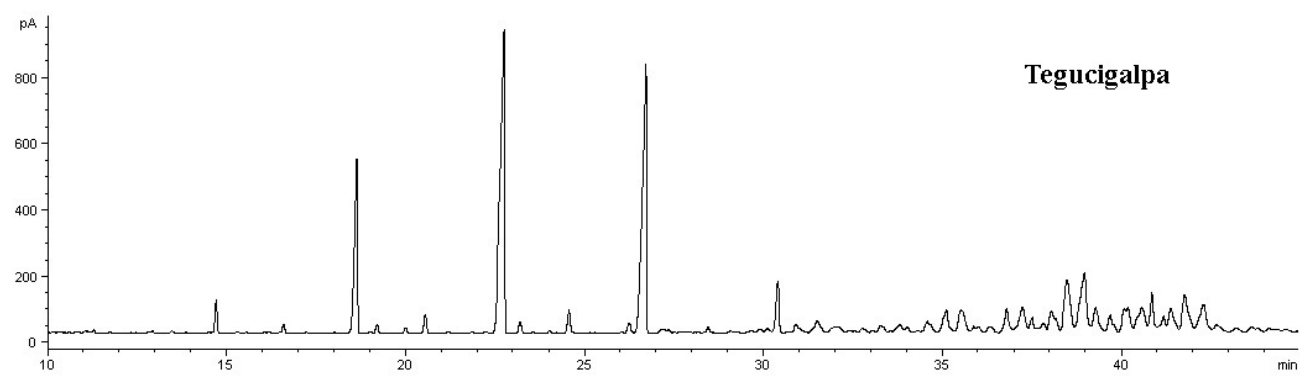


Figura 44. Perfil cromatográfico de la población de $T$. dimidiata colectada en Costa Rica.
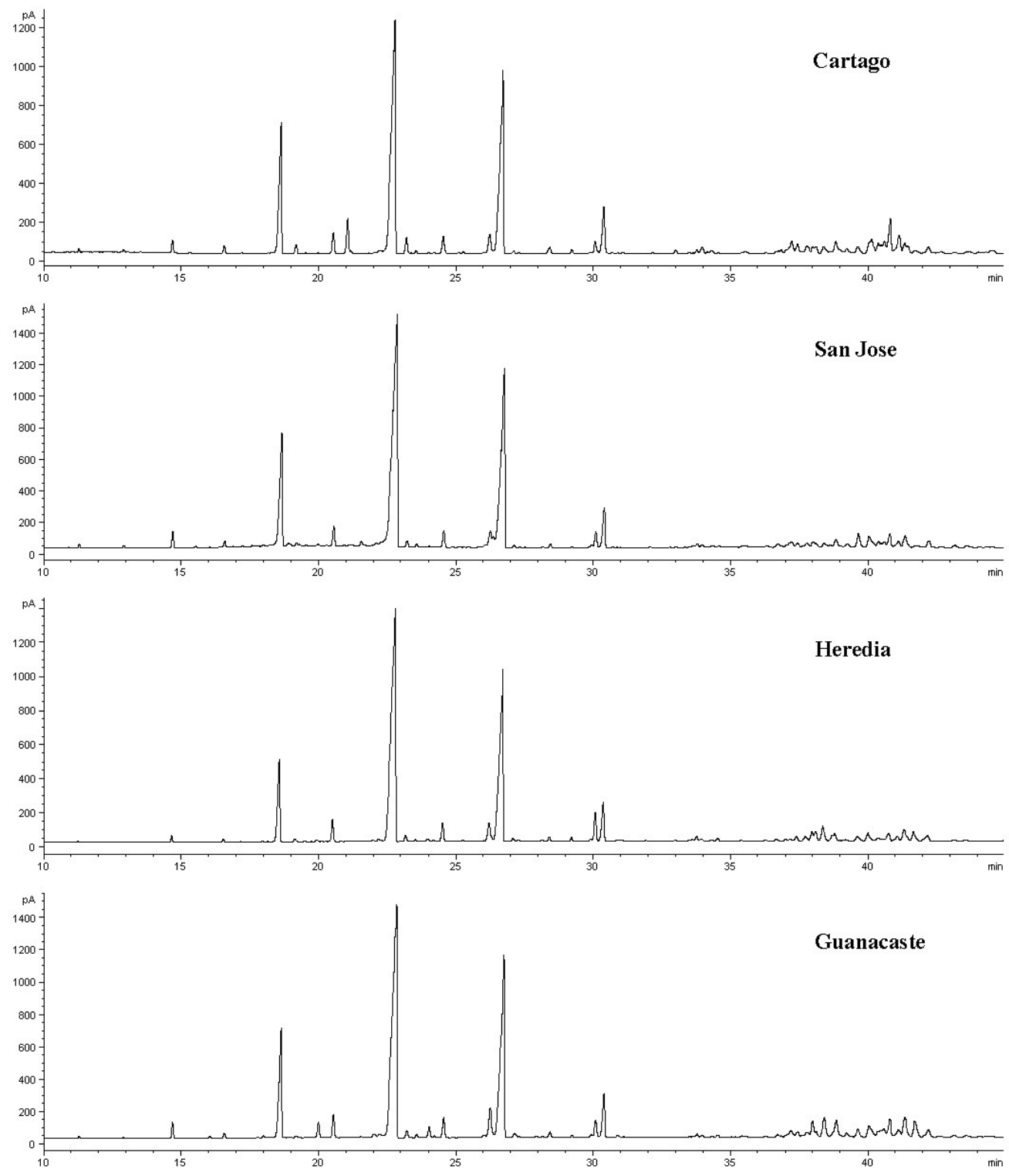

Figura 45. Perfil cromatográfico de la población de $T$. dimidiata colectada en Bélice.

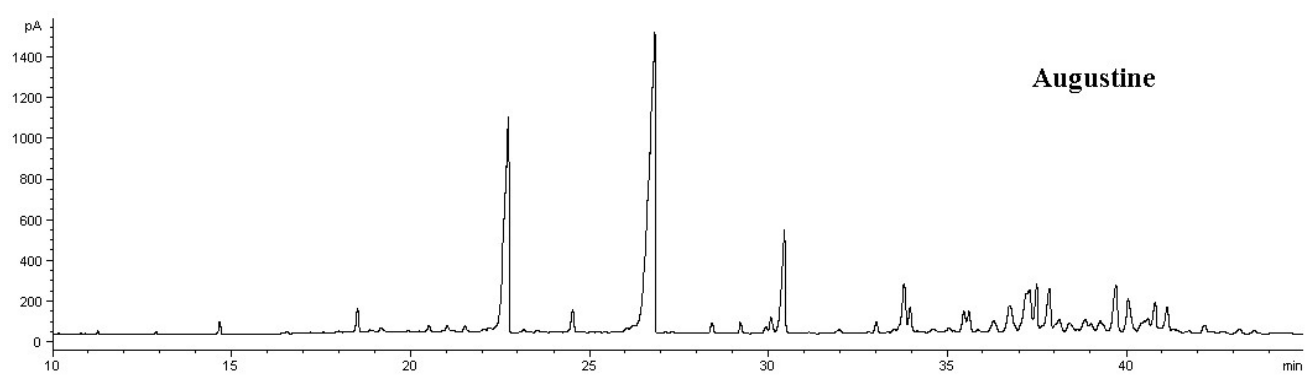


Figura 46. Perfiles cromatográficos de las poblaciones de $T$. dimidiata colectadas en Colombia.
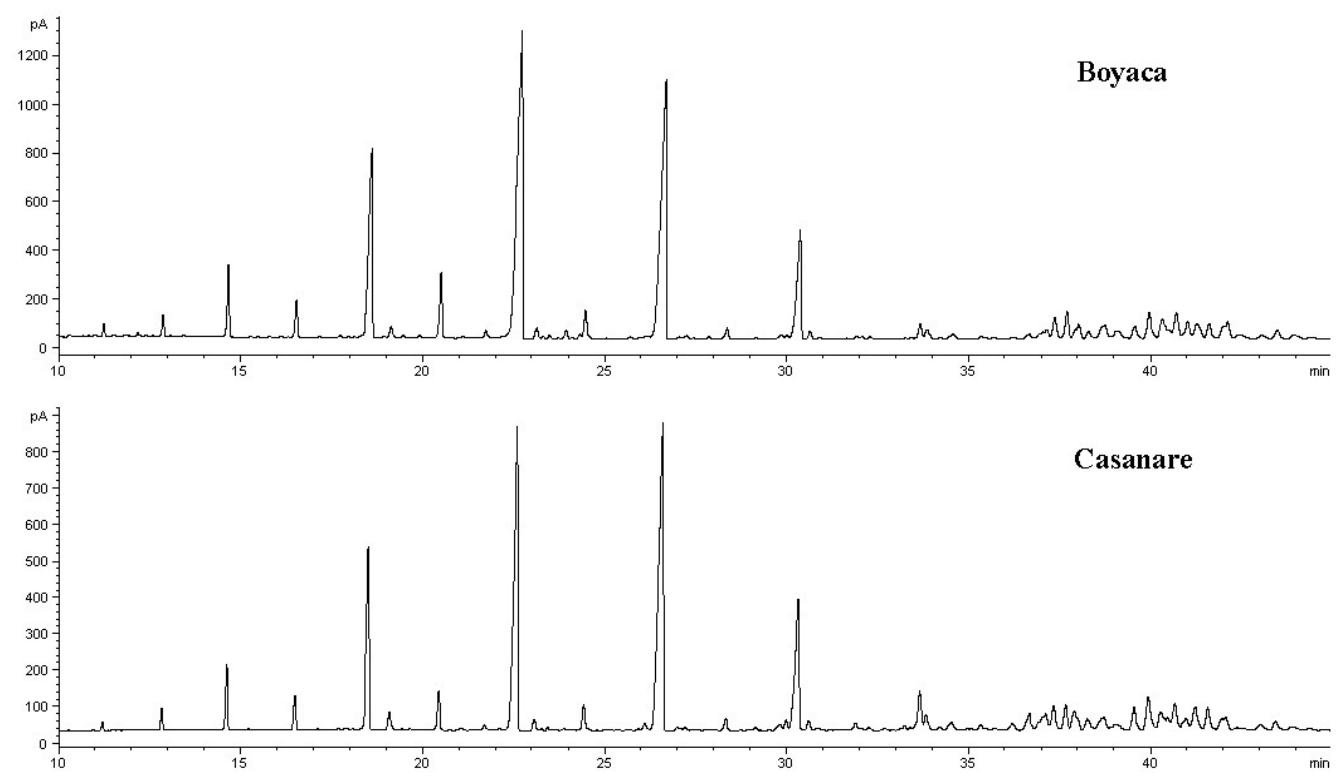

Figura 47. Perfiles cromatográficos de las poblaciones de $T$. dimidiata colectadas en Guatemala.
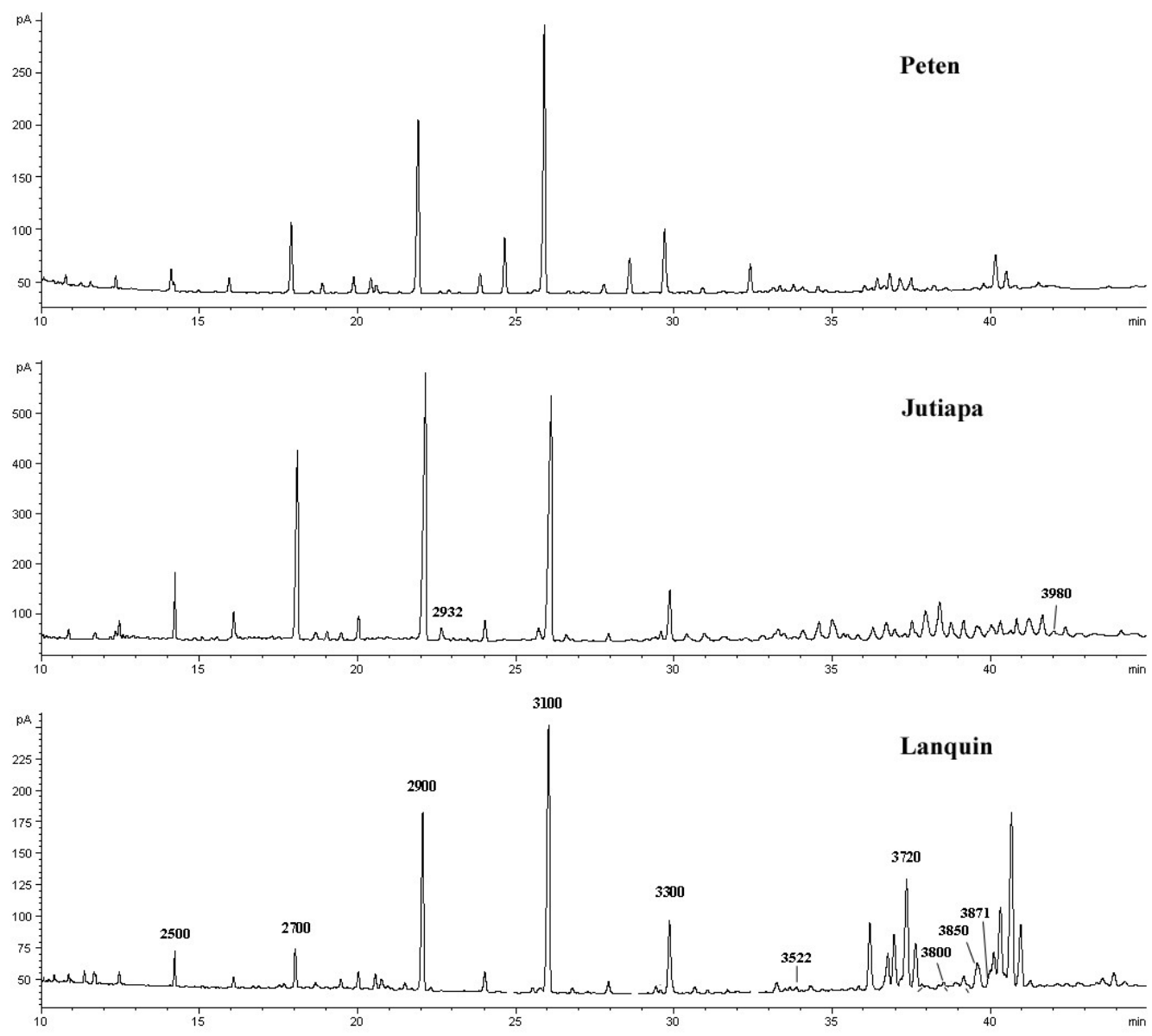

Los números corresponden al KI de los hidrocarburos

Tabla 30. Composición porcentual de los hidrocarburos cuticulares de $T$. dimidiata en relación a las localidades de colecta. [INICIA EN LA SIGUIENTE HOJA] 


\begin{tabular}{|c|c|c|c|c|c|c|c|c|}
\hline \multirow[b]{3}{*}{$\mathrm{KI}$} & \multicolumn{8}{|c|}{ Costa Rica } \\
\hline & \multicolumn{2}{|c|}{ Cartago } & \multicolumn{2}{|c|}{ SanJose } & \multicolumn{2}{|c|}{ Heredia } & \multicolumn{2}{|c|}{ Guanacaste } \\
\hline & media & e.t. & media & e.t. & media & e.t. & media & e.t. \\
\hline 2400 & 0.11 & 0.03 & 0.10 & 0.03 & 0.04 & 0.02 & 0.04 & 0.01 \\
\hline 2500 & 1.24 & 0.32 & 0.83 & 0.11 & 0.86 & 0.20 & 0.54 & 0.10 \\
\hline 2600 & 0.42 & 0.06 & 0.37 & 0.05 & 0.29 & 0.05 & 0.25 & 0.01 \\
\hline 2700 & 7.42 & 1.64 & 8.23 & 1.67 & 8.30 & 1.00 & 8.23 & 0.54 \\
\hline 2800 & 1.12 & 0.08 & 1.23 & 0.12 & 1.50 & 0.14 & 1.54 & 0.13 \\
\hline 2900 & 31.00 & 3.13 & 35.40 & 0.64 & 36.38 & 2.59 & 36.63 & 2.02 \\
\hline 2932 & 0.56 & 0.15 & 0.49 & 0.12 & 0.53 & 0.09 & 0.31 & 0.07 \\
\hline 3000 & 1.06 & 0.12 & 1.28 & 0.10 & 1.27 & 0.05 & 1.50 & 0.12 \\
\hline 3087 & 2.20 & 0.50 & 1.64 & 0.24 & 2.06 & 0.22 & 1.85 & 0.28 \\
\hline 3100 & 21.10 & 2.75 & 26.73 & 1.98 & 22.82 & 1.15 & 24.41 & 1.92 \\
\hline 3200 & 0.33 & 0.10 & 0.35 & 0.04 & 0.28 & 0.03 & 0.41 & 0.02 \\
\hline 3288 & 1.14 & 0.25 & 1.06 & 0.15 & 1.93 & 0.28 & 1.71 & 0.58 \\
\hline 3300 & 2.54 & 0.45 & 3.69 & 0.30 & 3.03 & 0.16 & 3.15 & 0.38 \\
\hline 3477 & 0.23 & 0.09 & 0.09 & 0.03 & 0.11 & 0.04 & 0.16 & 0.07 \\
\hline 3489 & 0.35 & 0.04 & 0.35 & 0.03 & 0.31 & 0.06 & 0.45 & 0.15 \\
\hline 3500 & 0.46 & 0.12 & 0.28 & 0.02 & 0.10 & 0.06 & 0.26 & 0.06 \\
\hline 3522 & 0.30 & 0.08 & 0.22 & 0.05 & 0.03 & 0.03 & 0.06 & 0.04 \\
\hline 3580 & 0.43 & 0.16 & 0.24 & 0.09 & 0.16 & 0.08 & 0.14 & 0.06 \\
\hline 3630 & 0.28 & 0.20 & 0.08 & 0.05 & 0.20 & 0.15 & 0.16 & 0.07 \\
\hline 3655 & 0.76 & 0.31 & 0.31 & 0.08 & 0.55 & 0.23 & 0.34 & 0.11 \\
\hline 3660 & 0.16 & 0.06 & 0.13 & 0.05 & 0.00 & 0.00 & 0.07 & 0.03 \\
\hline 3678 & 0.79 & 0.20 & 0.41 & 0.08 & 0.34 & 0.05 & 0.19 & 0.06 \\
\hline 3686 & 0.43 & 0.28 & 0.34 & 0.20 & 0.00 & 0.00 & 0.36 & 0.16 \\
\hline 3700 & 0.82 & 0.25 & 0.63 & 0.13 & 0.51 & 0.04 & 0.37 & 0.07 \\
\hline 3713 & 0.38 & 0.10 & 0.07 & 0.04 & 0.02 & 0.02 & 0.05 & 0.05 \\
\hline 3720 & 0.67 & 0.25 & 0.71 & 0.14 & 0.51 & 0.05 & 0.34 & 0.07 \\
\hline 3730 & 1.17 & 0.52 & 0.54 & 0.07 & 1.12 & 0.38 & 1.04 & 0.21 \\
\hline 3736 & 0.75 & 0.40 & 0.44 & 0.09 & 0.64 & 0.13 & 0.36 & 0.02 \\
\hline 3755 & 2.11 & 1.11 & 0.99 & 0.22 & 2.55 & 0.93 & 1.86 & 0.61 \\
\hline 3779 & 1.87 & 0.21 & 1.29 & 0.18 & 1.51 & 0.22 & 1.34 & 0.39 \\
\hline 3800 & 0.87 & 0.15 & 0.81 & 0.18 & 0.41 & 0.12 & 0.33 & 0.11 \\
\hline 3826 & 1.22 & 0.17 & 0.99 & 0.18 & 0.66 & 0.06 & 0.60 & 0.12 \\
\hline 3850 & 1.42 & 0.24 & 0.98 & 0.11 & 1.08 & 0.33 & 0.87 & 0.14 \\
\hline 3854 & 0.52 & 0.33 & 0.35 & 0.10 & 0.44 & 0.22 & 0.36 & 0.11 \\
\hline 3871 & 0.64 & 0.13 & 0.38 & 0.05 & 0.44 & 0.08 & 0.52 & 0.08 \\
\hline 3874 & 0.29 & 0.11 & 0.33 & 0.06 & 0.16 & 0.07 & 0.10 & 0.10 \\
\hline 3882 & 0.57 & 0.18 & 0.44 & 0.05 & 0.27 & 0.05 & 0.62 & 0.11 \\
\hline 3894 & 1.54 & 0.60 & 1.19 & 0.13 & 1.11 & 0.10 & 1.27 & 0.24 \\
\hline 3903 & 0.07 & 0.07 & 0.12 & 0.04 & 0.00 & 0.00 & 0.00 & 0.00 \\
\hline 3914 & 1.02 & 0.30 & 0.84 & 0.17 & 0.62 & 0.03 & 0.68 & 0.16 \\
\hline 3926 & 1.00 & 0.35 & 0.87 & 0.12 & 1.10 & 0.15 & 1.10 & 0.35 \\
\hline 3931 & 0.29 & 0.13 & 0.20 & 0.04 & 0.23 & 0.04 & 0.18 & 0.03 \\
\hline 3949 & 0.88 & 0.48 & 0.62 & 0.16 & 1.65 & 0.35 & 1.49 & 0.39 \\
\hline 3980 & 1.34 & 0.21 & 0.87 & 0.04 & 0.75 & 0.11 & 0.59 & 0.10 \\
\hline
\end{tabular}




\begin{tabular}{|c|c|c|c|c|c|c|c|c|}
\hline \multirow[b]{3}{*}{$\mathrm{KI}$} & \multirow{2}{*}{\multicolumn{2}{|c|}{$\frac{\text { Belice }}{\text { Augustine }}$}} & \multicolumn{6}{|c|}{ Guatemala } \\
\hline & & & \multicolumn{2}{|c|}{ Lanquin } & \multicolumn{2}{|c|}{ Peten } & \multicolumn{2}{|c|}{ Jutiapa } \\
\hline & media & e.t. & media & e.t. & media & e.t. & media & e.t. \\
\hline 2400 & 0.14 & 0.02 & 0.54 & 0.06 & 0.59 & 0.14 & 0.36 & 0.05 \\
\hline 2500 & 0.62 & 0.13 & 1.15 & 0.19 & 2.23 & 0.72 & 2.53 & 0.28 \\
\hline 2600 & 0.10 & 0.02 & 0.65 & 0.07 & 1.33 & 0.51 & 1.04 & 0.11 \\
\hline 2700 & 0.90 & 0.17 & 1.53 & 0.19 & 8.14 & 1.62 & 12.13 & 0.72 \\
\hline 2800 & 0.32 & 0.03 & 0.89 & 0.06 & 2.07 & 0.58 & 1.84 & 0.11 \\
\hline 2900 & 16.16 & 0.93 & 14.44 & 2.00 & 22.48 & 1.57 & 33.68 & 1.09 \\
\hline 2932 & 0.12 & 0.02 & 0.00 & 0.00 & 0.00 & 0.00 & 0.38 & 0.05 \\
\hline 3000 & 0.93 & 0.05 & 1.48 & 0.19 & 2.35 & 0.33 & 1.62 & 0.07 \\
\hline 3087 & 0.63 & 0.08 & 0.99 & 0.43 & 0.33 & 0.11 & 0.80 & 0.17 \\
\hline 3100 & 28.40 & 1.93 & 27.86 & 5.40 & 34.86 & 3.47 & 27.39 & 1.04 \\
\hline 3200 & 0.41 & 0.02 & 0.82 & 0.06 & 1.11 & 0.15 & 0.51 & 0.04 \\
\hline 3288 & 0.94 & 0.11 & 0.00 & 0.00 & 0.05 & 0.03 & 0.29 & 0.06 \\
\hline 3300 & 5.20 & 0.24 & 5.70 & 0.54 & 6.80 & 0.73 & 3.77 & 0.26 \\
\hline 3477 & 2.54 & 0.16 & 0.00 & 0.00 & 0.05 & 0.02 & 0.02 & 0.01 \\
\hline 3489 & 0.94 & 0.05 & 0.49 & 0.18 & 0.47 & 0.06 & 0.28 & 0.03 \\
\hline 3500 & 0.09 & 0.03 & 0.00 & 0.00 & 0.63 & 0.07 & 0.29 & 0.03 \\
\hline 3522 & 0.32 & 0.03 & 0.06 & 0.06 & 0.76 & 0.12 & 0.03 & 0.02 \\
\hline 3580 & 0.76 & 0.06 & 0.00 & 0.00 & 0.05 & 0.03 & 0.18 & 0.03 \\
\hline 3630 & 1.98 & 0.32 & 0.05 & 0.05 & 0.00 & 0.00 & 0.11 & 0.03 \\
\hline 3655 & 1.02 & 0.13 & 3.28 & 0.95 & 0.32 & 0.12 & 0.25 & 0.04 \\
\hline 3660 & 2.07 & 0.14 & 0.00 & 0.00 & 0.12 & 0.05 & 0.12 & 0.03 \\
\hline 3678 & 2.35 & 0.28 & 0.33 & 0.15 & 0.77 & 0.14 & 0.14 & 0.03 \\
\hline 3686 & 2.51 & 0.09 & 1.38 & 0.38 & 0.59 & 0.06 & 0.25 & 0.03 \\
\hline 3700 & 0.20 & 0.05 & 2.13 & 0.49 & 1.43 & 0.16 & 0.43 & 0.06 \\
\hline 3713 & 3.05 & 0.12 & 0.30 & 0.18 & 0.05 & 0.05 & 0.00 & 0.00 \\
\hline 3720 & 0.43 & 0.07 & 4.41 & 1.33 & 1.29 & 0.17 & 0.38 & 0.08 \\
\hline 3730 & 0.92 & 0.06 & 0.00 & 0.00 & 0.00 & 0.00 & 0.49 & 0.07 \\
\hline 3736 & 1.00 & 0.11 & 1.92 & 0.56 & 1.30 & 0.19 & 0.37 & 0.08 \\
\hline 3755 & 1.32 & 0.12 & 0.00 & 0.00 & 0.00 & 0.00 & 0.58 & 0.10 \\
\hline 3779 & 0.52 & 0.03 & 0.47 & 0.19 & 0.38 & 0.06 & 0.72 & 0.08 \\
\hline 3800 & 4.07 & 0.26 & 0.24 & 0.24 & 0.03 & 0.03 & 0.25 & 0.04 \\
\hline 3826 & 2.83 & 0.23 & 0.60 & 0.23 & 0.02 & 0.02 & 0.34 & 0.03 \\
\hline 3850 & 0.32 & 0.04 & 1.28 & 0.26 & 0.00 & 0.00 & 0.28 & 0.03 \\
\hline 3854 & 0.58 & 0.06 & 0.54 & 0.20 & 0.00 & 0.00 & 0.19 & 0.03 \\
\hline 3871 & 0.55 & 0.08 & 0.41 & 0.24 & 0.38 & 0.09 & 0.21 & 0.02 \\
\hline 3874 & 0.94 & 0.11 & 0.62 & 0.22 & 0.03 & 0.03 & 0.05 & 0.02 \\
\hline 3882 & 2.10 & 0.13 & 1.77 & 0.30 & 0.17 & 0.04 & 0.17 & 0.02 \\
\hline 3894 & 0.23 & 0.05 & 4.51 & 0.60 & 2.63 & 0.33 & 0.58 & 0.08 \\
\hline 3903 & 1.86 & 0.12 & 0.23 & 0.15 & 0.00 & 0.00 & 0.01 & 0.01 \\
\hline 3914 & 0.28 & 0.06 & 8.39 & 1.78 & 1.64 & 0.17 & 0.39 & 0.06 \\
\hline 3926 & 0.14 & 0.04 & 0.06 & 0.06 & 0.03 & 0.02 & 0.47 & 0.07 \\
\hline 3931 & 0.28 & 0.06 & 3.07 & 0.56 & 0.34 & 0.05 & 0.10 & 0.02 \\
\hline 3949 & 0.93 & 0.11 & 0.25 & 0.15 & 0.00 & 0.00 & 0.27 & 0.05 \\
\hline 3980 & 0.00 & 0.00 & 0.00 & 0.00 & 0.31 & 0.07 & 0.47 & 0.05 \\
\hline
\end{tabular}




\begin{tabular}{|c|c|c|c|c|c|c|c|c|}
\hline \multirow[b]{3}{*}{ KI } & \multirow{2}{*}{\multicolumn{2}{|c|}{$\frac{\text { Honduras }}{\text { Tegucigalpa }}$}} & \multicolumn{4}{|c|}{ Colombia } & \multirow{2}{*}{\multicolumn{2}{|c|}{$\begin{array}{l}\text { Mexico } \\
\text { Hidalgo }\end{array}$}} \\
\hline & & & \multicolumn{2}{|c|}{ Boyaca } & \multicolumn{2}{|c|}{ Casanare } & & \\
\hline & media & e.t. & media & e.t. & media & e.t. & media & e.t. \\
\hline 2400 & 0.01 & 0.01 & 0.12 & 0.01 & 0.05 & 0.01 & 0.34 & 0.04 \\
\hline 2500 & 0.72 & 0.12 & 0.84 & 0.13 & 1.04 & 0.05 & 1.89 & 0.19 \\
\hline 2600 & 0.28 & 0.02 & 0.41 & 0.04 & 0.42 & 0.01 & 0.83 & 0.06 \\
\hline 2700 & 6.78 & 0.62 & 9.22 & 0.88 & 9.57 & 0.46 & 10.78 & 1.05 \\
\hline 2800 & 1.55 & 0.29 & 2.19 & 0.13 & 1.93 & 0.10 & 1.72 & 0.19 \\
\hline 2900 & 27.90 & 2.32 & 30.28 & 1.32 & 29.74 & 1.22 & 21.73 & 1.11 \\
\hline 2932 & 0.08 & 0.05 & 0.22 & 0.06 & 0.32 & 0.05 & 0.31 & 0.07 \\
\hline 3000 & 1.41 & 0.12 & 1.46 & 0.05 & 1.39 & 0.05 & 1.68 & 0.09 \\
\hline 3087 & 0.26 & 0.06 & 0.18 & 0.03 & 0.30 & 0.05 & 0.49 & 0.24 \\
\hline 3100 & 29.57 & 2.64 & 33.69 & 1.62 & 29.18 & 1.22 & 31.72 & 1.84 \\
\hline 3200 & 0.36 & 0.04 & 0.65 & 0.05 & 0.58 & 0.02 & 0.98 & 0.08 \\
\hline 3288 & 0.13 & 0.03 & 0.23 & 0.03 & 0.36 & 0.07 & 0.30 & 0.12 \\
\hline 3300 & 3.68 & 0.31 & 8.52 & 0.79 & 7.24 & 0.41 & 6.72 & 0.61 \\
\hline 3477 & 0.05 & 0.01 & 0.00 & 0.00 & 0.03 & 0.02 & 0.02 & 0.01 \\
\hline 3489 & 0.28 & 0.06 & 0.45 & 0.06 & 1.01 & 0.25 & 0.16 & 0.07 \\
\hline 3500 & 0.25 & 0.02 & 0.44 & 0.07 & 0.60 & 0.12 & 0.55 & 0.09 \\
\hline 3522 & 0.00 & 0.00 & 0.00 & 0.00 & 0.09 & 0.03 & 0.00 & 0.00 \\
\hline 3580 & 0.63 & 0.17 & 0.04 & 0.02 & 0.12 & 0.02 & 0.19 & 0.05 \\
\hline 3630 & 0.21 & 0.04 & 0.02 & 0.02 & 0.11 & 0.05 & 0.13 & 0.05 \\
\hline 3655 & 0.81 & 0.16 & 0.10 & 0.06 & 0.31 & 0.08 & 0.47 & 0.14 \\
\hline 3660 & 0.01 & 0.01 & 0.15 & 0.04 & 0.31 & 0.08 & 0.08 & 0.04 \\
\hline 3678 & 0.18 & 0.04 & 0.17 & 0.02 & 0.31 & 0.04 & 0.12 & 0.03 \\
\hline 3686 & 1.05 & 0.25 & 0.18 & 0.03 & 0.46 & 0.08 & 0.31 & 0.05 \\
\hline 3700 & 0.75 & 0.07 & 0.58 & 0.09 & 0.97 & 0.16 & 0.46 & 0.12 \\
\hline 3713 & 0.02 & 0.02 & 0.00 & 0.00 & 0.00 & 0.00 & 0.01 & 0.01 \\
\hline 3720 & 0.66 & 0.11 & 0.58 & 0.09 & 0.99 & 0.13 & 0.13 & 0.05 \\
\hline 3730 & 0.70 & 0.15 & 0.30 & 0.06 & 0.43 & 0.08 & 0.53 & 0.07 \\
\hline 3736 & 0.72 & 0.11 & 0.33 & 0.07 & 0.49 & 0.06 & 0.03 & 0.02 \\
\hline 3755 & 1.96 & 0.63 & 0.26 & 0.04 & 0.40 & 0.06 & 1.08 & 0.13 \\
\hline 3779 & 0.99 & 0.23 & 0.67 & 0.09 & 0.83 & 0.13 & 1.74 & 0.16 \\
\hline 3800 & 1.08 & 0.20 & 0.38 & 0.07 & 0.45 & 0.06 & 0.71 & 0.11 \\
\hline 3826 & 0.80 & 0.09 & 0.54 & 0.05 & 0.69 & 0.06 & 0.63 & 0.11 \\
\hline 3850 & 0.75 & 0.12 & 0.87 & 0.09 & 1.14 & 0.11 & 1.02 & 0.17 \\
\hline 3854 & 1.05 & 0.13 & 0.15 & 0.05 & 0.20 & 0.06 & 0.08 & 0.03 \\
\hline 3871 & 0.51 & 0.07 & 0.65 & 0.07 & 0.79 & 0.09 & 0.07 & 0.04 \\
\hline 3874 & 1.07 & 0.26 & 0.11 & 0.05 & 0.20 & 0.06 & 0.60 & 0.08 \\
\hline 3882 & 0.25 & 0.10 & 0.19 & 0.01 & 0.37 & 0.05 & 0.09 & 0.04 \\
\hline 3894 & 1.98 & 0.16 & 0.83 & 0.09 & 1.10 & 0.15 & 0.36 & 0.18 \\
\hline 3903 & 0.08 & 0.04 & 0.02 & 0.02 & 0.01 & 0.01 & 0.37 & 0.12 \\
\hline 3914 & 1.12 & 0.12 & 0.42 & 0.06 & 0.53 & 0.07 & 0.19 & 0.06 \\
\hline 3926 & 1.19 & 0.19 & 0.67 & 0.04 & 0.95 & 0.09 & 0.64 & 0.10 \\
\hline 3931 & 0.35 & 0.05 & 0.15 & 0.04 & 0.13 & 0.04 & 0.06 & 0.04 \\
\hline 3949 & 1.43 & 0.43 & 0.57 & 0.08 & 0.66 & 0.11 & 0.72 & 0.12 \\
\hline 3980 & 1.78 & 0.41 & 0.38 & 0.05 & 0.44 & 0.08 & 0.76 & 0.12 \\
\hline
\end{tabular}




\begin{tabular}{|c|c|c|c|c|c|c|c|c|}
\hline \multirow[b]{3}{*}{$\mathrm{KI}$} & \multicolumn{8}{|c|}{ Mexico } \\
\hline & \multicolumn{2}{|c|}{ Veracruz } & \multicolumn{2}{|c|}{ Yucatan } & \multicolumn{2}{|c|}{ Cozumel } & \multicolumn{2}{|c|}{ Oaxaca1 } \\
\hline & media & e.t. & media & e.t. & media & e.t. & media & e.t. \\
\hline 2400 & 0.19 & 0.02 & 0.24 & 0.04 & 0.36 & 0.07 & 0.21 & 0.05 \\
\hline 2500 & 1.68 & 0.21 & 1.78 & 0.26 & 3.25 & 0.79 & 1.36 & 0.14 \\
\hline 2600 & 0.67 & 0.06 & 0.60 & 0.04 & 0.66 & 0.05 & 0.84 & 0.04 \\
\hline 2700 & 8.29 & 0.66 & 9.24 & 0.79 & 9.88 & 1.04 & 9.50 & 1.90 \\
\hline 2800 & 1.25 & 0.14 & 1.19 & 0.09 & 0.99 & 0.09 & 1.23 & 0.12 \\
\hline 2900 & 17.11 & 1.83 & 27.15 & 1.30 & 27.32 & 2.09 & 16.30 & 1.19 \\
\hline 2932 & 0.53 & 0.13 & 0.05 & 0.02 & 0.04 & 0.02 & 0.26 & 0.21 \\
\hline 3000 & 1.61 & 0.07 & 1.96 & 0.13 & 1.54 & 0.15 & 1.44 & 0.13 \\
\hline 3087 & 0.42 & 0.14 & 0.19 & 0.05 & 0.33 & 0.09 & 0.00 & 0.00 \\
\hline 3100 & 31.42 & 1.90 & 31.16 & 1.37 & 26.02 & 2.06 & 34.50 & 3.39 \\
\hline 3200 & 0.99 & 0.05 & 0.90 & 0.06 & 0.60 & 0.13 & 0.83 & 0.12 \\
\hline 3288 & 0.27 & 0.09 & 0.09 & 0.03 & 0.21 & 0.08 & 0.00 & 0.00 \\
\hline 3300 & 6.93 & 0.55 & 5.25 & 0.39 & 4.28 & 0.68 & 10.00 & 2.11 \\
\hline 3477 & 0.02 & 0.01 & 0.09 & 0.02 & 0.30 & 0.06 & 0.05 & 0.03 \\
\hline 3489 & 0.28 & 0.07 & 0.51 & 0.06 & 0.61 & 0.09 & 0.16 & 0.04 \\
\hline 3500 & 0.42 & 0.06 & 0.85 & 0.06 & 0.77 & 0.11 & 0.35 & 0.12 \\
\hline 3522 & 0.09 & 0.09 & 0.71 & 0.06 & 0.72 & 0.17 & 0.00 & 0.00 \\
\hline 3580 & 0.53 & 0.09 & 0.25 & 0.04 & 0.25 & 0.04 & 0.53 & 0.27 \\
\hline 3630 & 0.28 & 0.08 & 0.36 & 0.06 & 0.46 & 0.06 & 0.25 & 0.16 \\
\hline 3655 & 0.40 & 0.09 & 1.33 & 0.24 & 0.47 & 0.12 & 0.48 & 0.28 \\
\hline 3660 & 0.26 & 0.09 & 0.28 & 0.07 & 0.53 & 0.08 & 0.04 & 0.04 \\
\hline 3678 & 0.12 & 0.06 & 0.57 & 0.05 & 1.23 & 0.17 & 0.13 & 0.07 \\
\hline 3686 & 0.73 & 0.12 & 0.53 & 0.07 & 0.94 & 0.10 & 0.67 & 0.15 \\
\hline 3700 & 0.54 & 0.12 & 1.07 & 0.08 & 1.75 & 0.30 & 0.40 & 0.13 \\
\hline 3713 & 0.03 & 0.03 & 0.00 & 0.00 & 0.28 & 0.15 & 0.02 & 0.02 \\
\hline 3720 & 0.25 & 0.12 & 0.57 & 0.07 & 0.86 & 0.30 & 0.24 & 0.09 \\
\hline 3730 & 0.68 & 0.11 & 0.45 & 0.05 & 0.50 & 0.05 & 0.77 & 0.14 \\
\hline 3736 & 0.08 & 0.05 & 0.28 & 0.07 & 0.69 & 0.19 & 0.06 & 0.06 \\
\hline 3755 & 1.54 & 0.22 & 0.93 & 0.17 & 0.79 & 0.24 & 1.42 & 0.46 \\
\hline 3779 & 2.82 & 0.41 & 0.68 & 0.07 & 0.72 & 0.10 & 2.74 & 0.74 \\
\hline 3800 & 0.91 & 0.10 & 0.46 & 0.05 & 0.61 & 0.11 & 0.58 & 0.07 \\
\hline 3826 & 0.78 & 0.10 & 0.69 & 0.08 & 0.74 & 0.10 & 0.93 & 0.05 \\
\hline 3850 & 1.45 & 0.20 & 0.53 & 0.08 & 0.42 & 0.10 & 1.78 & 0.26 \\
\hline 3854 & 0.15 & 0.09 & 0.15 & 0.03 & 0.53 & 0.13 & 0.12 & 0.08 \\
\hline 3871 & 0.17 & 0.12 & 0.32 & 0.06 & 0.63 & 0.14 & 0.18 & 0.18 \\
\hline 3874 & 1.06 & 0.19 & 0.00 & 0.00 & 0.06 & 0.04 & 1.30 & 0.36 \\
\hline 3882 & 0.18 & 0.09 & 0.28 & 0.05 & 0.52 & 0.06 & 0.17 & 0.09 \\
\hline 3894 & 0.86 & 0.39 & 1.15 & 0.15 & 2.89 & 0.59 & 1.06 & 0.48 \\
\hline 3903 & 0.31 & 0.10 & 0.00 & 0.00 & 0.02 & 0.02 & 0.05 & 0.05 \\
\hline 3914 & 0.42 & 0.17 & 0.60 & 0.11 & 1.45 & 0.42 & 0.44 & 0.14 \\
\hline 3926 & 0.76 & 0.05 & 0.29 & 0.04 & 0.30 & 0.05 & 1.03 & 0.22 \\
\hline 3931 & 0.15 & 0.08 & 0.08 & 0.03 & 0.27 & 0.09 & 0.10 & 0.10 \\
\hline 3949 & 1.33 & 0.13 & 0.64 & 0.15 & 0.42 & 0.14 & 1.39 & 0.60 \\
\hline 3980 & 1.29 & 0.20 & 0.62 & 0.09 & 0.96 & 0.20 & 1.72 & 0.50 \\
\hline
\end{tabular}




\begin{tabular}{|c|c|c|c|c|c|c|}
\hline \multirow[b]{3}{*}{$\mathrm{KI}$} & \multicolumn{6}{|c|}{ Mexico } \\
\hline & \multicolumn{2}{|c|}{ Chiapas } & \multicolumn{2}{|c|}{ Oaxaca2 } & \multicolumn{2}{|c|}{ SLPotosi } \\
\hline & media & e.t. & media & e.t. & media & e.t. \\
\hline 2400 & 0.18 & 0.02 & 0.16 & 0.03 & 0.18 & 0.05 \\
\hline 2500 & 0.59 & 0.13 & 2.46 & 0.12 & 1.83 & 0.14 \\
\hline 2600 & 0.46 & 0.07 & 1.13 & 0.08 & 0.69 & 0.08 \\
\hline 2700 & 7.11 & 1.24 & 18.30 & 1.00 & 9.76 & 1.44 \\
\hline 2800 & 1.23 & 0.22 & 1.99 & 0.11 & 1.09 & 0.16 \\
\hline 2900 & 17.56 & 0.99 & 27.45 & 1.25 & 15.61 & 1.30 \\
\hline 2932 & 0.03 & 0.02 & 0.65 & 0.09 & 0.30 & 0.06 \\
\hline 3000 & 1.73 & 0.06 & 0.87 & 0.11 & 1.46 & 0.13 \\
\hline 3087 & 0.17 & 0.07 & 0.46 & 0.16 & 0.53 & 0.14 \\
\hline 3100 & 44.27 & 2.06 & 11.33 & 0.91 & 29.51 & 2.23 \\
\hline 3200 & 1.13 & 0.05 & 0.22 & 0.05 & 0.75 & 0.03 \\
\hline 3288 & 0.10 & 0.04 & 0.22 & 0.09 & 0.30 & 0.08 \\
\hline 3300 & 13.28 & 1.10 & 1.43 & 0.13 & 6.56 & 0.58 \\
\hline 3477 & 0.00 & 0.00 & 0.18 & 0.08 & 0.06 & 0.03 \\
\hline 3489 & 0.06 & 0.04 & 0.11 & 0.04 & 0.48 & 0.07 \\
\hline 3500 & 0.37 & 0.04 & 0.07 & 0.06 & 0.18 & 0.04 \\
\hline 3522 & 0.00 & 0.00 & 0.11 & 0.04 & 0.13 & 0.06 \\
\hline 3580 & 0.10 & 0.02 & 0.68 & 0.19 & 0.94 & 0.26 \\
\hline 3630 & 0.09 & 0.04 & 0.38 & 0.08 & 0.45 & 0.10 \\
\hline 3655 & 0.13 & 0.06 & 0.61 & 0.06 & 0.70 & 0.12 \\
\hline 3660 & 0.00 & 0.00 & 0.12 & 0.05 & 0.03 & 0.03 \\
\hline 3678 & 0.04 & 0.02 & 0.84 & 0.08 & 1.03 & 0.16 \\
\hline 3686 & 0.10 & 0.05 & 0.31 & 0.12 & 0.03 & 0.03 \\
\hline 3700 & 0.28 & 0.05 & 0.00 & 0.00 & 0.44 & 0.15 \\
\hline 3713 & 0.00 & 0.00 & 0.49 & 0.16 & 0.00 & 0.00 \\
\hline 3720 & 0.45 & 0.07 & 0.00 & 0.00 & 0.51 & 0.11 \\
\hline 3730 & 0.29 & 0.09 & 1.56 & 0.30 & 0.91 & 0.16 \\
\hline 3736 & 0.47 & 0.07 & 0.43 & 0.20 & 0.18 & 0.09 \\
\hline 3755 & 0.20 & 0.09 & 4.69 & 0.44 & 2.34 & 0.44 \\
\hline 3779 & 0.83 & 0.09 & 4.45 & 0.52 & 3.35 & 0.65 \\
\hline 3800 & 0.54 & 0.07 & 0.57 & 0.08 & 0.64 & 0.15 \\
\hline 3826 & 0.57 & 0.06 & 0.62 & 0.07 & 0.97 & 0.20 \\
\hline 3850 & 0.39 & 0.05 & 1.51 & 0.19 & 2.10 & 0.45 \\
\hline 3854 & 0.92 & 0.22 & 0.00 & 0.00 & 0.04 & 0.04 \\
\hline 3871 & 0.00 & 0.00 & 0.00 & 0.00 & 0.03 & 0.03 \\
\hline 3874 & 0.44 & 0.07 & 1.16 & 0.18 & 1.57 & 0.32 \\
\hline 3882 & 0.24 & 0.04 & 0.03 & 0.03 & 0.18 & 0.08 \\
\hline 3894 & 0.84 & 0.17 & 0.34 & 0.14 & 1.32 & 0.36 \\
\hline 3903 & 0.00 & 0.00 & 0.00 & 0.00 & 0.00 & 0.00 \\
\hline 3914 & 0.86 & 0.16 & 0.34 & 0.13 & 0.68 & 0.19 \\
\hline 3926 & 0.34 & 0.08 & 0.92 & 0.19 & 0.72 & 0.13 \\
\hline 3931 & 0.67 & 0.09 & 0.22 & 0.10 & 0.37 & 0.16 \\
\hline 3949 & 0.26 & 0.07 & 1.86 & 0.22 & 2.10 & 0.41 \\
\hline 3980 & 1.16 & 0.04 & 1.83 & 0.18 & 2.08 & 0.38 \\
\hline
\end{tabular}

et: error estándar o típico de la media 
Los componentes mayoritarios son los HC lineales de 27 (KI 2700), 29 (KI 2900), 31 (KI 3100) y 33 (KI 3300) carbonos, que varían entre el 50 al $90 \%$ de la fracción total.

Dentro de los HC lineales, el $n$-C31 es el mayor componente en los especímenes de Bélice, Lanquín, Petén, Hidalgo, Oaxaca 1, Veracruz y Chiapas, llegando en esta última población a cerca del $40 \%$ de los HC totales. El $n$-C29 es el componente mayoritario en los insectos de Costa Rica, Jutiapa y Santa Rosa; mientras que en los de Cozumél, Yucatán, Colombia y Honduras su proporción es similar al $n$-C31. El $n$-C27 varía en la mayoria de las poblaciones en rangos del 6.3 al $10.4 \%$, excepto los insectos de Oaxaca 2 que llega al $19 \%$, mientras que lo insectos de Lanquín y Bélice exhiben valores cercanos al $1 \%$. El n-C33 alcanza altas proporciones en los insectos de Colombia, Oaxaca 1 y Chiapas, alcanzando valores que oscilan entre el 7 al $13 \%$ del total de HC; valores intermedios son exhibidos por los insectos de Hidalgo, Veracruz, Lanquín, Petén y Bélice, mientras que en los especímenes de Oaxaca 2 solo comprende el $1.4 \%$ de los HC.

Considerando la fracción de $\mathrm{HC}$ metil ramificados, sobresale en los especímenes de Costa Rica la mayor abundancia de los compuestos con KI 3088 y KI 3288, con valores de abundancia relativa del 1 al $2.2 \%$ de los $\mathrm{HC}$ totales; mientras que en los ejemplares de Oaxaca 2 la alta abundancia de los HC con KI 3755 y KI 3779 (aproximadamente 4.5 \%). Los insectos de Lanquín y Bélice presentan de manera característica una alta proporción de los HC mono, di y trimetilramificados con 37 y 39 carbonos en la cadena lineal.

\section{2. 2. Análisis taxonómico basado en el patrón de hidrocarburos cuticulares.}

Aquí se presentan los resultados obtenidos en los análisis preliminares mediante PCA, así como en los análisis mediante DA.

\section{$\underline{\text { PCA de las distintas poblaciones de } T \text {. dimidiata }}$}

Los especímenes de Bélice y Honduras se analizaron en conjunto con las dos poblaciones de Colombia dado que esos países tenían una sola población cada uno. Con el resto de las poblaciones se hizo el análisis en función de la correspondencia a cada país.

En la figura 48 se muestra el gráfico de puntajes canónicos correspondiente al PCA realizado sobre los insectos de Bélice, Colombia y Honduras. 
Figura 48. Gráfico de puntajes canónicos correspondiente a los insectos de Bélice, Colombia y Honduras.

PCA case scores

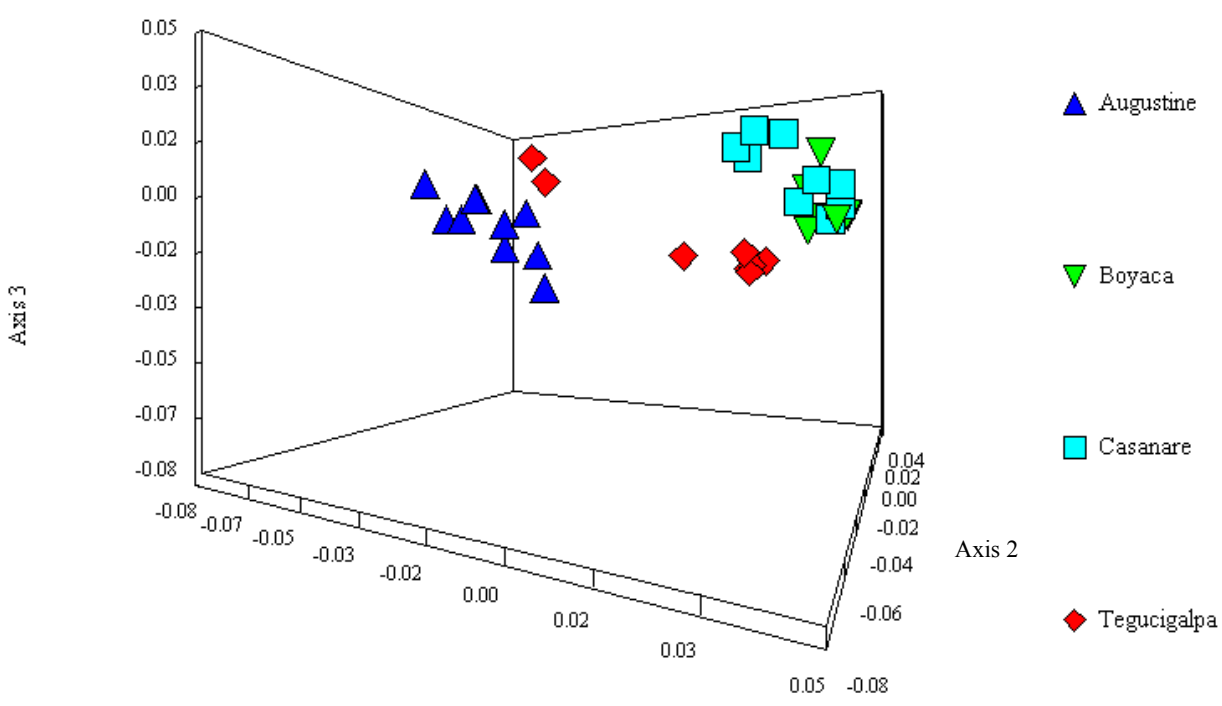

Axis 1

Se puede ver una alta homogeneidad entre las dos poblaciones de Colombia así como dentro de la población de Bélice.

En la figura 49 se muestra el gráfico de puntajes canónicos correspondiente al PCA realizado sobre los insectos de Costa Rica.

Figura 49. Gráfico de puntajes canónicos correspondiente a los insectos de Costa Rica.

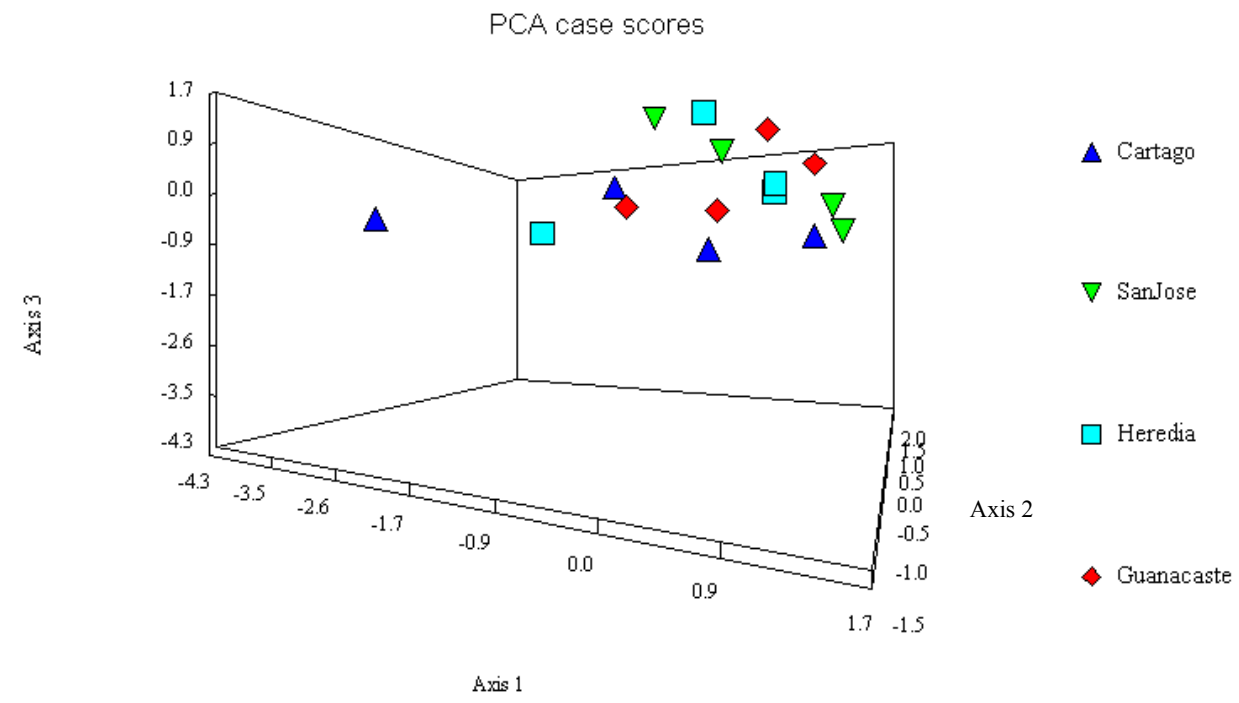

No se observa una clara diferenciación entre los insectos colectados en las distintas localidades. Hay un insecto “outlier” perteneciente al departamento de Cartago. 
En la figura 50 se muestra el gráfico de puntajes canónicos correspondiente al PCA realizado sobre los insectos de Guatemala.

Figura 50. Gráfico de puntajes canónicos correspondiente a los insectos de Guatemala.

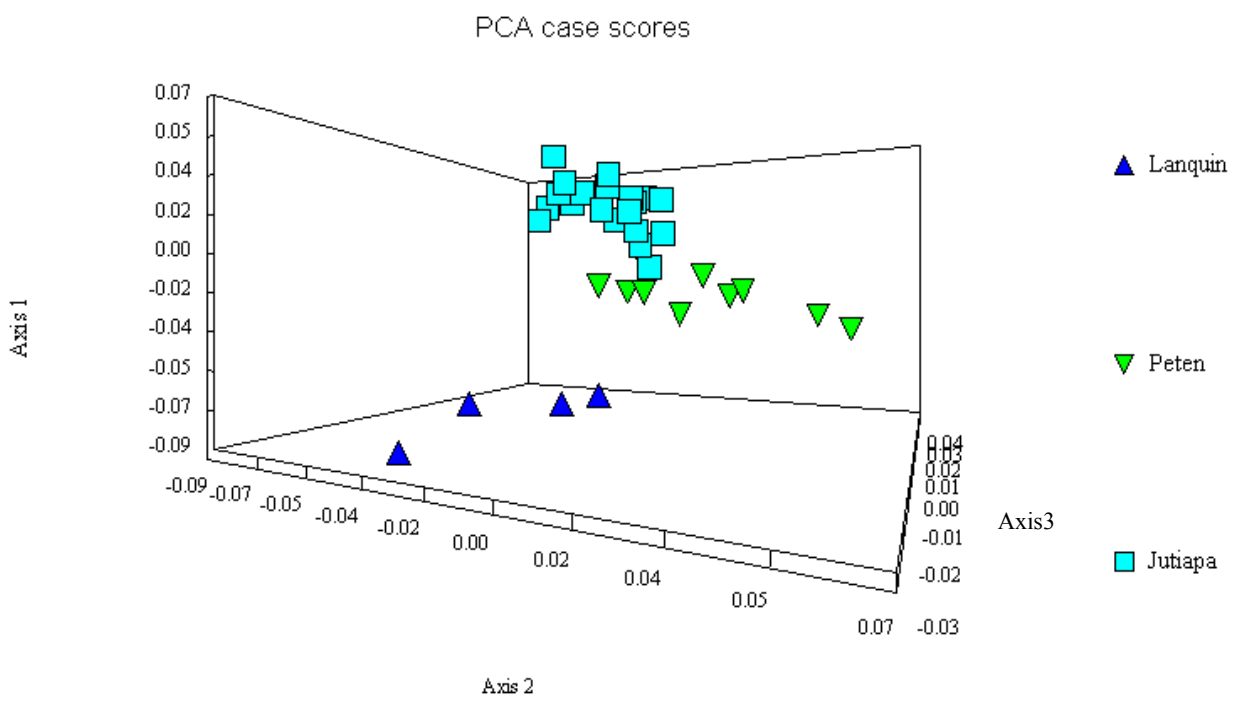

Se puede observar en estas poblaciones una diferenciación evidente de los ejemplares de Lanquín y menos evidente aunque aparente entre los de Jutiapa y Petén.

En la figura 51 se muestra el gráfico de puntajes canónicos correspondiente al PCA realizado sobre los insectos de Mexico.

Figura 51. Gráfico de puntajes canónicos correspondiente a los insectos de Mexico.

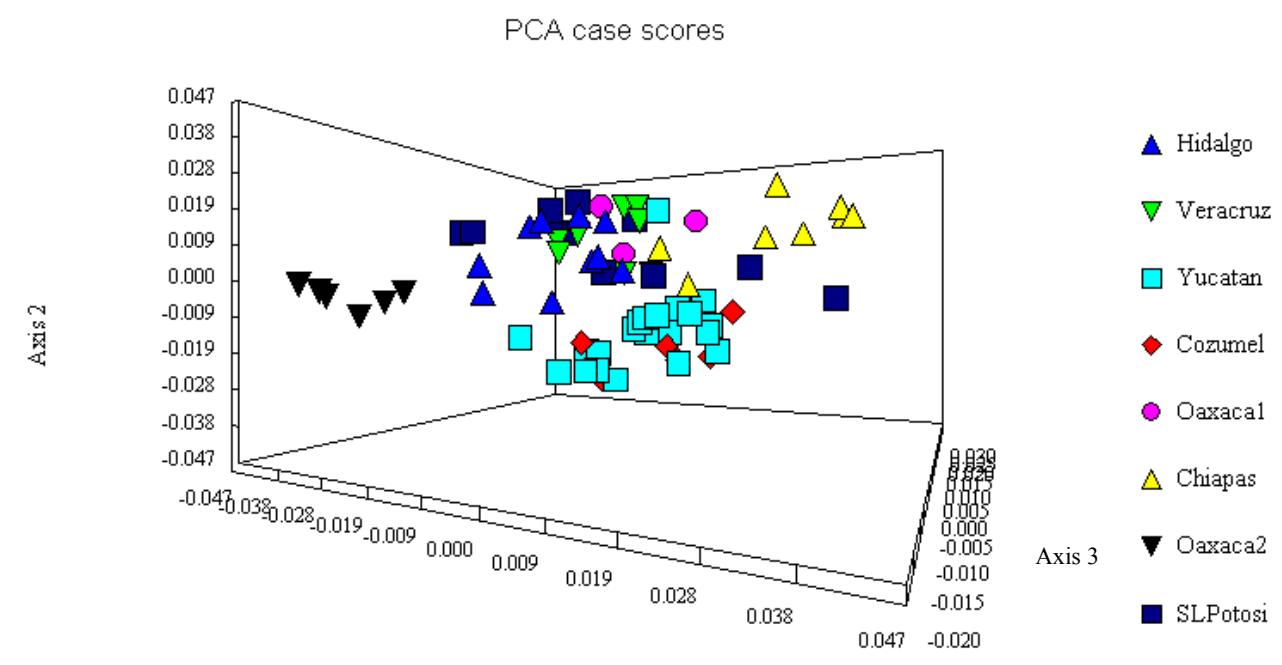

Axis 1 
Los ejemplares de Oaxaca 2 se distinguen con claridad de los de la región oriental del departamento y del resto de los especímenes. A su vez hay una separación moderada de los insectos de Yucatán y Cozumél respecto al resto de las poblaciones. Se detectó la presencia de outliers en la población de San Luis Postosí.

Se quitaron los ejemplares outliers reconocidos y posteriormente se hizo un PCA del conjunto de todas las poblaciones de la especie, el cual se muestra en la figuras 52.

Figura 52. Gráfico de puntajes canónicos de las poblaciones estudiadas de $T$. dimidiata. Eje 1 en la abcisa y eje 2 en la ordenada.

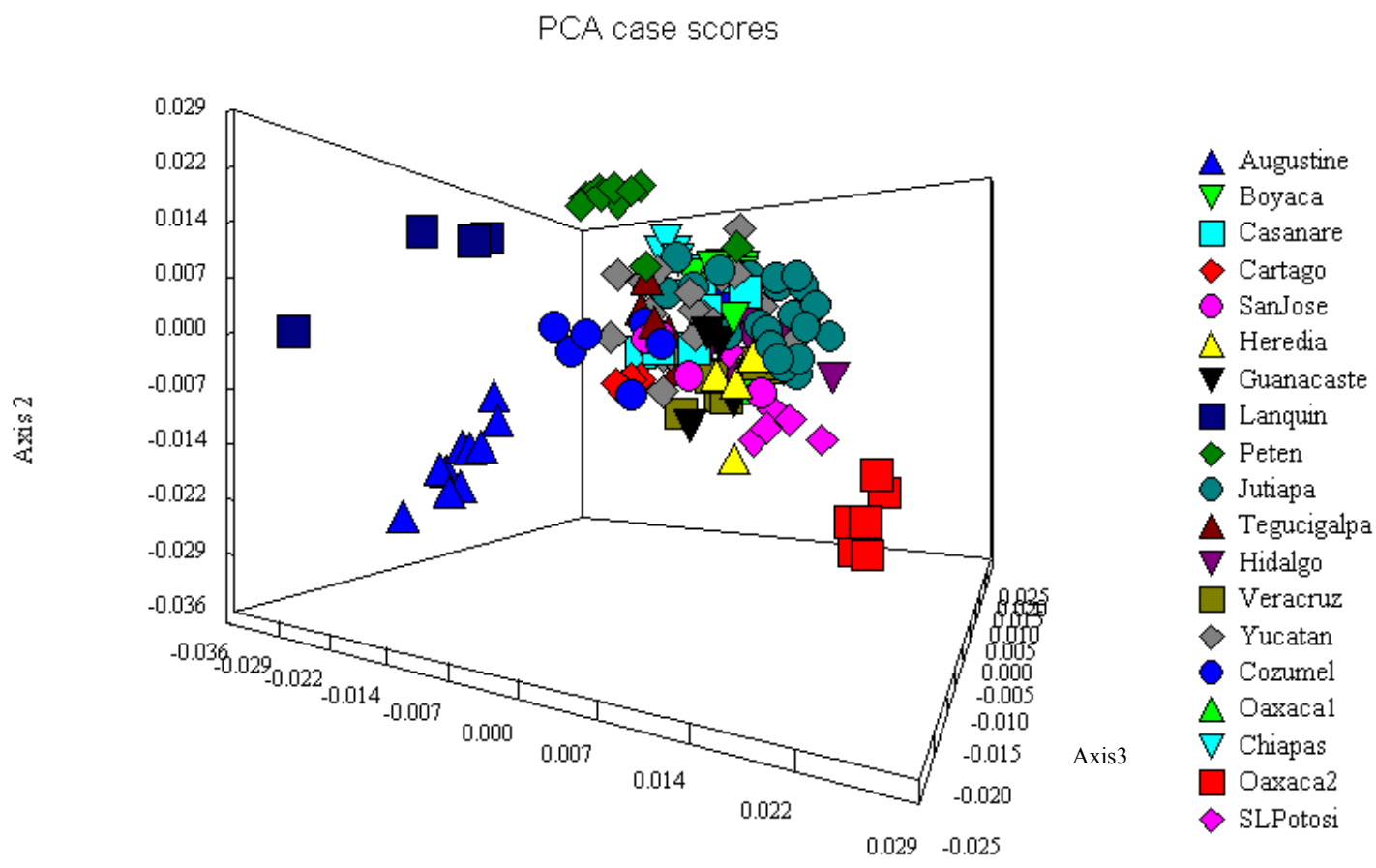

Axis 1

HC con mayor contribución relativa (KIs): 2500, 2700, 2900, 3087, 3100, 3288, 3300, 3489, 3522, 3580, 3630, $3655,3678,3686,3755,3779,3850,3854,3871,3894,3914,3980$

Se puede ver en la figura que la mayor parte de las poblaciones analizadas se agrupan de manera conjunta, sin embargo las poblaciones de Bélice, Oaxaca 2, Lanquín y Petén se separan claramente de ese grupo común.

A fin de ver de manera más detallada y precisa las relaciones entre los insectos se calculó el valor del centroide de cada población, a partir de los puntajes canónicos obtenidos en el PCA y se analizaron sus relaciones de similitud mediante un UPGMA basado en la distancia promedio (figura 53). 
Figura 53. Dendrograma de las poblaciones estudiadas de $T$. dimidiata basado en los puntajes canónicos del PCA.

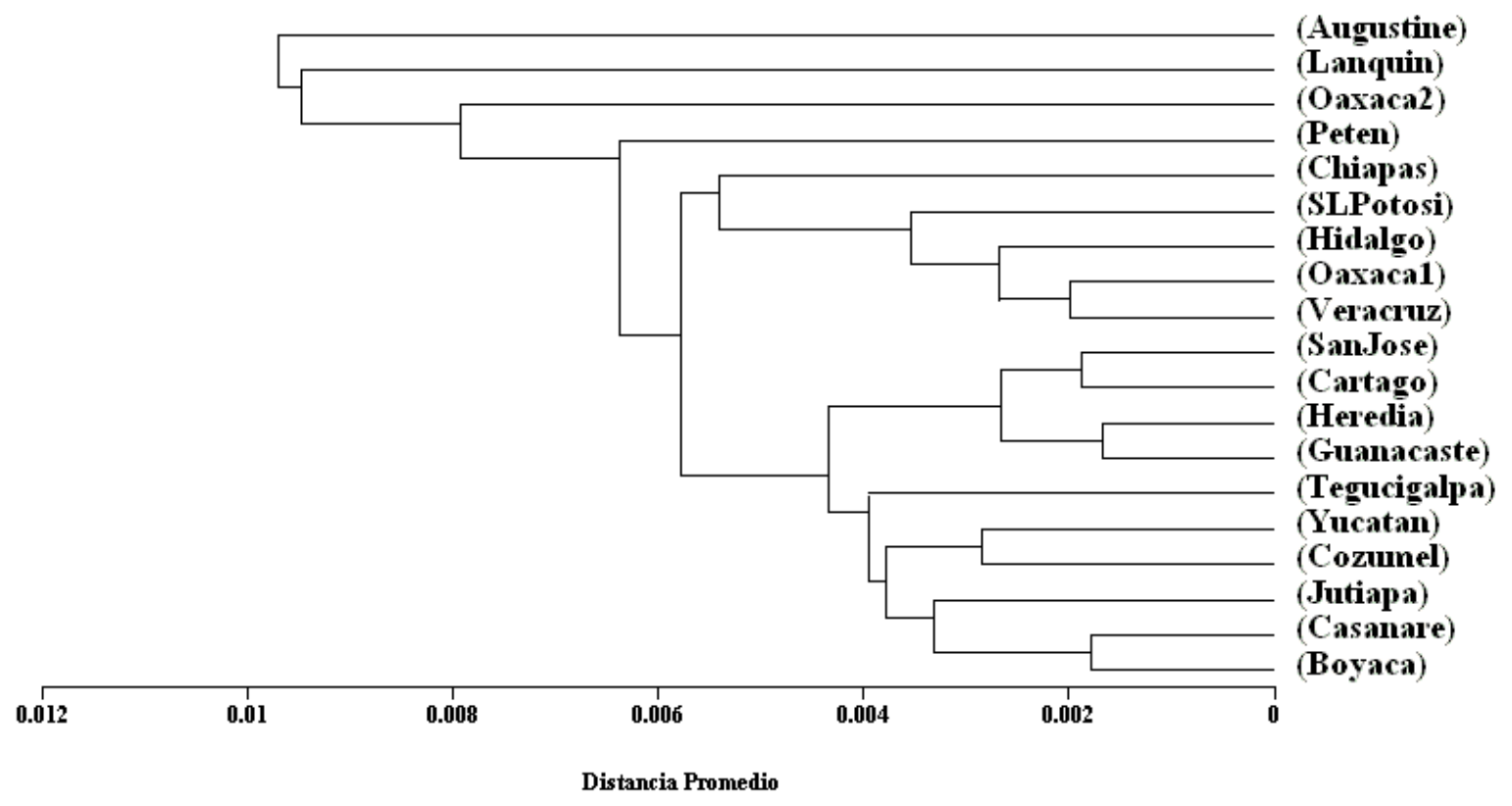

Se pueden reconocer tres grupos mayoritarios: uno formado por los insectos de la región central y oriental de Mexico, sumados los del departamento de Chiapas; otro por los de Costa Rica y un tercero que nuclea a los ejemplares mexicanos de la Península de Yucatán, Honduras, sur de Guatemala y las dos poblaciones de Colombia. La poblaciones de Petén y Oaxaca 2 muestran una notoria diferenciación y más aún las de Lanquín y Bélice.

\section{DA sobre las poblaciones de T. dimidiata.}

Basado en la similitud del resultado obtenido en el subcomplejo T. sordida, al analizar tanto las poblaciones agrupadas como por separado, se analizaron las poblaciones de $T$. dimidiata por separado aún con el bajo número de ejemplares en algunas localidades. Se empleó el método de eliminación hacia atrás como mejor criterio de selección de variables, obteniéndose 8 HC como variables que mejor discriminan entre los grupos (KIs 2900, 3300, $3522,3720,3800,3850,3871$ y 3980)con un $\lambda$ de Wilks altamente significativo ( $\mathrm{p} \leq 0.001)$. En la tabla 31 se computan los autovalores, varianzas parcial y acumulada y correlaciones canónicas de las funciones discriminantes obtenidas. 
Tabla 31. Características de las funciones discriminantes obtenidas en el análisis de $T$. dimidiata.

\begin{tabular}{|l|r|r|r|r|}
\multicolumn{5}{|c|}{ Autovalores } \\
\hline Función & Autov alor & \% de varianza & $\%$ acumulado & $\begin{array}{c}\text { Correlación } \\
\text { canónica }\end{array}$ \\
\hline 1 & $12.721^{\mathrm{a}}$ & 37.5 & 37.5 & .963 \\
2 & $8.321^{\mathrm{a}}$ & 24.5 & 62.1 & .945 \\
3 & $4.655^{\mathrm{a}}$ & 13.7 & 75.8 & .907 \\
4 & $3.845^{\mathrm{a}}$ & 11.3 & 87.1 & .891 \\
5 & $2.111^{\mathrm{a}}$ & 6.2 & 93.4 & .824 \\
6 & $1.730^{\mathrm{a}}$ & 5.1 & 98.5 & .796 \\
7 & $.382^{\mathrm{a}}$ & 1.1 & 99.6 & .526 \\
8 & $.137^{\mathrm{a}}$ & .4 & 100.0 & .348 \\
\hline
\end{tabular}

a. Se han empleado las 8 primeras funciones discriminantes canónicas en el análisis.

En las tablas 32 y 33 se muestran la matriz de estructura y el valor de las funciones discriminantes en los centroides de los grupos, mientras que en la figura 54 a, b y c se grafican los especímenes sobre las tres primeras funciones discriminantes, que acumulan un $76 \%$ de la varianza total.

Tabla 32. Matriz de estructura de las funciones discriminantes obtenidas en el análisis de T.dimidiata.

Matriz de estructura

\begin{tabular}{|c|c|c|c|c|c|c|c|c|}
\hline & \multicolumn{8}{|c|}{ Función } \\
\hline & 1 & 2 & 3 & 4 & 5 & 6 & 7 & 8 \\
\hline KI3522 & .236 & .228 & $-.587^{\star}$ & -.215 & -.106 & .523 & .424 & .194 \\
\hline $\mathrm{KI} 2900$ & -.042 & .217 & -.147 & $.755^{\star}$ & .132 & -.074 & -.305 & .494 \\
\hline $\mathrm{KI} 3300$ & -.060 & -.008 & .300 & -.536 & $.687^{*}$ & .252 & -.219 & -.185 \\
\hline KI3850 & -.210 & -.354 & .301 & .196 & -.356 & $.649^{*}$ & .300 & -.253 \\
\hline KI3720 & .038 & .360 & .207 & -.163 & -.134 & .208 & $.777^{*}$ & .368 \\
\hline KI3980 & -.382 & -.295 & -.263 & .151 & .222 & .104 & $.718^{*}$ & -.314 \\
\hline $\mathrm{KI} 3800$ & .462 & -.559 & .175 & .003 & .017 & .101 & $.631^{*}$ & .189 \\
\hline KI3871 & .190 & .225 & .083 & .293 & .085 & .254 & .362 & $-.786^{*}$ \\
\hline
\end{tabular}

Correlaciones intra-grupo combinadas entre las variables discriminantes y las funciones discriminantes canónicas tipificadas

Variables ordenadas por el tamaño de la correlación con la función.

*. May or correlación absoluta entre cada variable y cualquier función discriminante. 
Tabla 33. Valores de las funciones discriminantes en los centroides de los grupos de $T$. dimidiata .

Funciones en los centroides de los grupos

\begin{tabular}{|c|c|c|c|c|c|c|c|c|}
\hline \multirow[b]{2}{*}{ LOCAL } & \multicolumn{8}{|c|}{ Función } \\
\hline & 1 & 2 & 3 & 4 & 5 & 6 & 7 & 8 \\
\hline Augus tine & 11.104 & -3.193 & 1.159 & -1.183 & -.482 & -.568 & .115 & -.001 \\
\hline Boy aca & -.059 & 1.428 & 3.021 & 1.368 & 1.605 & 1.108 & -.686 & -.394 \\
\hline Casanare & .172 & 1.828 & 2.513 & 1.358 & .750 & 2.033 & -.119 & -.208 \\
\hline Cartago & -.401 & .175 & -.938 & 2.735 & -.253 & 1.367 & 1.508 & .164 \\
\hline SanJose & .353 & -.096 & -.268 & 2.540 & .310 & 1.217 & .651 & .914 \\
\hline Heredia & -1.136 & .614 & .991 & 3.097 & -.622 & -.012 & .143 & .130 \\
\hline Guanacaste & -.388 & .939 & .330 & 3.000 & -.591 & .219 & -.447 & -.221 \\
\hline Lanquin & -2.012 & 5.695 & 6.193 & -3.361 & -5.029 & .350 & .564 & .436 \\
\hline Peten & .169 & 5.639 & -2.523 & -2.949 & .224 & -.921 & -.045 & -.398 \\
\hline Jutiapa & -.458 & 1.331 & .068 & 1.504 & -.043 & -1.995 & -.396 & .119 \\
\hline Tegucigalpa & -.516 & -.380 & .992 & 2.269 & .932 & -1.735 & 1.448 & -.233 \\
\hline Hidalgo & -1.292 & -2.836 & .742 & -.648 & -.140 & -.156 & -.944 & -.006 \\
\hline Veracruz & -1.909 & -3.733 & .398 & -1.291 & -.345 & .325 & -.007 & -.501 \\
\hline Yucatan & .936 & .839 & -2.388 & -.389 & .024 & 1.446 & -.268 & .325 \\
\hline Cozumel & 1.553 & 1.128 & -2.640 & .432 & .304 & .505 & .920 & -.486 \\
\hline Oaxaca1 & -4.090 & -3.187 & 1.385 & -1.580 & .800 & 1.213 & .055 & -.634 \\
\hline Chiapas & -2.928 & -1.541 & 1.267 & -2.958 & 3.325 & -.864 & .283 & .617 \\
\hline Oaxaca2 & -3.387 & -4.477 & -3.178 & 1.375 & -2.803 & -.483 & -.302 & .004 \\
\hline SanLuis Potosi & -4.790 & -3.467 & -.126 & -1.842 & -.896 & .170 & .673 & -.058 \\
\hline
\end{tabular}

Funciones discriminantes canónicas no tipificadas ev aluadas en las medias de los grupos

Figura 54a. Gráfico de puntajes canónicos de los ejemplares de $T$. dimidiata sobre las 3 primeras funciones discriminantes.. FD 2 en la abcisa y FD 1 en la ordenada.
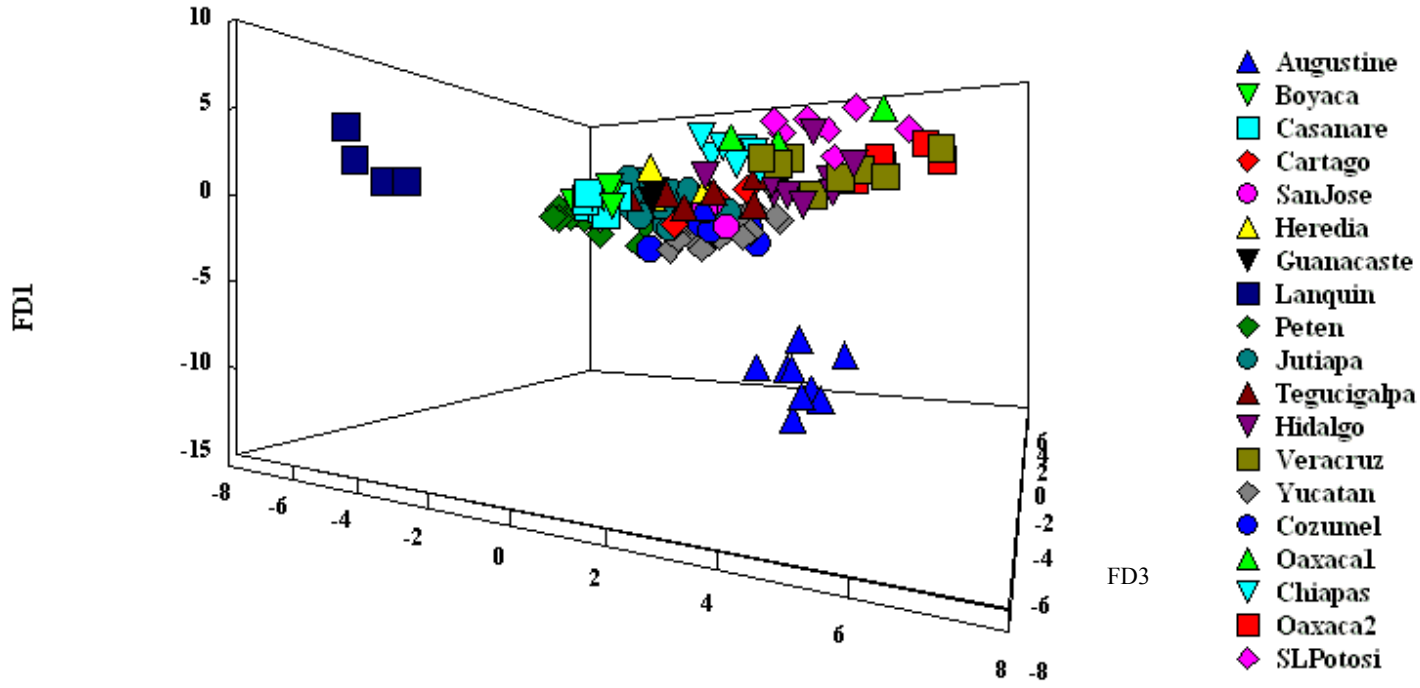
Figura 54b. Gráfico de puntajes canónicos de los ejemplares de $T$. dimidiata sobre las 3 primeras funciones discriminantes.. FD 1 en la abcisa y FD 2 en la ordenada.

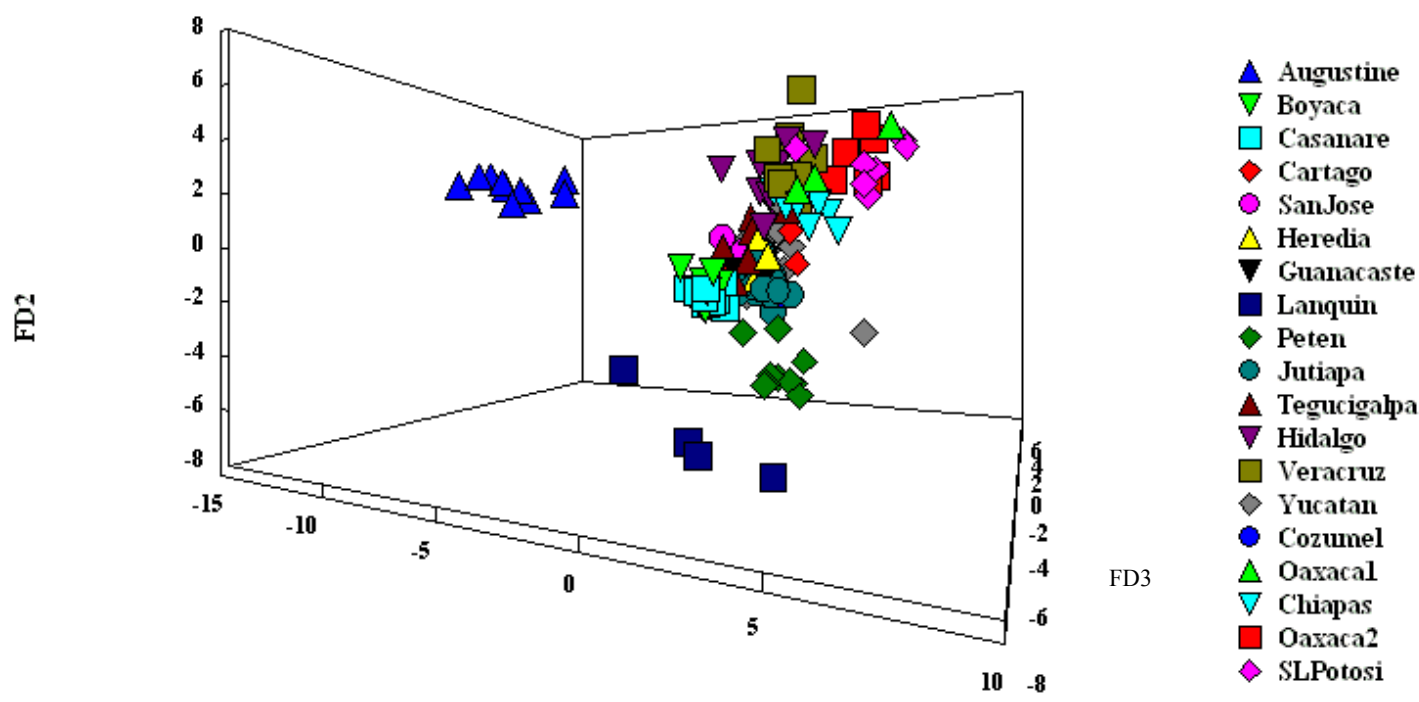

FD1

Figura 54c. Gráfico de puntajes canónicos de los ejemplares de $T$. dimidiata sobre las 3 primeras funciones discriminantes.. FD 2 en la abcisa y FD 3 en la ordenada.
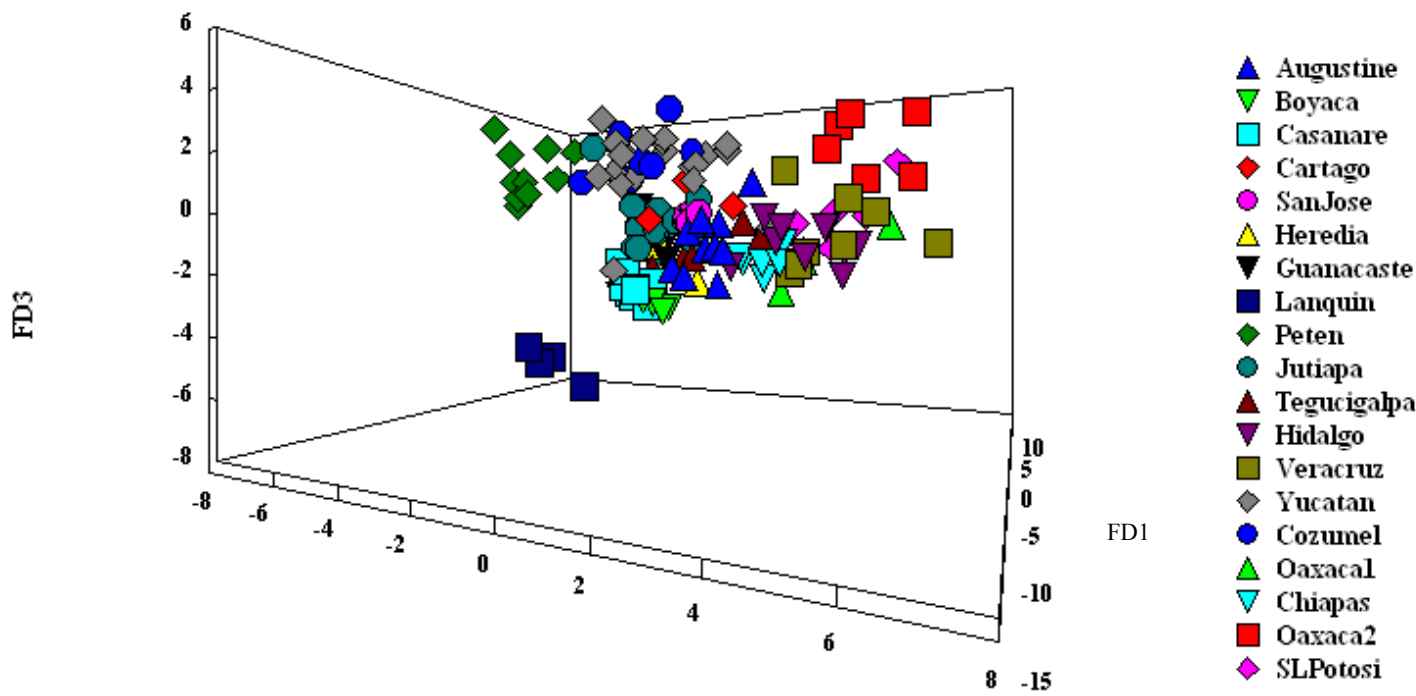

FD2

La FD 1, influenciada principalmente por los HC con KI 3800, 3980, 3522 y 3850 , contribuye principalmente a la diferenciación de los especímenes de Bélice, y en menor medida los de Yucatán y Cozumél del resto de las poblaciones mexicanas; estos grupos adoptan valores positivos de la función discriminante frente al resto de las poblaciones con 
valores en su mayoría negativos. La FD 2, influenciada mayormente por el HC KI 3800 y en menor medida por los $\mathrm{HC}$ con KI 3720 y 3850, contribuye a la separación de los insectos de Lanquín y Petén con valores positivos extremos y, a la diferenciación de las poblaciones mexicanas (excepto Yucatán y Cozumél), con valores altamente negativos, de los insectos centroamericanos y sudamericanos con valores mayormente positivos. La FD 3, influenciada principalmente por el HC KI 3522 y en menor medida por los HC con 3300 y 3850 contribuye a la separación de los insectos de Lanquín, con valores positivos extremos; a la diferenciación de los especimenes de Oaxaca 2, con alto valor negativo, respecto al resto de los insectos mexicanos; a la separación de los de Colombia, con valores positivos extremos, respecto a los centroamericanos. Adicionalmente esta FD contribuye en la separación de los insectos de Yucatán y Cozumél del resto de los ejemplares mexicanos y los de Petén (Guatemala) de las poblaciones geográficamente vecinas de Jutiapa (Guatemala) y Tegucigalpa (Honduras).La FD 4 influenciada principalmente por el HC con KI 2900 y en menor medida por el KI 3300, contribuye a la diferenciación de los ejemplares de Sur y Centro América respecto a los de Mexico y dentro de este país diferencia al grupo Oaxaca 2 respecto al resto de los insectos mexicanos. El resto de las funciones discriminantes se aplican a la separación de las poblaciones individuales dentro de los grupos más inclusivos.

Se realizó un dendrograma con el método UPGMA, basado en las distancias de Mahalanobis entre los centroides de los grupos derivadas a partir del DA (figura 55).

Figura 55. Dendrograma basado en la distancia de Mahalanobis entre las poblaciones de $T$. dimidiata.

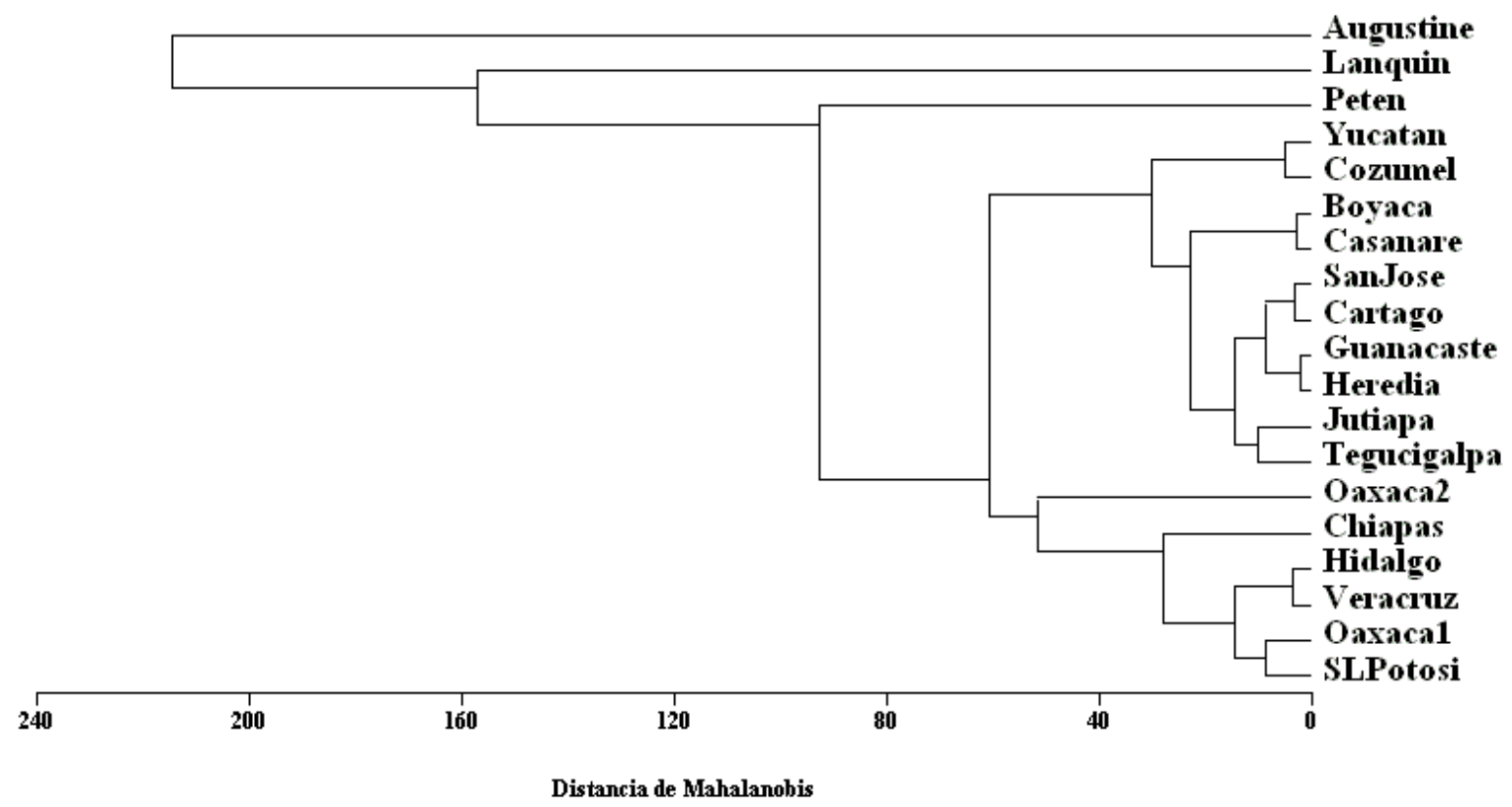


Un elevado grado de diferenciación respecto al resto de las poblaciones de la especie es exhibido por los insectos colectados en las cuevas de Lanquín y Bélice, y en menor medida, también por los especímenes de Petén. El resto de las poblaciones se encuentra organizado en dos grandes grupos, estructurados éstos principalmente en forma clinal en sentido norte a sur y donde la similitud entre las poblaciones se basa, salvo excepciones, en su proximidad geográfica: uno formado por la mayor parte de las poblaciones mexicanas, los ejemplares de la región central de Mexico (Oaxaca 1 y San Luis Potosí), los de la costa este (Hidalgo, Veracruz), región occidental (Oaxaca 2) y sur de Mexico (Chiapas); el segundo, formado por los ejemplares mexicanos de Yucatán e isla de Cozumél, las dos poblaciones de Colombia (Boyaca y Casanare) y el resto de las poblaciones analizadas correspondientes a los países centroamericanos de Guatemala (Jutiapa), Honduras (Tegucigalpa) y Costa Rica (Cartago, San José, Guanacaste y Heredia).

En los resultados de clasificación, $83 \%$ de los insectos resultaron correctamente clasificados dentro de sus poblaciones en la clasificación original, mientras que un $75 \%$ tras la validación cruzada de los casos.

Se probó además disminuir la rigurosidad del proceso de eliminación hacia atrás dentro del DA, con lo cuál ingresó un HC más al análisis. De esta manera 9 HC quedaron seleccionados como variables que mejor discriminan entre los grupos (KIs 2900, 2932, 3300, $3522,3720,3800,3850,3871$ y 3980); el $\lambda$ de Wilks resultó altamente significativo ( $\mathrm{p} \leq$ 0.001). En la tabla 34 se computan los autovalores, varianzas parcial y acumulada y correlaciones canónicas de este nuevo set de funciones discriminantes obtenidas.

Tabla 34. Características de las funciones discriminantes obtenidas en el DA de T. dimidiata.

\begin{tabular}{|l|r|r|r|r|}
\hline \multicolumn{5}{|c|}{ Autovalores } \\
\hline Función & Autov alor & $\%$ de varianza & $\%$ acumulado & $\begin{array}{c}\text { Correlación } \\
\text { canónica }\end{array}$ \\
\hline 1 & $13.295^{\mathrm{a}}$ & 35.9 & 35.9 & .964 \\
2 & $8.807^{\mathrm{a}}$ & 23.7 & 59.6 & .948 \\
3 & $5.399^{\mathrm{a}}$ & 14.6 & 74.2 & .919 \\
4 & $4.476^{\mathrm{a}}$ & 12.1 & 86.2 & .904 \\
5 & $2.111^{\mathrm{a}}$ & 5.7 & 91.9 & .824 \\
6 & $1.737^{\mathrm{a}}$ & 4.7 & 96.6 & .797 \\
7 & $.862^{\mathrm{a}}$ & 2.3 & 98.9 & .680 \\
8 & $.261^{\mathrm{a}}$ & .7 & 99.6 & .455 \\
9 & $.137^{\mathrm{a}}$ & .4 & 100.0 & .347 \\
\hline
\end{tabular}


En las tablas 35 y 36 se muestran la matriz de estructura y el valor de las funciones discriminantes en los centroides de los grupos, mientras que en la figura 56 a, b y c se grafican los especímenes sobre las tres primeras funciones discriminantes, que acumulan un $74 \%$ de la varianza total.

Tabla 35. Matriz de estructura de las funciones discriminantes obtenidas en el análisis de T.dimidiata.

Matriz de estructura

\begin{tabular}{|l|l|l|l|l|l|l|l|r|r|}
\hline & \multicolumn{10}{|c|}{ Función } \\
\cline { 2 - 9 } & 1 & 2 & 3 & 4 & 5 & 6 & 7 & 8 & 9 \\
\hline KI3800 & -.399 & $.602^{*}$ & -.004 & -.169 & .017 & .116 & -.325 & .542 & .194 \\
KI2900 & .062 & -.130 & .452 & $.553^{*}$ & .131 & -.036 & -.194 & -.417 & .488 \\
KI3300 & .037 & -.045 & -.178 & -.541 & $.689^{\star}$ & .222 & .322 & -.101 & -.185 \\
KI3850 & .247 & .335 & .145 & -.180 & -.358 & $.676^{*}$ & -.302 & .186 & -.252 \\
KI2932 & .180 & .318 & .355 & .156 & -.231 & -.102 & $.616^{*}$ & .437 & -.287 \\
KI3720 & -.075 & -.347 & .089 & -.241 & -.134 & .205 & -.215 & $.749^{*}$ & .376 \\
KI3980 & .390 & .213 & -.205 & .250 & .221 & .127 & -.435 & $.591^{*}$ & -.309 \\
KI3522 & -.277 & -.242 & -.381 & .378 & -.105 & .495 & .128 & $.514^{*}$ & .200 \\
KI3871 & -.186 & -.148 & .303 & .113 & .084 & .272 & -.230 & .302 & $-.783^{*}$ \\
\hline
\end{tabular}

Correlaciones intra-grupo combinadas entre las variables discriminantes y las funciones discriminantes canónicas tipif icadas

Variables ordenadas por el tamaño de la correlación con la función.

*. May or correlación absoluta entre cada variable y cualquier función discriminante.

Tabla 36. Valores de las funciones discriminantes en los centroides de los grupos de $T$. dimidiata .

Funciones en los centroides de los grupos

\begin{tabular}{|c|c|c|c|c|c|c|c|c|c|}
\hline \multirow[b]{2}{*}{ LOCAL } & \multicolumn{9}{|c|}{ Función } \\
\hline & 1 & 2 & 3 & 4 & 5 & 6 & 7 & 8 & 9 \\
\hline Augus tine & -10.860 & 4.260 & -.081 & -1.575 & -.479 & -.611 & .185 & .181 & .001 \\
\hline Boy aca & .367 & -.711 & 3.420 & -1.416 & 1.602 & 1.144 & .243 & -.577 & -.400 \\
\hline Casanare & .213 & -.958 & 3.474 & -.900 & .748 & 2.021 & .606 & .220 & -.202 \\
\hline Cartago & 1.015 & .599 & 2.128 & 2.594 & -.257 & 1.401 & -.242 & 1.651 & .184 \\
\hline SanJose & .364 & 1.045 & 2.518 & 1.995 & .308 & 1.225 & .284 & .954 & .927 \\
\hline Heredia & 1.800 & .280 & 3.547 & 1.269 & -.627 & .061 & -.185 & .208 & .133 \\
\hline Guanacaste & .740 & -.278 & 2.776 & 1.619 & -.598 & .323 & -.437 & -.604 & -.228 \\
\hline Lanquin & .711 & -6.518 & 1.128 & -7.072 & -5.030 & .379 & -1.004 & -.001 & .431 \\
\hline Peten & -1.434 & -6.407 & -2.791 & .459 & .234 & -1.090 & 1.039 & .319 & -.392 \\
\hline Jutiapa & .794 & -.722 & 2.015 & 1.117 & -.041 & -2.008 & .467 & -.109 & .120 \\
\hline Tegucigalpa & .240 & .107 & .951 & .091 & .919 & -1.424 & -3.435 & -.006 & -.246 \\
\hline Hidalgo & 1.674 & 2.670 & -.526 & -1.074 & -.139 & -.185 & .561 & -.766 & -.013 \\
\hline Veracruz & 2.434 & 3.468 & -1.213 & -1.161 & -.341 & .247 & .746 & .356 & -.494 \\
\hline Yucatan & -1.476 & -1.296 & -2.007 & 1.542 & .023 & 1.449 & -.127 & -.450 & .318 \\
\hline Cozumel & -2.143 & -1.490 & -1.642 & 2. 184 & .301 & .584 & -1.198 & .385 & -.487 \\
\hline Oaxaca1 & 4.135 & 2.227 & -1.721 & -2.435 & .798 & 1.246 & -.473 & -.218 & -.639 \\
\hline Chiapas & 2.615 & .492 & -2.377 & -3.041 & 3.329 & -.896 & -.202 & .134 & .617 \\
\hline Oaxaca2 & 4.039 & 4.107 & -1.789 & 3.144 & -2.804 & -.451 & -.060 & -.304 & .001 \\
\hline SanLuis Potosi & 5.007 & 2.514 & -2.464 & -1.249 & -.893 & .125 & .055 & .740 & -.050 \\
\hline
\end{tabular}

Funciones discriminantes canónicas no tipificadas ev aluadas en las medias de los grupos 
Figura 56a. Gráfico de puntajes canónicos de los ejemplares de $T$. dimidiata sobre las 3 primeras funciones discriminantes. FD 2 en la abcisa y FD 1 en la ordenada.

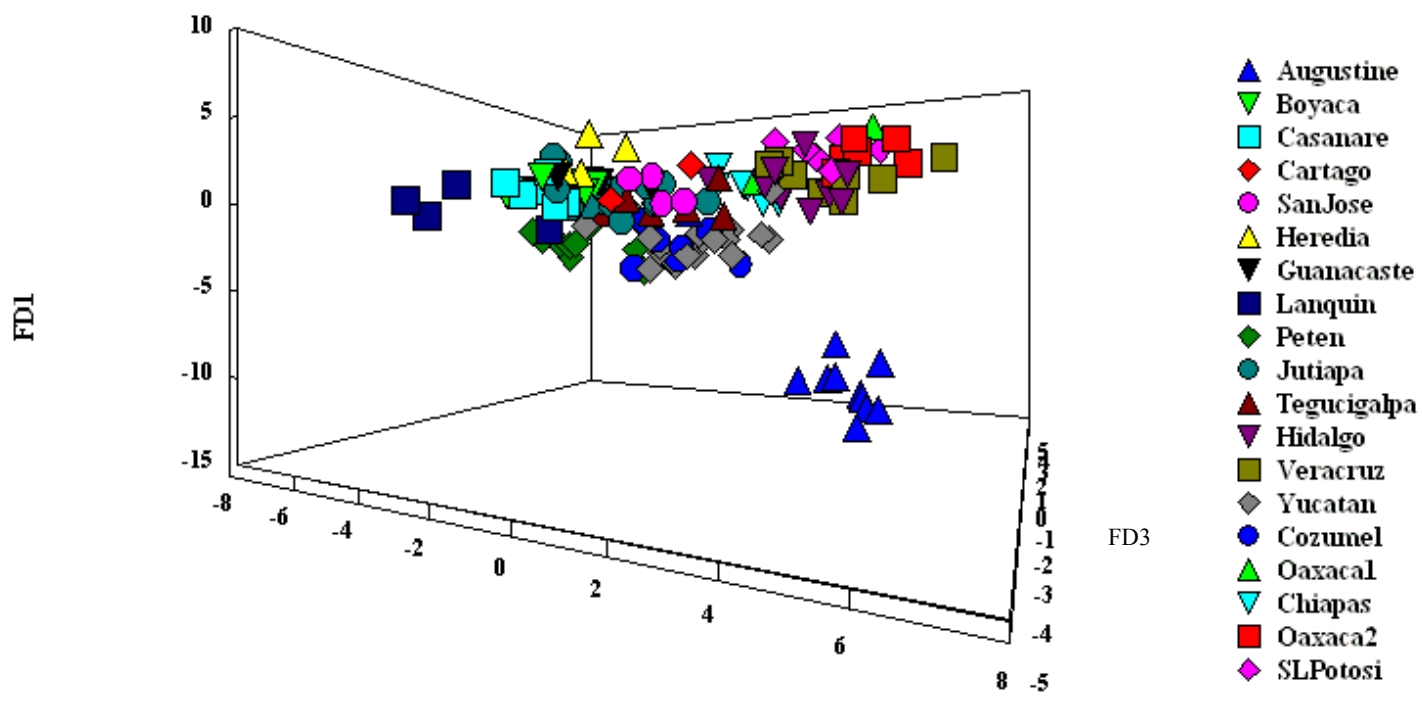

FD2

Figura 56b. Gráfico de puntajes canónicos de los ejemplares de $T$. dimidiata sobre las 3 primeras funciones discriminantes. FD 1 en la abcisa y FD 2 en la ordenada.
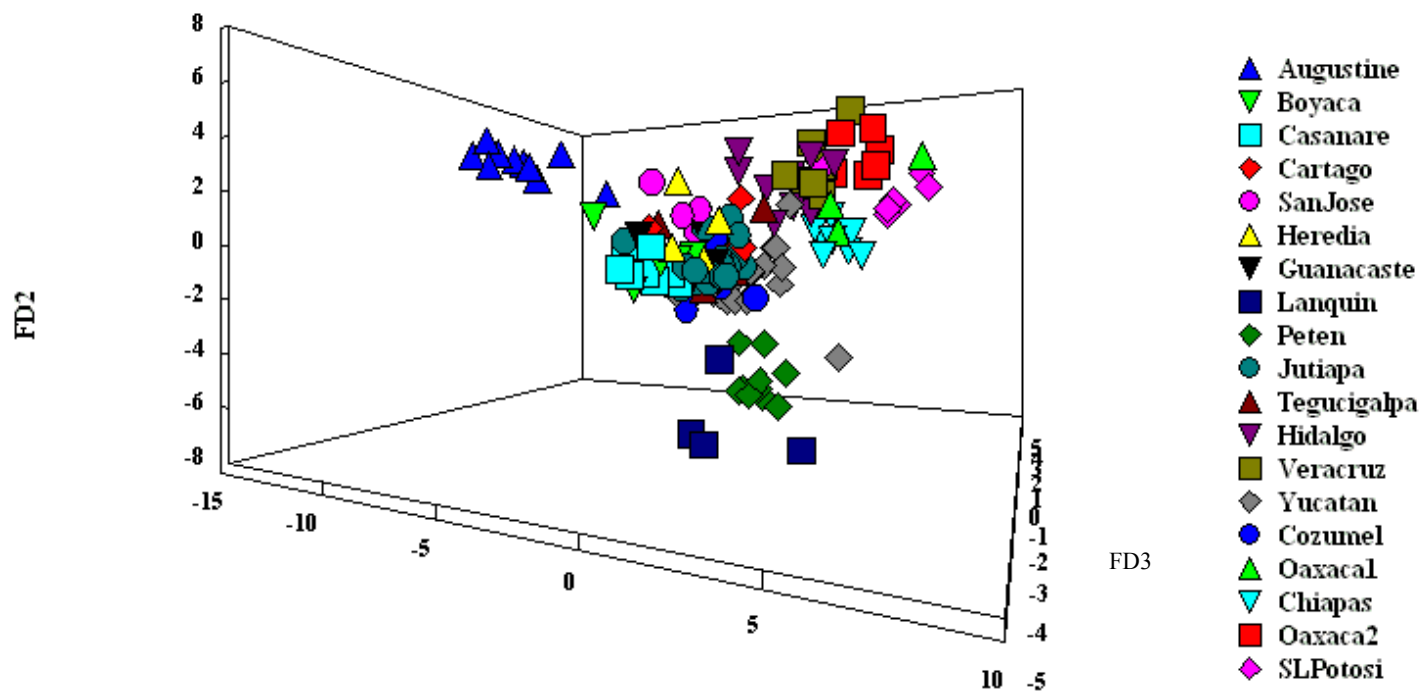

FD1 
Figura 56c. Gráfico de puntajes canónicos de los ejemplares de $T$. dimidiata sobre las 3 primeras funciones discriminantes. FD 2 en la abcisa y FD 3 en la ordenada.

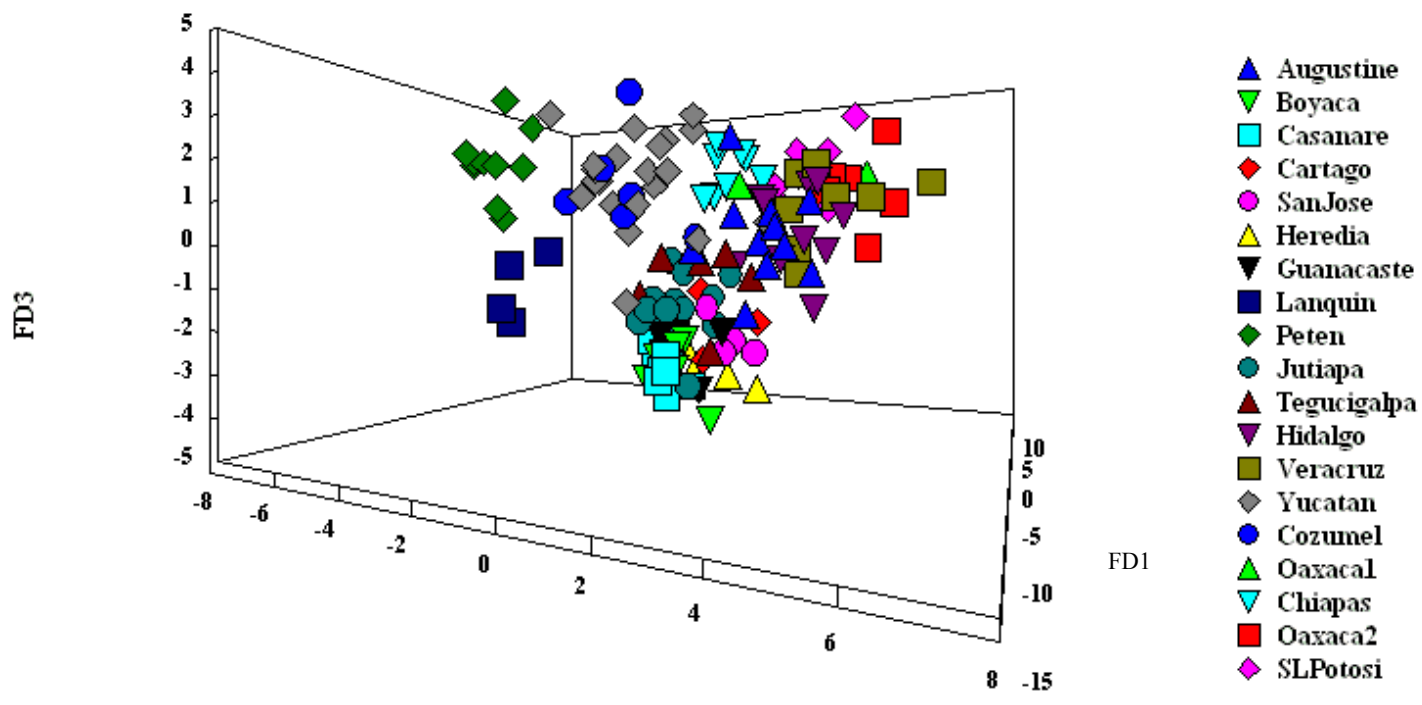

FD2

La FD 1, influenciada principalmente por los HC con KI 3800, 3980, 3522 y 3850, contribuye principalmente a la diferenciación de los especímenes de Bélice, y en menor medida los de Petén, Yucatán y Cozumél; estos grupos adoptan valores negativos de la función discriminante frente al resto de las poblaciones con valores mayormente positivos. La FD 2, influenciada mayormente por el HC KI 3800 y en menor medida por los HC con KI 3720, 3850 y 2932, contribuye a la separación de los insectos de Bélice respecto a los de Lanquín, Petén, Cozumél y Yucatán; además discrimina a estas dos últimas poblaciones mencionadas del resto de los especímenes mexicanos. La FD 3, influenciada principalmente por los HC con KI 2900, 3522, 2932 y 3871 contribuye a la separación de los insectos de Centro y Sur América respecto a los especímenes mexicanos. La FD 4 influenciada principalmente por el HC con KI 2900 y en menor medida por el KI 3300, contribuye a una gran diferenciación de los insectos de Lanquín así como la diferenciación entre los ejemplares de Sur y Centro América y los de Oaxaca 2 respecto al resto de los insectos mexicanos. El resto de las funciones discriminantes se aplican a la separación de las poblaciones individuales dentro de los grupos más inclusivos.

El dendograma (UPGMA) basado en las distancias de Mahalanobis entre los centroides de los grupos se muestra en la figura 57. 
Figura 57. Dendrograma basado en la distancia de Mahalanobis entre las poblaciones de $T$. dimidiata.

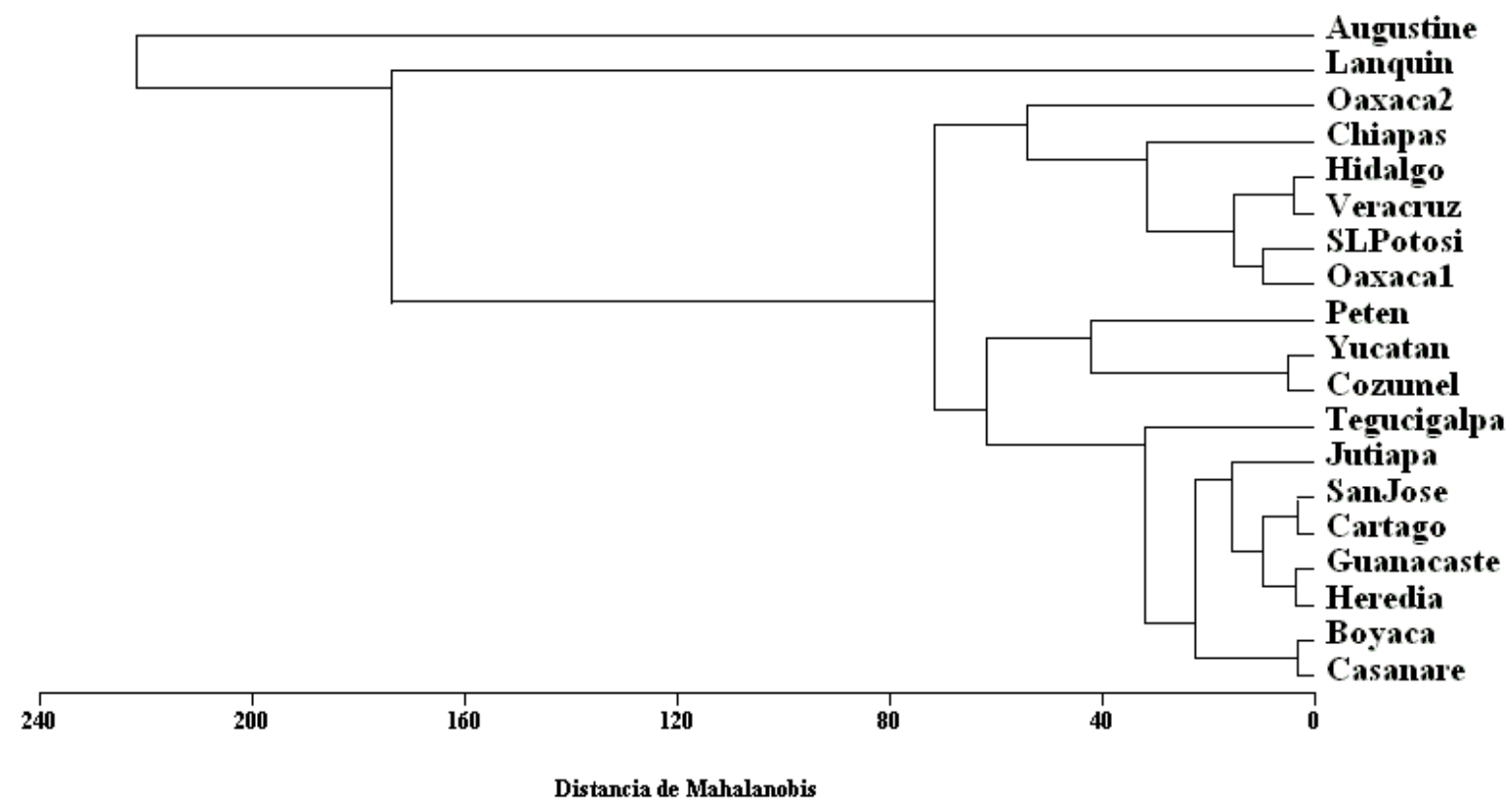

Dos poblaciones, las colectadas en las cuevas de Lanquín y Bélice, muestran un elevado grado de diferenciación respecto al resto de las poblaciones de la especie, que se encuentra organizado en tres grandes grupos estructurados éstos mayormente en forma clinal, en sentido norte a sur: uno formado por la mayor parte de las poblaciones mexicanas, los ejemplares de la región central de Mexico (Oaxaca 1 y San Luis Potosí), los de la costa este (Hidalgo, Veracruz), región occidental (Oaxaca 2) y sur de Mexico (Chiapas); el segundo, que no sigue un patrón clinal, formado por los ejemplares mexicanos de Yucatán e isla de Cozumél y los del norte de Guatemala (Petén); y el tercero que está integrado por los especímenes del sur de Guatemala (Jutiapa), Honduras (Tegucigalpa), las cuatro poblaciones analizadas de Costa Rica (Cartago, San José, Guanacaste y Heredia) y las dos de Colombia (Boyaca, Casanare) en América del Sur.

En la tabla 37 se presentan los resultados de clasificación mediante validación cruzada para cada población así como los resultados totales. 
Tabla 37. Resultados de clasificación de los grupos analizados de T. dimidiata (\%).

\begin{tabular}{|c|c|c|c|c|c|}
\hline \multirow[b]{3}{*}{ Grupo original } & \multicolumn{5}{|c|}{ Grupo de pertenencia pronosticado (\%) } \\
\hline & \multirow{2}{*}{$\begin{array}{c}\text { Bélice } \\
\text { Augustine } \\
\end{array}$} & \multicolumn{4}{|c|}{ Costa Rica } \\
\hline & & Cartago & SanJose & Heredia & Guanacaste \\
\hline Augustine & 100 & 0 & 0 & 0 & 0 \\
\hline Boyaca & 0 & 0 & 0 & 0 & 0 \\
\hline Casanare & 0 & 0 & 0 & 0 & 0 \\
\hline Cartago & 0 & 33.3 & 66.7 & 0 & 0 \\
\hline SanJose & 0 & 25 & 75 & 0 & 0 \\
\hline Heredia & 0 & 0 & 0 & 25 & 50 \\
\hline Guanacaste & 0 & 0 & 25 & 25 & 25 \\
\hline Lanquin & 0 & 0 & 0 & 0 & 0 \\
\hline Peten & 0 & 0 & 0 & 0 & 0 \\
\hline Jutiapa & 0 & 0 & 4.8 & 0 & 0 \\
\hline Tegucigalpa & 0 & 0 & 0 & 0 & 0 \\
\hline Hidalgo & 0 & 0 & 0 & 0 & 0 \\
\hline Veracruz & 0 & 0 & 0 & 0 & 0 \\
\hline Yucatan & 0 & 0 & 0 & 0 & 0 \\
\hline Cozumel & 0 & 0 & 0 & 0 & 0 \\
\hline Oaxaca1 & 0 & 0 & 0 & 0 & 0 \\
\hline Chiapas & 0 & 0 & 0 & 0 & 0 \\
\hline Oaxaca2 & 0 & 0 & 0 & 0 & 0 \\
\hline San L Potosi & 0 & 0 & 0 & 0 & 0 \\
\hline
\end{tabular}

\begin{tabular}{|c|c|c|c|c|c|}
\hline \multirow[b]{3}{*}{ Grupo original } & \multicolumn{5}{|c|}{ Grupo de pertenencia pronosticado (\%) } \\
\hline & \multicolumn{2}{|c|}{ Colombia } & \multicolumn{3}{|c|}{ Guatemala } \\
\hline & Boyaca & Casanare & Lanquin & Peten & Jutiapa \\
\hline Augustine & 0 & 0 & 0 & 0 & 0 \\
\hline Boyaca & 85.7 & 14.3 & 0 & 0 & 0 \\
\hline Casanare & 33.3 & 66.7 & 0 & 0 & 0 \\
\hline Cartago & 0 & 0 & 0 & 0 & 0 \\
\hline SanJose & 0 & 0 & 0 & 0 & 0 \\
\hline Heredia & 0 & 0 & 0 & 0 & 25 \\
\hline Guanacaste & 0 & 0 & 0 & 0 & 25 \\
\hline Lanquin & 0 & 0 & 100 & 0 & 0 \\
\hline Peten & 0 & 0 & 0 & 100 & 0 \\
\hline Jutiapa & 0 & 0 & 0 & 0 & 95.2 \\
\hline Tegucigalpa & 0 & 0 & 0 & 0 & 0 \\
\hline Hidalgo & 0 & 0 & 0 & 0 & 10 \\
\hline Veracruz & 0 & 0 & 0 & 0 & 0 \\
\hline Yucatan & 0 & 4.8 & 0 & 4.8 & 0 \\
\hline Cozumel & 0 & 0 & 0 & 0 & 0 \\
\hline Oaxaca1 & 0 & 0 & 0 & 0 & 0 \\
\hline Chiapas & 0 & 0 & 0 & 0 & 0 \\
\hline Oaxaca2 & 0 & 0 & 0 & 0 & 0 \\
\hline San L Potosi & 0 & 0 & 0 & 0 & 0 \\
\hline
\end{tabular}




\begin{tabular}{|c|c|c|c|c|c|}
\hline \multirow[b]{3}{*}{ Grupo original } & \multicolumn{5}{|c|}{ Grupo de pertenencia pronosticado (\%) } \\
\hline & \multirow{2}{*}{$\frac{\text { Honduras }}{\text { Tegucigalpa }}$} & \multicolumn{4}{|c|}{ Mexico } \\
\hline & & Hidalgo & Veracruz & Yucatan & Cozumel \\
\hline Augustine & 0 & 0 & 0 & 0 & 0 \\
\hline Boyaca & 0 & 0 & 0 & 0 & 0 \\
\hline Casanare & 0 & 0 & 0 & 0 & 0 \\
\hline Cartago & 0 & 0 & 0 & 0 & 0 \\
\hline SanJose & 0 & 0 & 0 & 0 & 0 \\
\hline Heredia & 0 & 0 & 0 & 0 & 0 \\
\hline Guanacaste & 0 & 0 & 0 & 0 & 0 \\
\hline Lanquin & 0 & 0 & 0 & 0 & 0 \\
\hline Peten & 0 & 0 & 0 & 0 & 0 \\
\hline Jutiapa & 0 & 0 & 0 & 0 & 0 \\
\hline Tegucigalpa & 100 & 0 & 0 & 0 & 0 \\
\hline Hidalgo & 0 & 70 & 10 & 0 & 0 \\
\hline Veracruz & 12.5 & 50 & 37.5 & 0 & 0 \\
\hline Yucatan & 0 & 4.8 & 0 & 81 & 4.8 \\
\hline Cozumel & 0 & 0 & 0 & 57.1 & 42.9 \\
\hline Oaxaca1 & 0 & 0 & 33.3 & 0 & 0 \\
\hline Chiapas & 0 & 0 & 0 & 0 & 0 \\
\hline Oaxaca2 & 0 & 0 & 0 & 0 & 0 \\
\hline San L Potosi & 0 & 0 & 14.3 & 0 & 0 \\
\hline
\end{tabular}

\begin{tabular}{|c|c|c|c|c|}
\hline \multirow[b]{3}{*}{ Grupo original } & \multicolumn{4}{|c|}{ Grupo de pertenencia pronosticado (\%) } \\
\hline & \multicolumn{4}{|c|}{ Mexico } \\
\hline & Oaxaca1 & Chiapas & Oaxaca2 & SanLPotosi \\
\hline Augustine & 0 & 0 & 0 & 0 \\
\hline Boyaca & 0 & 0 & 0 & 0 \\
\hline Casanare & 0 & 0 & 0 & 0 \\
\hline Cartago & 0 & 0 & 0 & 0 \\
\hline SanJose & 0 & 0 & 0 & 0 \\
\hline Heredia & 0 & 0 & 0 & 0 \\
\hline Guanacaste & 0 & 0 & 0 & 0 \\
\hline Lanquin & 0 & 0 & 0 & 0 \\
\hline Peten & 0 & 0 & 0 & 0 \\
\hline Jutiapa & 0 & 0 & 0 & 0 \\
\hline Tegucigalpa & 0 & 0 & 0 & 0 \\
\hline Hidalgo & 10 & 0 & 0 & 0 \\
\hline Veracruz & 0 & 0 & 0 & 0 \\
\hline Yucatan & 0 & 0 & 0 & 0 \\
\hline Cozumel & 0 & 0 & 0 & 0 \\
\hline Oaxaca1 & 0 & 33.3 & 0 & 33.3 \\
\hline Chiapas & 0 & 100 & 0 & 0 \\
\hline Oaxaca2 & 0 & 0 & 100 & 0 \\
\hline San L Potosi & 0 & 0 & 0 & 85.7 \\
\hline
\end{tabular}


Los resultados de clasificación muestran una estructuración de la especie en grandes grupos, similar a la obtenida en el dendrograma a partir de las distancias de Mahalanobis, con un grado variable de mezcla dentro de ellos lo cual disminuye la eficiencia de la clasificación (86\% de los especímenes correctamente clasificados originalmente y $77 \%$ tras la validación cruzada de los casos). La mayor similitud entre especímenes, deducida a partir de los errores en la clasificación parcial, se puede observar entre las poblaciones de Colombia; las del este, centro y sur de Mexico (Veracruz, Hidalgo, San Luis Potosí, Oaxaca 1, Chiapas), y en América Central entre las de Costa Rica y el sur de Guatemala. 


\section{Discusión}

$\underline{\text { V. 1. Estructura de los hidrocarburos cuticulares en el subcomplejo T. sordida. }}$

La mezcla de cadenas lineales y metil ramificadas que compone la fracción de hidrocarburos de este grupo, presenta un patrón de complejidad que depende fundamentalmente de la especie en análisis y en segundo lugar de las poblaciones dentro de una misma especie.

Una serie de características del patrón de hidrocarburos cuticulares soportaría la inclusión de este subcomplejo dentro del complejo T. infestans. Además de la similitud en los perfiles se detectó la presencia de ciertos $\mathrm{HC}$ ya reportados en T. infestans pero no en los otros triatominos analizados (Juárez y Blomquist 1993, Juárez et al. 2001). Dentro de los monometilalcanos podemos mencionar como ejemplos las series con ramificación en posición 4- presentes en cadenas pares de 24 a 36 carbonos y la profusa serie de isómeros terminales 3metil tanto en cadenas pares como impares mientras que en los dimetilalcanos, las series de 3,x- y 4,x- en cadenas impares de 25 a 39 carbonos y pares de 34 a 38 respectivamente así como la presencia de isomeros $15, \mathrm{x}$ - en cadenas de 33 a 39 carbonos.

Sin embargo, las especies que componen el subcomplejo $T$. sordida presentan además, una serie de estructuras que las distinguen de las especies analizadas con anterioridad ( $T$. infestans, T. mazzottii, R. prolixus), como los 6-monometilalcanos en cadenas pares, los dimetilalcanos con ramificaciones en posiciones 3,15- o 3,17- en cadenas impares o 6,x- y 14,18- en pares y los trimetilalcanos 7,13,17- en cadenas de 29 a 31 carbonos (exclusivamente en $T$. garciabesi) y $14,18, \mathrm{x}-$ en cadenas pares de alto peso molecular. Estas diferencias podrían estar relacionadas a modificaciones en las ácido graso sintetasas microsomales, responsables de la inserción de grupos metilo en puntos específicos de las cadenas hidrocarbonadas en formación.

\section{2. Taxonomía del subcomplejo Triatoma sordida.}

El subcomplejo T. sordida es un grupo de especies de importancia epidemiológica, ya que éstas se encuentran naturalmente infectadas con $T$. cruzi, tienen diversos grados de invasión del ambiente humano (hábitats doméstico y peridoméstico) y, como se vio en el caso de $T$. sordida, colonizan las viviendas actuando como vectores primarios tras la eliminación de T. infestans (Dias 1988).

Las relaciones taxonómicas de las especies que integran el subcomplejo T. sordida han sido estudiadas en diversas oportunidades, mediante caracteres morfológicos, bioquímicos, 
morfométricos y genéticos. En la revisión de los triatominos que llevaron a cabo Lent y Wygodzinsky (1979) ellos distinguieron, tras el estudio de diversas características morfológicas, la existencia de tres especies reconocibles ( $T$. sordida, T. guasayana y $T$. patagonica). En cambio, sinonimizaron a T. garciabesi con T. sordida, basados en la gran variabilidad de los caracteres morfológicos y cromáticos utilizados para diagnosticar a la especie; considerando entónces a $T$. garciabesi como una población de $T$. sordida adaptada a ambientes más secos.

Gorla et al. (1993) estudiaron la sistemática de T. sordida, T. guasayana y $T$. patagonica mediante el análisis de 17 variables morfométricas y caracteres de la genitalia del macho. En sus resultados T. sordida se diferencia completamente de las otras dos especies, sin embargo el análisis no logra distinguir completamente a $T$. guasayana de $T$. patagonica. Los autores en base a sus resultados, la alta capacidad de dispersión de T. sordida, la gran variabilidad morfológica y grados de superposición de las distribuciones geográficas, plantean un hipótesis de especiación en un gradiente norte-sur con T. sordida como la especie mas antigua (originada en el sur de Brasil), y T. patagonica como la derivada más recientemente.

García et al (1995), utilizaron el patrón de corrida electroforética de isoenzimas torácicas y gonadales para estudiar las relaciones entre cuatro especies de triatominos, entre las cuáles se encontraban $T$. sordida y $T$. guasayana. Sus resultados validaron los presentados por Gorla et al. 1993 en cuanto a que estas son especies distintas.

Panzera et al. (1997), a partir de análisis citogenéticos e isoenzimáticos, estudiaron distintas poblaciones de T. sordida provenientes de Argentina y Brasil, así como las relaciones con $T$. garciabesi, T. guasayana y T. patagonica. Como resultado de los análisis se encontró que T. sordida se diferenciaba en dos grupos netamente separados, uno integrado por las poblaciones de Argentina y el otro por las de Brasil mientras que T. garciabesi no podía distinguirse como especie distinta, quedando incluida en el grupo que comprendía las poblaciones argentinas. T. guasayana y T. patagonica conservaron su estatus de especie, quedando relacionadas entre si y a su vez claramente diferenciadas de $T$. sordida. Respecto a la diferenciación de $T$. sordida en dos grupos, los autores especulan que pueden tratarse de dos poblaciones genéticamente diferenciadas o de dos especies distintas, apoyando esta última hipótesis, y sugieren la realización de cruzamientos experimentales a fin de corroborar la viabilidad de los híbridos.

Noireau et al. (1998), estudiaron la variabilidad en 12 isoenzimas torácicas de especímenes de T. sordida y T. guasayana colectados en las regiones andina y chaqueña de Bolivia. Como resultado de los análisis, los autores revalidaron la separación en especies de $T$. 
sordida y $T$. guasayana no hallando híbridos naturales en las zonas de superposición de sus distribuciones geográficas. Además encontraron que los especímenes de $T$. sordida se segregan en dos grupos diferenciados a los cuales llamaron "Grupo 1" y "Grupo 2". Ejemplares de estos dos grupos coexistían en simpatría en la región boliviano-chaqueña de Izozog y Tita. Se postuló además la existencia de híbridos putativos de estos dos grupos basados en el perfil de electroforesis de ciertos especímenes colectados en esa zona.

Jurberg et al. (1998), a partir del estudio de la morfología de la cabeza y la genitalia, así como mediante análisis isoenzimáticos y citogenéticos, revalidaron a T. garciabesi como especie distinta de T. sordida.

Todos estos resultados, han sido convalidados en distintas publicaciones mediante el empleo de técnicas citogenéticas (Hornos et al. 1996, Rebagliati et al. 1998), isoenzimáticas (Noireau et al. 1999a, b) y moleculares (Sainz et al. 2004).

La separación en especies dentro de este subcomplejo ha planteado dudas considerando el punto de vista clásico (Mayr 1970), dado que los cruces experimentales han dado en la mayoría de los casos una completa interfertilidad (T. guasayana x $T$. sordida en el Instituto Oswaldo Cruz, no publicado, Pietrokovsky et al. 1994; T. garciabesi x T. sordida, Usinger et al. 1966). Sin embargo, se han establecido otros puntos de vista sobre la delimitación de las especies, donde éstas son definidas en base a la evaluación numérica de datos morfológicos, bioquímicos, moleculares etc., más que en consideraciones sobre su aislamiento reproductivo (Sokal 1973). Este concepto ha sido aplicado a los resultados e inferencias surgidos de esta tesis.

El análisis basado en el patrón de HC cuticulares revela que dentro del subcomplejo $T$. sordida pueden reconocerse tres especies: T. sordida, T. garciabesi y T. guasayana, las cuales evidencian una alta similitud entre ellas. Teniendo en cuenta la cantidad de ejemplares y localidades estudiadas, nuestro análisis es el más exhaustivo en cuanto a las distribuciones geográficas de las especies consideradas.

T. guasayana es la especie que presenta la mayor diferenciación del subcomplejo. Esta diferenciación está basada principalmente en la ausencia de varios HCs ramificados presentes en las otras especies, así como en la mayor abundancia de los isómeros trimetil internos del C37 y de los distintos 5,x-dimetil isómeros del C39, presentes a nivel de trazas en las otras. Las distintas poblaciones muestran un bajo grado de diferenciación entre ellas, que se corresponde con su proximidad geográfica. Los especímenes de Mataral son los más diferenciados de la especie en base principalmente a la abundancia de los distintos isómeros de monometilalcanos internos del C37. Esta distinción de base bioquímica se corresponde con 
las importantes diferencias morfológicas observadas en esta población en cuanto al ancho del abdomen y otros caracteres (Macedo Lopes, comunicación personal).

La presencia de una serie de $\mathrm{HC}$ exclusivos como el 7,13,17-trimetil C29, C30 y C31 permiten la diagnosis de $T$. garciabesi como especie distinta de las otras, sumado a la mayor abundancia del 3-metil C27. La falta de eficiencia en la diferenciación entre las localidades analizadas, pese al empleo de $10 \mathrm{HC}$ en la discriminación, sugiere que esta especie es genéticamente muy homogénea.

Nuestro análisis sobre $T$. sordida coincide con y confirma los resultados de la aplicación de otros marcadores, en cuanto a que la especie se encuentra dividida genéticamente en dos grupos los cuales podrían ser considerados como subespecies. El HC que constituye el marcador principal de esta diferenciación es el 3-metil C31, que presenta una abundancia relativa de entre 2 a 10 veces mayor en los especímenes del grupo 2 respecto a los del grupo 1. De acuerdo al análisis de hidrocarburos, que es el más abarcativo hecho hasta ahora dentro de la distribución geográfica de la especie, estas subespecies presentan una distribución geográfica que se corresponde con un sentido noreste-suroeste y en donde se pueden encontrar zonas de simpatría, como la de Izozog en Bolivia. La existencia de dos grupos netamente definidos dentro $T$. sordida fue evidenciada en los análisis que Panzera et al. (1997) hiciera de poblaciones argentinas y brasileñas, mientras que las regiones de superposición en las distribuciones geográficas de estos grupos, fueron detectadas en los análisis de Noireau et al. (1998), así como fueron detectados a partir de estos análisis la existencia de posibles insectos híbridos; nuestros resultados en conjunto con éstos mencionados avalan la consideración de estos grupos como subespecies. Asimismo, su distribución sugiere una adaptación diferencial a los hábitats, encontrándose al grupo 2 en las regiones secas y al grupo 1 en las húmedas. Los insectos de la región andina de Quillacollo presentan el patrón de $\mathrm{HC}$ más atípico de la especie; además de la alta proporción del 3-metil C31 (13\%), se puede observar en la tabla 8 que ningún $\mathrm{HC}$ contribuye especialmente a la separación de esos especímenes en la FD2, y esto es debido a que en realidad están todos contribuyendo casi por igual. Esta población presenta asimismo, una alta proporción de HCs metil ramificados de larga cadena sin embargo, el bajo número de ejemplares analizados impide hacer especulaciones consistentes respecto a ésta.

En el análisis de los ejemplares de T. patagonica, no se pudieron encontrar HC diagnósticos que permitieran diferenciarla como especie, por lo cuál éstos quedan incluidos en nuestros análisis como una población más de T. guasayana. El hecho de que estos insectos, de distribución exclusiva en Argentina, se relacionen más estrechamente con ejemplares de $T$. 
guasayana colectados en Santiago del Estero, lleva a sugerir que este grupo se ha diferenciado a partir de ejemplares argentinos de $T$. guasayana, ocupando distintos ambientes y regiones geográficas, pero que aún no se habría diferenciado lo suficiente como para ser considerado otra especie.

La mayor variabilidad y estructuración poblacional exhibida por T. sordida podrían indicar que ésta es la especie más antigua dentro del subcomplejo. Esta hipótesis encuentra refuerzo en la reversión que muestran los insectos cruza entre $T$. guasayana y $T$. sordida hacia un patrón de hidrocarburos similar al grupo 1 de T. sordida.

V. 3. Análisis de la variabilidad intraespecífica en $T$. dimidiata mediante el patrón de hidrocarburos cuticulares

T. dimidiata es una especie que plantea un serio problema en el ámbito del control de la enfermedad de Chagas, y esta dificultad esta indudablemente ligada a su alta variabilidad genética. La variabilidad intraespecífica más alta encontrada en los triatominos hasta el momento en relación a las diferencias en las secuencias del ITS-2 del rDNA, correspondía a T. infestans con un valor de $2.7 \%$ en todo su rango de distribución geográfica (Bargues et al. 2006); sin embargo el valor de $10.2 \%$ encontrado en $T$. dimidiata excede ampliamente el valor máximo para la variabilidad intraespecífica en el género Triatoma sp. (Bargues et al. 2008). Esta especie no muestra una preferencia definida por factores orográficos o climáticos específicos pudiendo encontrarse en diversos ecosistemas; en bosques húmedos y cálidos como en regiones mas secas y de clima templado a altitudes que oscilan entre los 0 a los 2000 m.s.n.m. y (Zeledón et al. 2001, Dumonteil et al. 2002, Monroy et al. 2003b). A su amplia distribución geográfica se le suma una notable adaptación a colonizar diversos ámbitos dentro del ambiente silvestre, peridoméstico y doméstico (Schofield 2002). Se ha establecido la existencia de un flujo de insectos entre los ambientes silvestres y domésticos que puede estar condicionado por la geografía de la región; en estudios hechos en Guatemala no se ha encontrado intercambio (Calderón et al. 2004), mientras que los mismos análisis efectuados en Colombia establecieron un movimiento contínuo de insectos (Ramírez et al. 2005). Sin embargo en distintas localidades de Guatemala los reportes de reinfestación de las zonas tratadas con insecticidas son variables, con situaciones de ausencia de reinfestación como en el estado de Zacapa (Nakagawa et al. 2003a) a otras como las del estado de Jutiapa, donde en menos de 6 meses se volvieron a encontrar insectos en las viviendas (Nakagawa et al. 2003b). Reinfestaciones rápidas han sido reportadas además en el estado mexicano de Yucatán (Dumonteil et al. 2004). 
En pocos trabajos se ha estudiado detalladamente la variabilidad de una especie mediante la caracterización de los hidrocarburos cuticulares de distintas poblaciones a lo largo de su distribución geográfica. Haverty et al. (1988) estudiaron el género de termitas Zootermopsis sp. Emerson 1933 distribuidas en la costa occidental de América del Norte. A partir del análisis de distintas colonias se encontraron cuatro fenotipos de hidrocarburos que se correspondían con bastante precisión con las distintas especies del género, determinadas mediante caracteres morfológicos. Asimismo, dentro de cada fenotipo, el patrón de hidrocarburos permitió reconocer las distintas castas que componen una colonia. Brown et al. (1997), analizando el perfil de hidrocarburos de 24 poblaciones de la cucaracha australiana Geoscapheus dilatatus Saussure 1864, encontraron dos fenotipos caracterizados por la presencia o ausencia de alquenos. Las cucarachas de cada fenotipo presentaban además diferencias morfológicas y distinta distribución geográfica, lo cual llevó a los autores a sugerir la necesidad de reconsiderar la existencia de dos especies distintas. La variabilidad de hidrocarburos era distinta dentro de cada fenotipo, además de haberse encontrado tres poblaciones que no pertenecían a ninguno de ellos.

T. dimidiata es una especie de amplia distribución y que presenta, a lo largo de ella, una gran variabilidad en su morfología y patrones de coloración (Lent y Jurberg 1985). Esta variabilidad ha llevado a diversos autores que la han estudiado a distinguir especies y subespecies.

Usinger (1944), basándose en la forma de la cabeza y las relaciones entre su largo y el ancho medido a la altura de los ojos, distingue tres subespecies: $T$. $d$. maculipennis que incluye la mayor parte de los ejemplares mexicanos, $T$. $d$. capitata que comprende a los ejemplares colombianos y $T$. $d$. dimidiata que agrupa al resto de especímenes mexicanos y a los ejemplares centroamericanos. Caracterizó a la subespecie $T$. d. capitata por una cabeza elongada y estrecha con ojos más pequeños en relación a $T$. $d$. maculipennis.

En la revisión de los triatominos llevada a cabo por Lent y Wygodzinsky en 1979 y tras el análisis de 160 especímenes de todo el rango de distribución, los autores concluyen que la gran variabilidad intrínseca de la especie, de naturaleza clinal, no permite su segregación en distintas subespecies, atribuyendo dicha variabilidad a su alta labilidad genética. Los autores además reafirman este concepto tras el estudio de ejemplares colectados en dos sistemas de cuevas distintos, los de Lanquín en Guatemala y los de Río Frío en Bélice, pero donde los especímenes comparten varios rasgos morfológicos derivados de la adaptación a la vida en cuevas como ser falta de pigmentación general, ojos y ocelos reducidos. 
Marcilla et al. (2001) estudiaron las relaciones filogenéticas de varias especies de triatominos mediante las diferencias en la secuencia del ITS-2 del rDNA, incluyendo varias poblaciones de $T$. dimidiata de Mexico, Honduras, Ecuador y Nicaragua. En este análisis los insectos de Yucatán (Mexico) se diferencian claramente del resto de los ejemplares, a un nivel considerado por los autores como de una especie distinta. El resto de las poblaciones mostró una variación clinal en sentido norte-sur aunque la estrecha relación de los ejemplares de Ecuador con los de América Central sugería que éstos fueron introducidos de manera directa por el hombre.

Dorn et al. (2003) analizaron mediante RAPD la variabilidad genética y el flujo entre especímenes de villas mas o menos cercanas del sur de Guatemala. Sus resultados muestran una alta variabilidad genética con alto porcentaje de polimorfismo en los loci utilizados y un elevado valor de heterocigosis; además los valores obtenidos de distancia génica entre poblaciones mostró un activo flujo entre ellas.

Bustamante et al. (2004) estudiaron la variabilidad de ocho poblaciones de $T$. dimidiata colectadas en localidades de Guatemala, Colombia, Mexico y Honduras; y asimismo evaluaron su relación con otras especies de triatominos de la región. Para ello emplearon métodos de morfometría tradicional y geométrica aplicados a distintas medidas de la cabeza de los insectos. Sus resultados muestran una similitud entre las poblaciones relacionada a su proximidad geográfica excepto para la población de Petén (Guatemala) que se relaciona mas estrechamente con la de Veracruz (Mexico) y las de Lanquín (Guatemala) y Boavita (Colombia) que se diferencian netamente del resto, a un nivel comparable al de distintas especies. En la comparación interespecífica, $T$. dimidiata se relaciona más estrechamente con las otras epecies analizadas del complejo T. phyllosoma.

Calderón et al. (2004) evaluaron mediante la técnica de RAPD la variabilidad y flujo genéticos, así como las relaciones de similitud entre tres poblaciones del norte de Guatemala (incluyendo Petén y las cuevas de Lanquín) y tres del sur (incluyendo Jutiapa). Obtuvieron altos valores de heterocigosis así como de polimorfismo en los loci usados, indicativos de una alta variabilidad genética. Los insectos de Petén y Lanquín exhibieron los grados más altos de diferenciación mientras que las poblaciones del sur de Guatemala se relacionaban en base a su vecindad geográfica.

Catala et al. (2005) analizaron el patrón de sensilas antenales de diversas poblaciones de T. dimidiata originarias de Mexico (Veracruz, Hidalgo, Yucatán), Guatemala (Petén, Lanquín, Jutiapa, Santa Rosa), Honduras, Costa Rica (Heredia, San Jose) y Colombia (Santander). Encontraron similitudes de abundancia de sensilas específicas en los insectos 
colectados en domicilios y en cuevas, en relación a la estabilidad de ambos hábitats y en oposición al ambiente silvestre. Al analizar los insectos colectados en un mismo ambiente obtuvieron un patrón de similitudes entre especímenes relacionado a su proximidad geográfica.

Panzera et al. (2006) analizaron el contenido de DNA, la forma y la estructura de los cromosomas meióticos en machos y hembras de $T$. dimidiata de diversas localidades de la costa atlántica y Yucatán en Mexico, Petén y la costa pacífica en Guatemala, la costa pacífica de El Salvador y el norte de Colombia. Estos estudios muestran que la especie esta dividida en tres citotipos: el citotipo 1, distribuido a lo largo del este de Mexico, la costa pacífica de Guatemala y El Salvador y en Colombia con capacidad de habitar tanto ambientes silvestres como domésticos y con activo flujo genéticos entre poblaciones; el citotipo 2, ubicado en Yucatán y el citotipo 3, encontrado en la región selvática de Petén en Guatemala, estos dos últimos de habitat fundamentalmente silvestre y con escaso o nulo flujo entre poblaciones. Los autores especulan que los tres citotipos implican la existencia de especies crípticas derivadas a partir de una especie antecesora de amplia distribución.

Lehmann et al. (2005) estudiaron las relaciones entre especimenes colectados en la costa este y Yucatán en Mexico, y Petén en Guatemala. Para ello emplearon técnicas de morfometría utilizando medidas de la cabeza y alas de los insectos sobre las que aplican análisis discriminante. Detectaron dos grupos claramente diferenciados, uno formado por los ejemplares de la costa este de Mexico y otro que reune a los insectos de Yucatán y Petén.

Ramirez et al (2005) analizaron las relaciones entre poblaciones domésticas, peridomésticas y silvestres de $T$. dimidiata en una zona de Boyaca en Colombia mediante la técnica de RAPD. Establecieron que existe un flujo sostenido de insectos entre los distintos ambientes por lo que los especímenes silvestres constituyen un riesgo epidemiológico.

Bargues et al. (2008) analizaron la variabilidad intraespecífica de T. dimidiata y su relación con diversas especies norteamericanas, centroamericanas y sudamericanas del género Triatoma sp. empleando las variaciones en la secuencia del ITS-2 del rDNA; el estudio contempló numerosas localidades de Mexico, Guatemala, Honduras, Nicaragua, Panamá, Colombia y Ecuador. El análisis sobre $T$. dimidiata resultó en una división de la especie en tres grupos: El grupo 1, dividido a su vez en dos subgrupos, el A que comprende la mayor parte de los insectos de Guatemala, Honduras, Nicaragua y Ecuador, y el B que agrupa la mayoría de los haplotipos hallados en Colombia y Panamá; el grupo 2 que comprende los haplotipos de la mayor parte de Mexico y el grupo 3 que nuclea específicamente a los especímenes mexicanos de Yucatán e isla de Cozumél, los de Petén en el norte selvático de 
Guatemala y un par de haplotipos colectados en Honduras. La buena correlación entre estos grupos, con la distintas subespecies descriptas por Usinger en 1944, llevó a los autores a revalidar esta propuesta clasificatoria, desestimando la unificación en una única especie realizada por Lent y Wygodzinsky (1979).

El análisis del patrón de hidrocarburos demuestra que esta especie presenta una alta variabilidad tanto intra como interpoblacional. La alta heterogeneidad de estos insectos se puede inferir a partir de los valores de error estándar de las abundancias relativas de los hidrocarburos en los distintos grupos analizados (Tabla 30)

Los dendrogramas derivados del análisis de hidrocarburos muestran patrones de diferenciación que se pueden correlacionar claramente con un nivel de aislamiento genético dado por características geográficas y/o ambientales. En regiones geográficamente cercanas y ambientalmente similares, como los departamentos de Boyaca y Casanare en Colombia, los departamentos de Guanacaste y Heredia o Cartago y San José en Costa Rica y los departamentos de Hidalgo y Veracruz o Yucatán y la cercana isla de Cozumél en Mexico, la baja distancia de Mahalanobis muestra que existe o ha existido recientemente un intercambio de insectos frecuente entre las localidades (figuras 55 y 57); lo cual coincide con los estudios que señalan la existencia de metapoblaciones en estas regiones (Dorn et al. 2003, Lehmann et al. 2005). En cambio, los insectos colectados en el interior de las cuevas de Lanquín (Guatemala) y de Bélice, exhiben respecto al perfil de hidrocarburos típico de la especie, un grado de diferenciación comparable al de otras especies. Ambos grupos muestran una notable escasez en la abundacia relativa del $n$-C27 sumado a una alta proporción de hidrocarburos ramificados de alto peso molecular. La base genética de esta diferenciación, podría estar relacionada a una expresión o regulación diferenciales de las enzimas involucradas en el proceso de elonganción de cadenas metil ramificadas, y de liberación a partir del sistema elongante de las cadenas de ácidos grasos lineales de 28 átomos de carbono, precursoras del $n$-C27. Es evidente que en estos insectos han ido evolucionando su perfil de hidrocarburos, coloración, tamaño de los ojos y demás caracteristicas fenotípicas en el sentido de su adapatación a vivir en el interior de las cuevas donde tienen refugio y alimentación aseguradas a partir de los murciélagos que cohabitan con ellos; este perfil de hidrocarburos con predominio de cadenas ramificadas implicaría una adaptación que coincide con lo descripto para un ambiente protegido, de alta humedad (Woodrow et al. 2000). Desde el estudio llevado a cabo por Lent y Wygodzinsky (1979) estos especímenes no han sido analizados en profundidad, pero los análisis morfométricos de Bustamante et al. (2004), los de RAPD de Calderón et al. (2004) y el reciente estudio sobre las secuencias del ITS-2 (Bargues 
et al. 2008) coinciden con nuestras conclusiones sobre la gran diferenciación fenotípica y genotípica de estos insectos, y de la necesidad de reconsiderar su estatus específico. La realización de cruces experimentales podría aportar pruebas sobre su capacidad de cruzamiento y la aptitud reproductiva de la descendencia, de haberla.

Excluyendo los insectos de las cuevas de Lanquín y Bélice, la mayor parte de las poblaciones analizadas se distribuye en tres grandes agrupamientos, netamente diferenciables (figura 58)

Figura 58. Dendrograma de la figura 57 detallando los agrupamientos principales de la especie derivados a partir del análisis de hidrocarburos cuticulares.

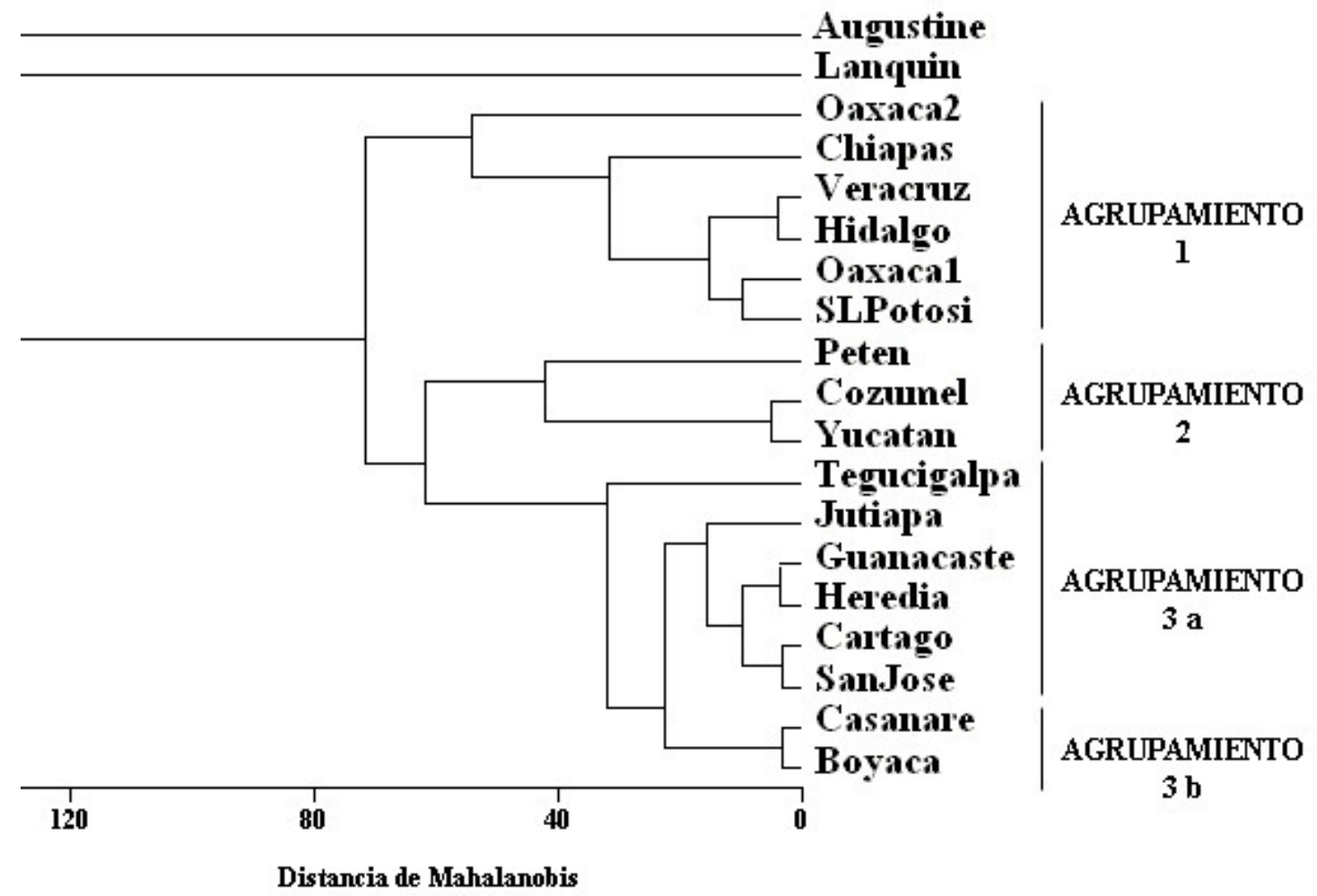

Agrupamiento 1: El conformado por todas las poblaciones mexicanas exceptuando los insectos de Yucatán y Cozumél. Este agrupamiento muestra una similitud entre los insectos relacionada a su proximidad geográfica y una variación que sigue un gradiente clinal en sentido norte-sur con los especímenes de Chiapas como los más diferenciables dentro de este gradiente. Asimismo se puede destacar que la mayor disimilitud es exhibida por los insectos colectados en la región de la costa pacífica del estado de Oaxaca, aislada geográficamente del 
resto del estado y del país por la elevaciones montañosas de las Sierras Madres del Sur; este grupo designado como Oaxaca 2 muestra un patrón de hidrocarburos claramente distinto a los insectos colectados en la región oriental de las Sierras Madres (especímenes agrupados dentro del nombre Oaxaca 1), caracterizado por la menor abundancia de los HC lineales $n$-C31 y $n$ C33 pero la mayor proporción de los HC ramificados con KI 3755 y 3779. Esta diferenciación del grupo Oaxaca 2 esta muy posiblemente relacionada al aislamiento geográfico (y por ende genético) producido por las Sierras Madres del Sur lo que ha llevado a esta población a evolucionar independientemente del resto de los ejemplares de la región central y sur de Mexico. Este agrupamiento obtenido del análisis de hidrocarburos coincide con la subespecie T. dimidiata maculipennis definida por Usinger (1944), con parte del citotipo 1 identificado en estudios cromosómicos por Panzera et al. (2006) y con el grupo 2 y algunos haplotipos del subgrupo 1A del ITS-2 (Bargues et al. 2008).

Agrupamiento 2: El conformado por los insectos de Yucatán e isla de Cozumél en Mexico y Petén en el norte de Guatemala, geográficamente cercanos. La relación entre los especímenes de Petén y los de la península de Yucatán, esta basada principalmente en la inclusión en el análisis del HC con KI 2932, por lo que no es muy consistente; en otros resultados de DA (figura 55) y en el PCA (figura 53) los insectos de Petén tienden a agruparse diferenciándose de todas las poblaciones, aunque no a un nivel tan marcado como los insectos de Bélice y Lanquín. Este agrupamiento coincide con el citotipo 3 hallando en Petén y el citotipo 2 de Yucatán en los estudios cromosómicos de Panzera et al. (2006). Respecto al análisis de ITS-2 realizado por Bargues et al. (2008) es interesante notar que de los haplotipos encontrados en la especie, las tres localidades comparten el H28 y de hecho este es el único encontrado en la isla de Cozumél; además los insectos de Petén y Yucatán comparten el haplotipo H25 en conjunto a los propios de cada región (H30 en Petén; H26, H27 y H31 en Yucatán). Es muy problable que la combinación en nuestra muestra de varios haplotipos, tanto propios como compartidos, justifique en los insectos de Petén, la tendencia ambigüa de agruparse tanto separadamente como en relación a los ejemplares mexicanos de la península de Yucatán. Análisis morfométricos mostraron una tendencia de los insectos de Petén a agruparse con distintas poblaciones, lo que llevó a sugerír la existencia de varios genotipos (Bustamante et al. 2004, Lehmann et al. 2005).

Agrupamiento 3: El integrado por los ejemplares colectados en países de América Central y del Sur. Las relaciones de semejanza entre las distintas poblaciones se basan también en su proximidad geográfica, de tal manera que se forma un grupo de los insectos centroamericanos (Agrupamiento 3 a) y otro que agrupa a las dos poblaciones de Colombia en Sudamérica 
(Agrupamiento $3 \mathrm{~b}$ ). Este agrupamiento con sus dos subdivisiones comprende a las subespecies $T$. $d$. dimidiata (Centroamérica) y $T$. $d$. capitata (Colombia) definidas por Usinger (1944) y al Subgrupo 1A y 1B respectivamente en la caracterización de las secuencias de ITS2 realizada por Bargues et al. (2008). Se puede inferir a partir de las distancias de Mahalanobis derivadas del DA que existe un mantenido aislamiento genético entre los países de América Central analizados, así como también respecto a Colombia.

Nuestros resultados, en mayor o menor consenso con los obtenidos a partir de otras técnicas moleculares (Marcilla et al. 2001, Bargues et al. 2008), citogenéticas (Panzera et al. 2006) y morfométricas (Catalá et al. 2005), no coinciden con la idea establecida por Lent y Wygodzinsky (1979), en cuanto a la variación de naturaleza clinal de una única especie, sino que muestran conjuntos de poblaciones netamente definidos, aislados genéticamente unos de otros y en estrecha relación a la división en subespecies sugerida por Usinger (1944) (figura 59).

Figura 59. Mapas que muestran el resultado del análisis de $\mathrm{HC}(\mathrm{C})$ comparado con los obtenidos a partir de los análisis citogenético (A) y del ITS-2 rDNA (B), en diversas poblaciones de Triatoma dimidiata.
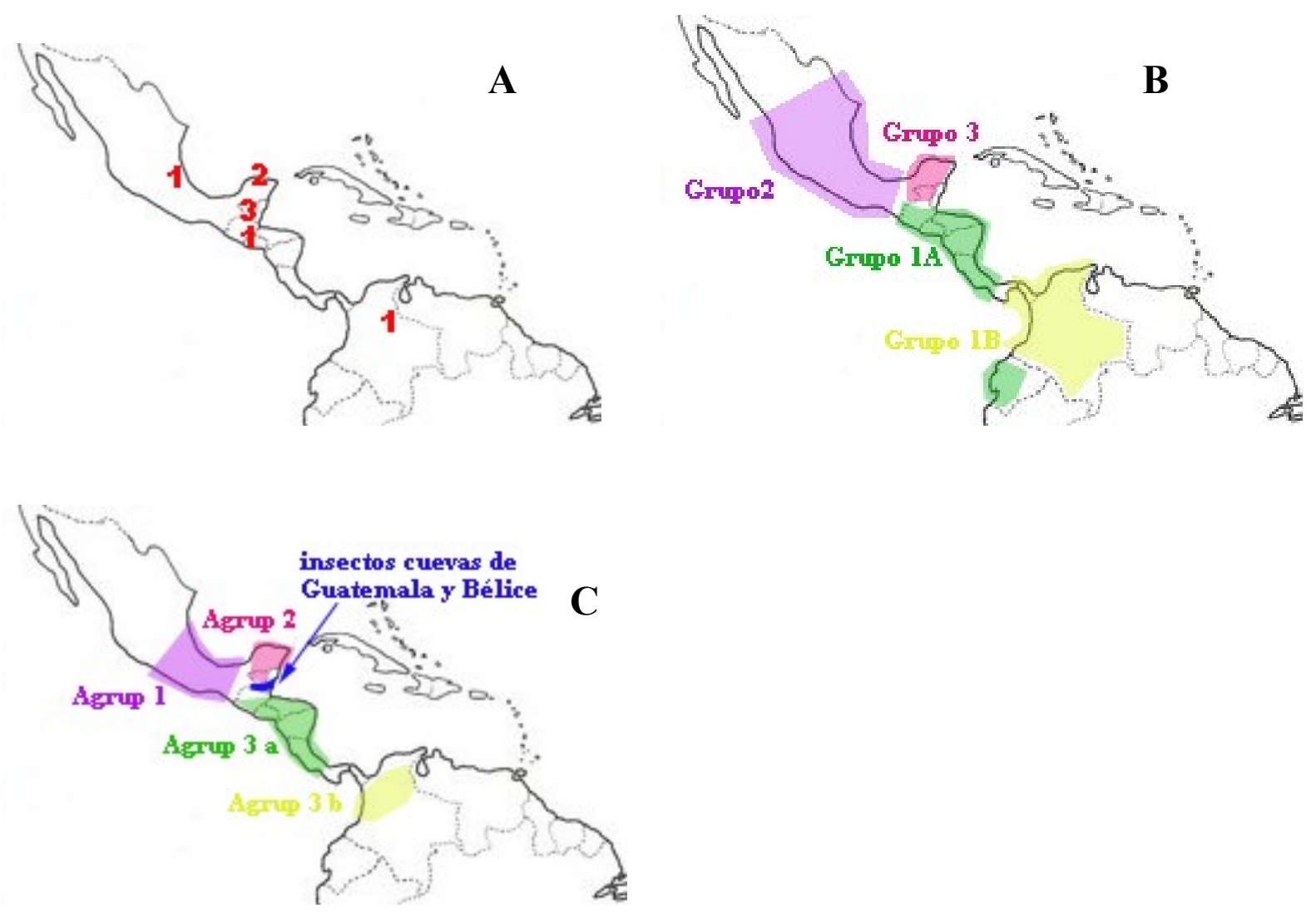

A) 1,2 y 3: citotipos 1, 2 y 3 respectivamente, según Panzera et al. 2006; B) según Bargues et al. 2008. 
De esta manera el Agrupamiento 1 se corresponde con la subespecie T. dimidiata maculipennis y el Agrupamiento 3 con sus dos subdivisiones principales comprende a las subespecies $T$. $d$. dimidiata en Centroamérica y $T$. $d$. capitata en Colombia, con una relación evolutiva mucho más cercana entre estas dos últimas, que con respecto a $T$. d. maculipennis. En relación a los insectos del Agrupamiento 2, nuestros análisis llevan a considerarlos como otra subespecie además de las tres ya definidas por Usinger (1944), sin embargo, las relaciones de similitud entre los insectos de Petén y las otras dos poblaciones no son muy consistentes; en los análisis citogenéticos éstos se diferencian como otro citotipo (Panzera et al. 2006), y en los análisis morfométricos tienen tendencia a relacionarse tanto con los ejemplares mexicanos de Yucatán (Lehmann et al. 2005) como con los de Veracruz (Bustamante et al. 2004). De aceptarse la presencia de distintas subespecies, la delimitación precisa de las zonas de distribución geográfica y su superposición, podría esclarecer el comportamiento ambigüo de los insectos de Petén respecto a sus relaciones de similitud. Serían necesarios ulteriores análisis del patrón de hidrocarburos mediante CGC-MS para dilucidar las relaciones de los especímenes de Petén con los de Yucatán y Cozumél en Mexico así como para establecer de manera más precisa la magnitud de diferenciación de insectos como los de las cuevas de Lanquín y Bélice, y los de la costa pacífica del estado mexicano de Oaxaca (Oaxaca 2), los cuáles en virtud de las características geográficas y ambientales de sus hábitats, parecen estar experimentando una gran divergencia evolutiva.

\section{4. Conclusiones finales.}

Los resultados obtenidos tanto al analizar el subcomplejo Triatoma sordida como a Triatoma dimidiata, demuestran que el patrón de hidrocarburos cuticulares constituye un marcador taxonómico útil para establecer la variabilidad genética intraespecífica así como las relaciones entre especies de triatominos. Las principales ventajas de su utilización radican tanto en la sencillez y accesibilidad de los procedimientos de obtención y análisis, como en la sensibilidad para detectar variaciones a nivel poblacional, interespecífico e intergenérico entre otros.

La información aportada por éste y otros marcadores podría ser de gran utilidad para los programas de control de la enfermedad al momento de diseñar estrategias específicas contra los grupos obtenidos tras los análisis, evaluar los períodos de reinfestación de los sitios tratados a partir de los focos silvestres, discernir las vías de migración de las distintas especies de triatominos y delimitar con precisión sus áreas de distribución, entre otras ventajas. La aparición en la actualidad en distintas localidades de Argentina y Bolivia, de poblaciones de 
T. infestans altamente resistentes a insecticidas piretroides (Picollo et al. 2005), y su correlación con agrupaciones fenotípicas determinadas mediante análisis de hidrocarburos (Juárez et al., no publicado) y otros estudios, plantea la vigencia y la necesidad del empleo de estos marcadores. 


\section{Referencias bibliográficas}

Acosta N., López E.,González N., Fernández M.J., Rojas de Arias A. 2002. Isoenzyme profiles of Triatoma infestans Populations from Eastern and Western Regions of Paraguay. Memorias del Instituto de Investigaciones en Ciencias de la Salud, Universidad Nacional de Asunción. Vol.1 No 1 .

Adams D.C., Funk D.J. 1997. Morphometric inferences on sibling species and sexual dimorphism in Neochlamisus bebbianae leaf beatles: multivariate applications of the thinplate spline. Syst. Biol. 46: 180-194.

Altube H.A., Ontivero Urquiza M., Rivata R.S., Taborda R.J. 2001. Utilizacion de isoenzimas de extractos de hojas en la caracterización de cultivares de duraznero (Prunus persica (L) Batsch). Rev. Bras. Frutic. 23(2): 345-349.

Armold M.T., Blomquist G.J., Jackson L.L. 1969. Cuticular lipids of insects. III. The surface lipids of the aquatic and terrestrial life forms of the big stonefly Pteronarcys californica Newport. Comp. Biochem. Physiol. 31: 685-692.

Bargues M.D., Marcilla A., Ramsey J.M., Dujardin J.P., Schofield C.J., Mas-Coma S. 2000. Nuclear rDNA-based molecular clock of the evolution of Triatominae (Hemiptera: reduviidae), vectors of Chagas disease. Mem. Inst. Oswaldo Cruz :95(4): 567-573.

Bargues M.D., Klisiowicz D.R., Panzera F., Noireau F., Marcilla A., Perez R., Rojas M.G., O’Connor J.E., Gonzalez-Candelas F., Galvão C., Jurberg J., Carcavallo R.U., Dujardin J.P., Mas-Coma S. 2006. Origin and phylogeography of the Chagas disease main vector Triatoma infestans based on nuclear rDNA sequences and genome size. Inf Gen Evol 6: 46-62.

Bargues M.D., Klisiowicz D.R., Gonzalez-Candelas F., Ramsey J.M., Monroy C., Ponce C., Salazar-Schettino P.M., Panzera F., Abad-Franch F., Sousa O.E., Schofield C.J., Dujardin J.P., Guhl F., Mas-Coma S. 2008. Phylogeography and Genetic Variation of Triatoma dimidiata, the Main Chagas Disease Vector in Central America, and Its Position within the Genus Triatoma. PLoS Negl. Trop. Dis. 2(5): e233. 
Bartelt R.J., Krick T.P., Jones R.L. 1984. Cuticular hydrocarbons of the yellowheaded spruce sawfly Pikonema alaskensis. Insect Biochem. 14: 209-213.

Barthlott W., Theisen I., Borsch T., Neinhuis C. 2003. Epicuticular waxes and vascular plant systematics: integrating micromorphological and chemical data. En: Deep Morphology: Toward a Renaissance of Morphology in Plant Systematics, editado por Stuessy T.F., Mayer V. y Hörandl E., Regnum Vegetabile, vol. 141. Gantner Verlag, Ruggell/Liechtenstein, pp. 189-206.

Beamont J.W.L. 1945. The cuticular lipids of insects. J. Exp. Biol. 21: 115-131.

Bellier J.P., De Wever P. 1994. Paleoenvironmental polymorphism in the Globutruncana of the aarca group (Cretaceous planktonic foraminifera): Experimental study by image analysis. Geobios 27: 285-291.

Blomquist G.J., Soliday C.L., Byers B.A., Brakke J.W., Jackson L.L. 1972. Cuticular lipids of insects: V. Cuticular wax esters of secondary alcohols from the grasshoppers Melanoplus packardii and Melanoplus sanguinipes. Lipids 7: 356-362.

Blomquist G.J., Blailock T.T., Scheetz R.W., Jackson L.L. 1976. Cuticular lipids of insects. VII. Cuticular hydrocarbons of the crickets Acheta domesticus, Gryllus pennsylvanicus and Nemobius fasciatus. Comp. Biochem. Physiol. 54B: 381-386.

Blomquist G.J., Jackson L.L. 1979. Chemistry and biochemistry of insect waxes. Prog. Lipid Res. 17: 319-345.

Blomquist G.J., Chu A.J., Remaley A.R. 1980. Biosynthesis of wax in the honeybee, Apis mellifera L. Insect Biochem 10: 313-321.

Blomquist G.J., Dillwith J.W. 1985. Cuticular lipids. En: Comprehensive Insect Physiology, Biochemistry and Pharmacology, editado por Kerkut G.A. y Gilbert L.I., $1^{\text {a }}$ edición, pag. $117-$ 154. Pergamon Press, Oxford. 
Blomquist G.J., Toolson E.C., Nelson D.R. 1985. Epicuticular hydrocarbons of Drosophila pseudoobscura (Diptera: Drosophilidae). Insect Biochem. 15: 25-34.

Blomquist G.J., Nelson D.R., de Renobales M. 1987. Chemistry, biochemistry and physiology of insect cuticular lipids. Arch. Insect Biochem. Physiol. 6: 227-265.

Blomquist G.J. 2003. Biosynthesis and ecdysteroid regulation of housefly sex pheromone production. En: Insect Pheromone Biochemistry and Molecular Biology. The Biosynthesis and Detection of Pheromones and Plan Volatiles, editado por: Blomquist G.J. y Vogt R.G. pp. 231-252. Elsevier Academic Press, London.

Brenière S.F., Taveira B., Bosseno M.F., Ordoñez R., Lozano-Kasten F., Magallón-Gastélum E., Ouaissi A., Ramsey J.M. 2003. Preliminary results of random amplification of polymorphic DNA among Triatominae of the phyllosoma complex (Hemiptera, Reduviidae). Mem. Inst. Oswaldo Cruz. 98(8): 1033-1038.

Brown W.V., Rose H.A., Lacey M.J. 1997. The Cuticular Hydrocarbons of the Soil Burrowing Cockroach Geoscapheus dilatatus (Saussure) (Blattodea: Blaberidae: Geoscapheinae) Indicate Species Dimorphism - Cuticular hydrocarbons of workers, and soldier and alate morphology. Comp. Biochem. Physiol. B 118(3): 549-562.

Brown W.V., Rose H.A., Lacey M.J., Wright K. 2000. The cuticular hydrocarbons of the giant soil-burrowing cockroach Macropanesthia rhinoceros Saussure (Blattodea: Blaberidae: Geoscapheinae): analysis with respect to age, sex and location. Comp. Biochem. Physiol. B 127: $261-277$

Bustamante D.M., Monroy C., Menes M., Rodas A., Salazar-Schettino P.M., Rojas G., Pinto N., Guhl F., Dujardin J.P. 2004. Metric variation among geographic populations of the Chagas vector Triatoma dimidiata (Hemiptera: Reduviidae: Triatominae) and related species. J. Med. Entomol. 41: 296-301.

Calderón C.I., Dorn P.L., Melgar S., Chávez J.J., Rodas A., Rosales R., Monroy C.M. 2004. A Preliminary Assessment of Genetic Differentiation of Triatoma dimidiata (Hemiptera: 
Reduviidae) in Guatemala by Random Amplification of Polymorphic DNA-Polymerase Chain Reaction. J. Med. Entomol. 41(5): 882-887.

Canale D.M., Stariolo R.L. 2002. Taller de actualización sobre vectores de la enfermedad de Chagas. Impreso por la Coordinación Nacional de Control de Vectores, Córdoba, Argentina. pp 4-6.

Capanna E., Bekele A., Capula M., Castiglia R., Civitelli M.V., Codjia J.T.C., Corti M., Fadda C. 1996. A multidisciplinary approach to the systematics of the genus Arvicanthis Lesson, 1842 (Rodentia, Murinae). Mammalia. 60: 677-696.

Carder J.H. 1989. Distinctions between celullase isoenzyme patterns of five plantpathogenic Verticillium species. Mycol. Res. 92: 297-301.

Carlson D.A., Service M.W. 1979. Differentiation between species of the Anopheles gambiae Giles complex (Diptera: Culicidae) by analysis of cuticular hydrocarbons. Ann.Trop. Med. Parasit. 73: 589-592.

Carlson D.A., Service M.W. 1980. Identification of mosquitoes of Anopheles gambiae species complex A and B by analysis of cuticular components. Science 207: 1089-1091.

Carlson D.A., Walsh J.F. 1981. Identification of two West African black flies (Diptera: Simuliidae) of the Simulium damnosum species complex by analysis of cuticular paraffins. Acta Trop. 38: 235-239.

Carlson D.A., Bolten A.B. 1984. Identification of Africanized and European honey bees, using extracted hydrocarbons. Bull. Entomol. Soc. Am. 30: 32-35.

Carlson D.A., Brenner R.J. 1988. Hydrocarbon-based Discrimination of Three North American Blattella Cockroach Species (Orthoptera: Blattellidae) Using Gas Chromatography. Ann. Entomol. Soc. Am. 81(5): 711-723. 
Carlson D.A., Milstrey S.K., Narang S.K. 1993. Classification of tsetse Glossina spp. (Diptera: Glossinidae) by gas chromatographic analysis of cuticular components. Bull. Entomol. Res. 83: 507-515.

Carlson D.A., Bernier U.R., Sutton B.D. 1998. Elution patterns from capillary GC for methylbranched alkanes. J. Chem. Ecol. 24(11): 1845-1865.

Casini C.E., Dujardin J.P., Martínez M., Pereira A.B., Salvatella R. 1995. Morphometric differentiation evidenced between two geographic populations of Triatoma infestans in Uruguay. Res. Rev. Parasitol. 55 (1): 25-30.

Catala S., Dujardin J.P. 2001. Antennal sensilla patterns indicate geographic and ecotopic variability among Triatoma infestans (Hemiptera : Reduviidae) populations. J. Med. Ent. 38(3): 423-428.

Catala S., Sachetto C., Moreno M., Rosales R., Salazar-Schettino P.M., Gorla D. 2005. Antennal phenotype of Triatoma dimidiata populations and its relationship with species of phyllosoma and protracta complexes. J. Med. Entomol. 42(5): 719-725.

Costa J., Freitas-Sibajev M.G.R., Marchon-Silva V., Quinhones Pires M., Pacheco R.S. 1997. Isoenzymes Detect Variation in Populations of Triatoma brasiliensis (Hemiptera: Reduviidae: Triatominae). Mem. Inst. Oswaldo Cruz 92(4): 459-464.

Coyne J.A. 1996. Genetics of Differences in Pheromonal Hydrocarbons Between Drosophila melanogaster and D. simulans. Genetics 143: 353-364.

Crespo R., Juárez M.P., Cafferatta L.R.F. 2000. Biochemistry of the interaction between entomopathogenous fungi and their insect host-like hydrocarbons. Mycologia 92: 528-536.

Crespo R., Juárez M.P., Dal Bello G., Padín S., Calderón Fernández G., Pedrini N. 2002. Increased mortality of Acanthoscelides obtectus by alkane-grown Beauveria bassiana. BioControl 47(6): 685-696. 
Crespo R. 2006. Bioquímica de la interacción hongo entomopatógeno-cutícula de triatomino. Tesis de la Facultad de Ciencias Naturales y Museo, UNLP

Dias J.C.P. 1988. Controle de vetores da doença de Chagas no Brasil e risco de reinvasão domiciliar por vetores secundários. Mem. Inst. Oswaldo Cruz 83 (supl. I): 387-391.

Dorn P.L., Melgar S.A., Rouzier V., Gutierrez A., Combe C., Rosales R., Rodas A., Kott S., Salvia D., Monroy C. 2003. The Chagas vector, Triatoma dimidiata (Hemiptera: Reduviidae), is panmictic within and among adjacent villages in Guatemala. J. Med. Entomol. 40: 436-440.

Dorn P.L., Monroy C., Curtis A. 2007. T. dimidiata (Latreille, 1811): A Review of its Diversity across its Geographic Range and the Relationship among Populations. Inf. Gen. Evol. 7: 343-352.

Dos Santos S.M., Lopes C.M., Dujardin J.P., Panzera F., Pérez R., Carbajal de la Fuente A.L., Pacheco R.S., Noireau F. 2007. Evolutionary relationships based on genetic and phenetic characters between Triatoma maculata, Triatoma pseudomaculata and morphologically related species (Reduviidae: Triatominae). Infect. Genet. Evol. 7(4): 469-475.

Drotz M.K., Saura A., Nilsson A.N. 2001. The species delimitation problem applied to the Agabus bipustulatus complex (Coleoptera, Dytiscidae) in north Scandinavia. Biol. J. Linnean Soc. 73: 11-22.

Dujardin J.P., Tibayrenc M., Venegas E., Maldonado L., Desjeux P., Ayala F.J. 1987. Isozyme evidence of lack of speciation between wild and domestic Triatoma infestans (Heteroptera: Reduviidae) in Bolivia. J. Med. Entomol. 24(1): 40-45.

Dujardin J.P., Forgues G., Torrez M., Martínez E., Cordoba C., Gianella A. 1998a. Morphometrics of domestic Panstrongylus rufotuberculatus in Bolivia. Ann. Trop.Med. Parasitol. 92(2): 219-228.

Dujardin J.P., Schofield C.J., Tibayrenc M. 1998b. Population structure of Andean Triatoma infestans: allozyme frequencies and their epidemiological relevance. Med Vet Entomol. 12(1): $20-29$ 
Dujardin J.P., Chavez T., Moreno J.M., Machane M., Noireau F., Schofield C.J. 1999. Comparison of Isoenzyme Electrophoresis and Morphometric Analysis for Phylogenetic Reconstruction of the Rhodniini (Hemiptera: Reduviidae: Triatominae). J. Med. Entomol. 36(6): 653-659.

Dujardin J.P., Schofield C.J., Panzera F. 2000. Les vecteurs de la maladie de Chagas: recherches taxonomiques, biologiques et ge'ne' tiques. Academie Royale des Sciences DOutre-Mer, Belgique, Brussels, Belgium.

Dumonteil E., Gourbiere S., Barrera-Perez M., Rodriguez-Felix E., Ruiz-Pina H., BanosLopez O., Ramirez-Sierra M.J., Menu F., Rabinovich J.E. 2002. Geographic distribution of Triatoma dimidiata and transmission dynamics of Trypanosoma cruzi in the Yucatan peninsula of Mexico. Am. J. Trop. Med. Hyg. 67 (2): 176-183.

Dumonteil E., Ruiz-Pina H., Rodriguez-Felix E., Barrera-Perez M., Ramirez-Sierra M.J., Rabinovich J.E., Menu F. 2004. Re-infestation of houses by Triatoma dimidiata after intradomicile insecticide application in the Yucatán peninsula, Mexico. Mem. Inst. Oswaldo Cruz. 99: $253-256$.

Etges W.J., Jackson L.L. 2001. Epicuticular Hydrocarbon Variation in Drosophila mojavensis Cluster Species. J. Chem. Ecol. 27(10): 2125-2149.

Ferrario V.F., Sforza C., Pizzini G., Vogel G., Miani A. 1993. Sexual dimorphism in the human face assessed by euclidean distance matrix analysis. J. Anat. 183: 593-600.

Foottit R.G. 1992. The use of ordination methods to resolve problems of species discrimination in the genus Cinara Curtis (Homoptera: Aphidoidea: Lachnidae). En: Ordination In The Study of Morphology, Evolution and Systematics of Insects: Applications and Quantitative Genetic Rationals; editado por Sorensen J.T. y Foottit R.G., $1^{\circ}$ edición, pag. 193-221, Elsevier, Amsterdam

Foottit R.G., Sorensen J.T. 1992. Ordination methods: Their contrast to Clustering and Cladistic techniques. En: Ordination In The Study of Morphology, Evolution and Systematics 
of Insects: Applications and Quantitative Genetic Rationals; editado por Sorensen J.T. y Foottit R.G., $1^{\circ}$ edición, pag. 1-10, Elsevier, Amsterdam.

Galvão C. 2003. A sistemática dos triatomíneos (hemiptera, reduviidae), de De Geer ao DNA. Entomol. Vect. 10(4): 511-530.

Galvão C., Carcavallo R., Rocha D.S., Jurberg J. 2003. A checklist of the current valid species of the subfamily Triatominae Jeannel, 1919 (Hemiptera, Reduviidae) and their geographical distribution, with nomenclatural and taxonomic notes. Zootaxa 202: 1-36.

García B.A., Canale D.M., Blanco A. 1995. Genetic Structure of Tour Species of Triatoma (Hemiptera: Reduviidae) from Argentina. J. Med. Entomol. 32(2): 134-137.

García B.A., Powell J.R. 1998. Phylogeny of species of Triatoma (Hemiptera: Reduviidae) based on mitochondrial DNA sequences. J. Med. Entomol. 35(3): 232-238.

Garrison R.W. 1992. Using ordination methods with geographic information: species resolution in a partially sympatric complex of neotropical Tramea dragonflies (Odonata: Libellulidae). En: Ordination In The Study of Morphology, Evolution and Systematics of Insects: Applications and Quantitative Genetic Rationals; editado por Sorensen J.T. y Foottit R.G., $1^{\circ}$ edición, pag. 223-240, Elsevier, Amsterdam

Genin E., Jullien R., Perez F., Fuzeau-Braesch S. 1986. Cuticular hydrocarbons of gregarious and solitary locusts Locusta migratoria cinerascens. J. Chem. Ecol. 12: 1213-1238.

Goncalves de Lima V.M.Q., Roitman I., Kilgour V. 1979. Five Trypanosomatid Species of Insects Distinguished by Isoenzymes. J. Euk. Microbiol. 26 (4): 648-652.

Gorla D.E., Jurberg J., Catalá S.S., Schofield C.J. 1993. Systematics of Triatoma sordida, T. guasayana and T. patagonica (Hemiptera, Reduviidae). Mem. Inst. Oswaldo Cruz 88(3): 379385.

Gorla D.E., Dujardin J.P., Schofield C.J. 1997. Biosystematics of Old World Triatominae. Acta Trop. 63(2): 127-140. 
Gülz P.G. 1994. Epicuticular waxes in the evolution of the plant kingdom. J. Plant Physiol. 143: 453-464.

Hadley N.F. 1978. Cuticular permeability of tenebrionid beetles: correlations with epicuticular hydrocarbon composition. Insect Biochem. 8: 17-22

Hadley N.F., Blomquist G.J., Lanham U.N. 1981. Cuticular hydrocarbons of four species of Colorado Hymenoptera. Insect Biochem. 11: 173-177.

Haas H.L., Tolley K.A. 1998. Geographic variation of wing morphology in three Eurasian populations of the fruit fly, Drosophila lummei. J. Zoology 245: 197-203.

Haverty M.I., Page M., Nelson L.J., Blomquist G.J. 1988. Cuticular hydrocarbons of the dampwood termites, Zootermopsis: Intra- and intercolony variation and potential as taxonomic characters. J. Chem. Ecol. 14: 1035-1058.

Haverty M.I., Nelson L.J., Page M. 1990a. Cuticular hydrocarbons of four populations of Coptotermes formosanus Shiraki in the United States: Similarities and origins of introductions. J. Chem. Ecol. 16: 1635-1647.

Haverty M.I., Thorne B.L., Page M. 1990b. Surface hydrocarbons components of two species of Nasutitermes from Trinidad. J. Chem. Ecol. 16: 2441-2450.

Hoppe K.L., Dillwith J.W., Wright R.E., Szumlas D.E. 1990. Identification of Horse Flies (Diptera: Tabanidae) by Analysis of Cuticular Hydrocarbons. J. Med. Entomol. 27(4): 480486.

Hooper G.H.S., Brown W.V., Lacey M.J., Hunter D. 1996. Cuticular hydrocarbons of the Australian plague locust, Chorticetes terminifera (Walker)(Orthoptera: Acrididae), collected from widely separated geographical locations. Aust. J. Entomol. 35: 257-262. 
Hornos S., Cestau R., Canale D., Diotaiuti L., Pérez R., Panzera F. 1996. Cytogenetic studies of Triatoma guasayana, T. sordida and T. patagonica (Hemiptera: Reduviidae). Mem. Inst. Oswaldo Cruz 91: 123

Howard R.W., McDaniel C.A., Blomquist G.J. 1978. Cuticular hydrocarbons of the Eastern subterranean termite Recticulitermes flavipes (Kollar) (Isoptera: Rhinotermitidae). J. Chem. Ecol. 4: 233-245.

Howard R.W., Blomquist G.J. 1982. Chemical ecology and biochemistry of insect hydrocarbons. A. Rev. Entomol. 27: 149-172.

Huss V.A.R., Ciniglia C., Cennamo P., Cozzolino S., Pinto G., Pollio A. 2002. Phylogenetic relationships and taxonomic position of Chlorella-like isolates from low $\mathrm{pH}$ environments ( $\mathrm{pH}<3.0)$. BMC Evol. Biol. 2: 13-

Hypsa V., Tietz D., Zrzavy J., Rego R.O., Galvão C., Jurberg J. 2002. Phylogeny and biogeography of Triatominae (Hemiptera, Reduviidae): molecular evidence of a New World origin of the asiatic clade. Mol. Phyl. Evol. 23: 447-457.

Jaaska V. 1994. Isoenzyme evidence on the systematics of Hordeum section Marina (Poaceae). Plant. Sys. Evol. 191: 213-226.

Jackson L.L. 1972. Cuticular lipids of insects: IV. Hydrocarbons of the cockroaches Periplaneta japonica and Periplaneta americana compared to other cockroach hydrocarbons. Comp. Biochem. Physiol. 41B: 331-336.

Jackson L.L., Blomquist G.J. 1976. Cuticular lipids of insects: VIII. Alkanes of the mormon cricket Anabrus simplex. Lipids 11: 77-79.

Jackson L.L. 1981. Cuticular lipids of insects - IX. Surface lipids of the grasshoppers Melanoplus bivittatus, Melanoplus femurrubrum and Melanoplus dawsoni. Comp. Biochem. Physiol. 70B: 441-445. 
Jackson L.L. 1982. Cuticular lipids of insects. X. Normal and branched alkanes from the surface of the grasshopper Schistocerca americana. Comp. Biochem. Physiol. 71B: 739-742.

Jackson L.L. 1983. Epicuticular lipid composition of the sand cockroach Arenivaga investigata. Comp. Biochem. Physiol. 74B: 255-257.

Jackson L.L., Bartelt R.J. 1986. Cuticular hydrocarbons of Drosophila virilis: comparison by age and sex. Insect Biochem. 16: 433-439.

Jacob J., Hanssen H.P. 1979. The chemical composition of cuticular lipids from dragonflies (Odonata). Z. Naturforsch. 34C: 498-502.

Jallon J-M., David J.R. 1987. Variations in cuticular hydrocarbons among the eight species of the Drosophila melanogaster subgroup. Evolution 41: 294-302.

Jana S.C. 1994. Isoenzyme profiles in Azotobacteraceae. J. Basic Microbiol. 34(1): 31-35.

Jorgensen J.L., Stehlik I., Brochmann C., Conti E. 2003. Implications of ITS sequences and RAPD markers for the taxonomy and biogeography of the Oxytropis campestris and $O$. arctica (Fabaceae) complexes in Alaska. Am. J. Bot. 90:1470-1480.

Juárez M.P., Brenner R.R., Gros E.G. 1984. The epicuticular lipids of Triatoma infestans. I. Glycerides. Comp. Biochem. Physiol. 78B (2): 427-431.

Juárez M.P., Brenner R.R., 1985. The epicuticular lipids of Triatoma infestans, II. Hydrocarbon dynamics. Comp. Biochem. Physiol. B 82: 793-803.

Juárez M.P., Brenner R.R., Labayén I.L., Gros E.G. 1985. Bioquímica del ciclo evolutivo de Triatoma infestans. VII. Composición de los lípidos de la superficie de la cutícula extraibles con hexano. Acta Physiol. Pharmacol. Latinoam. 35: 223-236.

Juárez M.P., Brenner R.R. 1986. Bioquímica del ciclo evolutivo de Triatoma infestans. IX. Composición de los hidrocarburos cuticulares comparada con otros triatominos. Acta Physiol. Pharmacol. Latinoam. 36: 47-57. 
Juárez M.P., Brenner R.R. 1987. Hydrocarbons of Triatoma pallidipennis. Comp. Biochem. Physiol. 87B (2): 233-239.

Juárez M.P., Brenner R.R. 1989. Fatty acid biosynthesis in the integument tissue of Triatoma infestans. Comp. Biochem. Physiol. B 93: 763-772.

Juárez M.P., Chase J., Blomquist G.J. 1992. A microsomal fatty acid synthetase from the integument of Blatella germanica synthesizes methylbranched fatty acids, precursors to hydrocarbon and contact sex pheromone. Arch. Biochem. Biophys. 293: 333-341.

Juárez M.P., Blomquist G.J. 1993. Cuticular hydrocarbons of Triatoma infestans and Triatoma mazzottii. Comp. Biochem. Physiol. 106B: 667-674.

Juárez M.P. 1994a. Inhibition of cuticular lipid synthesis and its effect on insect survival. Arch. Insect Biochem. Physiol. 25: 177-191.

Juárez M.P. 1994b. Hydrocarbon biosynthesis in Triatoma infestans eggs. Arch. Insect Biochem. Physiol. 25: 193-206.

Juárez, M.P., 1995. The effect of sublethal doses of insecticides on Triatoma infestans lipid synthesis. Pest. Biochem. Physiol. 52, 81-89.

Juárez M.P., Ayala S., Brenner R.R. 1996. Methyl-branched fatty acid biosynthesis in Triatoma infestans. Insect Biochem. Molec. Biol. 26: 593-598.

Juárez M.P., Fernández R., Schofield C.J., Dujardin J.P. 2000. Intergeneric comparison of epicuticular hydrocarbons in Triatominae. Res. Rev. Parasitol. 60: 121-127.

Juárez M.P., Blomquist G.J., Schofield C.J. 2001. Hydrocarbons of Rhodnius prolixus, a Chagas disease vector. Comp. Biochem. Physiol. 129B: 733-746.

Juárez, M.P., Carlson D.A., Salazar-Schettino P.M., Mijailovsky S., Rojas G.. 2002. Cuticular hydrocarbons of Chagas disease vectors in Mexico. Mem. Inst. Oswaldo Cruz 97: 819-827. 
Juárez M.P., Calderón Fernández G. 2007. Cuticular hydrocarbons of triatomines (review). Comp. Biochem. Physiol. 147(3)A: 711-730.

Jurberg J., Galvão C., Lent H., Monteiro F., Macedo Lopes C., Panzera F., Pérez R. 1998. Revalidação de Triatoma garciabesi Carcavallo, Cichero, Martínez, Prosen \& Ronderos, 1967 (Hemiptera: Reduviidae). Entomologia y Vectores 5(3): 107-122.

Jurberg J. 2003. Ferramentas usadas em taxonomia de triatomíneos o uso múltiplo. Entomol. Vect. 10(4): 497-509.

Jurenka R.A., Subchev M. 2000. Identification of Cuticular Hydrocarbons and the Alkene Precursor to the Pheromone in Hemolymph of the Female Gypsy Moth, Lymantria dispar. Arch. Insect Biochem. Physiol. 43: 108-115.

Kaib M., Brandl R., Bagine R.K.N. 1991. Cuticular hydrocarbon profiles: a valuable tool in termite taxonomy. Naturwissenschaften 78: 176-179.

Kaib M., Franke S., Francke W., Brandl R. 2002. Cuticular hydrocarbons in a termite: phenotypes and neighbour-stranger effect. Phys. Entomol. 27: 189-198.

Kaib M., Jmhasly P., Wilfert L., Durka W., Franke S., Francke W., Leuthold R.H., Brandl R. 2004. Cuticular Hydrocarbons and Aggression in the Termite Macrotermes subhyalinus. J. Chem. Ecol. 30 (2): 365-385.

Kolattukudy P.E., Croteau R., Bruckner J.S. 1976. Biochemistry of plant waxes. En: Chemistry and Biochemistry of Natural Waxes, editado por Kolattukudy P.E., pp. 201-233, Elsevier, Amsterdam.

Kovats E. 1965. Gas chromatographic characterization of organic substances in the retention index system. Adv. Chromatogr. 1: 229-247.

Lachenbruch P., Mickey A. 1968. Estimation of error rates in discriminant analysis. Technometrics 10:1-11. 
Leclerc M.C., Guillot J., Deville M. 2000. Taxonomic and phylogenetic analysis of Saprolegniaceae (Oomycetes) inferred from LSU rDNA and ITS sequence comparisons. Antonie van Leeuwenhoek 77(4): 369-377.

Lehmann P., Ordoñez R., Ojeda-Baranda R., Mendez de Lira J., Hidalgo-Sosa L., Monroy C., Ramsey J.M. 2005. Morphometric analysis of Triatoma dimidiata populations (Reduviidae:Triatominae) from Mexico and Northern Guatemala. Mem. Inst. Oswaldo Cruz 100(5): 477-482.

Lemieux B. 1996. Molecular genetics of epicuticular waxes biosynthesis. Trends Plant Sci. 1, $312-318$.

Lent H., Wygodzinsky P. 1979. Revision of the Triatominae (Hemiptera, Reduviidae), and their significance as vector of Chagas' disease. Bull. Am. Mus. Nat. Hist. 163: 123-520.

Lent H., Jurberg J. 1985. Sobre a variaçâo intra-especifica em Triatoma dimidiata (Latreille) e Triatoma infestans (Klug) (Hemiptera: Reduviidae). Mem. Inst. Oswaldo Cruz 80: 285-299.

Locke M. 1965. Permeability of the insect cuticle to water and lipids. Science 147: 295-298.

Lockey K.H. 1976. Cuticular hydrocarbons of Locusta, Schistocerca and Periplaneta and their role in waterproofing. Insect Biochem. 6: 457-472.

Lockey K.H. 1978. The adult cuticular hydrocarbons of Tenebrio molitor L. and Tenebrio obscurus F. (Coleoptera: Tenebrionidae). Insect Biochem. 8: 237-250.

Lockey K.H. 1980. Insect cuticular hydrocarbons. Comp. Biochem. Physiol. 65B: 457-462.

Lockey K.H. 1985a. Insect cuticular lipids. Comp. Biochem. Physiol. 81B: 263-273.

Lockey K.H. 1985b. cuticular hydrocarbons of adult Eurychora sp. (Coleoptera: Tenebrionidae). Comp. Biochem. Physiol. 81B: 223-227. 
Lockey K.H. 1988. Lipids of the insect cuticle: origin, composition and function. Comp. Biochem. Physiol. 89B (4): 595-645.

Lockey K.H. 1991. Insect hydrocarbon classes: Implications for chemotaxonomy. Insect Biochem. 21: 91-97.

Lukashevich E.D., Mostovski M.B. 2003. Hematophagous insects in the fossil record. Paleontol. J. 37: 153-161.

Lyman D.E., Monteiro F.A., Escalante A.A., Cordon-Rosales C., Wesson D.M., Dujardin J.P., Beard C.B. 1999. Mitochondrial DNA sequence variation among triatomine vectors of Chagas' disease. Am. J. Trop. Med. Hyg., 60(3): 377-386.

Maffei M. 1996. Chemotaxonomic significance of leaf wax alkanes in the Gramineae. Biochem. Syst. Ecol. 24: 53-64.

Mamuris Z., Stamatis C., Bani M., Triantaphyllidis C. 1999. Taxonomic relationships between four species of the Mullidae family revealed by three genetic methods: allozymes, random amplified polymorpic DNA and mitochondrial DNA. J. Fish Biol. 55(3): 572-587.

Marcilla A., Bargues M.D., Ramsey J.M., Magallon-Gastelum E., Salazar-Schettino P.M., Abad-Franch F., Dujardin J.P., Schofield C.J., Mas-Coma S. 2001. The ITS-2 of the nuclear rDNA as a molecular marker for populations, species, and phylogenetic relationships in Triatominae (Hemiptera: Reduviidae), vectors of Chagas disease. Mol. Phylogenet. Evol. 18(1): 136-142.

Marcilla A., Bargues M.D., Abad-Franch F., Panzera F., Carcavallo R.U., Noireau F., Galvao C., Jurberg J., Miles M.A., Dujardin J.P., Mas-Coma S. 2002. Nuclear rDNA ITS-2 sequences reveal polyphyly of Panstrongylus species (Hemiptera: Reduviidae: Triatominae), vectors of Trypanosoma cruzi. Inf. Gen. Evol. 1(3): 225-235.

Martínez F.H., Villalobos G.C., Cevallos A.M., Torre P. de L., Laclette J.P., AlejandreAguilar R., Espinoza B. 2006. Taxonomic study of the phyllosoma complex and other triatomine (Insecta: Hemiptera: Reduviidae) species of epidemiological importance in the 
transmission of Chagas disease: using ITS-2 and mtCytB sequences. Mol. Phyl.. Evol. 41(2): 279-287.

Mayr E. 1970. Populations, Species, and Evolution. Harvard Univ. Press. Cambridge, Mass.

McCarthy E.D., Han J., Calvin M. 1968. Hydrogen Atom Transfer in Mass Spectrometric Fragmentation Patterns of Saturated Aliphatic Hydrocarbons. Anal. Chem. 40(10): 14751480 .

Medina E., Aguiar G., Gómez A., Aranda J., Medina J.D., Winter K. 2006. Taxonomic significance of the epicuticular wax composition in species of the genus Clusia from Panama. Biochem. Syst. Ecol. 34: 319-326.

Milligan P.J.M., Phillips A, Molyneux D.H. 1986. Differentiation of Anopheles culicifacies Giles (Diptera: Culicidae) sibling species by analysis of cuticular components. Bull. Ent. Res. 76: 529-537.

Mimura M.R., Salatino M.L.F., Salatino A., Baumgratz J.F.A. 1998. Alkanes from foliar epicuticular waxes of Huberia species: taxonomic implications. Biochem. Syst. Ecol. 26: 581588.

Monroy M.C., Bustamante D.M., Rodas A.G., Enriquez M.E., Rosales R.G. 2003a. Habitats, Dispersion and Invasion of Sylvatic Triatoma dimidiata (Hemiptera:Reduviidae:Triatominae) in Peten, Guatemala. J. Med. Entomol. 40(6): 800-806.

Monroy C., Rodas A., Mejia M., Rosales R., Tabaru Y. 2003b. Epidemiology of Chagas disease in Guatemala: infection rate of Triatoma dimidiata, Triatoma nitida and Rhodnius prolixus (Hemiptera, Reduviidae) with Trypanosoma cruzi and Trypanosoma rangeli (Kinetoplastida, Trypanosomatidae). Mem. Inst. Oswaldo Cruz. 98: 305-310.

Monteiro F.A., Pérez R., Panzera F., Dujardin J.P., Galvão C., Rocha D., Noireau F., Schofield C.J., Beard C.B. 1999. Mitochondrial DNA variation of Triatoma infestans populations and its implication on the specific status of T. melanosoma. Mem. Inst. Oswaldo Cruz. 94(1): 229-238. 
Monteiro F.A., Donnelly M.J., Beard C.B., Costa J. 2004. Nested clade and phylogeographic analyses of the Chagas disease vector Triatoma brasiliensis in Northeast Brazil. Mol. Phyl. Evol. 32: 46-56.

Morel C.M., Lazdins J. 2003. Chagas disease. Nature Rev. Microbiol. 1: 14-15.

Nakagawa J., Cordon-Rosales C., Juárez J., Nonami T. 2003a. Impact of residual spraying on Rhodnius prolixus and Triatoma dimidiata in the department of Zacapa in Guatemala. Mem. Inst. Oswaldo Cruz. 98: 277-281.

Nakagawa J., Hashimoto K., Cordon-Rosales C., Juárez J., Trampe R., Marroquin L. 2003 b. The impact of vector control on Triatoma dimidiata in the Guatemalan department of Jutiapa. Ann. Trop. Med. Parasitol. 97: 288-297.

Napolitano R., Juárez M.P. 1997. Entomopathogenous fungi degrade epicuticular hydrocarbons of Triatoma infestans. Arch. Biochem. Biophys. 344: 208-214.

Nelson D.R., Sukkestad D.R. 1975. Normal and branched alkanes from the cast skins of the grasshopper Schistocerca vaga (Scudder). J. Lipid Res. 16: 12-18.

Nelson D.R., Dillwith J.W., Blomquist G.J. 1981. Cuticular hydrocarbons of the housefly Musca domestica. Insect Biochem. 11: 187-197.

Nelson D.R., Nunn N.N., Jackson L.L. 1984. Re-analysis of the methylalkanes of the grasshoppers Melanoplus differentialis, M. packardii and M. sanguinipes. Insect Biochem. 14: 677-683.

Nelson D.R., Carlson D.A. 1986. Cuticular hydrocarbons of the tse-tse flies Glossina morsitans morsitans, G. austeni and G. pallidipes. Insect Biochem. 16: 403-416.

Nelson D.R., Carlson D.A., Fatland C.L. 1988. Cuticular hydrocarbons of tsetse flies II: Glossina fuscipes fuscipes, G. palpalis palpalis, G. p. gambiensis, G. tachinoides, and G. brevipalpis. J. Chem. Ecol. 14: 963-987. 
Nelson D.R., Fatland C., Adams T. 2002. Methyl-Branched Alkanes on the Surface of Male and Female Adults, and Eggs of the Colorado Potato Beetle, Leptinotarsa decemlineata. Proceedings of the Molecular Insect Science International Symposium.

Noireau F., Gutierrez T., Zegarra M., Flores R., Brenière F., Cardozo L., Dujardin J.P. 1998. Cryptic speciation in Triatoma sordida (Hemiptera: Reduviidae) from the Bolivian Chaco. Trop. Med. Int. Health 3(5): 364-372.

Noireau F., Zegarra M., Ordoñez J., Gutierrez T., Dujardin J.P. 1999a. Genetic Structure of Triatoma sordida (Hemiptera: Reduviidae) Domestic Populations from Bolivia: Application on Control Interventions. Mem. Inst. Oswaldo Cruz 94(3): 347-351.

Noireau F., Gutierrez T., Flores R., Brenière F., Bosseno M.F., Wisnivesky-Colli. 1999b. Ecogenetics of Triatoma sordida and Triatoma guasayana (Hemiptera: Reduviidae) in the Bolivian Chaco. Mem. Inst. Oswaldo Cruz 94(4): 451-457.

Oscherov E.B., Damborsky M.P., Bar M.E. 1998. Características biológicas de Triatoma sordida (Heteroptera: Reduviidae): Ciclo de vida. Rev. Soc. Entomol. Argent. 57: 13-17.

Ostman C. 1982. Isoenzymes and Taxonomy in Scandinavian Hydroids (Cnidaria, Campanulariidae). Zool. Scripta 11(3): 155-163.

Pacak A., Fiedorow P., Dabert J., Szweykowska-kulińska Z. 1998. RAPD technique for taxonomic studies of Pellia epiphylla complex (Hepaticae, Metzgeriales). Genetica 104(2): 179-187.

Page M., Nelson L.J., Haverty M.I., Blomquist G.J. 1990a. Cuticular hydrocarbons of eight species of North American cone beetles, Conophthorus Hopkins. J. Chem. Ecol. 16: 11731198.

Page M., Nelson L.J., Haverty M.I., Blomquist G.J. 1990b. Cuticular hydrocarbons as chemotaxonomic characters for bark beetles: Dendroctonus ponderosae, D. jeffreyi, D. brevicomis and D. frontalis (Coleoptera: Scolytidae). Ann. Entomol. Soc. Am. 83: 892-901. 
Page M., Nelson L.J., Blomquist G.J., Seybold S.J. 1997. Cuticular Hydrocarbons as Chemotaxonomic Characters of Pine Engraver Beetles (Ips spp.) in the grandicollis Subgeneric Group. J. Chem. Ecol. 23(4): 1053-1099.

Panzera F., Hornos S., Pereira J., Cestau R., Canale D.M., Diotaiuti L., Dujardin J.P., Perez R. 1997. Genetic variability and geographic differentiation among three species of triatomine bugs (Hemiptera: Reduviidae). Am. J. Trop. Med. Hyg. 57(6): 732-739.

Panzera F., Ferrandis I., Ramsey J., Ordonez R., Salazar-Schettino P.M., Cabrera M., Monroy M.C., Bargues M.D., Mas-Coma S., O’Connor J.E., Angulo V.M., Jaramillo N., CordonRosales C., Gomez D., Perez R. 2006. Chromosomal variation and genome size support existence of cryptic species of Triatoma dimidiata with different epidemiological importance as Chagas disease vectors. Trop. Med. Int. Health 11: 1092-1103.

Pastorini J., Thalmann U., Martin R.D. 2003. A Molecular Approach to Comparative Phylogeography of Extant Malagasy Lemurs. PNAS 100(10): 5879-5884.

Patterson J.S., Schofield C.J., Dujardin J.P., Miles M.A. 2001. Population morphometric analysis of the tropicopolitan bug Triatoma rubrofasciata and relationships with old world species of Triatoma: evidence of New World ancestry. Med. Vet. Entomol. 15(4): 443-451.

Paul B. 2000. ITS1 region of the rDNA of Pythium megacarpum sp. nov., its taxonomy, and its comparison with related species. FEMS Microbiol. Lett. 186(2): 229-233.

Paulin-Mahady A.E., Harrington T.C., McNew D. 2002. Phylogenetic and taxonomic evaluation of Chalara, Chalaropsis, and Thielaviopsis anamorphs associated with Ceratocystis. Mycologia 94(1): 62-72.

Pedrini N. 2006. Enzimas fúngicas involucradas en el catabolismo de hidrocarburos. Su aplicación a la optimización de bioinsecticidas. Tesis de la Facultad de Ciencias Exactas, UNLP. 
Pereira J., Dujardin J.P., Salvatella R., Tibayrenc M. 1996. Enzymatic variability and phylogenetic relatedness among Triatoma infestans, $T$. platensis, $T$. delpontei and $T$. rubrovaria. Heredity $77:$ 47-54.

Petana W.B. 1971. American trypanosomiasis in British Honduras. X: natural habitats and ecology of Triatoma dimidiata (Hemiptera, Reduviidae) in the El Cayo and Toledo districts, and the prevalence of infection with Trypanosoma (Schizotrypanum) cruzi in the wild-caught bugs. Ann. Trop. Med. Parasitol. 65: 169-178.

Petersen G.H., Russell P.J.C. 1971. Cardium hauniense compared with C. exiguum and $C$. glaucum. J. Mollus. Stud. 39: 409-420.

Phillips A., Walsh J.F., Garms R., Molyneux D.H., Milligan P., Ibrahim G. 1985. Identification of adults of the Simulium damnosum complex using hydrocarbon analysis. Trop. Med. Parasitol. 36: 97-101.

Picollo M.I., Vassena C.V., Santo Orihuela P., Barrios S., Zaidemberg M., Zerba E.N. 2005. High resistance to pyrethroid insecticides associated with ineffective field treatments in Triatoma infestans (Hemiptera: Reduviidae) from Northern Argentina. J. Med. Entomol. 42: 637-642.

Pietrokovsky S., Bottazzi V., Gajate P., Canale D.M., Wisnivesky-Colli C. 1994. Studies on reproductive compatibility between Triatoma sordida and Triatoma guasayana. Mem. Inst. Oswaldo Cruz 89 (Suppl. I): 379-385.

Pimentel R.A. 1992. An Introduction to Ordination, Principal Components Analysis and Discriminant Analysis. En: Ordination In The Study of Morphology, Evolution and Systematics of Insects: Applications and Quantitative Genetic Rationals; editado por Sorensen J.T. y Foottit R.G., $1^{\circ}$ edición, pag. 11-28, Elsevier, Amsterdam

Proctor M.C.F., Proctor M.E., Groenhof A.C. 1989. Evidence from Peroxidase Polymorphism on the Taxonomy and Reproduction of Some Sorbus Populations in South-West England. New Phytol. 112: 569-575. 
Ramírez C.J., Jaramillo C.A., Delgado M.P., Pinto N.A., Aguilera G., Guhl F. 2005. Genetic structure of sylvatic, peridomestic and domestic populations of Triatoma dimidiata (Hemiptera: Reduviidae) from an endemic zone of Boyaca, Colombia. Acta Trop. 93: 23-29.

Ramos A.M., Forchiassin F., Ranalli M.E. 1999. Uso de isoenzimas de endo-ß-d-1,4glucanasa y variables fisiológicas para la delimitación de especies del género Saccobolus. Polibotanica 10: 23-38.

Rebagliati P., Papeschi A.G., Mola L.M., Pietrokovsky S., Gajate P., Bottazzi V., Wisnivesky-Colli C. 1998. Comparative Meiotic Studies in Triatoma sordida (Stal) and T. guasayana Wygodzinsky \& Abalos (Reduviidae, Heteroptera). Mem. Inst. Oswaldo Cruz 93(3): 309-315.

Reed J.R., Vanderwel D., Choi S., Pomonis J.G., Reitz R.C., Blomquist G.J. 1994. Unusual mechanism of hydrocarbon formation in the housefly: cytochrome P450 converts aldehyde to the sex pheromone component (Z)-9-tricosene and $\mathrm{CO}_{2}$. Proc. Natl. Acad. Sci. USA 91(21): $10000-10004$.

Rioux J.A., Lanotte G., Serres E., Pratlong F., Bastien P., Perieres J. 1990. Taxonomy of Leishmania. Use of isoenzymes. Suggestions for a new classification. Ann. Parasitol. Hum. Comp. 65(3):111-125.

Rohlf J. 1990. Morphometrics. Annu. Rev. Ecol. Syst. 21: 299-316.

Sainz A.C., Mauro L.V., Moriyama E.N., García B.A. 2004. Phylogeny of triatomine vectors of Trypanosoma cruzi suggested by mitochondrial DNA sequences. Genetica 121: 229-240.

Schneider-Belhaddad F., Kolattukudy P. 2000. Solubilization, partial purification, and characterization of a fatty aldehyde decarbonylase from a higher plant, Pisum sativum. Arch. Biochem. Biophys. 377: 341-349.

Schofield C.J. 2002. Evolución y control de Triatoma dimidiata. En: Taller para el establecimiento de pautas técnicas en el control de Triatoma dimidiata. PAHO document OPS/HCP/HCT/214/02, San Salvador, PAHO, pp. 12-18. 
Schofield C.J., Jannin J., Salvatella R. 2006. The future of Chagas disease control. Trends Parasitol. 12:583-588.

Shipunov A.B., Bateman R.M. 2005. Geometric morphometrics as a tool for understanding Dactylorhiza (Orchidaceae) diversity in European Russia. Biol. J. Linn. Soc. 85(1): 1-12.

Sing S., Brar J.K., Sandhu D.K., Kaur A. 1996. Isozyme polymorphism of cellulases in Aspergillus terreus. J. Basic Microbiol. 36: 289-296.

Sokal R. R. 1973. The species problem reconsidered. Sys. Zool. 22: 360-374.

Sokal R.R., Rohlf F.J. 2001. Biometry $3^{\circ}$ ed. W. H. Freeman and Company, New York.

Soliday C.L., Blomquist G.J., Jackson L.L. 1974. Cuticular lipids of insects - IV Cuticular lipids of the grasshoppers, Melanoplus sanguinipes and Melanoplus packardii. J. Lipid Res. 15: 339-405.

Sonnenberg R., Nolte A.W., Tautz D. 2007. An evaluation of LSU rDNA D1-D2 sequences for their use in species identification. Frontiers Zool. 4: 6-

Sponsler R.C., Apel A.G. 1990. Aspects of the water relations of the Formosan and Eastern subterranean termites (Isoptera: Rhinotermitidae). Environ. Entomol. 19: 15-20.

Tartarotti E., Ceron C.R. 2005. Ribosomal DNA ITS-1 Intergenic Spacer Polymorphism in Triatomines (Triatominae, Heteroptera). Biochem. Gen. 43: 365-373.

Tibayrenc M., Miles M.A. 1983. A genetic comparison between Brazilian and Bolivian zymodemes of Trypanosoma cruzi. Trans. R. Soc. Trop. Med.Hyg. 77(1): 76-83.

Usinger R. 1944. The Triatominae of North and Central America and the West Indies and their public health significance. Pub. Health Bull. 288 
Usinger R.L., Wygodzinsky P., Ryckman R.E. 1966. The biosystematics of Triatominae. Annu. Rev. Entomol. 11: 309-330.

Verdyck P. 1999. Biochemical Systematics of the Phyllotreta cruciferae Complex (Coleoptera: Chrysomelidae: Alticinae). Ann. Entomol. Soc. Am. 92(1): 30-39.

Vinhaes M.C., Dias J.C.P. 2000. Doença de Chagas no Brasil. Cad. Saúde Publ. 16: 7-12.

Warthen J.D., Uebel E.C. 1980. Comparison of the unsaturated cuticular hydrocarbons of the male and female house crickets, Acheta domesticus (L.) (Orthoptera: Gryllidae). Insect Biochem. 10: 435-439.

Warthen J.D., Uebel E.C., Lusby W.R., Adler V.E. 1981. The cuticular lipids of the walking stick Diapheromera femorata (Say). Insect Biochem. 11: 467-472.

Wigglesworth V.B. 1945. Transpiration through the cuticle of insects. J. Exp. Biol. 21: 97 114.

Wilks S.S. 1932. Certain generalizations in the analysis of variance. Biometrika 24: 471.

Woodrow R.J., Grace J.K., Nelson L.J., Haverty M.I. 2000. Modification of Cuticular Hydrocarbons of Cryptotermes brevis (Isoptera: Kalotermitidae) in Response to Temperature and Relative Humidity. Envirom. Entomol. 29(6): 1100-1107.

Zaccara S., Stefanib F., Gallic P., Nardid P.A., Crosa G. 2004. Taxonomic implications in conservation management of white-clawed crayfish (Austropotamobius pallipes) (Decapoda, Astacidae) in Northern Italy. Biol. Cons. 120(1): 1-10.

Zeledón R., Solano G., Zuniga A., Swartzwelder J.C. 1973. Biology and ethology of Triatoma dimidiata (Latreille, 1811). 3: habitat and blood sources. J. Med. Entomol. 10: 363-370.

Zeledón R. 1981. El Triatoma dimidiata (Latreille, 1811) y su relación con la enfermedad de Chagas. EUNED, San José, Costa Rica. 164 pp. 
Zeledón R. 1983. Vectores de la enfermedad de Chagas y sus caracteristicas ecofisiológicas. Interciencia 8 (6): 384-395.

Zeledón R., Ugalde J.A., Paniagua L.A. 2001. Entomological and ecological aspects of six sylvatic species of Triatomines (Hemiptera, Reduviidae) from the Collection of the National Biodiversity Institute of Costa Rica, Central America. Mem. Inst. Oswaldo Cruz. 96: 757764. 


\section{Abreviaturas}

Aquí se presenta una lista de las abreviaturas más usadas.

ANOVA: análisis de la varianza

ANCOVA: análisis de la covarianza

CGC: cromatografía gaseosa capilar

CGC-MS: cromatografía gaseosa capilar acoplada a espectrometría de masa

DA: análisis discriminante

Es o et: error estándar o típico, de la media.

FD o DF: función discriminante.

FID: detector de ionización de llama.

HC: hidrocarburo o hidrocarburos, en su caso.

ITS: espaciador interno de transcripción del DNA ribosómico

KI: índice de Kovats

$\mathrm{M}^{+}$: ión molecular

MS: espectrometría de masa

PCA: análisis de componentes principales.

rDNA: ácido desoxirribonucléico que codifica para ribosomas

RAPD: amplificación aleatoria de fragmentos polimórficos de DNA.

Tg x Ts: insectos híbridos resultado de cruzar ejemplares de T. guasayana con T. sordida.

UPGMA: agrupamiento de a pares no ponderado basado en la media aritmética de los coeficientes de similitud de los pares en unión. 